Mixture Models Versus Free for Waste Glass Durability

Greg Piepel

Trish Redgate

Paul Masuga

March 1996

Prepared for the U.S. Department of Energy under Contract DE-AC06-76RLO 1830

Pacific Northwest Laboratory

Operated for the U.S. Department of Energy by Battelle Memorial Institute 
This report was prepared as an account of work sponsored by an agency of the United States Government. Neither the United States Government nor any agency thereof, nor Battelle Memorial Institute, nor any of their employees, makes any warranty, express or implied, or assumes any, legal liability or responsibility for the accuracy, completeness., or usefulness of any information, apparatus, product, or process disclosed, or represents that its use would not infringe privately owned rights. Reference herein to any specific commercial product, process, or service by trade name, trademark, manufacturer, or otherwise does not necessarily constitute or imply its endorsement, recommendation, or favoring by the United States Government or any agency thereof, or Battelle Memorial Institute. The views and opinions of authors expressed herein do not necessarily state or reflect those of the United States Government or any agency thereof.

\section{PACIFIC NORTHWEST NATIONAL LABORATORY operated by \\ BATTELLE. \\ for the \\ UNITED STATES DEPARTMENT OF ENERGY under Contract DE-ACO6-76RLO 1830}

Printed in the United States of America

Available to DOE and DOE contractors from the

Office of Scientific and Technical Information, P.O. Box 62, Oak Ridge, TN 37831: prices available from (615) $576-8401$.

Available to the public from the National Technical Information Service, U.S. Department of Commerce, 5285 Port Royal Rd., Springfield, VA 22161 
MIXTURE MODELS VERSUS FREE ENERGY OF HYDRATION MODELS FOR WASTE GLASS DURABILITY

Greg Piepel

Trish Redgate

Paul Masuga

March 1996

Pacific Northwest National Laboratory*

Richland, Washington 99352

* Pacific Northwest National Laboratory is a multiprogram laboratory operated by Battelle Memorial Institute for the U.S. Department of Energy under Contract DE-AC06-76RLO 1830. 



\title{
Mixture Models Versus Free Energy of Hydration Models for Waste Glass Durability
}

\author{
Greg Piepel, Trish Redgate, and Paul Masuga \\ Pacific Northwest National Laboratory(2) \\ P.O. Box 999, Mail Stop K5-12 \\ Richland, Washington 99352
}

\begin{abstract}
Two approaches for modeling high-level waste glass durability as a function of glass composition are compared. The mixture approach utilizes first-order mixture (FOM) or secondorder mixture (SOM) polynomials in composition, whereas the free energy of hydration (FEH) approach assumes durability is linearly related to the FEH of glass. Both approaches fit their models to data using least squares regression.

The mixture and FEH approaches are used to model glass durability as a function of glass composition for several simulated waste glass data sets. The resulting FEH and FOM model coefficients and goodness-of-fit statistics are compared, both within and across data sets. The goodness-of-fit statistics show that the FOM model fits/predicts durability in each data set better (sometimes much better) than the FEH model. Considerable differences also exist between some FEH and FOM model component coefficients for each of the data sets. These differences are due to the mixture approach having a greater flexibility to account for the effect of a glass component depending on the level and range of the component and on the levels of other glass components. The mixture approach can also account for higher-order (e.g., curvilinear or interactive) effects of components, whereas the FEH approach cannot. SOM models were developed for three of the data sets, and are shown to improve on the corresponding FOM models. Thus, the mixture approach has much more flexibility than the FEH approach for approximating the relationship between glass composition and durability for various glass composition regions.
\end{abstract}

(a) Pacific Northwest National Laboratory is a multiprogram laboratory operated by Battelle Memorial Institute for the U.S. Department of Energy under Contract DE-AC06-76RLO 1830. 



\section{Summary}

Two approaches for modeling high-level waste glass durability as a function of glass composition are compared. The mixture approach fits first- or second-order polynomials in composition to durability data using least squares regression. The free energy of hydration (FEH) approach assumes durability is linearly related to the FEH of glass, with the line fitted to data by least squares regression. The FEH of a glass is calculated as a composition-weighted linear combination of free energies of hydration of the glass components. The FEH approach is shown to be a restricted version of the first-order mixture (FOM) approach.

The mixture and FEH approaches are compared in terms of their ability to model Product Consistency Test (PCT) normalized boron releases as a function of glass composition for several simulated waste glass data sets. Least squares regression was used to fit FEH and FOM models to each data set. Goodness-of-fit statistics show that the FOM model fits/predicts PCT boron release in each data set better (sometimes much better) than the FEH model. The model $\mathbf{R}^{2}$ (proportion of variation in PCT boron releases accounted for by a model) statistics are summarized in Table S.1 to illustrate this point.

Considerable differences also exist between some FEH and FOM model component coefficients for each of the data sets. Comparing FOM coefficients across the data sets shows that the effect of a glass component on PCT normalized boron release can depend on the level and range of the component and on the levels of other glass components. The FEH approach has a limited ability to represent such behavior for different glass composition regions, due to its reliance on assumed constant effects of each component. The mixture approach, on the other hand, determines the effects of glass components on durability from a given data set. It can also account for higher-order (e.g., curvilinear or interactive) effects of components. Second-order mixture (SOM) models were developed for three of the data sets, and are shown to improve on the corresponding FOM models (see $\mathrm{R}^{2}$ statistics in Table S.1).

It is concluded that the mixture approach is more flexible than the FEH approach for approximating the relationship between glass composition and durability for various glass composition regions.

Table S.1. $\mathrm{R}^{2}$ Statistics for FEH, FOM, and SOM Models Fitted to Several Data Sets

\begin{tabular}{|c|c|c|c|c|c|c|c|c|c|c|c|c|}
\hline \multirow[b]{2}{*}{ Model } & \multicolumn{12}{|c|}{ Data Set } \\
\hline & $\# 1$ & $\# 2$ & $\# 3$ & $\# 4$ & $\# 5$ & $\# 6$ & \#7 & \#8 & \#9 & $\# 10$ & $\# 11 \mathrm{a}$ & $\# 11 \mathrm{~b}$ \\
\hline FEH & .472 & .129 & .113 & .537 & .741 & .400 & .730 & .326 & .506 & .620 & .763 & .764 \\
\hline FOM & .954 & .794 & .694 & .911 & .899 & .810 & .880 & .917 & .980 & .842 & .961 & .961 \\
\hline SOM & n.a. & n.a. & n.a. & n.a. & n.a. & .892 & .931 & n.a. & n.a. & .923 & n.a. & n.a. \\
\hline
\end{tabular}





\section{Acknowledgments}

Greg Piepel and Trish Redgate began this work in late 1994 and early 1995 as part of the high-level waste glass formulation activity of the Pacific Northwest Laboratory (PNL) Vitrification Technology Development (PVTD) project funded by the U.S. Department of Energy (DOE). A large amount of the work was performed on their personal time (after a PVTD scope and funding cut). Finishing touches on the report were partially funded by the Waste Acceptance Technical Review Group, which is also funded by the DOE. Paul Masuga performed part of the work while at PNL from January to May 1995 as a Student Fellow under the Science and Engineering Research Semester (SERS) program. The SERS program is funded by the U.S. Department of Energy and is administered by the Northwest Division of Associated Western Universities, Inc.

The authors acknowledge Dr. Carol Jantzen and Dr. John Plodinec of the Savannah River Technology Center for providing pre-publication information about the free energy of hydration modeling approach as well as the data set used to develop the free energy of hydration durability models for the Defense Waste Processing Facility at the Savannah River Site.

Also acknowledged are John Vienna and Rick Bates for providing glass science and statistical reviews, respectively, and Frank Ryan for providing the editorial review. All three provided comments that led to improvements in the report. 



\section{Contents}

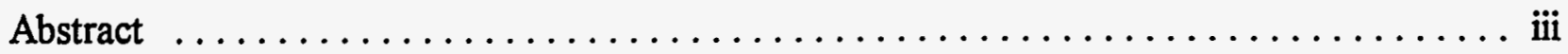

Summary $\ldots \ldots \ldots \ldots \ldots \ldots \ldots \ldots \ldots \ldots \ldots \ldots \ldots \ldots \ldots \ldots \ldots \ldots, v$

Acknowledgments $\ldots \ldots \ldots \ldots \ldots \ldots \ldots \ldots \ldots \ldots \ldots \ldots \ldots \ldots \ldots \ldots \ldots \ldots \ldots \ldots$

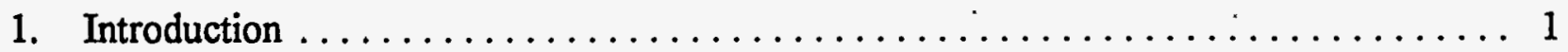

2. Mixture and Free Energy of Hydration Models $\ldots \ldots \ldots \ldots \ldots \ldots \ldots \ldots \ldots$

2.1 Mixture Models..$\ldots \ldots \ldots \ldots \ldots \ldots \ldots \ldots \ldots \ldots \ldots \ldots \ldots$

2.2 Free Energy of Hydration Models $\ldots \ldots \ldots \ldots \ldots \ldots \ldots \ldots \ldots$

2.3 Numbers of Components and Logarithm of Durability $\ldots \ldots \ldots \ldots \ldots \ldots$

3. Comparing Mixture and Free Energy of Hydration Model Forms $\ldots \ldots \ldots \ldots \ldots 7$

3.1 Writing the FEH Model in the Form of a FOM Model $\ldots \ldots \ldots \ldots \ldots \ldots$

3.2 Deriving Coefficients from a FOM Model That Can Be Compared to Component Free Energy of Hydration Values $\ldots \ldots \ldots \ldots \ldots \ldots \ldots \ldots .8$

3.3 A Hybrid FEH/FOM Modeling Approach $\ldots \ldots \ldots \ldots \ldots \ldots \ldots \ldots$

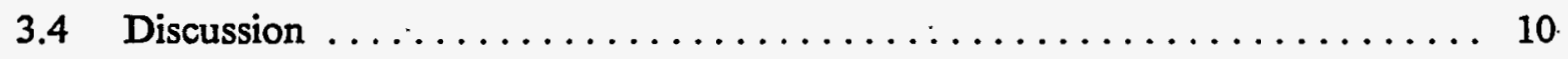

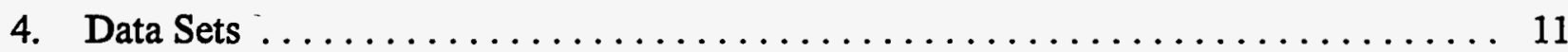

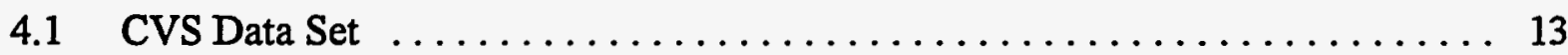

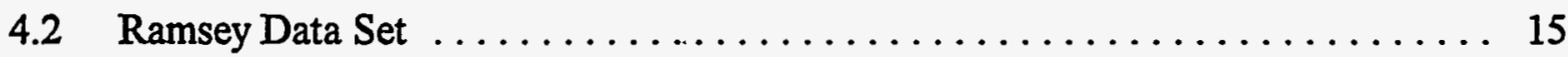

4.3 West Valley 1987 and 1988 Data Sets $\ldots \ldots \ldots \ldots \ldots \ldots \ldots \ldots \ldots \ldots \ldots \ldots \ldots$

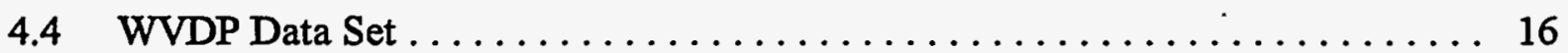

4.5 SRTC/DWPF Homogenous Glasses Data Set $\ldots \ldots \ldots \ldots \ldots \ldots \ldots \ldots$

5. Statistics Used to Compare Models $\ldots \ldots \ldots \ldots \ldots \ldots \ldots \ldots \ldots \ldots \ldots \ldots$

6. Mixture and Free Energy of Hydration Models Fitted to Data Sets ........... 23 


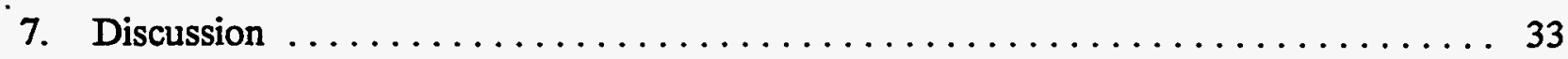

7.1 Free Energy of Hydration Model Results $\ldots \ldots \ldots \ldots \ldots \ldots \ldots \ldots \ldots$

$7.2 \quad$ First-Order Mixture Model Results $\ldots \ldots \ldots \ldots \ldots \ldots \ldots \ldots \ldots \ldots \ldots$

7.3 Second-Order Mixture Model Results $\ldots \ldots \ldots \ldots \ldots \ldots \ldots \ldots \ldots . \ldots \ldots$

7.4 Comparing Goodness-of-Fits of Mixture and Free Energy of Hydration Models . . 35

7.5 Comparing FEH and FOM Model Coefficients $\ldots \ldots \ldots \ldots \ldots \ldots \ldots \ldots$

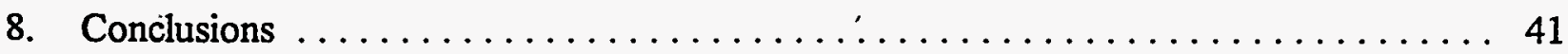

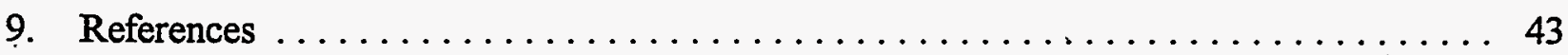

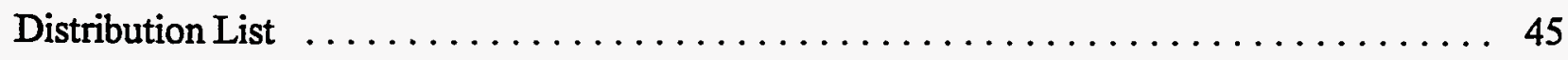

Appendix A. Data Sets-.Glass Compositions and PCT Normalized Releases ......... A.1

Appendix B. Scatterplot Matrices $\ldots \ldots \ldots \ldots \ldots \ldots \ldots \ldots \ldots \ldots \ldots \ldots \ldots \ldots \ldots \ldots$ B.1

Appendix C. Predicted Versus Measured Plots ..................... C.1 


\section{Tables.}

1. Component Free Energy of Hydration Values Used In This Work . . . . . . . . . 5

2. Ranges of Glass Components and PCT Normalized B Release . . . . . . . . . . . 12

3. Coefficients and Goodness-of-Fit Statistics for Free Energy of

Hydration Models Fitted to $\ln \left(\mathrm{PCT}\right.$ Normalized B Release in $\left.\mathrm{g} / \mathrm{m}^{2}\right) \ldots \ldots \ldots$

4. Coefficients, Coefficient Standard Deviations (in parentheses), and Goodness-of-Fit Statistics for First-Order Mixture Models and Rewritten

Free Energy of Hydration Models for $\ln \left(\right.$ PCT Normalized B Release, $\mathrm{g} / \mathrm{m}^{2}$ ) . . . . . . 26

5. Coefficients of Others Components for Free Energy of Hydration

Models Written in the Form of First-Order Mixture Models for

In(PCT Normalized B Release in $\mathrm{g} / \mathrm{m}^{2}$ ) .

6. Coefficients, Coefficient Standard Deviations (in parentheses), and

Goodness-of-Fit Statistics for Reduced Second-Order Mixture

Models for $\ln$ (PCT Normalized B Release, $\mathrm{g} / \mathrm{m}^{2}$ ) 



\section{Introduction}

Nuclear waste glasses must satisfy several property constraints in order to be processable in a melter and acceptable for eventual disposal in a geologic repository. While these properties can be experimentally measured for any glass composition of interest, it is desirable to develop models. that predict glass properties as functions of glass composition. If a model that adequately approximates the true relationship between a glass property and glass composition can be developed, property values can then be predicted for any composition within the model's domain (the composition region where the model is applicable). Predictive glass property models are very useful in formulating glass compositions with desired properties, and can be used in operating and controlling a nuclear waste vitrification plant.

A very important waste glass property is chemical durability (resistance to dissolution in water). The normalized elemental release of boron (B) from a 7-day Product Consistency Test $(\mathrm{PCT})^{1}$ is used as a measure glass durability in this report. Using several data sets, the performance of two approaches for modeling and predicting the PCT B release of simulated highlevel nuclear waste glasses as a function of glass composition are compared. The mixture and free energy of hydration (FEH) modeling approaches are described in Section 2. There are many options for mixture models ${ }^{2}$, but attention is restricted in this report to Scheffé first-order mixture (FOM) and second-order mixture (SOM) models, as described in Section 2. Section 3 compares the mathematical forms of the FEH and FOM models, and describes how to compare their coefficients. The data sets used to compare the fits and coefficients of FEH and mixture (FOM and SOM) models are discussed in Section 4. Section 5 describes the goodness-of-fit statistics used to assess how well the FEH and mixture models fit/predict the various data sets. Section 6 presents the results of fitting FEH and FOM models to each of the data sets, and SOM models to three of the data sets. The results are discussed in Section 7. Conclusions are given in Section 8. References are listed in Section 9. 



\section{Mixture and Free Energy of Hydration Models}

The mathematical forms of first-order and second-order mixture models considered in this report are presented in Section 2.1. The mathematical form of the FEH model is discussed in Section 2.2.

\subsection{Mixture Models}

Many model forms have been proposed in the statistics literature for modeling mixture properties as functions of composition. Cornell ${ }^{2}$ discusses many of these mixture model forms, the most widely used being the first- and second-order canonical polynomial models of Scheffé. As used in this report for modeling PCT elemental releases, the Scheffe first-order mixture (FOM) model is

$$
\ln (N R)=\sum_{i=1}^{q} b_{i} x_{i}
$$

and the Scheffé second-order mixture (SOM) model is

$$
\ln (N R)=\sum_{i=1}^{q} b_{i} x_{i}+\sum_{i=1}^{q-1} \sum_{j \geq i}^{q} b_{i j} x_{i} x_{j}
$$

In (1) and (2), $\ln (\mathrm{NR})$ is the natural logarithm of a normalized elemental release from the PCT $\left(\mathrm{g} / \mathrm{m}^{2}\right), q$ is the number of mixture (glass) components, $x_{i}$ is the mole fraction of the $\mathrm{i}$-th glass component such that $x_{1}+x_{2}+\ldots+x_{q}=1, b_{i}$ is the coefficient of the first-order blending term involving $x_{i}$, and $b_{i j}$ is the coefficient of the second-order blending term involving $x_{i}$ and $x_{j}$. The coefficients $b_{i}$ for $a$ first-order model or $b_{i}$ and $b_{i j}$ for a second-order model are determined by fitting a model to an appropriate set of glass composition and PCT release data using least squares regression. Third- and higher-order Scheffé mixture models exist, as do other non-polynomial mixture models ${ }^{2}$, but such models are not considered in this report.

Either mass or mole fractions of glass components may be used in models (1) and (2); both were used by Hrma, Piepel, et al. ${ }^{3}$ In this report, mole fractions are used to allow a direct comparison of mixture models with free energy of hydration models (which use mole fractions). Also, note that the full SOM model (2) does not normally contain squared terms. That is, normally $j>i$ in the $x_{i} x_{j}$ terms of $(2)$, so that $x_{i}^{2}$ terms (when $i=j$ ) are excluded. The squared terms are excluded when all $x_{i} x_{j}(j>i)$ terms are present, because otherwise the model would be overparameterized and unique coefficient estimates could not be obtained. However, reduced forms of (2) containing selected squared $\left(\mathrm{x}_{\mathrm{i}}^{2}\right)$ and/or crossproduct $\left(\mathrm{x}_{\mathrm{i}} \mathrm{x}_{\mathrm{j}}\right)$ terms can be used ${ }^{3,4}$. 


\subsection{Free Energy of Hydration Model}

The free energy of hydration (FEH) model $^{5}$ applied in this report has the form .

$$
\therefore \quad \ln (N R)=a+b\left\{\sum_{i * \mathrm{SiO}_{2}}^{k}\left(\Delta G_{i}\right) x_{i}+\left(\Delta G_{\mathrm{SOO}_{2}}\right)\left[x_{\mathrm{SiO}_{2}}-\sum_{i \in S}^{k} x_{i}\right]\right\}
$$

where $\ln (\mathrm{NR})$ and $\mathrm{x}_{\mathrm{i}}$ are as defined for (1) and (2), $\Delta \mathrm{G}_{\mathrm{i}}$ is the $\mathrm{FEH}$ value for the $\mathrm{i}$-th glass component ( $\mathrm{kcal} / \mathrm{mol}), \mathrm{k}$ is the number of glass components for which $\Delta \mathrm{G}_{\mathrm{i}}$ values are available, and " $a$ " and " $b$ " are coefficients determined by fitting the model (3) to a set of glass composition and PCT release data using least squares regression. The last summation sign in (3) is over each glass component $\mathrm{i}$ assumed to be associated with $\mathrm{SiO}_{2}$ in glass dissolution reactions, which is denoted $i \in S$. Further information about the FEH model can be found in the THERMO report by Jantzen et al. ${ }^{5}$. The component $F E H$ values $\Delta \mathrm{G}_{\mathrm{i}}$ used in this work were current as of early 1995 , and are documented in Table 1.

\subsection{Numbers of Components and Logarithm of Durability}

Note that the number of glass components included in a FEH model is denoted by $\mathrm{k}$ in (3), whereas the number of glass components in FOM and SOM models is denoted by $q$ in (1) and (2). The FEH approach accounts for the effects of many glass components, as indicated in Table I. In practice, however, many minor components have little or no practical effect on PCT normalized releases. The mixture models (1) and (2) can accommodate this by combining minor components in a catch-all component Others. The number of terms in mixture models can also be reduced by combining components with nearly identical effects, or by removing components with negligible effects (and renormalizing the composition to a total mole fraction of unity). Hence, while it is possible (assuming an adequate data set) to fit a mixture model using all k components used by the FEH approach, this is usually not done. In practice, very good mixture models can be obtained with $\mathrm{q}<\mathrm{k}$ (sometimes $\mathrm{q}<<\mathrm{k}$ ).

The natural logarithm of normalized elemental release is used in Eqs. (1), (2), and (3) for two reasons. First, a logarithmic transformation of the dependent variable in a regression model (normalized PCT release in this report) stabilizes the variances of measured values when the variances increase with the magnitudes of measured values. Stabilized variances are a requirement for unweighted least squares regression. Second, a natural logarithmic transformation (log base e) is preferred over a common logarithmic transformation (log base 10) because of the relationship

$$
\mathrm{SD}[\ln (\mathrm{NR})] \approx \mathrm{RSD}[\mathrm{NR}]
$$

where SD denotes "standard deviation" and RSD denotes "relative standard deviation". Thus, a standard deviation in $\ln (\mathrm{NR})$ units can be interpreted as a relative standard deviation in NR units. This fact will be used in Sections 5 and 7. 
Table 1. Component Free Energy of Hydration Values $\left(\Delta G_{i}\right)$ Used In This Work

\begin{tabular}{|c|c|c|c|c|c|}
\hline Oxide (i) & $\begin{array}{c}\Delta \mathrm{G}_{\mathrm{i}} \\
(\mathrm{kcal} / \mathrm{mol})\end{array}$ & Oxide (i) & $\begin{array}{c}\Delta \mathrm{G}_{\mathrm{i}} \\
(\mathrm{kcal} / \mathrm{mol})\end{array}$ & Oxide (i) & $\begin{array}{c}\Delta \mathrm{G}_{\mathrm{i}} \\
(\mathrm{kcal} / \mathrm{mol})\end{array}$ \\
\hline $\mathrm{Ag}_{2} \mathrm{O}$ & 0.0 & $\mathrm{~K}_{2} \mathrm{O}$ & $-\mathrm{xx} . \mathrm{xx}$ & $\mathrm{Rh}_{2} \mathrm{O}_{3}$ & 0.0 \\
\hline $\mathrm{Al}_{2} \mathrm{O}_{3}$ & $\mathbf{x x} . \mathbf{x x}$ & $\mathrm{La}_{2} \mathrm{O}_{3}$ & $-\mathrm{xx} \cdot \mathrm{xx}$ & $\mathrm{RuO}_{2}$ & $\mathrm{xx} . \mathrm{x}$ \\
\hline $\mathrm{As}_{2} \mathrm{O}_{3}$ & $-x \dot{x} \cdot x x$ & $\mathrm{Li}_{2} \mathrm{O}$ & $-\mathrm{xx} . \mathrm{xx}$ & $\mathrm{Sb}_{2} \mathrm{O}_{3}$ & $-\mathrm{xx} . \mathrm{xx}$ \\
\hline $\mathrm{B}_{2} \mathrm{O}_{3}$ & $-x x . x x$ & $\mathrm{MgO}$ & $-\mathrm{x} . \mathrm{Xx}$ & $\mathrm{SeO}_{2}$ & $-\mathrm{x} . \mathrm{x}$ \\
\hline $\mathrm{BaO}$ & $-x x \cdot x x$ & $\mathrm{MnO}$ & $-x x . x x$ & $\mathrm{SiO}_{2}$ & $\mathbf{X} . \mathrm{XX}$ \\
\hline $\mathrm{Bi}_{2} \mathrm{O}_{3}$ & 0.0 & $\mathrm{MoO}_{3}$ & $\mathrm{xx} . \mathrm{xx}$ & $\mathrm{Sm}_{2} \mathrm{O}_{3}$ & 0.0 \\
\hline $\mathrm{CaO}$ & $-x . x x$ & $\mathrm{Na}_{2} \mathrm{O}$ & $-x x . x x$ & $\mathrm{SO}_{3}$ & 0.0 \\
\hline $\mathrm{CdO}$ & $\mathbf{x} . \mathbf{x x}$ & $\mathrm{Nd}_{2} \mathrm{O}_{3}$ & $-x x . x x$ & $\mathrm{SrO}$ & $-\mathrm{xx} . \mathrm{xx}$ \\
\hline $\mathrm{Ce}_{2} \mathrm{O}_{3}$ & $-\mathrm{xx} . \mathrm{xx}$ & $\mathrm{NiO}$ & $\mathrm{X} . \mathrm{xx}$ & $\mathrm{TeO}_{2}$ & $\mathrm{Xx} . \mathrm{Xx}$ \\
\hline $\mathrm{CoO}$ & $-\mathrm{x} . \mathrm{xx}$ & $\mathrm{P}_{2} \mathrm{O}_{5}$ & $-x x . x x$ & $\mathrm{ThO}_{2}$ & $\mathbf{x x} . \mathbf{x x}$ \\
\hline $\mathrm{Cr}_{2} \mathrm{O}_{3}$ & $\mathbf{x x} . \mathrm{xx}$ & $\mathrm{PbO}$ & $x x \cdot x x$ & $\mathrm{TiO}_{2}$ & $\mathbf{x x} . \mathbf{x x}$ \\
\hline $\mathrm{Cs}_{2} \mathrm{O}$ & $-\mathrm{xx} . \mathrm{xx}$ & PdO & 0.0 & $\mathrm{U}_{3} \mathrm{O}_{8}$ & $-\mathrm{xx} . \mathrm{xx}$ \\
\hline $\mathrm{CuO}$ & $-\mathbf{x} . \mathbf{x x}$ & $\mathrm{PdO}_{2}$ & 0.0 & $\mathrm{WO}_{3}$ & 0.0 \\
\hline F & 0.0 & $\mathrm{Pr}_{6} \mathrm{O}_{11}$ & 0.0 & $\mathrm{Y}_{2} \mathrm{O}_{3}$ & $-\mathrm{xx} \cdot \mathrm{xx}$ \\
\hline $\mathrm{FeO}$ & $-\mathrm{xx} \cdot \mathrm{xx}$ & $\mathrm{Rb}_{2} \mathrm{O}$ & $-x x . x x$ & $\mathrm{ZnO}$ & $x . x x$ \\
\hline $\mathrm{Fe}_{2} \mathrm{O}_{3}$ & $x x \cdot x x$ & $\mathrm{RhO}_{2}$ & 0.0 & $\mathrm{ZrO}_{2}$ & $x x \cdot x x$ \\
\hline
\end{tabular}

Note 1: Jantzen et al. ${ }^{5}$ and various information therein has not yet been cleared for public release or reference. Hence, the component FEH values and designators of which components occur in silicate species could not be included in this report. If and when release of this information is authorized, a completed version of this table will be made available to those requesting it from the first author.

Note 2: $\Delta \mathrm{G}_{\mathrm{i}}=0.0$ where values were not given or were given as zero by Jantzen et al. ${ }^{5}$. 



\section{Comparing Mixture and Free Energy of Hydration Model Forms}

Section 3.1 shows how a FEH model can be rewritten in the form of a FOM model so that their coefficients can be compared. Section 3.2 discusses two approaches for deriving quantities from FOM models that can be compared to the component free energies of hydration used in FEH models. Section 3.3 discusses a hybrid FEH/FOM modeling approach. Section 3.4 discusses the results in the other subsections.

\subsection{Writing the FEH Model in the Form of a FOM Model}

The FOM model in Eq. (1) and the FEH model in Eq. (3) are both seen to be first-order functions of composition. To facilitate comparing FEH and FOM models and their coefficients later in the report, this section shows how the FEH model may be rewritten in the form of the FOM model.

By moving the constant $b$ inside the summation, the FEH model (3) may be written as

$$
\ln (N R)=a+\sum_{i * S O_{2}}^{k} b\left(\Delta G_{i}\right) x_{i}+b\left(\Delta G_{\mathrm{SiO}_{2}}\right) \cdot\left[x_{\mathrm{SiO}_{2}}-\sum_{i \in S}^{k} x_{i}\right] .
$$

Collecting the $\Delta \mathrm{G}_{i} \mathrm{x}_{\mathrm{i}}$ terms yields

$$
\ln (N R)=a+\sum_{i=1}^{k} b\left(\Delta G_{i}\right) x_{i}-b\left(\Delta G_{S O_{2}}\right) \sum_{i \in S}^{k} x_{i} .
$$

Assuming the complete composition of each glass is known such that $\Sigma x_{i}=1$, and multiplying the constant $a$ by $1=\Sigma \mathrm{x}_{i}$, yields

$$
\begin{aligned}
\ln (N R) & =\sum_{i=1}^{k} a x_{i}+\sum_{i=1}^{k} b\left(\Delta G_{i}\right) x_{i}-b\left(\Delta G_{S O_{2}}\right) \sum_{i \in S}^{k} x_{i} \\
& =\sum_{i=1}^{k}\left[a+b\left(\Delta G_{i}\right)\right] x_{i}-b\left(\Delta G_{S O_{2}}\right) \sum_{i \in S}^{k} x_{i} .
\end{aligned}
$$

Finally, collecting terms according to whether each component i falls in the silica association group $(\mathrm{i} \in S$ ) or in the non-silica association group ( $\mathrm{i} \notin S$ ) yields

$$
\begin{aligned}
\ln (N R) & =\sum_{i \in S}^{k}\left[a+b\left(\Delta G_{i}\right)\right] x_{i}+\sum_{i \in S}^{k}\left[a+b\left(\Delta G_{i}-\Delta G_{S i O_{2}}\right)\right] x_{i} \\
& =\sum_{i \in S}^{k} c_{i} x_{i}+\sum_{i \in S}^{k} d_{i} x_{i}
\end{aligned}
$$

where $c_{i}=a+b\left(\Delta G_{i}\right)$ and $d_{i}=a+b\left(\Delta G_{i}-\Delta G_{\mathrm{SO}_{2}}\right)$. Thus, the rewritten FEH model in 
(5d) is seen to be of the same general form as the FOM model in (1), except that the terms fall into two groups (depending on whether or not each component is assumed to be associated with $\mathrm{SiO}_{2}$ in glass dissolution reactions).

For the FOM model (1) and the FEH model (3) fitted to the same data set via least squares regression, the $b_{i}$ coefficient for each component from (1) can be compared with the corresponding coefficient $c_{i}$ or $d_{i}$ from the rewritten FEH model in (5d).

\subsection{Deriving Coefficients from a FOM Model That Can Be Compared to Component Free Energy of Hydration Values}

FEH and FOM models can also be compared by deriving coefficients $\Delta \mathrm{G}_{\mathrm{i}}^{\prime}$ from a FOM model and comparing them to the $\Delta \mathrm{G}_{i}$ component FEH values in Table 1 . The $\Delta \mathrm{G}_{i}^{\prime}$ coefficients may be thought of as "data-determined" component FEH values. Two ways to derive such $\Delta \mathrm{G}_{i}^{\prime}$ coefficients are presented in this section.

One way to obtain $\Delta G_{i}^{\prime}$ coefficients from a FOM model (1) fitted to a data set requires that the FEH model (3) also be fitted to the data set. Then, the $b_{i}$ values from (1) and the $a$ and $b$ values from (3) are substituted into the coefficient relationships in (5d), which are then solved for $\Delta \mathrm{G}_{\mathrm{i}}^{\prime}$. This yields

$$
\begin{gathered}
\Delta G_{i}^{\prime}=\frac{b_{i}-a}{b} . \text { for } i \notin S \\
\Delta G_{i}^{\prime}=\Delta G_{S_{S O_{2}}^{\prime}+\frac{b_{i}-a}{b} \text { for } i \in S} \text { foS }
\end{gathered}
$$

Because $\mathrm{SiO}_{2}$ is not contained in the set $S$, Eq. (6a) is used to obtain $\Delta \mathrm{G}_{\mathrm{SiO}}^{\prime}$, which can then be used in determining the quantities in Eq. (6b). Note that in the case where a FOM model uses $q<k$ components, where $x_{q}=x_{q}+x_{q+1}+\ldots+x_{k}$, the $\Delta G_{i}^{\prime}$ values for $i=q, q+1, \ldots, k$ are the same. The approach in (6a) and (6b) for deriving $\Delta \mathrm{G}_{\mathrm{i}}^{\mathrm{i}}$ values from the fitted FOM model (1) coefficients relies on the assumption that the $a$ and $b$ coefficients from the fitted FEH model (3) are appropriate.

Another way to obtain $\Delta \mathrm{G}_{\mathrm{i}}^{\prime}$ coefficients from a FOM model (1) fitted to a data set is to assume that two or three of the $\Delta G_{i}$ values in Table 1 are applicable (or correct) for the data set (i.e., $\Delta \mathrm{G}_{\mathrm{i}}^{\prime}=\Delta \mathrm{G}_{\mathrm{i}}$ for component i). Substituting the two or three $\Delta \mathrm{G}_{\mathrm{i}}$ values assumed to be correct into Eqs. (6a) and (6b) allows solving for the values of $a$ and $b$. Then, the remaining $\Delta \mathrm{G}_{\mathrm{i}}^{\prime}$ coefficients can be derived from the FOM model coefficients using Eqs. (6a) and (6b). Whether two or three $\Delta G_{i}$ values must be assumed applicable (or correct) to uniquely solve for each $\Delta G_{i}^{\prime}$ depends on whether the corresponding glass components are in the group $S$ or not. Several cases are considered below. 
Case 1: Two components $\mathrm{j}$ and $\mathrm{k}$ are sufficient if $\mathrm{j} \notin S$ and $\mathrm{k} \notin S$. This includes the case where neither of $\mathrm{j}$ or $\mathrm{k}$ is $\mathrm{SiO}_{2}$, as well as the cases where one of $\mathrm{j}$ or $\mathrm{k}$ is $\mathrm{SiO}_{2}$. Simultaneously solving the corresponding Eqs. (6a) for $j$ and $k$ yields

$$
\begin{aligned}
& b=\frac{b_{j}-b_{k}}{\Delta G_{j}-\Delta G_{k}} \\
& a=b_{k}-b \Delta G_{k}
\end{aligned}
$$

Then, $\Delta \mathrm{G}_{\mathrm{j}}^{\prime}=\Delta \mathrm{G}_{\mathrm{j}}, \Delta \mathrm{G}_{\mathrm{k}}^{\prime}=\Delta \mathrm{G}_{\mathrm{k}}$, and the remaining $\Delta \mathrm{G}_{\mathrm{i}}^{\prime}$ for all $\mathrm{i} \neq \mathrm{j}, \mathrm{k}$ are obtained by substituting the above values of $a$ and $b$ into Eqs. (6a) and (6b).

Case 2: Two components are also sufficient if $\mathrm{j} \in \mathrm{S}$ and $\mathrm{k}=\mathrm{SiO}_{2}$ (without loss of generality, since the names of $j$ and $k$ could be switched). Simultaneously solving the corresponding Eq. (6b) for $j$ and Eq. (6a) for $k$ yields

$$
\begin{aligned}
& b=\frac{b_{j}-b_{\mathrm{SiO}_{2}}}{\Delta G_{j}-2 \Delta G_{\mathrm{SiO}_{2}}} \\
& a=b_{\mathrm{SiO}_{2}}-b \Delta G_{\mathrm{SiO}_{2}}
\end{aligned}
$$

Then, $\Delta \mathrm{G}_{\mathrm{j}}^{\prime}=\Delta \mathrm{G}_{\mathrm{j}}, \Delta \mathrm{G}_{\mathrm{SiO2}}^{\prime}=\Delta \mathrm{G}_{\mathrm{SiO2}}$, and the remaining $\Delta \mathrm{G}_{\mathrm{i}}^{\prime}$ for all $\mathrm{i} \neq \mathrm{j}$ or $\mathrm{SiO}_{2}$, are obtained by substituting the above values of $a$ and $b$ into Eqs. (6a) and (6b).

Case 3: Three components $\mathrm{j}, \mathrm{k}$, and $\mathrm{h}$ are sufficient if $\mathrm{j} \notin S$ (where $\mathrm{j} \neq \mathrm{SiO}_{2}$ ), $\mathrm{k} \in S$, and $\mathrm{h} \in S$ (without loss of generality, since the names of $\mathrm{j}, \mathrm{k}$, and $\mathrm{h}$ could be switched). Simultaneously solving the corresponding Eq. (6a) for $j$ and Eqs. (6b) for $k$ and $h$ yields

$$
\begin{gathered}
b=\frac{b_{k}-b_{h}}{\Delta G_{k}-\Delta G_{h}} \\
a=b_{j}-b \Delta G_{j} \\
\Delta G_{S i O_{2}}^{\prime}=\Delta G_{h}-\frac{b_{h}-a}{b}
\end{gathered}
$$

Then, $\Delta \mathrm{G}_{\mathrm{j}}^{\prime}=\Delta \mathrm{G}_{\mathrm{j},}, \Delta \mathrm{G}_{\mathrm{k}}^{\prime}=\Delta \mathrm{G}_{\mathrm{k},}, \Delta \mathrm{G}_{\mathrm{h}}^{\prime}=\Delta \mathrm{G}_{\mathrm{h}}$ and the remaining $\Delta \mathrm{G}_{\mathrm{i}}^{\prime}$ for all $\mathrm{i} \neq \mathrm{j}, \mathrm{k}$, h are obtained by substituting the above values of $a, b$, and $\Delta \mathrm{G}_{\mathrm{SiO} 2}^{\prime}$ into Eqs. (6a) and (6b). 
Any other combinations of two or three components either revert to one of the three cases above, or do not lead to a solution.

\subsection{A Hybrid FEH/FOM Modeling Approach}

A hybrid FEH/FOM modeling approach could be used. This would involve fitting (to a given data set of glass composition and PCT release data) a model of the form

$$
\ln (N R)=\sum_{i \in S}^{k} g_{i}\left(\Delta G_{i}\right) x_{i}+\sum_{i \in S}^{k} g_{i}\left(\Delta G_{i}-\Delta G_{S O_{2}}\right) x_{i}
$$

where $\ln (N R), x_{i}, \Delta G_{i}$, and $k$ are as defined in Section 2 , and $g_{i}$ is the coefficient for the $i$-th glass component obtained as part of the least squares fit. Eq. (10) is a modification of Eq. (5d), where $c_{i}=g_{i}\left(\Delta G_{i}\right)$ and $d_{i}=g_{i}\left(\Delta G_{i}-\Delta G_{\text {SiO2 }}\right)$ would be the coefficients of the FOM model. In this model, a $\mathrm{g}_{\mathrm{i}}$ coefficient may be thought of as a multiplicative factor that "adjusts" the assumed FEH value $\left[\left(\Delta \mathrm{G}_{\mathrm{i}}\right)\right.$ or $\left.\left(\Delta \mathrm{G}_{\mathrm{i}}-\Delta \mathrm{G}_{\mathrm{SiO} 2}\right)\right]$ for component $\mathrm{i}$ for a particular data set (and the propertycomposition space it represents). $A g_{i}$ value that is (is not) statistically different from 1.0 would indicate that the corresponding FEH value is not (is) reasonable for the particular data set.

\subsection{Discussion}

Only the results from Section 3.1 are used in the remainder of this report. It was beyond the scope of this work to apply the results presented in Sections 3.2 and 3.3 to the data sets discussed in Section 4. However, it was decided to include Sections 3.2 and 3.3 in this report so that the results would be documented for possible future use.

The main result from Section 3.1 used in the remainder of this report is that the FEH model [(3) rewritten as (5d)] has the same form as the FOM model (1). The main difference between the two models is that the FEH model assumes that $\mathrm{FEH}$ values for each component are applicable component coefficients, whereas the FOM model estimates component coefficients from experimental data. Differences between assumed and estimated component coefficients for a given data set may indicate that the assumed reactions leading to the particular FEH coefficient values are not appropriate for the glass composition space represented by the data set. Such indications may be useful in improving the theory or assumptions underlying the FEH model.

An additional advantage of the mixture approach is that it can account for second-order (or higher-order) effects (both curvature and interactions) of glass components by including squared terms and crossproduct terms in the model [as in (2)]. The FEH modeling approach presented in Section 2.2 (referred to as "preliminary" FEH by Jantzen et al. ${ }^{5}$ ) cannot account for second-order effects of components in this fashion. Jantzen et al. ${ }^{5}$ discuss a modification of the "preliminary" FEH approach (referred to as "final" $F E H$ ) that essentially allows for adjusting the $\Delta G_{i}$ values in Table 1 based on pH effects on PCT releases. However, the work summarized in this report was well under way prior to the completion by Jantzen et al. of the "final" FEH approach. Hence, only the "preliminary" and not the "final" FEH modeling approach is compared to the mixture modeling approach in this report. 


\section{Data Sets}

Several data sets (glass compositions in terms of mole fractions, and PCT normalized B releases) from the waste glass literature are used to compare the fitting and predictive abilities of mixture and FEH models. The data sets are briefly described in the following subsections. Listings of the mole fraction compositions and PCT normalized B releases for each data set are given in Appendix A.

The glass mole fraction compositions given in Appendix A for the various data sets required (in some cases) adjustments from the way they were reported in the documents from which they were taken. For most data sets, the compositions were reported in terms of mass fractions or mass percents, and had to be converted to mole fractions. In some data sets, the oxide forms of a small number of components were different than those assumed by the $\mathrm{FEH}$ approach (e.g., $\mathrm{MnO}_{2}$ instead of $\mathrm{MnO}$ ), and had to be converted to the FEH-assumed oxide forms.

Normalized B releases from the 7-day Product Consistency Test (PCT) are used in this report as indicators of glass durability. The PCT exposes crushed glass to deionized water at $90^{\circ} \mathrm{C}$ for seven days, and is responsive to both glass homogeneity and composition ${ }^{1}$ :

Normalized B releases in this report were obtained via the formula

$$
N R=\frac{C}{f \cdot \frac{A}{V}}
$$

where NR is the normalized B release $\left(\mathrm{g} / \mathrm{m}^{2}\right), C$ is the concentration of $B$ in the PCT solution $(\mathrm{mg} / \mathrm{L}), \mathrm{f}$ is the mass fraction of $B$ in the glass (unitless), and $A / V$ is the surface area to volume ratio for the PCT (assumed to be $2000 \mathrm{~m}^{-1}$ for this work). Boron release is commonly selected as a good indicator of PCT durability of borosilicate waste glasses for the following reasons: 1) all B dissolved from glass enters into solution and does not precipitate in the gel layer, 2) B is released only by dissolution of the glass network, not by a diffusion process, and 3) B concentration in solution is determined with very good analytical precision.

Appendix B contains scatterplot matrices of the glass mole fraction compositions for each data set. A scatterplot matrix is simply a matrix of scatterplots of all possible pairs of components. Because individual plots in a scatterplot matrix get quite small for large numbers of components, attention was limited to only those components used in the FOM model for each data set. Observations regarding scatterplot matrices for the various data sets are made in the following subsections.

Table 2 lists the ranges of the component mole fraction values and the range of normalized $B$ release values for each of the data sets discussed below. These ranges provide a rough indication of the composition region and the range of normalized $B$ releases covered by each data set. 
Table 2. Ranges of Glass Components and PCT Normalized B Release

\begin{tabular}{|c|c|c|c|c|c|c|c|}
\hline \multicolumn{2}{|c|}{ Component } & \multirow{2}{*}{$\begin{array}{l}\text { CVS-I } \\
.0000 \\
.0930 \\
\end{array}$} & \multirow{2}{*}{$\begin{array}{c}\text { CVS-II.1 } \\
.0062 \\
.0690 \\
\end{array}$} & \multirow{2}{*}{$\begin{array}{c}\text { CVS-II.2 } \\
.0000 \\
.0885\end{array}$} & \multirow{2}{*}{$\begin{array}{c}\text { CVS-II.3 } \\
.0112 \\
.1269\end{array}$} & \multirow{2}{*}{$\begin{array}{c}\text { CVS-II.4 } \\
.0000 \\
.1004\end{array}$} & \multirow{2}{*}{$\begin{array}{c}\text { CVS (all) } \\
.0000 \\
.1269 \\
\end{array}$} \\
\hline $\mathrm{Al}_{2} \mathrm{O}_{3}$ & $\begin{array}{l}\text { Low } \\
\text { High }\end{array}$ & & & & & & \\
\hline $\mathrm{B}_{2} \mathrm{O}_{3}$ & $\begin{array}{l}\text { Low } \\
\text { High }\end{array}$ & $\begin{array}{l}.0414 \\
.1953 \\
\end{array}$ & $\begin{array}{r}.0629 \\
.1648 \\
\end{array}$ & $\begin{array}{r}.0420 \\
.1974 \\
\end{array}$ & $\begin{array}{r}.0467 \\
.1661 \\
\end{array}$ & $\begin{array}{l}.0465 \\
.1882 \\
\end{array}$ & $\begin{array}{l}.0414 \\
.1974 \\
\end{array}$ \\
\hline $\mathrm{BaO}$ & $\begin{array}{l}\text { Low } \\
\text { High }\end{array}$ & (b) & (b) & (b) & (b) & (b) & (b) \\
\hline $\mathrm{CaO}$ & $\begin{array}{l}\text { Low } \\
\text { High }\end{array}$ & $\begin{array}{l}.0000 \\
.1227 \\
\end{array}$ & $\begin{array}{l}.0000 \\
.0817 \\
\end{array}$ & $\begin{array}{l}.0000 \\
.0968 \\
\end{array}$ & $\begin{array}{l}.0000 \\
.1201 \\
\end{array}$ & $\begin{array}{l}.0086 \\
.0232 \\
\end{array}$ & $\begin{array}{r}.0000 \\
.1227 \\
\end{array}$ \\
\hline $\mathrm{Cr}_{2} \mathrm{O}_{3}$ & $\begin{array}{l}\text { Low } \\
\text { High }\end{array}$ & (b) & (b) & (b) & (b) & (b) & (b) \\
\hline $\mathrm{CuO}$ & $\begin{array}{l}\text { Low } \\
\text { High }\end{array}$ & (b) & (b) & (b) & (b) & (b) & (b) \\
\hline $\mathrm{Fe}_{2} \mathrm{O}_{3}$ & $\begin{array}{l}\text { Low } \\
\text { High }\end{array}$ & $\begin{array}{l}.0072 \\
.0626 \\
\end{array}$ & $\begin{array}{l}.0154 \\
.0492 \\
\end{array}$ & $\begin{array}{l}.0072 \\
.0596 \\
\end{array}$ & $\begin{array}{l}.0002 \\
.0513 \\
\end{array}$ & & $\begin{array}{l}.0002 \\
.0626 \\
\end{array}$ \\
\hline $\mathrm{FeO}$ & $\begin{array}{l}\text { Low } \\
\text { High }\end{array}$ & (a) & (a) & (a) & (a) & (a) & (a) \\
\hline$\overline{\mathrm{K}_{2} \mathrm{O}}$ & $\begin{array}{l}\text { Low } \\
\text { High }\end{array}$ & (a) & (a) & (a) & (a) & (a) & (a) \\
\hline $\mathrm{Li}_{2} \mathrm{O}$ & $\begin{array}{l}\text { Low } \\
\text { High }\end{array}$ & $\begin{array}{l}.0200 \\
.1526 \\
\end{array}$ & $\begin{array}{l}.0448 \\
.1294 \\
\end{array}$ & $\begin{array}{l}.0209 \\
.1639 \\
\end{array}$ & $\begin{array}{l}.0115 \\
.1518 \\
\end{array}$ & $\begin{array}{l}.0225 \\
.1466 \\
\end{array}$ & $\begin{array}{l}.0115 \\
.1639 \\
\end{array}$ \\
\hline $\mathrm{MgO}$ & $\begin{array}{l}\text { Low } \\
\text { High }\end{array}$ & $\begin{array}{l}.0000 \\
.1343 \\
\end{array}$ & $\begin{array}{l}.0000 \\
.0779 \\
\end{array}$ & $\begin{array}{l}.0000 \\
.1288 \\
\end{array}$ & $\begin{array}{l}.0000 \\
.0668 \\
\end{array}$ & $\begin{array}{l}.0000 \\
.0320 \\
\end{array}$ & $\begin{array}{l}.0000 \\
.1343 \\
\end{array}$ \\
\hline $\mathrm{MnO}$ & $\begin{array}{l}\text { Low } \\
\text { High }\end{array}$ & (b) & (b) & (b) & (b) & (b) & (b) \\
\hline $\mathrm{Na}_{2} \mathrm{O}$ & $\begin{array}{l}\text { Low } \\
\text { High }\end{array}$ & $\begin{array}{l}.0484 \\
.2043 \\
\end{array}$ & $\begin{array}{l}.0696 \\
.1804 \\
\end{array}$ & $\begin{array}{l}.0451 \\
.2110 \\
\end{array}$ & $\begin{array}{l}.0518 \\
.2077 \\
\end{array}$ & $\begin{array}{l}.0527 \\
.2089 \\
\end{array}$ & $\begin{array}{l}.0451 \\
.2110 \\
\end{array}$ \\
\hline $\mathrm{NiO}$ & $\begin{array}{l}\text { Low } \\
\text { High }\end{array}$ & (b) & (b) & (b) & (b) & (b) & (b) \\
\hline $\mathrm{P}_{2} \mathrm{O}_{5}$ & $\begin{array}{l}\text { Low } \\
\text { High }\end{array}$ & (b) & (b) & (b) & (b) & (b) & (b) \\
\hline $\mathrm{SiO}_{2}$ & $\begin{array}{l}\text { Low } \\
\text { High }\end{array}$ & $\begin{array}{l}.4194 \\
.6417 \\
\end{array}$ & $\begin{array}{l}.4913 \\
.5849 \\
\end{array}$ & $\begin{array}{l}.3905 \\
.6208 \\
\end{array}$ & $\begin{array}{l}.3623 \\
.6266 \\
\end{array}$ & $\begin{array}{l}.4127 \\
.6225 \\
\end{array}$ & $\begin{array}{l}.3623 \\
.6417 \\
\end{array}$ \\
\hline $\mathrm{ThO}_{2}$ & $\begin{array}{l}\text { Low } \\
\text { High }\end{array}$ & (a) & (a) & (a) & (a) & (a) & (a) \\
\hline $\mathrm{TiO}_{2}$ & $\begin{array}{l}\text { Low } \\
\text { High }\end{array}$ & (a) & (a) & (a) & (a) & (a) & (a) \\
\hline $\mathrm{U}_{3} \mathrm{O}_{8}$ & $\begin{array}{l}\text { Low } \\
\text { High }\end{array}$ & (a) & (a) & (b) & (a) & (a) & (b) \\
\hline $\mathrm{ZrO}_{2}$ & $\begin{array}{l}\text { Low } \\
\text { High }\end{array}$ & $\begin{array}{l}.0000 \\
.0717 \\
\end{array}$ & $\begin{array}{l}.0050 \\
.0523 \\
\end{array}$ & $\begin{array}{l}.0000 \\
.0671 \\
\end{array}$ & $\begin{array}{l}.0000 \\
.0792 \\
\end{array}$ & & $\begin{array}{l}.0000 \\
.0792 \\
\end{array}$ \\
\hline Others & $\begin{array}{l}\text { Low } \\
\text { High }\end{array}$ & $\begin{array}{l}.0054 \\
.0624 \\
\end{array}$ & $\begin{array}{l}.0140 \\
.0485 \\
\end{array}$ & $\begin{array}{l}.0052 \\
.0615 \\
\end{array}$ & $\begin{array}{l}.0054 \\
.0561 \\
\end{array}$ & $\begin{array}{l}.0758 \\
.1181 \\
\end{array}$ & $\begin{array}{r}.0052 \\
.0624 \\
\end{array}$ \\
\hline $\begin{array}{l}\text { PCT B } \\
\left(\mathrm{g} / \mathrm{m}^{2}\right)\end{array}$ & $\begin{array}{l}\text { Low } \\
\text { High }\end{array}$ & $\begin{array}{r}0.066 \\
20.639 \\
\end{array}$ & $\begin{array}{l}0.128 \\
2.937 \\
\end{array}$ & $\begin{array}{r}0.173 \\
12.701 \\
\end{array}$ & $\begin{array}{r}0.115 \\
44.000 \\
\end{array}$ & $\begin{array}{r}0.193 \\
14.400 \\
\end{array}$ & $\begin{array}{r}0.066 \\
44.000 \\
\end{array}$ \\
\hline
\end{tabular}

(a) Component not included or determined for glasses in this data set.

(b) Component included in Others for this data set. 
Table 2. Ranges of Glass Components and PCT Normalized B Release (cont.)

\begin{tabular}{|c|c|c|c|c|c|c|c|c|}
\hline \multicolumn{2}{|c|}{ Component } & \multirow{2}{*}{$\begin{array}{l}\text { Ramsey } \\
.0012 \\
.1568 \\
\end{array}$} & \multirow{2}{*}{$\begin{array}{l}\text { WV87 } \\
.0630 \\
.0924\end{array}$} & \multirow{2}{*}{$\begin{array}{l}\text { WV88 } \\
.0370 \\
.0737 \\
\end{array}$} & \multirow{2}{*}{$\begin{array}{r}\text { WVDP } \\
.0294 \\
.0612 \\
\end{array}$} & \multirow{2}{*}{$\begin{array}{c}\text { SRTC } 137 \\
.0012 \\
.1016 \\
\end{array}$} & \multirow{2}{*}{$\begin{array}{c}\text { SRTC } 118 \\
.0086 \\
.0885 \\
\end{array}$} & \multirow{2}{*}{$\begin{array}{c}\text { SRTC 111 } \\
.0196 \\
.0885 \\
\end{array}$} \\
\hline $\mathrm{Al}_{2} \mathrm{O}_{3}$ & $\begin{array}{l}\text { Low } \\
\text { High }\end{array}$ & & & & & & & \\
\hline $\mathrm{B}_{2} \mathrm{O}_{3}$ & $\begin{array}{l}\text { Low } \\
\text { High }\end{array}$ & $\begin{array}{l}.0409 \\
.1543 \\
\end{array}$ & $\begin{array}{l}.1017 \\
.1375 \\
\end{array}$ & $\begin{array}{l}.0707 \\
.1313 \\
\end{array}$ & $\begin{array}{l}.0723 \\
.2000 \\
\end{array}$ & $\begin{array}{l}.0491 \\
.1428 \\
\end{array}$ & $\begin{array}{l}.0553 \\
.1281 \\
\end{array}$ & $\begin{array}{l}.0553 \\
.1281 \\
\end{array}$ \\
\hline $\mathrm{BaO}$ & $\begin{array}{l}\text { Low } \\
\text { High }\end{array}$ & (a) & $\begin{array}{l}.0152^{\left({ }^{(c)}\right.} \\
.0446^{(\mathrm{c})} \\
\end{array}$ & $\begin{array}{l}.0082^{(c)} \\
.0418^{(c)} \\
\end{array}$ & (b) & (b) & (b) & (b) \\
\hline $\mathrm{CaO}$ & $\begin{array}{l}\text { Low } \\
\text { High }\end{array}$ & $\begin{array}{l}.0000 \\
.1040 \\
\end{array}$ & $\begin{array}{l}.0152^{(\mathrm{c})} \\
.0446^{(\mathrm{c})} \\
\end{array}$ & $\begin{array}{l}.0082^{(c)} \\
.0418^{\left(\frac{)}{2}\right.}\end{array}$ & (b) & $\begin{array}{l}.0000 \\
.1027 \\
\end{array}$ & $\begin{array}{l}.0044 \\
.0180 \\
\end{array}$ & $\begin{array}{l}.0044 \\
.0180 \\
\end{array}$ \\
\hline $\mathrm{Cr}_{2} \mathrm{O}_{3}$ & $\begin{array}{l}\text { Low } \\
\text { High }\end{array}$ & (a) & (b) & (b) & (b) & $\begin{array}{l}.0000 \\
.0028 \\
\end{array}$ & $\begin{array}{l}.0000 \\
.0028 \\
\end{array}$ & $\begin{array}{l}.0000 \\
.0028 \\
\end{array}$ \\
\hline $\mathrm{CuO}$ & $\begin{array}{l}\text { Low } \\
\text { High }\end{array}$ & (a) & (b) & (b) & (b) & $\begin{array}{l}.0000 \\
.0054 \\
\end{array}$ & $\begin{array}{l}.0000 \\
.0054 \\
\end{array}$ & $\begin{array}{l}.0000 \\
.0037 \\
\end{array}$ \\
\hline $\mathrm{Fe}_{2} \mathrm{O}_{3}$ & $\begin{array}{l}\text { Low } \\
\text { High }\end{array}$ & $\begin{array}{l}.0000 \\
.0975 \\
\end{array}$ & $\begin{array}{l}.0379 \\
.0685 \\
\end{array}$ & $\begin{array}{l}.0419 \\
.0738 \\
\end{array}$ & $\begin{array}{l}.0294 \\
.0857 \\
\end{array}$ & $\begin{array}{l}.0000 \\
.0600 \\
\end{array}$ & $\begin{array}{l}.0175 \\
.0600 \\
\end{array}$ & $\begin{array}{l}.0175 \\
.0600 \\
\end{array}$ \\
\hline $\mathrm{FeO}$ & $\begin{array}{l}\text { Low } \\
\text { High }\end{array}$ & $\begin{array}{l}.0000 \\
.0340\end{array}$ & (a) & (a) & (a) & $\begin{array}{l}.0000 \\
.0789 \\
\end{array}$ & $\begin{array}{l}.0000 \\
.0790 \\
\end{array}$ & $\begin{array}{l}.0000 \\
.0127 \\
\end{array}$ \\
\hline $\mathrm{K}_{2} \mathrm{O}$ & $\begin{array}{l}\text { Low } \\
\text { High }\end{array}$ & (a) & $\begin{array}{l}.1584^{(0)} \\
.2007^{(0)}\end{array}$ & $\begin{array}{l}.1697^{(0)} \\
.2493^{(0)}\end{array}$ & $\begin{array}{l}.0232 \\
.0591\end{array}$ & $\begin{array}{l}.0000 \\
.0395 \\
\end{array}$ & $\begin{array}{l}.0000 \\
.0395 \\
\end{array}$ & $\begin{array}{l}.0000 \\
.0270 \\
\end{array}$ \\
\hline $\mathrm{Li}_{2} \mathrm{O}$ & $\begin{array}{l}\text { Low } \\
\text { High }\end{array}$ & (a) & $\begin{array}{l}.1584^{\left({ }^{(}\right)} \\
.2007^{(\mathrm{c})} \\
\end{array}$ & $\begin{array}{l}.1697^{\left({ }^{(}\right)} \\
.2493^{\left({ }^{\circ}\right)}\end{array}$ & $\begin{array}{l}.0572 \\
.1284 \\
\end{array}$ & $\begin{array}{l}.0000 \\
.1145 \\
\end{array}$ & $\begin{array}{l}.0576 \\
.1076 \\
\end{array}$ & $\begin{array}{l}.0587 \\
.1076 \\
\end{array}$ \\
\hline $\mathrm{MgO}$ & $\begin{array}{l}\text { Low } \\
\text { High }\end{array}$ & (a) & $\begin{array}{l}.0152^{(\mathrm{c})} \\
.0446^{(\mathrm{c})} \\
\end{array}$ & $\begin{array}{l}.0082^{\left({ }^{(}\right)} \\
.0418^{\left({ }^{(c)}\right.} \\
\end{array}$ & (b) & $\begin{array}{l}.0000 \\
.0508 \\
\end{array}$ & $\begin{array}{l}.0084 \\
.0508 \\
\end{array}$ & $\begin{array}{l}.0084 \\
.0268 \\
\end{array}$ \\
\hline $\mathrm{MnO}$ & $\begin{array}{l}\text { Low } \\
\text { High }\end{array}$ & (a) & $\begin{array}{l}.0040 \\
.0215 \\
\end{array}$ & $\begin{array}{l}.0009 \\
.0170 \\
\end{array}$ & (b) & $\begin{array}{l}.0000 \\
.0322 \\
\end{array}$ & $\begin{array}{l}.0000 \\
.0322 \\
\end{array}$ & $\begin{array}{l}.0119 \\
.0322 \\
\end{array}$ \\
\hline $\mathrm{Na}_{2} \mathrm{O}$ & $\begin{array}{l}\text { Low } \\
\text { High }\end{array}$ & $\begin{array}{l}.1344 \\
.273 \\
\end{array}$ & $\begin{array}{l}.1584^{\left({ }^{(}\right)} \\
.2007^{(\mathrm{c})} \\
\end{array}$ & $\begin{array}{l}.1697^{(0)} \\
.2493^{\left({ }^{\circ}\right)} \\
\end{array}$ & $\begin{array}{l}.0646 \\
.1342 \\
\end{array}$ & $\begin{array}{l}.0660 \\
.2576 \\
\end{array}$ & $\begin{array}{l}.0691 \\
.1702 \\
\end{array}$ & $\begin{array}{l}.0691 \\
.1702 \\
\end{array}$ \\
\hline $\mathrm{NiO}$ & $\begin{array}{l}\text { Low } \\
\text { High }\end{array}$ & (a) & (b) & (b) & (b) & $\begin{array}{l}.0000 \\
.0256 \\
\end{array}$ & $\begin{array}{l}.0035 \\
.0256 \\
\end{array}$ & $\begin{array}{l}.0035 \\
.0147 \\
\end{array}$ \\
\hline $\mathrm{P}_{2} \mathrm{O}_{5}$ & $\begin{array}{l}\text { Low } \\
\text { High }\end{array}$ & (a) & $\begin{array}{l}.0063 \\
.0181 \\
\end{array}$ & $\begin{array}{l}.0003 \\
.0225 \\
\end{array}$ & (b) & (b) & (b) & (b) \\
\hline $\mathrm{SiO}_{2}$ & $\begin{array}{l}\text { Low } \\
\text { High }\end{array}$ & $\begin{array}{l}.4551 \\
.6049 \\
\end{array}$ & $\begin{array}{l}.4520 \\
.5382 \\
\end{array}$ & $\begin{array}{l}.4555 \\
.5432 \\
\end{array}$ & $\begin{array}{l}.4116 \\
.5928 \\
\end{array}$ & $\begin{array}{l}.4348 \\
.7754 \\
\end{array}$ & $\begin{array}{l}.4348 \\
.6000 \\
\end{array}$ & $\begin{array}{r}.4348 \\
.5957\end{array}$ \\
\hline $\mathrm{ThO}_{2}$ & $\begin{array}{l}\text { Low } \\
\text { High }\end{array}$ & (a) & $\begin{array}{l}.0044 \\
.0152 \\
\end{array}$ & $\begin{array}{l}.0050 \\
.0167 \\
\end{array}$ & $\begin{array}{l}.0024 \\
.0164 \\
\end{array}$ & (a) & (a) & (a) \\
\hline $\mathrm{TiO}_{2}$ & $\begin{array}{l}\text { Low } \\
\text { High }\end{array}$ & (a) & (b) & (b) & (b) & $\begin{array}{l}.0000 \\
.0271 \\
\end{array}$ & $\begin{array}{l}.0000 \\
.0148 \\
\end{array}$ & $\begin{array}{l}.0000 \\
.0141 \\
\end{array}$ \\
\hline $\mathrm{U}_{3} \mathrm{O}_{8}$ & $\begin{array}{l}\text { Low } \\
\text { High }\end{array}$ & (a) & $\begin{array}{l}.0001 \\
.0009 \\
\end{array}$ & $\begin{array}{l}.0001 \\
.0022 \\
\end{array}$ & (b) & (a) & $\cdot$ (a) & (a) \\
\hline $\mathrm{ZrO}_{2}$ & $\begin{array}{l}\text { Low } \\
\text { High }\end{array}$ & (a) & (b) & (b) & $\begin{array}{l}.0012 \\
.0122 \\
\end{array}$ & (b) & (b) & (b) \\
\hline Others & $\begin{array}{l}\text { Low } \\
\text { High }\end{array}$ & (a) & $\begin{array}{l}.0101 \\
.0386 \\
\end{array}$ & $\begin{array}{l}.0086 \\
.0555 \\
\end{array}$ & n.a. & $\begin{array}{l}.0000 \\
.0590 \\
\end{array}$ & $\begin{array}{l}.0000 \\
.0086 \\
\end{array}$ & $\begin{array}{l}.0000 \\
.0086 \\
\end{array}$ \\
\hline $\begin{array}{l}\text { PCT B } \\
\left(g / \mathrm{m}^{2}\right)\end{array}$ & $\begin{array}{l}\text { Low } \\
\text { High }\end{array}$ & $\begin{array}{r}0.075 \\
38.709 \\
\end{array}$ & $\begin{array}{l}0.160 \\
0.309 \\
\end{array}$ & $\begin{array}{l}0.179 \\
0.417 \\
\end{array}$ & $\begin{array}{r}0.186 \\
10.278 \\
\end{array}$ & $\begin{array}{r}0.055 \\
35.120 \\
\end{array}$ & $\begin{array}{l}0.175 \\
-9.250 \\
\end{array}$ & $\begin{array}{l}0.175 \\
9.250 \\
\end{array}$ \\
\hline
\end{tabular}

(a) Component not included or determined for glasses in this data set.

(b) Component included in Others for this data set.

(c) The ranges listed for $\mathrm{BaO}, \mathrm{CaO}$, and $\mathrm{MgO}$ are for $\mathrm{BaO}+\mathrm{CaO}+\mathrm{MgO}$. The ranges listed for $\mathrm{Na}_{2} \mathrm{O}, \mathrm{Li}_{2} \mathrm{O}$, and $\mathrm{K}_{2} \mathrm{O}$ are for $\mathrm{Na}_{2} \mathrm{O}+\mathrm{Li}_{2} \mathrm{O}+\mathrm{K}_{2} \mathrm{O}$. 


\subsection{CVS Data Set}

The first five phases of the Composition Variation Study (CVS) performed at the Pacific Northwest Laboratory from 1989 to 1994 studied 147 simulated waste glass compositions ${ }^{3}$. The CVS primarily varied the 10 components $\mathrm{SiO}_{2}, \mathrm{~B}_{2} \mathrm{O}_{3}, \mathrm{Al}_{2} \mathrm{O}_{3}, \mathrm{Fe}_{2} \mathrm{O}_{3}, \mathrm{ZrO}_{2}, \mathrm{Na}_{2} \mathrm{O}, \mathrm{Li}_{2} \mathrm{O}, \mathrm{CaO}$, $\mathrm{MgO}$, and Others (all remaining waste components treated as a single component), although the composition of the Others mix was varied in several glasses. The $147 \mathrm{CVS}$ glasses were studied in five phases (named CVS-I, and CVS-II Phases 1, 2, 3, and 4). The majority of the 147 glasses were selected using statistical optimal design software so as to cover the boundary and interior of an explicitly-defined composition experimental region. CVS-I, part of CVS-II Phase 2, and CVSII Phase 3 focused mainly on the boundary of the composition region. CVS-II Phase 1, part of CVS-II Phase 2, and CVS-II Phase 4 focused mainly on the interior of the composition region. CVS-II Phase 4 consisted of one-at-a-time variations of $\mathrm{SiO}_{2}, \mathrm{~B}_{2} \mathrm{O}_{3}, \mathrm{Na}_{2} \mathrm{O}, \mathrm{Li}_{2} \mathrm{O}, \mathrm{CaO}, \mathrm{MgO}$, and $\mathrm{Al}_{2} \mathrm{O}_{3}$ while keeping all remaining components in the same relative proportions as in a base composition near the center of the composition region. A detailed discussion of the experimental design of the CVS phases is contained in the report by Hrma, Piepel, et al. ${ }^{3}$

In this report, the five CVS phases are treated as five separate data sets and one combined data set. The mole fraction compositions and PCT normalized B, Li, and Na releases for the 147 CVS-I and CVS-II Phase 1-4 glasses are given in Tables A.1 to A.5 of Appendix A. The normalized releases are averages of duplicate PCT results. There are 14 replicate compositions (same compositions with different glass names) scattered throughout the five CVS phases, and it is important to realize that neither modeling approach (mixture or $\mathrm{FEH}$ ) can account for experimental variability in $\ln (\mathrm{NR})$ values among replicates. Hence, $\mathrm{R}^{2}$ values (defined in Section 5 and reported in Section 6 ) for the mixture and FEH models will be lower than they would be without replicates in the data set.

Although B, Li, and Na normalized releases are given in Tables A.1 to A.5, only B normalized releases are used in this report. The range of B normalized release values $\left(\mathrm{g} / \mathrm{m}^{2}\right)$ are: 0.066 to 20.639 for CVS-I, 0.128 to 2.937 for CVS-II Phase $1,0.173$ to 12.701 for CVS-II Phase 2, 0.115 to 44.000 for CVS-II Phase 3, 0.193 to 14.400 for CVS-II Phase 4, and 0.066 to 44.000 for the combined CVS data set [denoted CVS(all)].

Figures B.1 to B.5 in Appendix B contain scatterplot matrices for the glass compositions in each of the five CVS phases, while Figure B.6 contains the scatterplot matrix for the compositions from all five CVS phases combined. Figures B.1 to B.3 show that CVS-I, CVS-II Phase 1, and CVS-II Phase 2 glasses cover their respective experimental regions quite well. Similarly, Figure B.6 shows that the combined data set covers the overall experimental region very well. This is expected, since statistical experimental design methods and software were employed to achieve good coverage of various experimental subregions and the overall experimental region. Some pairwise scatterplots in Figures B.1 to B.4 and B.6 do not have any points in the upper right hand corners, but this is due to using multicomponent constraints to exclude such compositions from study. Figures B. 2 to B. 4 and B. 6 show only single points with the lowest $\mathrm{SiO}_{2}$ level, but these compositions were specially selected outside the specified experimental region. Within the experimental region, the $\mathrm{SiO}_{2}$ range is covered very well. Figure B.3 shows that CVS-II Phase 3 
provides a good coverage of the experimental region relative to most pairs of components, but a somewhat uneven coverage for a few pairs. This is expected, since CVS-II Phase 3 compositions were selected to augment the data available through the completion of CVS-II Phase 2. Also, compositions with extreme glass property values were selected for CVS-II Phase 3. These goals for selecting points led to avoiding portions of composition space covered in previous CVS phases and focusing on undercovered portions, which caused a somewhat uneven coverage of composition space in CVS-II Phase 3. CVS-II Phase 4 varied selected components one-at-a-time while keeping all remaining components in the same relative proportions. This proportional adjustment leads to most points in Figure B.5 scatterplots lying along straight lines, and these lines are also visible in Figure B. 6 for the combined data set. In summary, the data for each of the five CVS experimental phases (with the possible exception of CVS-II Phase 4), and the combined CVS data set, should provide good bases for fitting and comparing FOM and FEH models.

\subsection{Ramsey Data Set}

A paper by Öksoy, Pye, Bickford, and Ramsey ${ }^{6}$ contains mole fraction compositions and unnormalized PCT releases for 30 glasses. The glasses are from a statistically-designed study of a seven-component glass composition region investigated by W.G. Ramsey [a Savannah River Technology Center (SRTC) staff member] as part of his Ph.D. research at Clemson University. Hence, the data set is referred to as the Ramsey data set in this report. Whereas most of the data sets used in this report are for glass systems with a large number of components, the Ramsey data set is different in that it is for a smaller system containing only seven components $\left(\mathrm{Al}_{2} \mathrm{O}_{3}, \mathrm{~B}_{2} \mathrm{O}_{3}\right.$, $\mathrm{CaO}, \mathrm{Fe}_{2} \mathrm{O}_{3}, \mathrm{FeO}, \mathrm{Na}_{2} \mathrm{O}$, and $\mathrm{SiO}_{2}$ ).

The mole fraction compositions and PCT normalized $\mathrm{Si}, \mathrm{B}$, and $\mathrm{Na}$ releases for the 30 Ramsey glasses are listed in Table A.6 of Appendix A. The normalized releases were calculated by first averaging unnormalized releases from triplicate PCTs, and then normalizing the averages. Only the B normalized releases are used in this report. The range of B normalized release values for the Ramsey data set is 0.075 to $38.709 \mathrm{~g} / \mathrm{m}^{2}$.

The scatterplot matrix in Figure B.7 of Appendix B shows that the Ramsey data set does a good job of covering its experimental region and that there are no strong correlations among pairs of components, with one exception. $\mathrm{Fe}_{2} \mathrm{O}_{3}$ and $\mathrm{FeO}$ exhibit a fairly strong positive correlation. This could make it difficult for mixture models to properly separate the effects of these two variables on PCT durability.

\subsection{West Valley 1987 and 1988 Data Sets}

Pacific Northwest Laboratory (PNL) performed two composition variation studies for West Valley Nuclear Services in 1987 and 1988. Each of these studies investigated 16 glasses in different composition regions defined in terms of the 11 components $\mathrm{Al}_{2} \mathrm{O}_{3}, \mathrm{~B}_{2} \mathrm{O}_{3}$, $\mathrm{BaO}+\mathrm{CaO}+\mathrm{MgO}, \mathrm{Fe}_{2} \mathrm{O}_{3}, \mathrm{~K}_{2} \mathrm{O}+\mathrm{Li}_{2} \mathrm{O}+\mathrm{Na}_{2} \mathrm{O}, \mathrm{MnO}_{2}, \mathrm{P}_{2} \mathrm{O}_{5}, \mathrm{SiO}_{2}, \mathrm{ThO}_{2}, \mathrm{UO}_{2}$, and Others (all remaining components). (Note that $\mathrm{UO}_{2}$ was converted to $\mathrm{U}_{3} \mathrm{O}_{8}$ for the modeling work in this report to be consistent with Jantzen et al. ${ }^{5}$ ) In each of the 1987 and 1988 studies, 15 of the 16 glasses were selected by statistical methods to evenly cover the boundary of the experimental 
region. A sixteenth composition from the center of each region was included in each of the experimental designs ${ }^{7}$. PNL tested these glasses with the PCT in 1991 and 1992, and the results were ultimately published in a 1994 report $^{8}$.

The mole fraction compositions and PCT averaged normalized $\mathrm{B}, \mathrm{Li}$, and $\mathrm{Na}$ releases for the WV87 and WV88 data.sets are given in Tables A.7 and A.8 of Appendix A. Only B normalized releases are used in this report. The range of B normalized release values for the WV87 data set is 0.160 to $0.309 \mathrm{~g} / \mathrm{m}^{2}$, and for the WV88 data set is 0.179 to $0.417 \mathrm{~g} / \mathrm{m}^{2}$.

Scatterplot matrices for the WV87 and WV88 data sets are given in Figures B.8 and B.9 of Appendix B. Many of the pairwise plots in each figure reflect that the designs consist of 15 vertices (boundary points) and one center point of the respective experimental regions. Some pairwise plots show components having values intermediate in their ranges, but this is possible with vertices of polyhedral experimental regions. Strong correlations are seen in the plots corresponding to pairs of components that were grouped, i.e., $\mathrm{BaO}+\mathrm{CaO}+\mathrm{MgO}$ and $\mathrm{K}_{2} \mathrm{O}+\mathrm{Li}_{2} \mathrm{O}+\mathrm{Na}_{2} \mathrm{O}$. However, these strong correlations are of no consequence as long as the grouped components are treated as a single component in mixture modeling efforts. Overall, each data set covers its experimental region well, in keeping with the statistical experimental design methods used to select the compositions?

\subsection{WVDP Data Set}

West Valley Nuclear Services (WVNS) developed a data set consisting of 58 glass compositions and PCT elemental releases, and used this data set to develop PCT release models ${ }^{\circ}$ for use in the West Valley Demonstration Plant (WVDP), a high-level nuclear waste glass facility. The 58 glasses were selected to evenly cover a glass composition region centered on the WVDP target glass composition with boundary taken to be three times the expected process variation. Although the WVDP target glass composition contains a large number of components, variations in many of them are not expected to significantly affect PCT releases. Thus, with the exception of three glasses, only nine components $\left(\mathrm{SiO}_{2}, \mathrm{Al}_{2} \mathrm{O}_{3}, \mathrm{ZrO}_{2}, \mathrm{ThO}_{2}, \mathrm{Na}_{2} \mathrm{O}, \mathrm{K}_{2} \mathrm{O}, \mathrm{Li}_{2} \mathrm{O}, \mathrm{B}_{2} \mathrm{O}_{3}, \mathrm{Fe}_{2} \mathrm{O}_{3}\right)$ were varied in the 58 WVDP glass compositions. This nature of the data set does not support treating the remaining components as a combined Others component as was done in the CVS (Section 4.1), because Others is necessarily also constant except for the three glasses. The variation in Others for the three glasses is small, hence the WVDP data set does not support the use of an Others component. For purposes of fitting mixture models, the compositions of the nine components (listed above) were normalized to a total mole fraction of 1.0 .

The mole fraction compositions and PCT averaged normalized $\mathrm{B}, \mathrm{Li}$, and $\mathrm{Na}$ releases for the 58 WVDP glasses are given in Table A.9 of Appendix A. Only B normalized releases are used in this report. The range of $B$ normalized release values for the WVDP data set is 0.186 to 10.278 $\mathrm{g} / \mathrm{m}^{2}$.

Composition \#51 in Table A.9 was determined to be an outlier by WVNS and was not used in developing their PCT models. Regression diagnostics obtained in fitting the FOM model to all 
58 data points confirmed that \#51 was an outlier, and so it was not used for fitting mixture or FEH models in this report.

The scatterplot matrix for the WVDP data set (without observation \#51) is given in Figure B.10 of Appendix B. The scatterplot matrix used the compositions normalized to a total mole fraction of 1.0 for the nine components shown in the plot. The scatterplot matrix shows that the WVDP data generally cover the underlying composition region quite well. The pairwise plots of $\mathrm{Na}_{2} \mathrm{O}, \mathrm{K}_{2} \mathrm{O}$, and $\mathrm{Li}_{2} \mathrm{O}$ show positive correlations for subsets of the data, but there are sufficient additional data that this should not be a problem. The pairwise plots involving $\mathrm{ZrO}_{2}$ show some patterns for small subsets of the data, but this likewise should not be a problem. However, the small mole fraction ranges of $\mathrm{ZrO}_{2}$ and $\mathrm{ThO}_{2}$ limit assessing component effects and making model predictions to the small ranges of these components.

\subsection{SRTC/DWPF Homogenous Glasses Data Set}

The Savannah River Technology Center (SRTC) tested the PCT durability of many glasses and developed PCT normalized release prediction models for the Defense Waste Processing Facility (DWPF) high-level nuclear waste glass plant ${ }^{5}$. SRTC separated their glasses into homogeneous and inhomogeneous sets. Glasses were classified as homogeneous if they contain no crystalline species and are not liquid-liquid phase separated. Glasses with crystallinity or phase separation were classified as inhomogeneous. PCT normalized releases are often (but not always) higher for inhomogeneous glasses than for homogeneous glasses, due to higher solubility in water of one of the phases. Because of this, Jantzen et al. ${ }^{5}$ modeled PCT normalized releases of homogeneous and inhomogeneous glasses separately. Only the homogeneous data set is modeled here, because the inhomogeneous data set contains too few data points and has other limitations.

The SRTC homogeneous data set (as defined in early 1995) contains 137 glasses, with triplicate PCT tests performed on each (with a few exceptions). Of the 137 glasses, 43 are replicates (i.e., the same glasses tested by the PCT at different times). The averages of the triplicate PCT releases for each of the 137 glasses are used for modeling in this report. The triplicate PCT releases for each of the 137 glasses only contain short-term testing and analytical sources of variation, whereas the replicate glass releases contain longer-term sources of variation. Using averaged triplicate PCT releases for modeling avoids improperly diluting the experimental error variance estimate obtained (from the replicate glasses) in least squares regression. Note that $\mathrm{R}^{2}$ values (see Section 5) for mixture and FEH models fitted to averages of triplicate PCT releases will be somewhat larger than if unaveraged triplicate results were used. This is because models cannot account for the experimental variation represented by triplicate PCT tests. However, even - using averages of triplicate PCT results, $R^{2}$ values for this data will be lower than they would be if the 137 glasses did not include replicates. This should not be viewed negatively, because having replicates in a data set permits statistically testing fitted models for lack-of-fit. Statistical lack-offit tests are not considered in this report, but the interested reader should see Section 1.5 of Draper and Smith ${ }^{10}$.

The mole fraction compositions and PCT averaged normalized $\mathrm{B}, \mathrm{Li}$, and $\mathrm{Na}$ releases for the 137 SRTC homogeneous glasses can be found in Table A.10 of Appendix A. Only the B 
normalized releases are used in this report. The range of $B$ normalized release values for the full SRTC homogeneous data set is 0.055 to $35.12 \mathrm{~g} / \mathrm{m}^{2}$. The range without the two sets of outlying compositions (as discussed in following paragraphs) is 0.175 to $9.25 \mathrm{~g} / \mathrm{m}^{2}$.

The scatterplot matrix for the 137-point SRTC homogeneous data set is given in Figure B.11 in Appendix B. The scatterplot matrix shows considerable clustering of the glass compositions, uncovered portions of glass composition space, and a few glasses with outlying values of several components (e.g., $\mathrm{CaO}, \mathrm{Li}_{2} \mathrm{O}, \mathrm{Na}_{2} \mathrm{O}, \mathrm{SiO}_{2}$, and Others). Of particular note is the $\mathrm{CaO}$ vs. $\mathrm{Na}_{2} \mathrm{O}$ scatterplot, which shows a very strong positive correlation between these components due to a few compositions. The NBS623 glass, the 14 replicates of the ARM1 glass, and the series of four MG glasses (MG-9, MG-18, MG-25, and MG-28) were identified as being the outlying compositions. These compositions are very atypical of the other glasses in the data set, and of waste glasses expected to be produced by the DWPF. In fact, several components (not shown in Figure B.11) varied significantly over the data set only because of these few glasses. Such atypical, outlying compositions can have a major impact when models are fitted to the data.

Figure B.12 contains the scatterplot matrix without the 19 atypical NBS623, ARM1, and MG compositions (118 data points). Considerable clustering of compositions, uncovered or lightly-covered portions of composition space, and outlying component values are still evident in Figure B.12. Outlying compositions of particular note are the composition with a low $\mathrm{SiO}_{2}$ value, the two compositions with larger $\mathrm{MgO}$ values, and the five compositions that have larger values of both $\mathrm{NiO}$ and $\mathrm{FeO}$ than the remaining glasses. The $\mathrm{FeO}$ vs. $\mathrm{NiO}$ scatterplot shows a very strong correlation between $\mathrm{FeO}$ and $\mathrm{NiO}$, indicating that the data do not provide adequate support for separately determining the effects of $\mathrm{FeO}$ and $\mathrm{NiO}$ on durability.

In Section 6, FEH and FOM models are fitted to both the whole SRTC homogeneous data set (referred to as SRTC 137), and also the subset of data obtained by excluding the 19 atypical data points discussed above (referred to as SRTC 118). The scatterplot matrix in Figure B.11 indicates that extreme caution should be used regarding models developed from the whole data set. The scatterplot matrix in Figure B.12 after deleting the 19 atypical data points looks better, but still the data does not cover the composition space as well as might be desired. This could have undesirable effects on models developed using the SRTC 118 data. Hence, in Section 6 a FOM model is also fit to a data set obtained by deleting the two compositions with larger $\mathrm{MgO}$ values, and the five compositions with larger values of $\mathrm{NiO}$ and $\mathrm{FeO}$ (referred to as SRTC 111). A scatterplot matrix of the SRTC 111 composition data is given in Figure B.13. 


\section{Statistics Used to Compare Models}

This section describes the goodness-of-fit statistics $R^{2}, R^{2}(A D J), R^{2}(P R E S S)$, and $s$ used to assess and compare the mixture and FEH models fitted to each of the data sets described in Section 4. Descriptions and formulas for these statistics are given below. The notation used in the formulas is defined following the formulas.

$\mathrm{R}^{2} \quad$ The fraction of variability in the $\ln (\mathrm{NR})$ data accounted for by a fitted model.

$$
R^{2}=1-\frac{\sum_{i=1}^{n}\left(y_{i}-\hat{y}_{i}\right)^{2}}{\sum_{i=1}^{n}\left(y_{i}-\bar{y}\right)^{2}}
$$

$\mathrm{R}^{2}(\mathrm{ADJ}) \quad$ The fraction of variability in the $\ln (\mathrm{NR})$ data accounted for by the fitted model, adjusted for the number of coefficients and number of data points used in fitting the model. $R^{2}(A D J)$ is also useful for comparing fitted models based on different numbers of coefficients.

$$
R^{2}(A D J)=1-\frac{\sum_{i=1}^{n}\left(y_{i}-\hat{y}_{i}\right)^{2} /(n-p)}{\sum_{i=1}^{n}\left(y_{i}-\bar{y}\right)^{2} /(n-1)}
$$

$\mathrm{R}^{2}$ (PRESS) The fraction of variability in the $\ln (\mathrm{NR})$ data accounted for by the fitted model, where each data point is "left out of the fit" in evaluating how well the model predicts the property for that data point. $\mathrm{R}^{2}$ (PRESS) estimates the fraction of variability that would be explained in predicting new observations drawn from the same composition space.

$$
R^{2}(\text { PRESS })=1-\frac{\sum_{i=1}^{n}\left(y_{i}-\hat{y}_{(i)}\right)^{2}}{\sum_{i=1}^{n}\left(y_{i}-\bar{y}\right)^{2}}
$$

The square root of the mean squared error of the fitted model, which is a measure of the variability of model prediction errors.

$$
s=\sqrt{\frac{\sum_{i=1}^{n}\left(y_{i}-\hat{y}_{i}\right)^{2}}{n-p}}
$$


In the preceding definitions,

$\mathrm{n}=$ the number of data points used to fit the model,

$\mathrm{p}=$ the number of fitted coefficients in the model,

$y_{i}=$ the measured $\ln (N R)$ value for the $i^{\text {th }}$ data point,

$\hat{y}_{i}=$ the predicted $\ln (\mathrm{NR})$ value for the $\mathrm{i}^{\text {th }}$ data point made using the model fitted to all $\mathrm{n}$ data points,

$\hat{y}_{(i)}=$ the predicted $\ln (\mathrm{NR})$ value for the $\mathrm{i}^{\text {th }}$ data point made using a model fitted to all $n$ data points except the $\mathrm{i}^{\text {th }}$,

$\overline{\mathrm{y}}=$ the average of the $\mathrm{n}$ measured $\ln (\mathrm{NR})$ values $\mathrm{y}_{\mathrm{i}}$.

The numerator of the ratio in (14) is referred to as the PRESS statistic, where PRESS is an acronym that stands for PRedicted Error Sums of Squares. $R^{2}$ (PRESS) is also referred to as cross-validation $R^{2}$ in the literature.

The $\mathrm{R}^{2}$ statistic must be between 0 and 1 for models fitted to a data set by least squares. Generally, $R^{2}$ (ADJ) and $R^{2}$ (PRESS) also are between 0 and 1 , but they can be negative for a poor-fitting model, a model that contains many more coefficients than needed to fit the data, or a model fitted to data with one or more very influential data points. The maximum possible values of the three $R^{2}$ statistics will be less than 1 for a data set that contains replicate compositions whose $y_{i}=\ln (\mathrm{NR})$ values are different due to experimental variation, since a model relating $\ln (\mathrm{NR})$ to composition cannot account for such experimental variation. Among the three $\mathrm{R}^{2}$ statistics, $R^{2}>R^{2}(A D J)$, and typically $R^{2}(A D J)>R^{2}$ (PRESS). More than a minor difference between $R^{2}$ and $R^{2}(A D J)$ indicates that the model may contain more coefficients than needed. $A$ substantial difference between $R^{2}$ and $R^{2}$ (PRESS) indicates that one or more data points are very influential in determining the fit of the model. Some reduction from $R^{2}$ to $R^{2}$ (PRESS) is expected because $R^{2}$ corresponds to using all data to fit the model, whereas $R^{2}$ (PRESS) corresponds to leaving each data point out of the fit when evaluating the performance of the model for that point. In general, a model will tend to predict better for data used to fit it than for data not used to fit it. Technically, $\mathrm{R}^{2}$ (PRESS) evaluates model performance using data not used in fitting the model, so it can be considered a "model validation" rather than a "model evaluation" technique.

The s statistic has the same units as $\ln (\mathrm{NR})\left[\ln \left(\mathrm{g} / \mathrm{m}^{2}\right)\right.$ in this report $]$, and estimates the experimental standard deviation when the corresponding model is appropriate (i.e., does not have a statistically significant lack-of-fit) for the data set. When a model is not appropriate (i.e., does have a statistically significant lack-of-fit), the $s$ statistic will be larger than the experimental standard deviation. Experience with the PCT on high-level waste glasses indicates that the experimental relative standard deviation (RSD) for single PCT determinations (i.e., a single PCT and chemical analysis) conducted on the same glass at different times is approximately $0.06-0.14$ (see Table F.5 in Appendix F of the report by Hrma, Piepel, et al. ${ }^{3}$ ). The work of Hrma, Piepel, et al. ${ }^{3}$ (Table F.5) also shows that the RSD remains in the same $0.06-0.14$ range for an average of two determinations conducted at the same time. Based on that work, it can be concluded that the RSD would be in the same $0.06-0.14$ range for an average of three determinations conducted 
at the same time. The RSDs do not decrease much because only short-term variation in PCT results is being averaged. Then, according to the relationship in (4), the experimental standard deviation [in $\ln (\mathrm{NR})$ units] is approximately equal to the experimental RSD (which is unitless). Hence, the experimental standard deviation in $\ln (\mathrm{NR})$ units is in the range $0.06-0.14$ regardless of whether a single determination, an average of two same-time determinations, or an average of three same-time determinations is used to represent the PCT release of a glass. The s statistic for a fitted model can be compared to this $0.06-0.14$ range to subjectively assess whether the fitted model may have a statistically significant lack-of-fit. Objective statistical tests for model lack-offit exist (see Section 1.5 of Draper and $S \mathrm{Smith}^{10}$ ), but are not considered or applied in this report. 



\section{Mixture and Free Energy of Hydration Models Fitted to Data Sets}

The results of fitting the FEH and FOM models to each of the data sets described in Section 4 are presented in this section. Results from fitting SOM models to three of the data sets are also presented. The results consist of the fitted model coefficients (obtained by unweighted least squares regression) and the goodness-of-fit statistics $R^{2}, R^{2}(A D J), R^{2}(P R E S S)$, and $s$ described in Section 5. The results are compared and discussed in Section 7.

Table 3 contains the results of fitting the FEH model (3) to each of the data sets described in Section 4. Included in Table 3 are the $a$ and $b$ coefficients and their standard deviations obtained by least squares regression, as well as the $R^{2}, R^{2}(A D J), R^{2}(P R E S S)$, and $s$ statistics.

Table 4 contains the results of fitting the FOM model (1) to each of the data sets described in Section 4 . Included are the $b_{i}$ coefficients and their standard deviations obtained by least squares regression, as well as the $R^{2}, R^{2}(A D J), R^{2}$ (PRESS), and $s$ statistics. Also included in Table 4 for each data set are the $c_{i}$ and $d_{i}$ coefficients obtained via (5d) from the FEH fitted model for that data set along with the corresponding standard deviations of the coefficients. For each data set, the $c_{i}$ and $d_{i}$ coefficients calculated from the FEH model can be directly compared to the $b_{i}$ coefficients from the FOM model. The standard deviations of the coefficients for a given component can be used to ascertain whether there is a significant difference between the coefficients for that component.

Ranges of coefficients and standard deviations for the Others component in the FEH model (converted to the form of a FOM model) are also included in Table 4. A single Others coefficient and standard deviation exists only if the "Others mix" is fixed for all glasses in a data set, as is the case for the CVS-I data set. However, the remaining data sets have different "Others mixes" for different glasses in the data sets. In such cases, an Others component coefficient was computed for each glass as a weighted average of the coefficients for each component making up Others, with the relative mole fractions of components in Others used as weights. Also, the coefficient for each component making up Others was computed per (5d) for each data set, with the results given in Table 5. A standard deviation was computed for each Others component coefficient for each glass. The ranges of Others coefficients and standard deviations obtained across all the glasses in each data set are summarized in Table 4.

Reduced SOM models (i.e., SOM models containing only some of the second-order terms) were fitted to the WVDP, CVS(all), and Ramsey data sets. The resulting fitted models and goodness-of-fit statistics are given in Table 6. Stepwise regression techniques ${ }^{10}$ were used to add statistically significant second-order terms (squares or crossproducts of oxide component mole fractions) to FOM models.

The reduced SOM model given in Table 6 for the Ramsey data was obtained via stepwise regression using a very conservative stopping criterion. Stepwise regression with a less conservative stopping criterion, as well as all-subsets regression ${ }^{10}$ (in which all possible combinations of second-order terms taken two, three, four, .... at-a-time are added to the firstorder mixture model), suggested that adding up to six second-order terms may improve the model 
fit. However, several models of each size (number of second-order terms added) have nearly identical $R^{2}$ values, and thus it was difficult to pick just one of them for inclusion in Table 6. To make this point clearer, the following chart shows the $\mathrm{R}^{2}$ values for five options of adding five second-order terms to the first-order mixture model:

\begin{tabular}{|c|c|}
\hline $\begin{array}{l}\text { Total Number } \\
\text { Model Terms }\end{array}$ & $\mathrm{R}^{2}$ \\
\hline 12 & 0.97634665 \\
\hline 12 & 0.97440217 \\
\hline 12 & 0.97418499 \\
\hline 12 & 0.97415623 \\
\hline 12 & 0.97325151 \\
\hline
\end{tabular}

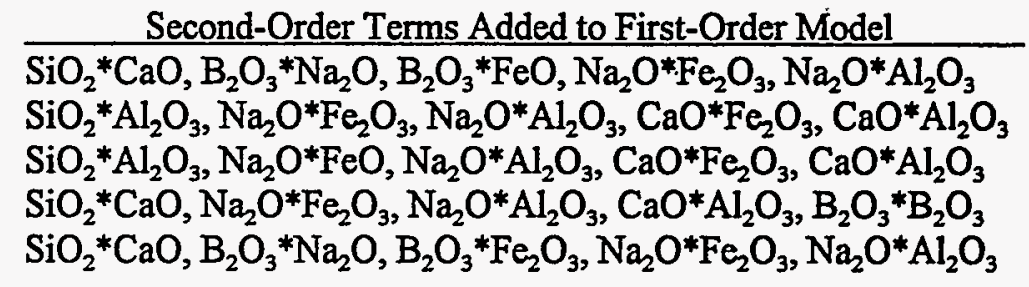

Care must be taken regarding the interpretation of second-order terms in reduced SOM models. The second-order terms selected for the reduced SOM models are those that most improved the model fit for each data set, and are not necessarily interpretable or explainable from a glass science perspective. Squared and crossproduct terms are always correlated with each other and with the first-order terms. The magnitudes of these correlations are influenced by the magnitudes of correlations among the glass components for each data set. These correlations can cause stepwise regression to select second-order terms that may or may not directly represent the true underlying curvilinear or interactive behavior of components. The chart above for the Ramsey data set shows how several possible sets of second-order terms can yield similar goodness-of-fits. Our practical experience is that some but not all second-order terms selected by stepwise regression correspond to behavior expected from glass science principles. However, even if second-order terms indirectly rather than directly account for underlying nonlinear behavior, the resulting reduced SOM models are still useful for predicting $\ln (\mathrm{NR})$ values for glasses in the same composition region as the glasses used to develop the models.

Predicted versus measured plots are given in Appendix $\mathrm{C}$ for the free energy of hydration, FOM, and reduced second-order mixture models fitted to the various data sets. Such plots are useful in assessing how well the model fits the data set used to develop it. A discussion of what to look for in such plots is given at the start of Appendix C. 
Table 3. Coefficients and Goodness-of-Fit Statistics for Free Energy of Hydration Models Fitted to $\ln$ (PCT Normalized B Release in $\mathrm{g} / \mathrm{m}^{2}$ )

\begin{tabular}{lllllll}
\hline Coefficient & CVS-I & CVS-II.I & CVS-II.2 & CVS-II.3 & CVS-II.4 & CVS (all) \\
\hline a (intercept) & -2.3584 & -2.2001 & -1.1381 & -2.7293 & -2.7236 & -2.3093 \\
sd(a) & $(0.7066)$ & $(1.0687)$ & $(0.5980)$ & $(0.4639)$ & $(0.4705)$ & $(0.2734)$ \\
$\mathrm{b}$ (slope) & -0.5047 & -0.3015 & -0.2046 & -0.4395 & -0.5302 & -0.4081 \\
$\quad$ sd(b) & $(0.1165)$ & $(0.1898)$ & $(0.0944)$ & $(0.0638)$ & $(0.0683)$ & $(0.0415)$ \\
& & & & & & \\
$\mathrm{R}^{2}$ & 0.472 & 0.129 & 0.113 & 0.537 & 0.741 & 0.400 \\
$\mathrm{R}^{2}$ (ADJ) & 0.447 & 0.078 & 0.089 & 0.525 & 0.729 & 0.396 \\
$\mathrm{R}^{2}$ (PRESS) & 0.394 & -0.316 & -0.007 & 0.496 & 0.690 & 0.384 \\
$\mathrm{~s}$ & 1.364 & 0.973 & 1.171 & 1.192 & 0.662 & 1.169 \\
\hline
\end{tabular}

\begin{tabular}{lllllll}
\hline Coefficient & RAMSEY & WV87 & WV88 & WVDP & $\begin{array}{c}\text { SRTC Homo } \\
137 \text { Obs. }\end{array}$ & $\begin{array}{c}\text { SRTC Homo. } \\
118 \text { Obs. }\end{array}$ \\
\hline a (intercept) & -3.5570 & -2.1005 & -2.2325 & -3.8233 & -4.8609 & -5.1678 \\
$\quad$ sd(a) & $(0.3791)$ & $(0.2298)$ & $(0.2565)$ & $(0.3898)$ & $(0.2073)$ & $(0.2398)$ \\
b (slope) & -0.4380 & -0.1262 & -0.1476 & -0.4600 & -0.6138 & -0.6665 \\
$\quad$ sd(b) & $(0.0504)$ & $(0.0485)$ & $(0.0390)$ & $(0.0485)$ & $(0.0294)$ & $(0.0344)$ \\
& & & & & & \\
$\mathrm{R}^{2}$ & 0.730 & 0.326 & 0.506 & 0.620 & 0.763 & 0.764 \\
$\mathrm{R}^{2}$ (ADJ) & 0.720 & 0.278 & 0.471 & 0.614 & 0.762 & 0.762 \\
$\mathrm{R}^{2}$ (PRESS) & 0.673 & 0.098 & 0.361 & 0.594 & 0.751 & 0.752 \\
$\mathbf{s}$ & 0.867 & 0.155 & 0.195 & 0.714 & 0.583 & 0.547 \\
\hline
\end{tabular}


Table 4. Coefficients, Coefficient Standard Deviations (in parentheses), and Goodness-of-Fit Statistics for First-Order Mixture Models and Rewritten Free Energy of Hydration Models for $\ln \left(\right.$ PCT Normalized B Release, $\mathrm{g} / \mathrm{m}^{2}$ )

\begin{tabular}{|c|c|c|c|c|c|c|c|c|}
\hline \multirow[b]{2}{*}{ Component } & \multicolumn{2}{|c|}{ CVS-I } & \multicolumn{2}{|c|}{ CVS-II.1 } & \multicolumn{2}{|c|}{ CVS-II.2 } & \multicolumn{2}{|c|}{ CVS-II.3 } \\
\hline & Mixture & $\overrightarrow{F E H}$ & Mixture & FEH & Mixture & $\overline{\mathrm{FEH}}$ & Mixture & FEH \\
\hline$\overline{\mathrm{Al}_{2} \mathrm{O}_{3}}$ & $\begin{array}{r}41.13 \\
(3.49) \\
\end{array}$ & $\begin{array}{r}-21.38 \\
(5.04) \\
\end{array}$ & $\begin{array}{l}-34.54 \\
(15.28) \\
\end{array}$ & $\begin{array}{r}-13.56 \\
(8.20) \\
\end{array}$ & $\begin{array}{l}-63.81 \\
(10.91)\end{array}$ & $\begin{array}{l}-8.85 \\
(4.13)\end{array}$ & $\begin{array}{r}-39.85 \\
(3.40) \\
\end{array}$ & $\begin{array}{r}-19.29 \\
(2.84)\end{array}$ \\
\hline $\mathrm{B}_{2} \mathrm{O}_{3}$ & $\begin{array}{c}9.42 \\
(1.75) \\
\end{array}$ & $\begin{array}{c}2.91 \\
(0.64) \\
\end{array}$ & $\begin{array}{r}11.59 \\
(6.87) \\
\end{array}$ & $\begin{array}{c}0.94 \\
(0.96) \\
\end{array}$ & $\begin{array}{l}19.17 \\
(3.58) \\
\end{array}$ & $\begin{array}{c}1.00 \\
(0.46) \\
\end{array}$ & $\begin{array}{l}17.48 \\
(2.53) \\
\end{array}$ & $\begin{array}{c}1.85 \\
(0.30) \\
\end{array}$ \\
\hline $\mathrm{CaO}$ & $\begin{array}{l}-3.46 \\
(2.70)\end{array}$ & $\begin{array}{c}4.61 \\
(1.00) \\
\end{array}$ & $\begin{array}{l}-5.66 \\
(5.56) \\
\end{array}$ & $\begin{array}{c}1.96 \\
(1.59) \\
\end{array}$ & $\begin{array}{r}-14.41 \\
(3.95) \\
\end{array}$ & $\begin{array}{c}1.69 \\
(0.76) \\
\end{array}$ & $\begin{array}{r}-12.76 \\
(5.14) \\
\end{array}$ & $\begin{array}{c}3.34 \\
(0.49) \\
\end{array}$ \\
\hline $\mathrm{Fe}_{2} \mathrm{O}_{3}$ & $\begin{array}{r}-14.84 \\
(5.23) \\
\end{array}$ & $\begin{array}{l}-9.71 \\
(2.36) \\
\end{array}$ & $\begin{array}{c}27.61 \\
(21.96) \\
\end{array}$ & $\begin{array}{l}-6.59 \\
(3.81)\end{array}$ & $\begin{array}{l}-13.62 \\
(10.75) \\
\end{array}$ & $\begin{array}{l}-4.12 \\
(1.95)\end{array}$ & $\begin{array}{r}-11.97 \\
(6.80) \\
\end{array}$ & $\begin{array}{l}-9.13 \\
(1.37)\end{array}$ \\
\hline $\mathrm{Li}_{2} \mathrm{O}$ & $\begin{array}{c}7.69 \\
(2.04) \\
\end{array}$ & $\begin{array}{c}9.77 \\
(2.17) \\
\end{array}$ & $\begin{array}{c}5.35 \\
(10.84) \\
\end{array}$ & $\begin{array}{r}5.05 \\
(3.52) \\
\end{array}$ & $\begin{array}{r}18.96 \\
(4.39) \\
\end{array}$ & $\begin{array}{c}3.78 \\
(1.71) \\
\end{array}$ & $\begin{array}{l}12.89 \\
(2.13) \\
\end{array}$ & $\begin{array}{r}7.84 \\
(1.12) \\
\end{array}$ \\
\hline $\mathrm{MgO}$ & $\begin{array}{c}8.92 \\
(2.08) \\
\end{array}$ & $\begin{array}{c}0.96 \\
(0.31) \\
\end{array}$ & $\begin{array}{c}-0.87 \\
(6.31) \\
\end{array}$ & $\begin{array}{l}-0.22 \\
(0.30)\end{array}$ & $\begin{array}{c}5.14 \\
(3.02)\end{array}$ & $\begin{array}{c}0.21 \\
(0.19)\end{array}$ & $\begin{array}{l}11.68 \\
(4.80)\end{array}$ & $\begin{array}{c}0.16 \\
(0.18) \\
\end{array}$ \\
\hline $\mathrm{Na}_{2} \mathrm{O}$ & $\begin{array}{l}13.16 \\
(2.56) \\
\end{array}$ & $\begin{array}{l}24.44 \\
(5.54) \\
\end{array}$ & $\begin{array}{l}12.41 \\
(9.68) \\
\end{array}$ & $\begin{array}{c}13.81 \\
(9.03) \\
\end{array}$ & $\begin{array}{r}24.49 \\
(4.03) \\
\end{array}$ & $\begin{array}{r}9.72 \\
(4.45) \\
\end{array}$ & $\begin{array}{l}19.97 \\
(1.97) \\
\end{array}$ & $\begin{array}{l}20.60 \\
(2.96) \\
\end{array}$ \\
\hline $\mathrm{SiO}_{2}$ & $\begin{array}{l}-2.01 \\
(0.88) \\
\end{array}$ & $\begin{array}{l}-4.40 \\
(1.15) \\
\end{array}$ & $\begin{array}{l}-5.59 \\
(4.25) \\
\end{array}$ & $\begin{array}{l}-3.42 \\
(1.83)\end{array}$ & $\begin{array}{l}-5.36 \\
(1.41) \\
\end{array}$ & $\begin{array}{l}-1.97 \\
(0.97)\end{array}$ & $\begin{array}{l}-5.69 \\
(1.00) \\
\end{array}$ & $\begin{array}{l}-4.51 \\
(0.71) \\
\end{array}$ \\
\hline $\mathrm{ZrO}_{2}$ & $\begin{array}{r}-17.06 \\
(4.64) \\
\end{array}$ & $\begin{array}{r}-11.19 \\
(2.70) \\
\end{array}$ & $\begin{array}{c}0.92 \\
(17.60) \\
\end{array}$ & $\begin{array}{l}-7.47 \\
(4.37) \\
\end{array}$ & $\begin{array}{l}-40.24 \\
(11.54)\end{array}$ & $\begin{array}{l}-4.72 \\
(2.23)\end{array}$ & $\begin{array}{c}-28.41 \\
(5.69)\end{array}$ & $\begin{array}{l}-10.42 \\
(1.55) \\
\end{array}$ \\
\hline $\begin{array}{l}\text { Others } \\
\text { prevalent } \\
\text { values }\end{array}$ & $\begin{array}{c}3.23^{(1)} \\
(4.71)\end{array}$ & $\begin{array}{c}0.96^{(0)} \\
(0.31)\end{array}$ & $\begin{array}{c}15.05^{(1)} \\
(13.95)\end{array}$ & $\begin{array}{l}-0.29 \text { to }-0.22^{(0)} \\
(0.27 \text { to } 0.30) \\
-0.22 \\
(0.30)\end{array}$ & $\begin{array}{l}-4.32^{(t)} \\
(7.54)\end{array}$ & $\begin{array}{l}-0.17 \text { to } 2.16^{(0)} \\
(0.19 \text { to } 0.97) \\
0.21: \\
(0.19)\end{array}$ & $\begin{array}{l}-0.78^{(\mathrm{n})} \\
(6.23)\end{array}$ & $\begin{array}{l}-3.01 \text { to } 19.95^{(6)} \\
(0.18 \text { to } 2.87) \\
0.16 \\
(0.18)\end{array}$ \\
\hline $\begin{array}{l}\mathbf{R}^{2} \\
\mathrm{R}^{2} / \mathrm{ADN}\end{array}$ & 0.954 & 0.472 & 0.794 & 0.129 & 0.694 & 0.113 & 0.911 & 0.537 \\
\hline $\mathrm{R}^{2}(\mathrm{ADJ})$ & 0.921 & 0.447 & 0.588 & 0.078 & 0.599 & 0.089 & 0.887 & 0.525 \\
\hline $\mathrm{R}^{2}$ (PRESS) & 0.880 & 0.394 & -0.281 & -0.316 & 0.310 & -0.007 & 0.848 & 0.496 \\
\hline s & 0.515 & 1.364 & 0.651 & 0.973 & 0.777 & 1.171 & 0.582 & 1.192 \\
\hline
\end{tabular}

(a) The Others coefficient in the first-order mixture model may be considered to be an average coefficient for all the components contained in Others.

(b) Others coefficients for FEH models were computed by forming a weighted average of the coefficients for the individual Others components, where the relative propoitions of the Others components were used as weights. This can yield different weighted averages across the data points in the data set, so the ranges of coefficient values and standard deviations are reported. There is no range for CVS-I glasses, since the relative proportions of components in Others are the same for each of these glasses. For CVS-II.1, CVS II.2, and CVS II.3, the majority of glasses have the same relative proportions in Others as in CVS-I, and these "prevalent" coefficients and standard deviations are also shown. 
Table 4. Coefficients, Coefficient Standard Deviations (in parentheses), and Goodness-of-Fit Statistics for First-Order Mixture Models and Rewritten Free Energy of Hydration Models for $\ln \left(\right.$ PCT Normalized B Release, $\mathrm{g} / \mathrm{m}^{2}$ ) (continued)

\begin{tabular}{|c|c|c|c|c|c|c|}
\hline \multirow[b]{2}{*}{ Component } & \multicolumn{2}{|c|}{ CVS-II.4 } & \multicolumn{2}{|c|}{ CVS(all) } & \multicolumn{2}{|c|}{ RAMSEY } \\
\hline & Mixture & FEH & Mixture & FEH & Mixture & FEH \\
\hline $\mathrm{Al}_{2} \mathrm{O}_{3}$ & $\begin{array}{l}-32.63 \\
(4.94)\end{array}$ & $\begin{array}{c}-22.70 \\
(3.03) \\
\end{array}$ & $\begin{array}{l}-40.81 \\
(2.31)\end{array}$ & $\begin{array}{c}-17.69 \\
(1.82) \\
\end{array}$ & $\begin{array}{c}-26.45 \\
(2.78) \\
\end{array}$ & $\begin{array}{c}-20.06 \\
(2.25)\end{array}$ \\
\hline$\overline{\mathrm{B}_{2} \mathrm{O}_{3}}$ & $\begin{array}{l}11.42 \\
(3.98)\end{array}$ & $\begin{array}{c}2.81 \\
(0.30) \\
\end{array}$ & $\begin{array}{l}12.82 \\
(1.23)\end{array}$ & $\begin{array}{c}1.95 \\
(0.20) \\
\end{array}$ & $\begin{array}{c}8.80 \\
(2.94) \\
\end{array}$ & $\begin{array}{c}1.01 \\
(0.24)\end{array}$ \\
\hline $\mathrm{CaO}$ & $\begin{array}{c}34.90 \\
(36.88)\end{array}$ & $\begin{array}{c}4.60 \\
(0.51)\end{array}$ & $\begin{array}{l}-7.79 \\
(1.79)\end{array}$ & $\begin{array}{c}3.33 \\
(0.33)\end{array}$ & $\begin{array}{c}-8.13 \\
(2.94)\end{array}$ & $\begin{array}{c}2.49 \\
(0.39) \\
\end{array}$ \\
\hline$\overline{\mathrm{Fe}_{2} \mathrm{O}_{3}}$ & (b) & $\begin{array}{c}-10.44 \\
(1.45)\end{array}$ & $\begin{array}{l}-8.39 \\
(3.98) \\
\end{array}$ & $\begin{array}{l}-8.25 \\
(0.87) \\
\end{array}$ & $\begin{array}{c}-26.08 \\
(7.80) \\
\end{array}$ & $\begin{array}{c}-9.93 \\
(1.09) \\
\end{array}$ \\
\hline FeO & (a) & (a) & (a) & (a) & $\begin{array}{c}38.60 \\
(23.44) \\
\end{array}$ & $\begin{array}{c}5.79 \\
(0.75) \\
\end{array}$ \\
\hline$\overline{\mathrm{Li}_{2} \mathrm{O}}$ & $\begin{array}{l}22.35 \\
(4.57) \\
\end{array}$ & $\begin{array}{l}10.02 \\
(1.20)\end{array}$ & $\begin{array}{l}10.63 \\
(1.28)\end{array}$ & $\begin{array}{c}7.50 \\
(0.75) \\
\end{array}$ & (a) & (a) \\
\hline $\mathrm{MgO}$ & $\begin{array}{c}27.65 \\
(21.08) \\
\end{array}$ & $\begin{array}{c}0.77 \\
(0.14) \\
\end{array}$ & $\begin{array}{c}7.01 \\
(1.46)\end{array}$ & $\begin{array}{c}0.38 \\
(0.10)\end{array}$ & (a) & (a) \\
\hline $\mathrm{Na}_{2} \mathrm{O}$ & $\begin{array}{c}22.45 \\
(3.66) \\
\end{array}$ & $\begin{array}{c}25.42 \\
(3.18)\end{array}$ & $\begin{array}{l}17.53 \\
(1.18)\end{array}$ & $\begin{array}{l}19.36 \\
(1.95) \\
\end{array}$ & $\begin{array}{l}17.31 \\
(2.78) \\
\end{array}$ & $\begin{array}{l}19.70 \\
(2.34)\end{array}$ \\
\hline$\overline{\mathrm{SiO}_{2}}$ & $\begin{array}{l}-2.98 \\
(1.01) \\
\end{array}$ & $\begin{array}{c}-4.87 \\
-(0.74) \\
\end{array}$ & $\begin{array}{l}-4.05 \\
(0.55) \\
\end{array}$ & $\begin{array}{l}-3.96 \\
(0.43) \\
\end{array}$ & $\begin{array}{l}-3.57 \\
(1.36) \\
\end{array}$ & $\begin{array}{l}-5.33 \\
(0.57) \\
\end{array}$ \\
\hline $\mathrm{ZrO}_{2}$ & (b) & $\begin{array}{l}-12.00 \\
(1.65) \\
\end{array}$ & $\begin{array}{l}-20.90 \\
(3.54)\end{array}$ & $\begin{array}{l}-9.45 \\
(0.99)\end{array}$ & (a) & (a) \\
\hline $\begin{array}{l}\text { Others } \\
\text { prevalent } \\
\text { values }\end{array}$ & $\begin{array}{l}-41.04^{(c)} \\
(11.54)\end{array}$ & $\begin{array}{l}0.64 \text { to } 0.76^{(\text {()) }} \\
(0.14 \text { to } 0.14) \\
0.76 \\
(0.14)\end{array}$ & $\begin{array}{c}0.98^{(c)} \\
(3.39)\end{array}$ & $\begin{array}{l}-2.57 \text { to } 18.75^{(\infty)} \\
(0.10 \text { to } 1.89) \\
0.37 \\
(0.10)\end{array}$ & (c) & (e) \\
\hline $\mathbf{R}^{2}$ & 0.899 & 0.741 & 0.810 & 0.400 & 0.880 & 0.730 \\
\hline $\mathrm{R}^{2}$ (ADJ) & 0.852 & 0.729 & 0.798 & 0.396 & 0.848 & 0.720 \\
\hline $\mathrm{R}^{2}$ (PRESS) & $0.709^{(n)}$ & 0.690 & 0.776 & 0.384 & 0.769 & 0.673 \\
\hline $\mathbf{s}$ & 0.489 & 0.662 & 0.677 & 1.169 & 0.638 & 0.867 \\
\hline
\end{tabular}

(a) Component not included or determined for glasses in this data set.

(b) $\mathrm{Fe}_{2} \mathrm{O}_{3}$ and $\mathrm{ZrO}_{2}$ were included in the Others component for fitting the first-order mixture model, since CVS-II Phase 4 consisted of experiments varying $\mathrm{SiO}_{2}, \mathrm{~B}_{2} \mathrm{O}_{3}, \mathrm{Na}_{2} \mathrm{O}, \mathrm{Li}_{2} \mathrm{O}, \mathrm{CaO}, \mathrm{MgO}$, and $\mathrm{Al}_{2} \mathrm{O}_{3}$ one-at-a-time. Hence, the effects of $\mathrm{Fe}_{2} \mathrm{O}_{3}$ and $\mathrm{ZrO}_{2}$ are highly correlated with the effect of Others, and cannot be separately estimated.

(c) The Others coefficient in the first-order mixture model may be considered to be an average coefficient for all the components contained in Others.

(d) Others coefficients for FEH models were computed by forming a weighted average of the coefficients for the individual Others components, where the relative proportions of the Others components were used as weights. This can yield different weighted averages across the data points in the data set, so the ranges of coefficient values and standard deviations are reported. For the CVS-II.4 and CVS(ail) data sets, the majority of glasses have the same relative proportions in Others as in CVS-I and other CVS-II phases, and these "prevalent" coefficients and standard deviations are also shown.

(c) No other components in this data set.

(f) Computed in a modified way, since the data set does not support fitting a $\mathrm{CaO}$ model term when glass CVS2-116 is deleted from the data set. The PRESS contribution with CVS2-116 deleted was obtained by including $\mathrm{CaO}$ in Others. $\mathrm{CaO}$ was retained in the model for the remaining PRESS contributions. 
Table 4. Coefficients, Coefficient Standard Deviations (in parentheses), and Goodness-of-Fit Statistics for First-Order Mixture Models and Rewritten Free Energy of Hydration Models for $\ln \left(\right.$ PCT Normalized B Release, $\mathrm{g} / \mathrm{m}^{2}$ ) (continued)

\begin{tabular}{|c|c|c|c|c|c|c|}
\hline \multirow[b]{2}{*}{ Component } & \multicolumn{2}{|c|}{ WV87 } & \multicolumn{2}{|c|}{ WV88 } & \multicolumn{2}{|c|}{ WVDP } \\
\hline & Mixture(a) & FEH & Mixture(a) & $\mathrm{FEH}$ & Mixture & FEH \\
\hline $\mathrm{Al}_{2} \mathrm{O}_{3}$ & $\begin{array}{l}-9.77 \\
(2.38)\end{array}$ & $\begin{array}{l}-6.86 \\
(2.06)\end{array}$ & $\begin{array}{r}-14.69 \\
(1.21) \\
\end{array}$ & $\begin{array}{l}-7.79 \\
(1.72)\end{array}$ & $\begin{array}{r}-14.07 \\
(8.50) \\
\end{array}$ & $\begin{array}{r}-21.16 \\
(2.21) \\
\end{array}$ \\
\hline $\mathrm{B}_{2} \mathrm{O}_{3}$ & $\begin{array}{c}3.18 \\
(1.89)\end{array}$ & $\begin{array}{l}-0.78 \\
(0.28) \\
\end{array}$ & $\begin{array}{c}1.79 \\
(0.85) \\
\end{array}$ & $\begin{array}{l}-0.69 \\
(0.16)\end{array}$ & $\begin{array}{r}14.53 \\
(1.81) \\
\end{array}$ & $\begin{array}{r}0.97 \\
(0.16) \\
\end{array}$ \\
\hline $\mathrm{BaO}$ & $\begin{array}{l}-6.28 \\
(1.99) \\
\end{array}$ & $\begin{array}{c}0.82 \\
(0.90) \\
\end{array}$ & $\begin{array}{c}3.51 \\
(1.20) \\
\end{array}$ & $\begin{array}{c}1.19 \\
(0.65) \\
\end{array}$ & (b) & (b) \\
\hline $\mathrm{CaO}$ & $\begin{array}{l}-6.28 \\
(1.99) \\
\end{array}$ & $\begin{array}{l}-0.36 \\
(0.45) \\
\end{array}$ & $\begin{array}{c}3.51 \\
(1.20) \\
\end{array}$ & $\begin{array}{l}-0.19 \\
(0.29) \\
\end{array}$ & (b) & (b) \\
\hline $\mathrm{Fe}_{2} \mathrm{O}_{3}$ & $\begin{array}{l}-0.29 \\
(1.90)\end{array}$ & $\begin{array}{l}-3.94 \\
(0.93) \\
\end{array}$ & $\begin{array}{l}-0.39 \\
(1.42) \\
\end{array}$ & $\begin{array}{l}-4.38 \\
(0.82) \\
\end{array}$ & $\begin{array}{r}-10.63 \\
(4.36) \\
\end{array}$ & $\begin{array}{r}-10.52 \\
(1.09) \\
\end{array}$ \\
\hline $\mathrm{K}_{2} \mathrm{O}$ & $\begin{array}{c}5.86 \\
(1.37) \\
\end{array}$ & $\begin{array}{c}7.54 \\
(3.48)\end{array}$ & $\begin{array}{c}5.26 \\
(0.60) \\
\end{array}$ & $\begin{array}{c}9.05 \\
(2.73)\end{array}$ & $\begin{array}{l}14.44 \\
(6.22) \\
\end{array}$ & $\begin{array}{l}31.32 \\
(3.33) \\
\end{array}$ \\
\hline $\mathrm{Li}_{2} \mathrm{O}$ & $\begin{array}{c}5.86 \\
(1.37) \\
\end{array}$ & $\begin{array}{c}0.93 \\
(0.94) \\
\end{array}$ & $\begin{array}{c}5.26 \\
(0.60) \\
\end{array}$ & $\begin{array}{r}1.32 \\
(0.69) \\
\end{array}$ & $\begin{array}{c}9.08 \\
(2.80) \\
\end{array}$ & $\begin{array}{c}7.24 \\
(0.79) \\
\end{array}$ \\
\hline $\mathrm{MgO}$ & $\begin{array}{l}-6.28 \\
(1.99) \\
\end{array}$ & $\begin{array}{l}-1.27 \\
(0.10)\end{array}$ & $\begin{array}{c}3.51 \\
(1.20) \\
\end{array}$ & $\begin{array}{l}-1.26 \\
(0.05) \\
\end{array}$ & (b) & (b) \\
\hline $\mathrm{MnO}$ & $\begin{array}{c}1.10 \\
(3.09)\end{array}$ & $\begin{array}{c}0.98 \\
(0.96)\end{array}$ & $\begin{array}{c}8.98 \\
(2.47)\end{array}$ & $\begin{array}{c}1.38 \\
(0.70) \\
\end{array}$ & (b) & (b) \\
\hline $\mathrm{Na}_{2} \mathrm{O}$ & $\begin{array}{c}5.86 \\
(1.37) \\
\end{array}$ & $\begin{array}{c}4.60 \\
(2.35) \\
\end{array}$ & $\begin{array}{c}5.26 \\
(0.60) \\
\end{array}$ & $\begin{array}{c}5.60 \\
(1.82) \\
\end{array}$ & $\begin{array}{l}7.29 \\
(3.50) \\
\end{array}$ & $\begin{array}{l}20.60 \\
(2.20) \\
\end{array}$ \\
\hline$\overline{\mathrm{P}_{2} \mathrm{O}_{5}}$ & $\begin{array}{l}-0.77 \\
(4.95)\end{array}$ & $\begin{array}{c}1.25 \\
(1.06)\end{array}$ & $\begin{array}{l}-9.96 \\
(2.17)\end{array}$ & $\begin{array}{c}1.69 \\
(0.78) \\
\end{array}$ & (b) & (b) \\
\hline $\mathrm{SiO}_{2}$ & $\begin{array}{l}-4.49 \\
(0.60) \\
\end{array}$ & $\begin{array}{l}-2.61 \\
(0.42) \\
\end{array}$ & $\begin{array}{l}-3.65 \\
(0.38) \\
\end{array}$ & $\begin{array}{l}-2.83 \\
(0.41) \\
\end{array}$ & $\begin{array}{l}-6.09 \\
(0.98) \\
\end{array}$ & $\begin{array}{l}-5.69 \\
(0.58) \\
\end{array}$ \\
\hline $\mathrm{ThO}_{2}$ & $\begin{array}{l}13.09 \\
(6.38) \\
\end{array}$ & $\begin{array}{l}-4.53 \\
(1.16) \\
\end{array}$ & $\begin{array}{c}8.13 \\
(4.90) \\
\end{array}$ & $\begin{array}{l}-5.07 \\
(1.00)\end{array}$ & $\begin{array}{c}-5.36 \\
(18.56)\end{array}$ & $\begin{array}{r}-12.67 \\
(1.31) \\
\end{array}$ \\
\hline $\mathrm{U}_{3} \mathrm{O}_{8}$ & $\begin{array}{c}15.79 \\
(66.78) \\
\end{array}$ & $\begin{array}{r}0.90 \\
(0.93) \\
\end{array}$ & $\begin{array}{c}50.44 \\
(21.10)\end{array}$ & $\begin{array}{r}1.28 \\
(0.68) \\
\end{array}$ & (b) & (b) \\
\hline $\mathrm{ZrO}_{2}$ & (b) & (b) & (b) & (b) & $\begin{array}{c}-31.14 \\
(30.22) \\
\end{array}$ & $\begin{array}{r}-11.87 \\
(1.23) \\
\end{array}$ \\
\hline Others & $\begin{array}{l}3.52^{(c)} \\
(2.07)\end{array}$ & $\begin{array}{l}-2.64 \text { to }-2.48^{(0)} \\
(0.37 \text { to } 0.44)\end{array}$ & $\begin{array}{l}-3.05^{(c)} \\
(0.92)\end{array}$ & $\begin{array}{l}-2.81 \text { to }-2.78^{(\Phi)} \\
(0.40 \text { to } 0.41)\end{array}$ & (c) & $\begin{array}{l}0.01 \text { to } 1.62^{(\Phi)} \\
(0.10 \text { to } 0.22)\end{array}$ \\
\hline $\mathrm{R}^{2}$ & 0.917 & 0.326 & 0.980 & 0.506 & 0.842 & 0.620 \\
\hline $\mathrm{R}^{2}$ (ADJ) & 0.750 & 0.278 & 0.939 & 0.471 & 0.816 & 0.614 \\
\hline $\mathrm{R}^{2}$ (PRESS) & 0.165 & 0.098 & 0.793 & 0.361 & 0.780 & 0.594 \\
\hline s & 0.091 & 0.155 & 0.066 & 0.195 & 0.493 & 0.714 \\
\hline
\end{tabular}

(a) $\mathrm{BaO}+\mathrm{CaO}+\mathrm{MgO}$ and $\mathrm{K}_{2} \mathrm{O}+\mathrm{Li}_{2} \mathrm{O}+\mathrm{Na}_{2} \mathrm{O}$ were each treated as combined components, so that the same coefficients are listed for the separate components in each group.

(b) Included in the Others component for this data set-see Table 5.

(c) The Others coefficient in the first-order mixture model may be considered to be an average coefficient for all the components contained in Others.

(d) Others coefficients for FEH models were computed by forming a weighted average of the coefficients for the individual Others components, where the relative proportions of the Others components were used as weights. This can yield different weighted averages across the data points in the data set, so the ranges of coefficient values and standard deviations are reported.

(e) All Others components have constant mass fraction values except for minor variations in three glasses, and there is little variation in mole fractions. This is insufficient to estimate a separate coefficient for Others. The nine components varied in the WVDP data were renormalized to a total mole fraction of 1.0 and then the first-order mixture model was fitted. 
Table 4. Coefficients, Coefficient Standard Deviations (in parentheses), and Goodness-of-Fit Statistics for First-Order Mixture Models and Rewritten Free Energy of Hydration Models for $\ln$ (PCT Normalized B Release, $\mathrm{g} / \mathrm{m}^{2}$ ) (continued)

\begin{tabular}{|c|c|c|c|c|c|}
\hline \multirow[b]{2}{*}{ Component } & \multicolumn{2}{|c|}{ SRTC 137 } & \multicolumn{2}{|c|}{ SRTC 118} & SRTC 111 \\
\hline & Mixture & FEH & Mixture & FEH & Mixture. $\mathrm{FEH}^{(d)}$ \\
\hline $\mathrm{Al}_{2} \mathrm{O}_{3}$ & $\begin{array}{l}-32.03 \\
(2.04) \\
\end{array}$ & $\begin{array}{r}-27.99 \\
(1.31) \\
\end{array}$ & $\begin{array}{c}-30.48 \\
(2.77) \\
\end{array}$ & $\begin{array}{r}-30.28 \\
(1.53) \\
\end{array}$ & $\begin{array}{r}-22.29 \\
(3.26) \\
\end{array}$ \\
\hline $\mathrm{B}_{2} \mathrm{O}_{3}$ & $\begin{array}{c}4.17 \\
(1.83) \\
\end{array}$ & $\begin{array}{c}1.54 \\
(0.12)\end{array}$ & $\begin{array}{c}6.14 \\
(2.52)\end{array}$ & $\begin{array}{c}1.78 \\
(0.13) \\
\end{array}$ & $\begin{array}{c}4.44 \\
(2.54) \\
\end{array}$ \\
\hline $\mathrm{CaO}$ & $\begin{array}{l}-2.08 \\
(3.42) \\
\end{array}$ & $\begin{array}{c}3.62 \\
(0.21)\end{array}$ & $\begin{array}{c}20.29 \\
(11.21) \\
\end{array}$ & $\begin{array}{r}4.04 \\
(0.25) \\
\end{array}$ & $\begin{array}{c}5.40 \\
(11.16)\end{array}$ \\
\hline $\mathrm{Cr}_{2} \mathrm{O}_{3}$ & $\begin{array}{l}109.45 \\
(65.13)\end{array}$ & $\begin{array}{r}-12.20 \\
(0.55)\end{array}$ & $\begin{array}{c}76.65 \\
(71.02) \\
\end{array}$ & $\begin{array}{c}-13.13 \\
(0.65) \\
\end{array}$ & $\begin{array}{c}13.35 \\
(70.85) \\
\end{array}$ \\
\hline $\mathrm{CuO}$ & $\begin{array}{l}133.56 \\
(38.83) \\
\end{array}$ & $\begin{array}{c}1.69 \\
(0.12)\end{array}$ & $\begin{array}{c}72.90 \\
(44.36) \\
\end{array}$ & $\begin{array}{r}1.95 \\
(0.14) \\
\end{array}$ & $\begin{array}{c}44.86 \\
(48.43) \\
\end{array}$ \\
\hline $\mathrm{Fe}_{2} \mathrm{O}_{3}$ & $\begin{array}{r}-19.44 \\
(3.06) \\
\end{array}$ & $\begin{array}{r}-13.80 \\
(0.63)\end{array}$ & $\begin{array}{r}-22.97 \\
(4.61)\end{array}$ & $\begin{array}{r}-14.87 \\
(0.74) \\
\end{array}$ & $\begin{array}{r}-14.49 \\
(4.64) \\
\end{array}$ \\
\hline $\mathrm{FeO}$ & $\begin{array}{l}29.35 \\
(4.72) \\
\end{array}$ & $\begin{array}{c}8.23 \\
(0.43) \\
\end{array}$ & $\begin{array}{l}24.73 \\
(5.25) \\
\end{array}$ & $\begin{array}{c}9.05 \\
(0.50) \\
\end{array}$ & $\begin{array}{c}61.42 \\
(12.32) \\
\end{array}$ \\
\hline $\mathrm{K}_{2} \mathrm{O}$ & $\begin{array}{r}-30.08 \\
(7.37) \\
\end{array}$ & $\begin{array}{l}42.04 \\
(2.05)\end{array}$ & $\begin{array}{r}-21.89 \\
(8.19)\end{array}$ & $\begin{array}{l}45.76 \\
(2.39)\end{array}$ & $\begin{array}{c}-14.01 \\
(8.03)\end{array}$ \\
\hline $\mathrm{Li}_{2} \mathrm{O}$ & $\begin{array}{r}5.39 \\
(2.08) \\
\end{array}$ & $\begin{array}{c}9.90 \\
(0.51) \\
\end{array}$ & $\begin{array}{c}5.41 \\
(3.04) \\
\end{array}$ & $\begin{array}{l}10.85 \\
(0.59)\end{array}$ & $\begin{array}{c}3.20 \\
(2.98) \\
\end{array}$ \\
\hline $\mathrm{MgO}$ & $\begin{array}{c}4.85 \\
(5.06)\end{array}$ & $\begin{array}{l}-0.82 \\
(0.05)\end{array}$ & $\begin{array}{c}7.91 \\
(6.19)\end{array}$ & $\begin{array}{l}-0.78 \\
(0.05)\end{array}$ & $\begin{array}{l}19.19 \\
(8.93)\end{array}$ \\
\hline $\mathrm{MnO}$ & $\begin{array}{l}18.75 \\
(7.10)\end{array}$ & $\begin{array}{l}10.14 \\
(0.52) \\
\end{array}$ & $\begin{array}{l}14.12 \\
(7.43)\end{array}$ & $\begin{array}{l}11.12 \\
(0.61)\end{array}$ & $\begin{array}{l}-3.43 \\
(9.37)\end{array}$ \\
\hline $\mathrm{Na}_{2} \mathrm{O}$ & $\begin{array}{l}21.07 \\
(1.50)\end{array}$ & $\begin{array}{l}27.73 \\
(1.36)\end{array}$ & $\begin{array}{l}19.56 \\
(1.82)\end{array}$ & $\begin{array}{l}30.22 \\
(1.59)\end{array}$ & $\begin{array}{l}16.98 \\
(1.87)\end{array}$ \\
\hline $\mathrm{NiO}$ & $\begin{array}{l}-48.18 \\
(14.04)\end{array}$ & $\begin{array}{l}-5.09 \\
(0.22) \\
\end{array}$ & $\begin{array}{l}-40.15 \\
(15.86) \\
\end{array}$ & $\begin{array}{l}-5.41 \\
(0.25) \\
\end{array}$ & $\begin{array}{c}-0.09 \\
(21.19) \\
\end{array}$ \\
\hline $\mathrm{SiO}_{2}$ & $\begin{array}{l}-3.62 \\
(0.38) \\
\end{array}$ & $\begin{array}{l}-7.35 \\
(0.32) \\
\end{array}$ & $\begin{array}{l}-4.20 \\
(0.68)\end{array}$ & $\begin{array}{l}-7.87 \\
(0.38) \\
\end{array}$ & $\begin{array}{l}-4.47 \\
(0.65) \\
\end{array}$ \\
\hline $\mathrm{TiO}_{2}$ & $\begin{array}{c}61.91 \\
(12.68) \\
\end{array}$ & $\begin{array}{l}-14.85 \\
(0.68) \\
\end{array}$ & $\begin{array}{c}56.79 \\
(12.78) \\
\end{array}$ & $\begin{array}{l}-16.01 \\
(0.80) \\
\end{array}$ & $\begin{array}{c}41.81 \\
(12.74) \\
\end{array}$ \\
\hline Others & $\begin{array}{c}-54.24^{(())} \\
(7.22)\end{array}$ & $\begin{array}{c}-15.60 \text { to } 18.94^{(\mathrm{b})} \\
(0.05 \text { to } 0.94)\end{array}$ & $\begin{array}{l}10.92^{(2)} \\
(20.75)\end{array}$ & $\begin{array}{c}-16.82 \text { to } 20.68^{(0)} \\
(0.05 \text { to } 1.10)\end{array}$ & $\begin{array}{c}30.01 \\
(20.95)\end{array}$ \\
\hline $\mathrm{R}^{2}$ & $0.961^{(c)}$ & 0.763 & $0.961^{(c)}$ & 0.764 & 0.968 \\
\hline $\mathrm{R}^{2}(\mathrm{ADJ})$ & 0.956 & 0.762 & 0.955 & 0.762 & 0.963 \\
\hline $\mathrm{R}^{2}$ (PRESS) & 0.935 & 0.751 & 0.937 & 0.752 & 0.949 \\
\hline s & 0.251 & 0.583 & 0.237 & 0.547 & 0.215 \\
\hline
\end{tabular}

(a) The Others coefficient in the first-order mixture model may be considered to be an average coefficient for all the components contained in Others.

(b) Others coefficients for FEH models were computed by forming a weighted average of the coefficients for the individual Others components, where the relative proportions of the Others components were used as weights. This can yield different weighted averages across the data points in the data set, so the ranges of coefficient values and standard deviations are reported.

(c) Although the various $\mathrm{R}^{2}$ values are nearly identical for the 137-, 118-, and 111-observation data sets, the models for 118 and 111 observations fit those data sets considerably better than the 137-observation model.

(d) The FEH model for SRTC 111 is essentially the same as for SRTC 118, and so is not reported here. 
Table 5. Coefficients of Others Components for Free Energy of Hydration Models Written in the Form of First-Order Mixture Models for $\ln \left(\right.$ PCT Normalized B Release in $\mathrm{g} / \mathrm{m}^{2}$ )

\begin{tabular}{|c|c|c|c|c|c|c|}
\hline $\begin{array}{l}\text { Others } \\
\text { Component } t^{(a)}\end{array}$ & CVS-I & CVS-II.I & CVS-II.2 & CVS-II.3 & CVS-II.4 & CVS(all) \\
\hline $\mathrm{Ag}_{2} \mathrm{O}$ & (b) & (b) & (b) & -2.73 & (b) & -2.31 \\
\hline $\mathrm{As}_{2} \mathrm{O}_{3}$ & (b) & (b) & (b) & -17.52 & (b) & -16.04 \\
\hline $\mathrm{BaO}$ & 9.34 & 4.79 & 3.60 & 7.46 & 9.57 & 7.15 \\
\hline $\mathrm{CdO}$ & -1.49 & -1.68 & -0.78 & -1.97 & -1.81 & -1.60 \\
\hline $\mathrm{Ce}_{2} \mathrm{O}_{3}$ & 20.34 & 11.36 & 8.06 & 17.04 & 21.12 & 16.05 \\
\hline $\mathrm{CoO}$ & (b) & (b) & (b) & 0.07 & (b) & 0.29 \\
\hline $\mathrm{Cr}_{2} \mathrm{O}_{3}$ & -8.39 & -5.80 & -3.58 & -7.98 & -9.06 & -7.19 \\
\hline $\mathrm{Cs}_{2} \mathrm{O}$ & 38.21 & 22.03 & 15.31 & 32.60 & 39.89 & 30.49 \\
\hline $\mathrm{CuO}$ & 3.03 & 1.02 & 1.05 & 1.96 & 2.94 & 2.05 \\
\hline$F$ & -2.36 & -2.20 & -1.14 & -2.73 & -2.72 & -2.31 \\
\hline $\mathrm{FeO}$ & (b) & (b) & (b) & (b) & (b) & (b) \\
\hline $\mathrm{K}_{2} \mathrm{O}$ & (b) & (b) & (b) & 30.85 & (b) & 28.87 \\
\hline $\mathrm{La}_{2} \mathrm{O}_{3}$ & 21.76 & 12.21 & 8.64 & 18.27 & 22.61 & 17.19 \\
\hline $\mathrm{MnO}$ & 9.98 & 5.17 & 3.86 & 8.01 & 10.23 & 7.66 \\
\hline $\mathrm{MoO}_{3}$ & -10.67 & -7.16 & -4.51 & -9.96 & -11.45 & -9.03 \\
\hline $\mathrm{Nd}_{2} \mathrm{O}_{3}$ & 16.71 & 9.19 & 6.59 & 13.88 & 17.31 & 13.11 \\
\hline $\mathrm{NiO}$ & -2.55 & -2.31 & -1.21 & -2.89 & -2.92 & -2.46 \\
\hline $\mathrm{P}_{2} \mathrm{O}_{5}$ & 11.04 & 5.80 & 4.29 & 8.94 & 11.35 & 8.53 \\
\hline $\mathrm{PbO}$ & (b) & (b) & (b) & -11.97 & (b) & -10.89 \\
\hline PdO & -2.36 & -2.20 & -1.14 & -2.73 & -2.72 & -2.31 \\
\hline $\mathrm{PdO}_{2}$ & (b) & (b) & (b) & (b) & (b) & (b) \\
\hline $\mathrm{Pr}_{6} \mathrm{O}_{11}$ & -2.36 & -2.20 & $-1,14$ & -2.73 & -2.72 & -2.31 \\
\hline $\mathrm{Rb}_{2} \mathrm{O}$ & 41.16 & 23.80 & 16.50 & 35.17 & 43.00 & 32.88 \\
\hline $\mathrm{RhO}_{2}$ & (b) & (b) & (b) & (b) & (b) & (b) \\
\hline $\mathrm{Rh}_{2} \mathrm{O}_{3}$ & -2.36 & -2.20 & -1.14 & -2.73 & -2.72 & -2.31 \\
\hline $\mathrm{RuO}_{2}$ & -31.28 & -19.48 & -12.86 & -27.91 & -33.10 & -25.69 \\
\hline $\mathrm{SO}_{3}$ & -2.36 & -2.20 & -1.14 & -2.73 & -2.72 & -2.31 \\
\hline $\mathrm{Sb}_{2} \mathrm{O}_{3}$ & (b) & (b) & (b) & 13.78 & (b) & 13.02 \\
\hline $\mathrm{SeO}_{2}$ & (b) & (b) & (b) & 0.70 & (b) & 0.87 \\
\hline $\mathrm{Sm}_{2} \mathrm{O}_{3}$ & -2.36 & -2.20 & -1.14 & -2.73 & -2.72 & -2.31 \\
\hline SrO & 7.59 & 3.75 & 2.90 & 5.94 & 7.73 & 5.74 \\
\hline $\mathrm{TeO}_{2}$ & (b) & (b) & (b) & -8.01 & (b) & -7.21 \\
\hline $\mathrm{ThO}_{2}$ & (b) & (b) & (b) & (b) & (b) & (b) \\
\hline $\mathrm{TiO}_{2}$ & (b) & (b) & (b) & -9.88 & (b) & -8.95 \\
\hline $\mathrm{U}_{3} \mathrm{O}_{8}$ & (b) & (b) & 3.73 & (b) & (b) & 7.39 \\
\hline $\mathrm{WO}_{3}$ & (b) & (b) & (b) & (b) & (b) & (b) \\
\hline $\mathrm{Y}_{2} \mathrm{O}_{3}$ & 4.16 & 1.69 & 1.50 & 2.94 & 4.12 & 2.96 \\
\hline $\mathrm{ZnO}$ & (b) & -2.48 & -1.33 & -3.13 & -3.21 & -2.68 \\
\hline
\end{tabular}

(a) Component coefficients were computed according to the two parts of Equation (5d), depending on whether or not a component is in $S$, the group of components assumed to be associated with $\mathrm{SiO}_{2}$ in glass dissolution reactions. Authorization has not been given to publish which components are in the group $S$. If and when such authorization is received, a revised version of this table may be requested from the first author.

(b) Component was not included or determined for glasses in this data set. 
Table 5. Coefficients of Others Components for Free Energy of Hydration Models Written in the Form of First-Order Mixture Models for $\ln \left(\mathrm{PCT}\right.$ Normalized B Release in $\mathrm{g} / \mathrm{m}^{2}$ ) (continued)

\begin{tabular}{|c|c|c|c|c|c|c|}
\hline $\begin{array}{l}\text { Others } \\
\text { Component }{ }^{(1)}\end{array}$ & Ramsey & WV87 & WV88 & WVDP & $\begin{array}{l}\text { SRTC Homo. } \\
\text { All } 137 \text { Obs. }\end{array}$ & $\begin{array}{l}\text { SRTC Homo. } \\
118 \text { Obs. }\end{array}$ \\
\hline $\mathrm{Ag}_{2} \mathrm{O}$ & (b) & (b) & (b) & (b) & (b) & (b) \\
\hline $\mathrm{As}_{2} \mathrm{O}_{3}$ & (b) & (b) & (b) & (b) & (b) & (b) \\
\hline $\mathrm{BaO}$ & (b) & (c) & (c) & 6.84 & 9.37 & 10.28 \\
\hline $\mathrm{CaO}$ & (c) & (c) & (c) & 2.53 & (c) & (c) \\
\hline CdO & (b) & (b) & (b) & (b) & (b) & (b) \\
\hline $\mathrm{Ce}_{2} \mathrm{O}_{3}$ & (b) & 3.58 & 4.41 & 16.87 & 22.75 & 24.81 \\
\hline $\mathrm{CoO}$ & (b) & (b) & (b) & -0.89 & (b) & (b) \\
\hline $\mathrm{Cr}_{2} \mathrm{O}_{3}$ & (b) & -3.61 & -4.00 & -9.32 & (c) & (c) \\
\hline $\mathrm{Cs}_{2} \mathrm{O}$ & (b) & 8.04 & 9.63 & 33.15 & 44.48 & 48.41 \\
\hline $\mathrm{CuO}$ & (b) & -0.75 & -0.66 & 1.09 & (c) & (c) \\
\hline F & (b) & (b) & (b) & (b) & (b) & (b) \\
\hline $\mathrm{FeO}$ & (c) & (b) & (b) & (b) & (c) & (c) \\
\hline $\mathrm{Fe}_{2} \mathrm{O}_{3}$ & (c) & (c) & (c) & (c) & (c) & (c) \\
\hline $\mathrm{K}_{2} \mathrm{O}$ & (b) & (c) & (c) & (c) & (c) & (c) \\
\hline $\mathrm{La}_{2} \mathrm{O}_{3}$ & (b) & 3.93 & 4.82 & 18.16 & 24.47 & 26.68 \\
\hline $\mathrm{MgO}^{\circ}$ & (b) & (c) & (c) & -0.80 & (c) & (c) \\
\hline $\mathrm{MnO}$ & (b) & (c) & (c) & 7.42 & (c) & (c) \\
\hline $\mathrm{MoO}_{3}$ & (b) & -4.18 & -4.66 & -11.39 & -14.96 & -16.14 \\
\hline $\mathrm{Nd}_{2} \mathrm{O}_{3}$ & (b) & 2.67 & 3.35 & 13.56 & 18.33 & 20.02 \\
\hline $\mathrm{NiŌ}$ & (b) & -2.15 & -2.29 & -3.99 & (c) & (c) \\
\hline $\mathrm{P}_{2} \mathrm{O}_{5}$ & (b) & (c) & (c) & 8.39 & 11.44 & 12.53 \\
\hline Pbo & (b) & (b) & (b) & (b) & -17.77 & -19.18 \\
\hline PdO & (b) & (b) & (b) & -3.82 & (b) & (b) \\
\hline $\mathrm{PdO}_{2}$ & (b) & (b) & (b) & (b) & (b) & (b) \\
\hline $\mathrm{Pr}_{6} \mathrm{O}_{11}$ & (b) & -2.10 & -2.23 & -3.82 & (b) & (b) \\
\hline $\mathrm{Rb}_{2} \mathrm{O}$ & (b) & (b) & (b) & (b) & (b) & (b) \\
\hline $\mathrm{RhO}_{2}$ & (b) & (b) & (b) & -3.82 & (b) & (b) \\
\hline $\mathrm{Rh}_{2} \mathrm{O}_{3}$ & (b) & (b) & (b) & (b) & (b) & (b) \\
\hline $\mathrm{RuO}_{2}$ & (b) & (b) & (b) & -30.18 & (b) & (b) \\
\hline $\mathrm{SO}_{3}$ & (b) & -2.10 & -2.23 & -3.82 & (b) & (b) \\
\hline $\mathrm{Sb}_{2} \mathrm{O}_{3}$ & (b) & (b) & (b) & (b) & (b) & (b) \\
\hline $\mathrm{SeO}_{2}$ & (b) & (b) & (b) & (b) & (b) & (b) \\
\hline $\mathrm{Sm}_{2} \mathrm{O}_{3}$ & (b) & -2.10 & -2.23 & -3.82 & (b) & (b) \\
\hline $\mathrm{SrO}$ & (b) & 0.39 & 0.68 & 5.25 & 7.24 & 7.98 \\
\hline $\mathrm{TeO}_{2}$ & (b) & (b) & (b) & (b) & (b) & (b) \\
\hline $\mathrm{ThO}_{2}$ & (b) & (c) & (c) & (c) & (b) & (b) \\
\hline $\mathrm{TiO}_{2}$ & (b) & -4.15 & -4.63 & -11.31 & (c) & (c) \\
\hline $\mathrm{U}_{3} \mathrm{O}_{8}$ & (b) & (c) & (c) & 7.11 & (b) & (b) \\
\hline $\mathrm{WO}_{3}$ & (b) & (b) & (b) & (b) & (b) & (b) \\
\hline $\mathrm{Y}_{2} \mathrm{O}_{3}$ & (b) & -0.47 & -0.33 & 2.12 & (b) & (b) \\
\hline $\mathrm{ZnO}$ & (b) & -2.22 & -2.37 & -4.25 & -5.43 & -5.78 \\
\hline $\mathrm{ZrO}_{2}$ & (b) & -4.31 & -4.81 & (c) & -15.60 & -16.82 \\
\hline
\end{tabular}

(a) Component coefficients were computed according to the two parts of Equation (5d), depending on whether or not a component is in $S$, the group of components assumed to be associated with $\mathrm{SiO}_{2}$ in glass dissolution reactions. Authorization has not been given to publish which components are in the group $S$. If and when such authorization is received, a revised version of this table may be requested from the first author.

(b) Component was not included or determined for glasses in this data set.

(c) Component was not in Others component for this data set--see Table 4 for coefficient. 
Table 6. Coefficients, Coefficient Standard Deviations (in parentheses), and Goodness-of-Fit Statistics for Reduced Second-Order Mixture Models for $\ln$ (PCT Normalized B Release, $\mathrm{g} / \mathrm{m}^{2}$ )

\begin{tabular}{|c|c|c|c|c|c|}
\hline \multicolumn{2}{|c|}{ WVDP } & \multicolumn{2}{|c|}{ CVS(all) } & \multicolumn{2}{|c|}{ RAMSEY } \\
\hline Model Term & $\begin{array}{c}\text { Coefficient } \\
\text { (sd) }\end{array}$ & Model Term & $\begin{array}{l}\text { Coefficient } \\
\text { (sd) }\end{array}$ & Model Term & $\begin{array}{l}\text { Coefficient } \\
\text { (sd) }\end{array}$ \\
\hline $\mathrm{Al}_{2} \mathrm{O}_{3}$ & $\begin{array}{c}-21.89 \\
(6.62)\end{array}$ & $\mathrm{Al}_{2} \mathrm{O}_{3}$ & $\begin{array}{c}-94.71 \\
(7.85) \\
\end{array}$ & $\mathrm{Al}_{2} \mathrm{O}_{3}$ & $\begin{array}{c}-56.16 \\
(8.57) \\
\end{array}$ \\
\hline $\mathrm{B}_{2} \mathrm{O}_{3}$ & $\begin{array}{l}40.02 \\
(6.83)\end{array}$ & $\mathrm{B}_{2} \mathrm{O}_{3}$ & $\begin{array}{l}11.78 \\
(1.50)\end{array}$ & $\mathrm{B}_{2} \mathrm{O}_{3}$ & $\begin{array}{c}9.31 \\
(2.35) \\
\end{array}$ \\
\hline $\mathrm{CaO}$ & (b) & $\mathrm{CaO}$ & $\begin{array}{l}13.55 \\
(4.67) \\
\end{array}$ & $\mathrm{CaO}$ & $\begin{array}{l}-7.63 \\
(2.33) \\
\end{array}$ \\
\hline $\mathrm{Fe}_{2} \mathrm{O}_{3}$ & $\begin{array}{l}-73.47 \\
(27: 79) \\
\end{array}$ & $\mathrm{Fe}_{2} \mathrm{O}_{3}$ & $\begin{array}{c}-18.85 \\
(4.18)\end{array}$ & $\mathrm{Fe}_{2} \mathrm{O}_{3}$ & $\begin{array}{c}-25.59 \\
(6.35) \\
\end{array}$ \\
\hline $\mathrm{FeO}$ & (a) & $\mathrm{FeO}$ & (a) & $\mathrm{FeO}$ & $\begin{array}{c}10.28 \\
(20.78) \\
\end{array}$ \\
\hline $\mathrm{K}_{2} \mathrm{O}$ & $\begin{array}{l}16.04 \\
(5.22) \\
\end{array}$ & $\mathrm{K}_{2} \mathrm{O}$ & (b) & $\mathrm{K}_{2} \mathrm{O}$ & (a) \\
\hline $\mathrm{Li}_{2} \mathrm{O}$ & $\begin{array}{c}27.47 \\
(9.45)\end{array}$ & $\mathrm{Li}_{2} \mathrm{O}$ & $\begin{array}{l}11.30 \\
(1.03)\end{array}$ & $\mathrm{Li}_{2} \mathrm{O}$ & (a) \\
\hline $\mathrm{MgO}$ & (b) & $\mathrm{MgO}$ & $\begin{array}{l}11.54 \\
(1.63)\end{array}$ & $\mathrm{MgO}$ & (a) \\
\hline $\mathrm{Na}_{2} \mathrm{O}$ & $\begin{array}{c}7.87 \\
(3.07) \\
\end{array}$ & $\mathrm{Na}_{2} \mathrm{O}$ & $\begin{array}{l}20.48 \\
(1.13)\end{array}$ & $\mathrm{Na}_{2} \mathrm{O}$ & $\begin{array}{l}14.25 \\
(2.33)\end{array}$ \\
\hline $\mathrm{SiO}_{2}$ & $\begin{array}{l}-5.18 \\
(1.38) \\
\end{array}$ & $\mathrm{SiO}_{2}$ & $\begin{array}{c}-3.44 \\
(0.51) \\
\end{array}$ & $\mathrm{SiO}_{2}$ & $\begin{array}{l}-1.24 \\
(1.22) \\
\end{array}$ \\
\hline $\mathrm{ThO}_{2}$ & $\begin{array}{c}-240.17 \\
(72.48) \\
\end{array}$ & $\mathrm{ThO}_{2}$ & (a) & $\mathrm{ThO}_{2}$ & (a) \\
\hline $\mathrm{ZrO}_{2}$ & $\begin{array}{l}-41.61 \\
(26.97) \\
\end{array}$ & $\mathrm{ZrO}_{2}$ & $\begin{array}{c}-25.34 \\
(2.81)\end{array}$ & $\mathrm{ZrO}_{2}$ & (a) \\
\hline Others & (c) & Others & $\begin{array}{l}3.43^{(0)} \\
(2.65)\end{array}$ & Others & (e) \\
\hline $\mathrm{Fe}_{2} \mathrm{O}_{3} * \mathrm{Fe}_{2} \mathrm{O}_{3}$ & $\begin{array}{r}805.57 \\
(221.73) \\
\end{array}$ & $\mathrm{Al}_{2} \mathrm{O}_{3} * \mathrm{Al}_{2} \mathrm{O}_{3}$ & $\begin{array}{l}406.09 \\
(54.04) \\
\end{array}$ & $\mathrm{Al}_{2} \mathrm{O}_{3} * \mathrm{Al}_{2} \mathrm{O}_{3}$ & $\begin{array}{l}185.40 \\
(59.64) \\
\end{array}$ \\
\hline $\mathrm{B}_{2} \mathrm{O}_{3} * \mathrm{~K}_{2} \mathrm{O}$ & $\begin{array}{c}-228.91 \\
(82.04) \\
\end{array}$ & $\mathrm{Na}_{2} \mathrm{O} * \mathrm{CaO}$ & $\begin{array}{c}-111.56 \\
(27.93) \\
\end{array}$ & $\mathrm{Al}_{2} \mathrm{O}_{3}{ }^{*} \mathrm{Fe}_{2} \mathrm{O}_{3}$ & $\begin{array}{l}186.30 \\
(72.80) \\
\end{array}$ \\
\hline $\mathrm{ThO}_{2} * \mathrm{ThO}_{2}$ & $\begin{array}{l}11850.0 \\
(3870.25)\end{array}$ & $\mathrm{B}_{2} \mathrm{O}_{3} * \mathrm{CaO}$ & $\begin{array}{l}-82.09 \\
(26.72) \\
\end{array}$ & & \\
\hline $\mathrm{B}_{2} \mathrm{O}_{3} * \mathrm{Li}_{2} \mathrm{O}$ & $\begin{array}{r}-145.12 \\
(70.02) \\
\end{array}$ & $\mathrm{MgO}^{*} \mathrm{Al}_{2} \mathrm{O}_{3}$ & $\begin{array}{c}-109.80 \\
(37.00) \\
\end{array}$ & & \\
\hline & & $\mathrm{Fe}_{2} \mathrm{O}_{3} * \mathrm{Al}_{2} \mathrm{O}_{3}$ & $\begin{array}{c}459.62 \\
(137.24) \\
\end{array}$ & & \\
\hline & & $\mathrm{B}_{2} \mathrm{O}_{3}^{*} \mathrm{Al}_{2} \mathrm{O}_{3}$ & $\begin{array}{r}85.79 \\
(31.74) \\
\end{array}$ & & \\
\hline $\mathrm{R}^{2}$ & 0.923 & & 0.892 & & 0.931 \\
\hline $\mathrm{R}^{2}(\mathrm{ADJ})$ & 0.903 & & 0.879 & & 0.905 \\
\hline $\mathrm{R}^{2}$ (PRESS) & 0.874 & & 0.860 & & 0.825 \\
\hline s & 0.358 & & 0.523 & & 0.504 \\
\hline
\end{tabular}

(a) Component was not included or determined for glasses in this data set.

(b) Included in Others component for this data set.

(c) Essentially only nine components were varied in the data set, and the mole fractions of these nine components were renormalized to a total mole fraction of 1.0 prior to fitting the model.

(d) The Others coefficient in the first-order mixture model may be considered to be an average coefficient for all the components contained in Others.

(e) No other components are in this data set. 


\section{Discussion}

The following subsections discuss various aspects of the first-order mixture, second-order mixture, and free energy of hydration models fitted to the data sets described in Section 4.

\subsection{Free Energy of Hydration Model Results}

Table 3 shows that the fitted FEH models have $R^{2}$ values between 0.113 and 0.764 [i.e., the models account for between $11.3 \%$ and $76.4 \%$ of the variation in $\ln (\mathrm{NR})$ values in the various data sets]. The $\mathrm{R}^{2}$ (ADJ) value for the model fitted to each data set is close to the corresponding $R^{2}$ value since, with only two coefficients estimated from each data set, it is unlikely the data are overfitted (i.e., that there are unneeded coefficients). $R^{2}$ (PRESS) values are reasonably close to the corresponding $\mathrm{R}^{2}(\mathrm{ADJ})$ and $\mathrm{R}^{2}$ values, except for the CVS-II.1, CVS-II.2, WV87, and WV88 data sets. For the first three of these data sets, the FEH model fits the data very poorly, so poor validation performance (including negative $\mathrm{R}^{2}$ (PRESS) values for the CVS-II. 1 and CVS-II. 2 data sets) is not surprising. For the WV88 data set, the drop from $R^{2}$ and $R^{2}(A D J)$ to $R^{2}(P R E S S)$ is not as large, and the FEH model fits better than for the CVS-II.1, CVS-II.2, and WV87 data sets.

The s statistics in Table 3 are generally much larger than the $0.06-0.14$ range suggested in Section 5 for subjectively assessing model lack-of-fit. The WV87 and WV88 data sets are exceptions, where the $s$ values are only slightly larger than the $0.06-0.14$ range. In general, the FEH models appear to have significant lack-of-fits for all of the data sets.

Predicted versus measured plots (see start of Appendix $C$ for a discussion of such plots) for the FEH models are given in the odd numbered figures in Appendix C (i.e., Figures C.1, C.3, ... C.23). For the CVS-II.1, CVS-II.2, and WV-87 data sets, the FEH models yield roughly constant predictions that do not correlate with the variation in measured PCT normalized $B$ releases. For the CVS-I, CVS-II.3, CVS(all), Ramsey, and WVDP data sets, the FEH models tend (in varying degrees) to overpredict lower PCT normalized B releases and underpredict higher releases. For the CVS-II. 4 data set, the FEH model has a slight tendency to overpredict smaller and larger $B$ releases and underpredict intermediate B releases. For the WV88 data set, the FEH model does not appear to be significantly biased, but has considerable uncertainty in predicted $B$ releases. For the SRTC homogeneous data, both the 137-observation and 118-observation FEH models have biased prediction behavior below about $1 \mathrm{~g} / \mathrm{m}^{2}$.

\subsection{First-Order Mixture Model Results}

Table 4 shows that the fitted FOM models have $R^{2}$ values between 0.694 and 0.980 [i.e., the models account for between $69.4 \%$ and $98.0 \%$ of the variation in $\ln (\mathrm{NR})$ values in the various data sets]. Significant decreases in $\mathrm{R}^{2}$ (ADJ) compared to $\mathrm{R}^{2}$ occur for the CVS-II.1, CVS-II.2, and WV87 data sets, indicating it may be possible to reduce the numbers of components (terms) in the models for these data sets. Significant decreases in $\mathrm{R}^{2}$ (PRESS) compared to the corresponding $\mathrm{R}^{2}(\mathrm{ADJ})$ and $\mathrm{R}^{2}$ values occur for the CVS-II.1, CVS-II.2, CVS-II.4, WV87, and WV88 data sets, indicating the presence of influential data points. Because all of these data sets resulted from statistically designed experiments, having one or more compositions much different 
from the others is not a potential explanation for influential points. One possible explanation is that data points can be influential for FOM models when the true behavior is really second-order. Another potential explanation exists for the CVS-I, CVS-II.4, WV87, and WV88 data sets, which contain only a few more points than model terms. In such cases, many data points can be influential due to the small size of the data set. The fact that influential data points exist for a given data set and model may or may not adversely affect the model's predictive ability.

The s statistics in Table 4 for the FOM models are generally larger than the $0.06-0.14$ range suggested in Section 5 for subjectively assessing lack-of-fit of models. The WV87 and WV88 data sets are exceptions, where the s values are within the $0.06-0.14$ range. This is likely due to the relatively small composition regions and narrow ranges of PCT normalized B releases for the WV87 and WV88 data sets. FOM models fitted to such data often perform extremely well. FOM models appear to have slight to moderate lack-of-fits for the other data sets, although not as large as the lack-of-fits for the FEH models. Such lack-of-fits are likely due to the presence of curvilinear and interactive effects of glass components on $\ln$ (PCT B normalized release).

Predicted versus measured plots (see start of Appendix $C$ for a discussion of such plots) for the FOM models are given in the even numbered figures in Appendix C (i.e., Figures C.2, C.4, .. C.24). The FOM models do not show any significant prediction bias for any of the data sets, although there are indications of possible slight biases for certain data sets. The FOM models for the various CVS data sets show a slight tendency to underpredict very low PCT normalized B releases $\left(<0.2 \mathrm{~g} / \mathrm{m}^{2}\right)$. The model for the Ramsey data shows a possible tendency to underpredict larger B releases $\left(>30-35 \mathrm{~g} / \mathrm{m}^{2}\right)$. The uncertainty in model predictions (represented by the scatter of the points about the $45^{\circ}$ line in the predicted versus measured plots) appears to be quite small for all data sets except CVS-II.2, CVS(all), and WVDP, for which moderate uncertainty is seen. Also worth noting is that the FOM model for the WVDP data significantly overpredicts the B release for data point \#11 (Ratio5 glass). This point was not greatly influential, and so was retained for use in developing the models.

\subsection{Second-Order Mixture Model Results}

The reduced second-order mixture models fitted to the WVDP, CVS(all), and Ramsey data sets have $R^{2}$ values that are noticeably larger than the $R^{2}$ values corresponding to the FOM models (see Tables 4 and 6 ). The increase in $R^{2}$ was from 0.842 to 0.923 for the WVDP data, from 0.810 to 0.892 for the CVS(all) data, and from 0.880 to 0.931 for the Ramsey data. For all three data sets, the SOM model $\mathrm{R}^{2}(\mathrm{ADJ})$ and $\mathrm{R}^{2}(\mathrm{PRESS})$ values are typical amounts below the corresponding $\mathrm{R}^{2}$ values, indicating that the reduced-second order models do not appear to overfit the data and that there are no highly influential data points. Hrma, Piepel, et al. ${ }^{3}$ and subsequent unpublished PNL work have shown that indeed there are second-order component effects in the CVS durability data. Other work in the waste glass community has also demonstrated curvilinear and interactive effects of glass components on glass durability.

Although the second-order mixture models improve on the FOM models, the s statistics ( 0.358 for the WVDP data, 0.523 for the CVS(all) data, and 0.504 for the Ramsey data) indicate 
that they still may have some lack of fit. However, this lack of fit may not be practically significant depending on how the models might be used.

Predicted versus measured plots are given in Figures C.25, C.26, and C.27 for the reduced second-order mixture models fitted to the WVDP, CVS(all), and Ramsey data sets. For the WVDP data, comparing Figure C.25 to the corresponding plot for the FOM model in Figure C.20 shows that the reduced second-order model has better precision. For the CVS(all) data, comparing Figure C.26 to the corresponding plot for the FOM model in Figure C.12 shows that the tendency for biased predictions at smaller and larger B release values was slightly reduced, and that precision is somewhat better. For the Ramsey data, comparing Figure C.27 to the corresponding plot for the FOM model in Figure C.14 shows that the second-order model has corrected the tendency toward underpredicting larger B releases.

\subsection{Comparing Goodness-of-Fits of Mixture and Free Energy of Hydration Models}

The FEH and FOM models are first compared by evaluating their goodness-of-fit statistics $\left[R^{2}, R^{2}(A D J)\right.$, and $\left.s\right]$ and the predictive validation statistics $\left[R^{2}(P R E S S)\right]$ listed in Table 4. Comparing these statistics shows that the FOM model fits and predicts better (usually substantially better) than the FEH model for all data sets. This should not be surprising, given the Section 3 result that the FEH model is a restricted form of the FOM model. The FEH approach assumes that the effects of glass components on durability are adequately represented by component FEH values (all adjusted by the same slope " $b$ " and intercept "a" estimated from each data set), whereas the FOM approach lets each data set indicate the effect of each component. For each data set where the FOM model performs much better than the FEH model, the implication is that the component FEH values do not adequately represent the effects of the components on durability over the composition region covered by the data set. Table 4 shows that the FOM component coefficients vary from data set to data set, which implies that the relative effects of components depend on the composition region covered by the data set. The FEH model approach considered in this report does not allow for the relative effects of components to depend on the composition region.

Prédicted versus measured plots (Figures C.1 to C.24 in Appendix C) are also used to compare the performance of FEH and FOM models. The plots for the FEH and FOM models for a particular data set are given on the same page in Appendix $C$ to facilitate their comparison. FOM model predictions for the various data sets are generally free from bias, whereas FEH model predictions are much more prone to bias. FOM model predictions are generally much more precise than FEH model predictions, evidenced by the tighter scatter of points about the $45^{\circ}$ lines in Figures C.2, C.4, ..., C.22, and C.24 compared to Figures C.1, C.3, ..., C.21, and C.23.

The predicted versus measured plots for the reduced SOM models fitted to the WVDP, CVS(all), and Ramsey data sets given in Figures C.25, C.26, and C.27 improve slightly over the corresponding FOM plots, which in turn are better than the corresponding FEH plots.

The ideal basis to compare the performance of the FEH, FOM, and reduced SOM models would be to have some additional data for each data set that were not used to develop the models. 
However, this is not possible, since all data in each data set were used to develop the models. Data from other data sets in this study that fall in the composition region for a given data set could be used to validate the fitted models, but doing so was beyond the scope of this effort. Additional cross-validation approaches (besides the leave-each-data-point-out PRESS approach) could also be used to validate the models, but again these were beyond the scope of this effort. However, Hrma, Piepel, et al. ${ }^{3}$ (Section 12.8) used CVS-II Phase 3 data to validate FOM and reduced SOM models (for PCT normalized B release) fitted to CVS data through CVS-II Phase 2. The mixture models accurately predicted PCT normalized B releases within the uncertainty of the model predictions for nearly all the CVS-II Phase 3 validation data points. FOM and SOM models fitted to CVS data through CVS-II Phase 3 were validated using CVS-II Phase 4 data, and the results (although not published) were again very good. These very good validation performances indicate that FOM and SOM models can be very useful prediction tools.

\subsection{Comparing FEH and FOM Model Coefficients}

The FEH and FOM models fitted to the various data sets are written in the same mathematical form in Table 4, which allows directly comparing their coefficients. Coefficients of models written in the FOM model form do not directly estimate the effects of components on $\ln (\mathrm{NR})$, but can be used to estimate such effects. The effect of a given component is strongly determined by its FOM coefficient. However, it's effect is also determined by the component's value at the glass composition at which the effect is to be estimated, and the coefficients (and effects) of other components. Still, comparing FEH and FOM coefficients in Table 4 can indicate where the FEH theory and assumptions seem to hold, and where they do not. The standard deviations of the coefficients provided in Table 4 must be considered when comparing FEH and FOM coefficients. Differences between FEH and FOM model coefficients are only mentioned in the following discussion when they are significant after accounting for the uncertainties in the coefficients.

$\mathrm{Al}_{2} \mathrm{O}_{3}$ coefficients for $\mathrm{FEH}$ and $\mathrm{FOM}$ models agree fairly well for all data sets except the CVS data sets, where the $\mathrm{Al}_{2} \mathrm{O}_{3}$ coefficients are more negative in the FOM models than in the corresponding FEH models. The reason for this is not clear, but it could be due to the CVS data covering a wider range of compositions than the other data sets.

$\mathrm{B}_{2} \mathrm{O}_{3}$ coefficients are more positive for FOM models than FEH models for all of the data sets. This may indicate a need to reassess the $\mathrm{FEH}$ theory and assumptions for $\mathrm{B}_{2} \mathrm{O}_{3}$.

For all the CVS data sets except CVS-II Phase 1 (where $\mathrm{CaO}$ was varied a small amount in only one glass) and also the Ramsey data set, $\mathrm{CaO}$ coefficients are negative for FOM models but positive for $\mathrm{FEH}$ models. $\mathrm{CaO}$ was combined with $\mathrm{BaO}$ and $\mathrm{MgO}$ in the WV87 and WV88 data sets, so nothing specific can be said about $\mathrm{CaO}$ for those data sets. Neither can anything be said about the WVDP data set, where $\mathrm{CaO}$ was not studied. For the SRTC 137, 118, and 111 data sets, the $\mathrm{CaO}$ FOM model coefficient changes significantly as outlying data points are deleted. This is due to the effect of the outlying data points on correlations of $\mathrm{CaO}$ with other components, and also on the changes in the range of $\mathrm{CaO}$ values (see Table 2). However, with or 
without the outlying data, the $\mathrm{CaO}$ coefficients in the $\mathrm{FOM}$ models have large enough uncertainties that they are not clearly different from the $\mathrm{CaO}$ coefficients in the $\mathrm{FEH}$ models.

$\mathrm{Fe}_{2} \mathrm{O}_{3}$ coefficients in the FEH and FOM models in Table 4 agree very well within their uncertainties except possibly for the Ramsey data set, where the coefficient in the FOM model is more negative than in the FEH model. The $\mathrm{Fe}_{2} \mathrm{O}_{3}$ coefficient in the FOM model for the CVS-II Phase 1 data set is positive, whereas for the rest of the CVS data sets and all other data sets it is negative. However, the standard deviation of the $\mathrm{Fe}_{2} \mathrm{O}_{3} \mathrm{FOM}$ model coefficient is relatively large for the CVS-II Phase 1 data set, and thus the difference from FEH coefficient is not significant. The CVS-II Phase 1 study investigated a shrunken version of the CVS-I glass composition region, where additional constraints were imposed on glass composition (directly via component ranges, and indirectly via constraints on glass properties). The shrinkage of the composition region reduces the effects of some components, and the small data set size limits the accurate estimation of the reduced component effects.

$\mathrm{Li}_{2} \mathrm{O}$ coefficients in the FEH and FOM models in Table 4 agree reasonably well within their uncertainties for all data sets except possibly CVS-II Phase 2 and Phase 4 . The very poor fit of the FEH model to the CVS-II Phase 2 data $\left(\mathrm{R}^{2}=0.113\right)$ is likely the reason for the poor agreement of $\mathrm{Li}_{2} \mathrm{O}$ coefficients for that data set. The reason for the difference in $\mathrm{Li}_{2} \mathrm{O}$ coefficients for the CVS-II Phase 4 data set is not as clear, since the FEH model fits that data reasonably well $\left(R^{2}=0.741\right)$. The four data points in CVS-II Phase 4 in which $\mathrm{Li}_{2} \mathrm{O}$ was varied (see Section 4.1) show an almost perfect linear relationship between $\ln (\mathrm{NR})$ and $\mathrm{Li}_{2} \mathrm{O}$ mole fraction value, and the FOM model $\mathrm{Li}_{2} \mathrm{O}$ coefficient in Table 4 captures that relationship almost perfectly. Hence, the problem would appear to be with the $\mathrm{Li}_{2} \mathrm{O}$ coefficient in the $\mathrm{FEH}$ model.

For the CVS data sets, the $\mathrm{MgO}$ coefficients for the FOM models are generally larger than for the FEH models, but the differences are not significant in all cases. The MgO coefficients in the FEH models for the WV87 and WV88 data sets have essentially identical small negative values, but in the FOM models the MgO coefficient is more negative for the WV87 data set, and positive for the WV88 data set. However, $\mathrm{MgO}+\mathrm{CaO}+\mathrm{BaO}$ were treated as one component in the WV87 and WV88 studies, which is a possible explanation for the differences. Also, the WV88 data set covers a larger composition region than the WV87 data set. $\mathrm{MgO}$ was not studied in the WVDP data set. For the SRTC 137 and SRTC 118 data sets, the MgO FOM model coefficients have relatively large standard deviations, and so the differences with respect to the FEH coefficients aren't significant. The MgO FOM model coefficient for the SRTC 111 data set is larger than the coefficients for the SRTC 137 and 118 data sets, due to the smaller upper limit for $\mathrm{MgO}$. The standard deviation is still relatively large, but the difference from the FEH coefficient is borderline significant.

$\mathrm{Na}_{2} \mathrm{O}$ coefficients in the FEH and FOM models in Table 4 agree very well within their uncertainties for all data sets except CVS-II Phase 2 (where the FOM model coefficient is larger), WVDP (where the FEH model coefficient is larger), and SRTC 118 and SRTC 111 (where the FEH model coefficients are larger). A possible explanation is that the upper limits of $\mathrm{Na}_{2} \mathrm{O}$ for glasses in these data sets are lower than in the other data sets. For the CVS-II Phase 2 data, a likely explanation is the very poor fit of the FEH model $\left(\mathrm{R}^{2}=0.113\right)$. 
$\mathrm{SiO}_{2}$ coefficients in the FEH and FOM models in Table 4 agree very well within their uncertainties for all data sets except possibly the SRTC 137, 118, and 111 data sets. For these SRTC data sets, the $\mathrm{SiO}_{2}$ coefficients for the $\mathrm{FEH}$ models are more negative than the coefficients for the FOM models. The reasons for this are not apparent.

$\mathrm{ZrO}_{2}$ coefficients for FOM models were more negative than for FEH models for the CVS-I, CVS-II Phase 2, CVS-II Phase 3, CVS(all), and WVDP data sets, although the differences were only significant (when considering uncertainties) for the CVS-II Phase 2, CVS-II Phase 3, and CVS(all) data sets. $\mathrm{ZrO}_{2}$ appears to have no effect on $\ln (\mathrm{NR})$ over the composition region covered by the CVS-II Phase 1 data set, which causes the FOM model to have a small $\mathrm{ZrO}_{2}$ coefficient with a large standard deviation. $\mathrm{ZrO}_{2}$ was not studied in the CVS-II Phase 4, Ramsey, WV87, WV88, and SRTC data sets.

$\mathrm{K}_{2} \mathrm{O}$ was only studied in the WV87, WV88, WVDP, and SRTC data sets. In the WV87, WV88, and WVDP data sets, the $\mathrm{K}_{2} \mathrm{O}$ coefficients were more positive for the $\mathrm{FEH}$ models than the FOM models, although the differences are not significant when accounting for the uncertainties in the coefficients. For the SRTC data, the $\mathrm{K}_{2} \mathrm{O}$ coefficients for the FOM models are negative, while the coefficients for the FEH models are positive. $\mathrm{K}_{2} \mathrm{O}$, like other alkalis, is expected to increase PCT B release, so the negative $\mathrm{K}_{2} \mathrm{O}$ coefficients for the FOM models is bothersome. However, the shortcomings of the SRTC data sets discussed in Section 4.5 are possible reasons. In particular, Figures B.11and B.12 show that the coverage of combinations of $\mathrm{K}_{2} \mathrm{O}$ with other components is poor, and this can have significant effects on mixture model coefficients.

FeO was only studied in the Ramsey and SRTC data sets. The FeO coefficients were significantly more positive for the FOM models than the FEH models. This was especially true for the Ramsey and SRTC 111 data sets, which had smaller upper limits for FeO than the SRTC 137 and SRTC 118 data sets. These observations may indicate a need to reassess the FEH theory and assumptions for $\mathrm{FeO}$.

$\mathrm{ThO}_{2}$ was only studied in the WV87, WV88, and WVDP data sets. For the WV87 and WV88 data sets, the $\mathrm{ThO}_{2}$ coefficients in the FOM models are positive, where the coefficients in the FEH models are negative. For the WVDP data set, the FOM and FEH model coefficients for $\mathrm{ThO}_{2}$ are both negative, but not significantly different due to a large standard deviation of the FOM model coefficient. Other work not reported here indicates that $\mathrm{ThO}_{2}$ appears to have no effect on $\ln (\mathrm{NR})$ over the composition region studied in the WVDP data set. These results suggest a possible need to reassess the $\mathrm{FEH}$ theory and assumptions for $\mathrm{ThO}_{2}$.

MnO was only studied in the WV87, WV88, and SRTC data sets. Agreement between $\mathrm{MnO}$ coefficients in the FOM and FEH models is relatively good when accounting for uncertainty, except for the SRTC 111 data set. The MnO coefficient for the SRTC 111 data is noticeably different than for the SRTC 118 data, due to deleting two outlying data points with low $\mathrm{MnO}$ and high $\mathrm{MgO}$ values. 
$\mathrm{P}_{2} \mathrm{O}_{5}$ and $\mathrm{U}_{3} \mathrm{O}_{8}$ were only studied in the WV87 and WV88 data sets, and the small composition regions and ranges of PCT NR releases in these data sets limit any conclusions. . Hence, the results for these components are not discussed.

$\mathrm{Cr}_{2} \mathrm{O}_{3}, \mathrm{CuO}, \mathrm{NiO}$, and $\mathrm{TiO}_{2}$ were only studied in the SRTC data. The FOM model coefficients of $\mathrm{Cr}_{2} \mathrm{O}_{3}, \mathrm{CuO}$, and $\mathrm{NiO}$ change dramatically as the glasses with outlying values of these components are deleted going from SRTC 137 to SRTC 118 to SRTC 111. This is due to: (1) the narrowing of the ranges of these components, and (2) the "removal" of strong pairwise correlations among these and other components caused by the outlying points. The coefficients of $\mathrm{Cr}_{2} \mathrm{O}_{3}$ and $\mathrm{CuO}$ are large due their very small upper limits (see Table 2). Overall, the shortcomings of the SRTC data set limit the ability to make meaningful conclusions for $\mathrm{Cr}_{2} \mathrm{O}_{3}$, $\mathrm{CuO}$, and NiO. The $\mathrm{TiO}_{2}$ coefficients are positive and large for the FOM models (for the SRTC 137,118 , and 111 data sets) compared to the $\mathrm{FEH}$ models. The range of $\mathrm{TiO}_{2}$ values in these glasses is small, and $\mathrm{TiO}_{2}$ is somewhat correlated with certain other components (see Figures B.11 and B.12). This could cause the differences observed, but reassessing the FEH theory and assumptions for $\mathrm{TiO}_{2}$ may be warranted.

The above comparisons show that FOM and FEH model coefficients agree in some cases and disagree in others, depending on the component and data set. For most components, the FOM and FEH model coefficients differed significantly for at least some of the data sets. This confirms that the "preliminary" FEH approach is not flexible enough to adapt to components having different effects for different data sets (and glass composition regions). 



\section{Conclusions}

The free energy of hydration model considered in this report is a restricted form of the firstorder mixture model, and thus does not have the flexibility to fit data sets as well as first- or second-order mixture models. The free energy of hydration approach is "restricted" by the assumption that the effects of glass components on durability are determined primarily by constant values of free energy of hydration for each component, which are applicable to all glass composition regions.

Work in the waste glass field has shown that glass components can have curvilinear and interactive effects on glass durability (resistance to dissolution in water). In other words, the effect of a glass component on durability can depend on the fraction of that component in the glass as well as on the fractions of other components in the glass. Neither the FEH nor FOM modeling approaches (as discussed in this report) can account for curvilinear or interactive effects of glass components (without fitting separate models for different composition regions or subregions). However, second-order and higher-order Scheffé mixture models (and other mixture model forms ${ }^{2}$ ) can directly account for curvilinear or interactive effects of glass components. The FEH approach discussed in this report is referred to as "preliminary FEH" by Jantzen et al. ${ }^{5}$, and they also discuss "final FEH" which adjusts the preliminary FEH in some cases. These adjustments appear to provide some ability to account for curvilinear and interactive effects of glass components, but we have insufficient experience with the "final FEH" approach to comment further.

A properly-developed mixture model has a distinct advantage over a FEH model (of the type discussed in this report) when the goal is to predict PCT normalized releases for glass compositions within the same composition region used to fit the model. By properly-developed we mean that: (a) the data used to develop the mixture model should adequately cover the composition region of interest and should avoid unnecessary correlations among glass component mole (or mass) fractions, and (b) proper statistical approaches should be applied to fit the model, detect outliers or influential data points, detect model lack-of-fit, and validate performance of the model with data not used to fit it. If data to develop a mixture model are limited (in number, or due to poor coverage of the region of interest), or are available only for a composition region different than the one for which predictions are desired, then the FEH approach (or some other theoretical/structural approach) might (or might not) be preferable to a mixture approach. However, the FEH approach still requires fitting its model to data, and shortcomings in the data may also affect the performance of the FEH approach. The performance of the FEH approach also appears to depend on the composition region to which it is applied, and hence extrapolation may not be any more advisable than for the mixture approach.

In conclusion, the mixture approach is recommended over the $\mathrm{FEH}$ approach for predicting PCT releases of glass compositions within the same composition region as the data base available for modeling. 



\section{References}

${ }^{1}$ C.M. Jantzen, N.E. Bibler, D.C. Beam, W.G. Ramsey, and B.J. Waters. Nuclear Waste Product Consistency Test (PCT)--Version 5, WSRC-90-539, Rev. 2, Westinghouse Savannah River Company, Aiken, South Carolina (1992).

2 J.A. Cornell. Experiments with Mixtures: Design, Models, and the Analysis of Mixture Data, Second Edition, John Wiley and Sons, New York (1990).

${ }^{3}$ P.R. Hrma, G.F. Piepel, M.J. Schweiger, D.E. Smith, D.S. Kim, P.E. Redgate, J.D. Vienna, C.A. LoPresti, D.B. Simpson, D.K. Peeler, and M.H. Langowski. Property/Composition Relationships for Hanford High-Level Waste Glasses Melting at $1150^{\circ} \mathrm{C}$, PNL-10359, Volumes 1 and 2, Pacific Northwest Laboratory, Richland, Washington (1994).

${ }^{4}$ P.E. Redgate, G.F. Piepel, and P.R. Hrma. "Second-Order Model Selection in Mixture Experiments", 1992 Proceedings of the Section on Physical and Engineering Sciences, 104-110, American Statistical Association, Alexandria, Virginia (1992).

${ }^{5}$ C.M. Jantzen, J.B. Pickett, K.G. Brown, T.B. Edwards, and D.C. Beam. Technical report not publicly available or referenceable at the time this report was completed, Westinghouse Savannah River Company, Aiken, South Carolina (1995).

${ }^{6}$ D. Öksoy, L.D. Pye, D.F. Bickford, and W.G. Ramsey. "Canonical Correlation of Waste Glass Compositions and Durability, Including $\mathrm{pH}$ ", in Environmental and Waste Management Issues in the Ceramic Industry, Ceramic Transactions, 39, 365-380, American Ceramic Society (1994).

${ }^{7}$ Reimus, M.A.H., G.F. Piepel, G.B. Mellinger, and L.R. Bunnell. West Valley Glass Product Qualification Durability Studies, FY 1987-1988: Effects of Composition, Redox State, Thermal History, and Groundwater, PNL-6723, Pacific Northwest Laboratory, Richland, Washington (1988).

${ }^{\prime}$ K.M. Olson, G.F. Piepel, S.C. Marschman, and G.K. Whiting. Product Consistency Testing of West Valley Compositional Variation Glasses, PNL-10191, Pacific Northwest Laboratory, Richland, Washington (1994).

9 West Valley Nuclear Services. West Valley Demonstration Project Waste Form Qualification Report, WVDP-186, Section 1.3, Rev. 1, West Valley Nuclear Services, West Valley, New York (1995).

${ }^{10}$ N.R. Draper and H. Smith. Applied Regression Analysis, Second Edition, John Wiley and Sons, New York (1981). 



\section{Appendix A}

\section{Data Sets-Glass Compositions and PCT Normalized Releases}

This appendix contains the glass compositions (mole fractions) and PCT normalized elemental releases for the data sets used in this report. Normalized releases for several elements are given in the tables, but only B normalized releases were used in this report.

In several of the references from which the data were taken, compositions were given in terms of weight percents (or weight fractions). In those cases, mole fraction compositions were computed and are given in the tables of this appendix. Also, in several of the references, PCT unnormalized releases or normalized releases that did not normalize for the surface area to volume ratio were given. In those cases, normalized releases in units of $\mathrm{g} / \mathrm{m}^{2}$ were computed and are given in the tables of this appendix. 
Table A.1. CVS-I Glass Compositions (mole fractions) and PCT Normalized B, Li, and Na Releases $\left(\mathrm{g} / \mathrm{m}^{2}\right)$

\begin{tabular}{|c|c|c|c|c|c|c|c|c|}
\hline Comp. & CVS1-1* & CVS1-2 & CVS1-3 & CVS1-4 & CVS1-5 & CVS1-6 & CVS1-7 & CVS1-8 \\
\hline $\mathrm{SiO}_{2}$ & 0.512371 & 0.555060 & 0.419394 & 0.567939 & 0.612381 & 0.477042 & 0.622724 & 0.595322 \\
\hline & 0.105186 & 0.043550 & 0.172363 & 0.171988 & 0.046361 & 0.187143 & 0.047144 & 0.047902 \\
\hline $\mathrm{Na}_{2} \mathrm{O}$ & 0.103769 & 0.048917 & 0.048401 & 0.086933 & 0.072905 & 0.052552 & 0.102097 & 0.090071 \\
\hline $\mathrm{Li}_{2} \mathrm{O}$ & 0.080686 & 0.142048 & 0.140550 & 0.020035 & 0.151218 & 0.152603 & 0.021967 & 0.022321 \\
\hline $\mathrm{CaO}$ & 0.031444 & 0.108127 & 0.000000 & 0.021351 & 0.000000 & 0.000000 & 0.117051 & 0.000000 \\
\hline MgO & 0.057752 & 0.000000 & 0.119089 & 0.118829 & 0.000000 & 0.000000 & 0.000000 & 0.132386 \\
\hline $\mathrm{Fe}_{2} \mathrm{O}_{3}$ & 0.022807 & 0.007594 & 0.007514 & 0.007498 & 0.060634 & 0.008158 & 0.013811 & 0.062648 \\
\hline $\mathrm{Al}_{2} \mathrm{O}_{3}$ & 0.039998 & 0.089206 & 0.082381 & 0.000000 & 0.050648 & 0.000000 & 0.000000 & 0.000000 \\
\hline $\mathrm{ZrO}_{2}$ & 0.022325 & 0.000000 & 0.004869 & 0.000000 & 0.000000 & 0.063440 & 0.069254 & 0.043303 \\
\hline $\mathrm{Ag}_{2} \mathrm{O}$ & 0.000000 & 0.000000 & 0.000000 & 0.000000 & 0.000000 & 0.000000 & 0.000000 & 0.000000 \\
\hline $\mathrm{As}_{2} \mathrm{O}_{3}$ & 0.000000 & 0.000000 & 0.000000 & 0.000000 & 0.000000 & 0.000000 & 0.000000 & 0.000000 \\
\hline $\mathrm{BaO}$ & 0.000305 & 0.000071 & 0.000070 & 0.000070 & 0.000075 & 0.000760 & 0.000077 & 0.000078 \\
\hline CdO & 0.002729 & 0.000634 & 0.000627 & 0.000626 & 0.000675 & 0.006813 & 0.000687 & 0.000698 \\
\hline $\mathrm{Ce}_{2} \mathrm{O}_{3}$ & 0.000204 & 0.000047 & 0.000047 & 0.000047 & 0.000050 & 0.000509 & 0.000051 & 0.000052 \\
\hline $\mathrm{COO}$ & 0.000000 & 0.000000 & 0.000000 & 0.000000 & 0.000000 & 0.000000 & 0.000000 & 0.000000 \\
\hline $\mathrm{Cr}_{2} \mathrm{O}_{3}$ & 0.000385 & 0.000089 & 0.000088 & 0.000088 & 0.000095 & 0.000960 & 0.000097 & 0.000098 \\
\hline $\mathrm{Cs}_{2} \mathrm{O}^{\circ}$ & 0.000249 & 0.000058 & 0.000057 & 0.000057 & 0.000062 & 0.000622 & 0.000063 & 0.000064 \\
\hline CuO & 0.000883 & 0.000205 & 0.000203 & 0.000202 & 0.000218 & 0.002203 & 0.000222 & 0.000226 \\
\hline $\mathbf{F}$ & 0.007376 & 0.001714 & 0.001696 & 0.001692 & 0.001824 & 0.018411 & 0.001855 & 0.001885 \\
\hline $\mathrm{K}_{2} \mathrm{O}$ & 0.000000 & 0.000000 & 0.000000 & 0.000000 & 0.000000 & 0.000000 & 0.000000 & 0.000000 \\
\hline $\mathrm{La}_{2} \mathrm{O}_{3}$ & 0.000896 & 0.000208 & 0.000206 & 0.000206 & 0.000222 & 0.002237 & 0.000225 & 0.000229 \\
\hline $\mathrm{MnO}$ & 0.000808 & 0.000188 & 0.000186 & 0.000185 & 0.000200 & 0.002016 & 0.000203 & 0.000206 \\
\hline $\mathrm{MoO}_{3}$ & 0.000974 & 0.000226 & 0.000224 & 0.000223 & 0.000241 & 0.002430 & 0.000245 & 0.000249 \\
\hline $\mathrm{Nd}_{2} \mathrm{O}_{3}$ & 0.001713 & 0.000398 & 0.000394 & 0.000393 & 0.000424 & 0.004277 & 0.000431 & 0.000438 \\
\hline $\mathrm{NiO}$ & 0.003599 & 0.000836 & 0.000827 & 0.000825 & 0.000890 & 0.008982 & 0.000905 & 0.000920 \\
\hline $\mathrm{P}_{2} \mathrm{O}_{3}$ & 0.000329 & 0.000076 & 0.000076 & 0.000075 & 0.000081 & 0.000821 & 0.000083 & 0.000084 \\
\hline PbO & 0.000000 & 0.000000 & 0.000000 & 0.000000 & 0.000000 & 0.000000 & 0.000000 & 0.000000 \\
\hline PdO & 0.000192 & 0.000045 & 0.000044 & 0.000044 & 0.000047 & 0.000479 & 0.000048 & 0.000049 \\
\hline $\mathrm{Pr}_{8} \mathrm{O}_{11}$ & 0.000046 & 0.000011 & 0.000011 & 0.000010 & 0.000011 & 0.000114 & 0.000012 & 0.000012 \\
\hline $\mathrm{Rb}_{2} \mathrm{O}$ & 0.000126 & 0.000029 & 0.000029 & 0.000029 & 0.000031 & 0.000314 & 0.000032 & 0.000032 \\
\hline $\mathrm{Rh}_{2} \mathrm{O}_{3}$ & 0.000093 & 0.000022 & 0.000021 & 0.000021 & 0.000023 & 0.000231 & 0.000023 & 0.000024 \\
\hline $\mathrm{RuO}_{2}$ & 0.000528 & 0.000123 & 0.000121 & 0.000121 & 0.000130 & 0.001317 & 0.000133 & 0.000135 \\
\hline $\mathrm{SO}_{3}$ & 0.001607 & 0.000373 & 0.000369 & 0.000369 & 0.000398 & 0.004012 & 0.000404 & 0.000411 \\
\hline $\mathrm{Sb}_{2} \mathrm{O}_{3}$ & 0.000000 & 0.000000 & 0.000000 & 0.000000 & 0.000000 & 0.000000 & 0.000000 & 0.000000 \\
\hline $\mathrm{SeO}_{2}$ & 0.000000 & 0.000000 & 0.000000 & 0.000000 & 0.000000 & 0.000000 & 0.000000 & 0.000000 \\
\hline $\mathrm{Sm}_{2} \mathrm{O}_{3}$ & 0.000067 & 0.000016 & 0.000015 & 0.000015 & 0.000017 & 0.000168 & 0.000017 & 0.000017 \\
\hline Sro & 0.000451 & 0.000105 & 0.000104 & 0.000103 & 0.000112 & 0.001125 & 0.000113 & 0.000115 \\
\hline $\mathrm{TeO}_{2}$ & 0.000000 & 0.000000 & 0.000000 & 0.000000 & 0.000000 & 0.000000 & 0.000000 & 0.000000 \\
\hline $\mathrm{TiO}_{2}$ & 0.000000 & 0.000000 & 0.000000 & 0.000000 & 0.000000 & 0.000000 & 0.000000 & 0.000000 \\
\hline $\mathrm{U}_{3} \mathrm{O}_{8}$ & 0.000000 & 0.000000 & 0.000000 & 0.000000 & 0.000000 & 0.000000 & 0.000000 & 0.000000 \\
\hline $\mathrm{WO}_{3}$ & 0.000000 & 0.000000 & 0.000000 & 0.000000 & 0.000000 & 0.000000 & 0.000000 & 0.000000 \\
\hline $\mathrm{Y}_{2} \mathrm{O}_{3}$ & 0.000104 & 0.000024 & 0.000024 & 0.000024 & 0.000026 & 0.000260 & 0.000026 & 0.000027 \\
\hline $\mathrm{ZnO}$ & 0.000000 & 0.000000 & 0.000000 & 0.000000 & 0.000000 & 0.000000 & 0.000000 & 0.000000 \\
\hline РCT B & 0.521 & 0.066 & 0.864 & 20.639 & 0.355 & 6.113 & 0.287 & 1.238 \\
\hline & & 0.154 & 0.791 & 16.935 & 0.462 & 4.892 & 0.331 & 0.744 \\
\hline PCT Na & 0.403 & 0.064 & 0.580 & 17.034 & 0.191 & 3.046 & 0.339 & 0.805 \\
\hline
\end{tabular}

* The glass names are those used in the reference from which the data were taken. 
Table A.1. CVS-I Glass Compositions (mole fractions) and PCT Normalized $\mathrm{B}, \mathrm{Li}$, and $\mathrm{Na}$ Releases $\left(\mathrm{g} / \mathrm{m}^{2}\right)$ (continued)

\begin{tabular}{|c|c|c|c|c|c|c|c|c|}
\hline Comp. & CVS1-9* & CVS1-10 & CVS1-11 & CVS1-12 & CVS1-13 & CVS1-14 & CVS1-15 & CVS1-16 \\
\hline $\mathrm{SiO}_{2}$ & 0.472970 & 0.641699 & 0.473322 & 0.440542 & 0.594718 & 0.475321 & 0.536648 & 0.470582 \\
\hline & 0.190688 & 0.082685 & 0.150660 & 0.159689 & 0.180097 & 0.195347 & 0.041435 & 0.046931 \\
\hline & 0.058733 & 0.103571 & 0.082047 & 0.074839 & 0.188334 & 0.204282 & 0.112723 & 0.197470 \\
\hline $\mathrm{Li}_{2} \mathrm{O}$ & 0.022644 & 0.022637 & 0.022660 & 0.147637 & 0.020980 & 0.022756 & 0.135149 & 0.021868 \\
\hline $\mathrm{CaO}$ & 0.000000 & 0.000000 & 0.120743 & 0.112381 & 0.000000 & 0.000000 & 0.000000 & 0.000000 \\
\hline $\mathrm{MgO}$ & 0.134302 & 0.000000 & 0.000000 & 0.000000 & 0.000000 & 0.000000 & 0.114512 & 0.129702 \\
\hline $\mathrm{Fe}_{2} \mathrm{O}_{3}$ & 0.059318 & 0.008472 & 0.008480 & 0.059198 & 0.007851 & 0.008516 & 0.007225 & 0.035108 \\
\hline $\mathrm{AL}_{2} \mathrm{O}_{3}$ & 0.000000 & 0.079609 & 0.092974 & 0.000000 & 0.002336 & 0.015872 & 0.000000 & 0.092414 \\
\hline $\mathrm{ZrO}_{2}$ & 0.000000 & 0.000000 & 0.000000 & 0.000000 & 0.000000 & 0.071740 & 0.000000 & 0.000000 \\
\hline $\mathrm{Ag}_{2} \mathrm{O}$ & 0.000000 & 0.000000 & 0.000000 & 0.000000 & 0.000000 & 0.000000 & 0.000000 & 0.000000 \\
\hline $\mathrm{As}_{2} \mathrm{O}_{3}$ & 0.000000 & 0.000000 & 0.000000 & 0.000000 & 0.000000 & 0.000000 & 0.000000 & 0.000000 \\
\hline $\mathrm{BaO}$ & 0.000790 & 0.000790 & 0.000632 & 0.000074 & 0.000073 & 0.000079 & 0.000674 & 0.000076 \\
\hline CdO & 0.007077 & 0.007074 & 0.005665 & 0.000659 & 0.000656 & 0.000711 & 0.006034 & 0.000683 \\
\hline $\mathrm{Ce}_{2} \mathrm{O}_{3}$ & 0.000529 & 0.000529 & 0.000423 & 0.000049 & 0.000049 & 0.000053 & 0.000451 & 0.000051 \\
\hline $\mathrm{CoO}$ & 0.000000 & 0.000000 & 0.000000 & 0.000000 & 0.000000 & 0.000000 & 0.000000 & 0.000000 \\
\hline $\mathrm{Cr}_{2} \mathrm{O}_{3}$ & 0.000997 & 0.000997 & 0.000798 & 0.000093 & 0.000092 & 0.000100 & 0.000850 & 0.000096 \\
\hline $\mathrm{Cs}_{2} \mathrm{O}$ & 0.000646 & 0.000646 & $\cdot 0.000517$ & 0.000060 & 0.000060 & 0.000065 & 0.000551 & 0.000062 \\
\hline CuO & 0.002288 & 0.002287 & 0.001832 & 0.000213 & 0.000212 & 0.000230 & 0.001951 & 0.000221 \\
\hline $\mathbf{F}$ & 0.019123 & 0.019118 & 0.015310 & 0.001781 & 0.001772 & 0.001922 & 0.016305 & 0.001847 \\
\hline $\mathrm{K}_{2} \mathrm{O}$ & 0.000000 & 0.000000 & 0.000000 & 0.000000 & 0.000000 & 0.000000 & 0.000000 & 0.000000 \\
\hline $\mathrm{Li}_{2} \mathrm{O}_{3}$ & 0.002324 & 0.002323 & 0.001860 & 0.000216 & 0.000215 & 0.000234 & 0.001981 & 0.000224 \\
\hline Mno & 0.002094 & 0.002093 & 0.001676 & 0.000195 & 0.000194 & 0.000210 & 0.001785 & 0.000202 \\
\hline $\mathrm{MoO}_{3}$ & 0.002524 & 0.002524 & 0.002021 & 0.000235 & 0.000234 & 0.000254 & 0.002152 & 0.000244 \\
\hline $\mathrm{Nd}_{2} \mathrm{O}_{3}$ & 0.004442 & 0.004441 & 0.003556 & 0.000414 & 0.000412 & 0.000446 & 0.003787 & 0.000429 \\
\hline Nió & 0.009330 & 0.009327 & 0.007469 & 0.000869 & 0.000864 & 0.000938 & 0.007955 & 0.000901 \\
\hline $\mathrm{P}_{2} \mathrm{O}_{3}$ & 0.000853 & 0.000853 & 0.000683 & 0.000079 & 0.000079 & 0.000086 & 0.000728 & 0.000082 \\
\hline $\mathrm{PbO}$ & 0.000000 & 0.000000 & 0.000000 & 0.000000 & 0.000000 & 0.000000 & 0.000000 & 0.000000 \\
\hline PdO & 0.000498 & 0.000497 & 0.000398 & 0.000046 & 0.000046 & 0.000050 & 0.000424 & 0.000048 \\
\hline $\mathrm{Pr}_{6} \mathrm{O}_{11}$ & 0.000119 & 0.000119 & 0.000095 & 0.000011 & 0.000011 & 0.000012 & 0.000101 & 0.000011 \\
\hline $\mathrm{Rb}_{2} \mathrm{O}$ & 0.000326 & 0.000326 & 0.000261 & 0.000030 & 0.000030 & 0.000033 & 0.000278 & 0.000031 \\
\hline $\mathrm{Rh}_{2} \mathrm{O}_{3}$ & 0.000240 & 0.000240 & 0.000192 & 0.000022 & 0.000022 & 0.000024 & 0.000205 & 0.000023 \\
\hline $\mathrm{RuO}_{2}$ & 0.001368 & 0.001367 & 0.001095 & 0.000127 & 0.000127 & 0.000137 & 0.001166 & 0.000132 \\
\hline $\mathrm{SO}_{3}$ & 0.004167 & 0.004165 & 0.003336 & 0.000388 & 0.000386 & 0.000419 & 0.003553 & 0.000402 \\
\hline $\mathrm{Sb}_{2} \mathrm{O}_{3}$ & 0.000000 & 0.000000 & 0.000000 & 0.000000 & 0.000000 & 0.000000 & & 0.000000 \\
\hline $\mathrm{SeO}_{2}$ & 0.000000 & 0.000000 & 0.000000 & 0.000000 & 0.000000 & 0.000000 & 0.000000 & 0.000000 \\
\hline $\mathrm{Sm}_{2} \mathrm{O}_{3}$ & 0.000175 & 0.000175 & 0.000140 & 0.000016 & 0.000016 & 0.000018 & 0.000149 & 0.000017 \\
\hline Sro & 0.001169 & 0.001168 & 0.000936 & 0.000109 & & & 0.000997 & 0.000113 \\
\hline $\mathrm{TeO}_{2}$ & 0.000000 & 0.000000 & 0.000000 & 0.000000 & 0.000000 & 0.000000 & 0.000000 & 0.000000 \\
\hline $\mathrm{TiO}_{2}$ & & 0.000000 & 0.000000 & & 0.000000 & 0.000000 & 0.000000 & 0.000000 \\
\hline $\mathrm{U}_{3} \mathrm{O}_{8}$ & 0.000000 & 0.000000 & 0.000000 & 0.000000 & 0.000000 & & 0.000000 & 0.000000 \\
\hline $\mathrm{WO}_{3}$ & 0.000000 & 0.000000 & 0.000000 & 0.000000 & 0.000000 & 0.000000 & 0.000000 & 0.000000 \\
\hline $\mathrm{Y}_{2} \mathrm{O}_{3}$ & 0.000270 & 0.000270 & 0.000216 & 0.000025 & 0.000025 & 0.000027 & 0.000230 & 0.000026 \\
\hline $\mathrm{ZnO}$ & 0.000000 & 0.000000 & 0.000000 & 0.000000 & 0.000000 & 0.000000 & 0.000000 & 0.000000 \\
\hline РCT B & 10.9 & & 0.0 & & & & & \\
\hline PCT $\mathrm{Li}$ & & 0.386 & 0.187 & 4.171 & 12.903 & 8.011 & 11.198 & 0.266 \\
\hline PCT Na & 7.897 & 0.095 & 0.099 & 4.395 & 12.413 & 5.790 & 13.995 & 0.540 \\
\hline
\end{tabular}

* The glass names are those used in the reference from which the data were taken. 
Table A.1. CVS-I Glass Compositions (mole fractions) and PCT Normalized $\mathrm{B}, \mathrm{Li}$, and $\mathrm{Na}$ Releases $\left(\mathrm{g} / \mathrm{m}^{2}\right)$ (continued)

\begin{tabular}{|c|c|c|c|c|c|c|c|}
\hline Comp. & CVS1-17* & CVS1-18 & CVS1-19 & CVS1-20 & CVS1-21 & CVS1-22 & CVS1-23 \\
\hline $\mathrm{SiO}_{2}$ & 0.520558 & 0.429600 & 0.512371 & 0.512371 & 0.567939 & 0.595322 & 0.563543 \\
\hline $\mathrm{B}_{2} \mathrm{O}_{3}$ & 0.049425 & 0.043993 & 0.105186 & 0.105186 & 0.171988 & 0.047902 & 0.090233 \\
\hline $\mathrm{Na}_{2} \mathrm{O}$ & 0.161552 & 0.117211 & 0.103769 & 0.103769 & 0.086933 & 0.090071 & 0.111531 \\
\hline $\mathrm{Li}_{2} \mathrm{O}$ & 0.023030 & 0.143492 & 0.080686 & 0.080686 & 0.020035 & 0.022321 & 0.082463 \\
\hline $\mathrm{CaO}$ & 0.122713 & 0.021845 & 0.031444 & 0.031444 & 0.021351 & 0.000000 & 0.033863 \\
\hline $\mathrm{MgO}$ & 0.000000 & 0.121581 & 0.057752 & 0.057752 & 0.118829 & 0.132386 & 0.013695 \\
\hline $\mathrm{Fe}_{2} \mathrm{O}_{3}$ & 0.060331 & 0.007671 & 0.022807 & 0.022807 & 0.007498 & 0.062648 & 0.048513 \\
\hline $\mathrm{Al}_{2} \mathrm{O}_{3}$ & 0.000000 & 0.000000 & 0.039998 & 0.039998 & 0.000000 & 0.000000 & 0.029387 \\
\hline $\mathrm{ZrO}_{2}$ & 0.000000 & 0.064624 & 0.022325 & 0.022325 & 0.000000 & 0.043303 & 0.003360 \\
\hline $\mathrm{Ag}_{2} \mathrm{O}$ & 0.000000 & 0.000000 & 0.000000 & 0.000000 & 0.000000 & 0.000000 & 0.000000 \\
\hline $\mathrm{As}_{2} \mathrm{O}_{3}$ & 0.000000 & 0.000000 & 0.000000 & 0.000000 & 0.000000 & 0.000000 & 0.000000 \\
\hline $\mathrm{BaO}$ & 0.000803 & 0.000644 & 0.000305 & 0.000305 & 0.000070 & 0.000078 & 0.000301 \\
\hline $\mathrm{CdO}$ & 0.007197 & 0.005766 & 0.002729 & 0.002729 & 0.000626 & 0.000698 & 0.002701 \\
\hline $\mathrm{Ce}_{2} \mathrm{O}_{3}$ & 0.000538 & 0.000431 & 0.000204 & 0.000204 & 0.000047 & 0.000052 & 0.000202 \\
\hline $\mathrm{CoO}$ & 0.000000 & 0.000000 & 0.000000 & 0.000000 & 0.000000 & 0.000000 & 0.000000 \\
\hline $\mathrm{Cr}_{2} \mathrm{O}_{3}$ & 0.001014 & 0.000812 & 0.000385 & 0.000385 & 0.000088 & 0.000098 & 0.000381 \\
\hline $\mathrm{Cs}_{2} \mathrm{O}$ & 0.000657 & 0.000526 & 0.000249 & 0.000249 & 0.000057 & 0.000064 & 0.000246 \\
\hline CuO & 0.002327 & 0.001864 & 0.000883 & 0.000883 & 0.000202 & 0.000226 & 0.000873 \\
\hline $\mathbf{F}$ & 0.019450 & 0.015581 & 0.007376 & 0.007376 & 0.001692 & 0.001885 & 0.007299 \\
\hline $\mathrm{K}_{2} \mathrm{O}$ & 0.000000 & 0.000000 & 0.000000 & 0.000000 & 0.000000 & 0.000000 & 0.000000 \\
\hline $\mathrm{La}_{2} \mathrm{O}_{3}$ & 0.002364 & 0.001893 & 0.000896 & 0.000896 & 0.000206 & 0.000229 & 0.000887 \\
\hline $\mathrm{MnO}$ & 0.002129 & 0.001706 & 0.000808 & 0.000808 & 0.000185 & 0.000206 & 0.000799 \\
\hline $\mathrm{MoO}_{3}$ & 0.002567 & 0.002057 & 0.000974 & 0.000974 & 0.000223 & 0.000249 & 0.000963 \\
\hline $\mathrm{Nd}_{2} \mathrm{O}_{3}$ & 0.004518 & 0.003619 & 0.001713 & 0.001713 & 0.000393 & 0.000438 & 0.001695 \\
\hline $\mathrm{NiO}$ & 0.009489 & 0.007601 & 0.003599 & 0.003599 & 0.000825 & 0.000920 & 0.003561 \\
\hline $\mathrm{P}_{2} \mathrm{O}_{3}$ & 0.000868 & 0.000695 & 0.000329 & 0.000329 & 0.000075 & 0.000084 & 0.000326 \\
\hline $\mathrm{PbO}$ & 0.000000 & 0.000000 & 0.000000 & 0.000000 & 0.000000 & 0.000000 & 0.000000 \\
\hline PdO & 0.000506 & 0.000405 & 0.000192 & 0.000192 & 0.000044 & 0.000049 & 0.000190 \\
\hline $\mathrm{Pr}_{6} \mathrm{O}_{11}$ & 0.000121 & 0.000097 & 0.000046 & 0.000046 & 0.000010 & 0.000012 & 0.000045 \\
\hline $\mathrm{Rb}_{2} \mathrm{O}$ & 0.000331 & 0.000265 & 0.000126 & 0.000126 & 0.000029 & 0.000032 & 0.000124 \\
\hline $\mathrm{Rh}_{2} \mathrm{O}_{3}$ & 0.000244 & 0.000195 & 0.000093 & 0.000093 & 0.000021 & 0.000024 & 0.000092 \\
\hline $\mathrm{RuO}_{2}$ & 0.001391 & 0.001114 & 0.000528 & 0.000528 & 0.000121 & 0.000135 & 0.000522 \\
\hline $\mathrm{SO}_{3}$ & 0.004238 & 0.003395 & 0.001607 & 0.001607 & 0.000369 & 0.000411 & 0.001590 \\
\hline $\mathrm{Sb}_{2} \mathrm{O}_{3}$ & 0.000000 & 0.000000 & 0.000000 & 0.000000 & 0.000000 & 0.000000 & 0.000000 \\
\hline $\mathrm{SeO}_{2}$ & 0.000000 & 0.000000 & 0.000000 & 0.000000 & 0.000000 & 0.000000 & 0.000000 \\
\hline $\mathrm{Sm}_{2} \mathrm{O}_{3}$ & 0.000178 & 0.000142 & 0.000067 & 0.000067 & 0.000015 & 0.000017 & 0.000067 \\
\hline Sró & 0.001189 & 0.000952 & 0.000451 & 0.000451 & 0.000103 & 0.000115 & 0.000446 \\
\hline $\mathrm{TeO}_{2}$ & 0.000000 & 0.000000 & 0.000000 & 0.000000 & 0.000000 & 0.000000 & 0.000000 \\
\hline $\mathrm{TiO}_{2}^{-}$ & 0.000000 & 0.000000 & 0.000000 & 0.000000 & 0.000000 & 0.000000 & 0.000000 \\
\hline $\mathrm{U}_{3} \mathrm{O}_{8}$ & 0.000000 & 0.000000 & 0.000000 & 0.000000 & 0.000000 & 0.000000 & 0.000000 \\
\hline $\mathrm{WO}_{3}$ & 0.000000 & 0.000000 & 0.000000 & 0.000000 & 0.000000 & 0.000000 & 0.000000 \\
\hline $\mathrm{Y}_{2} \mathrm{O}_{3}$ & 0.000274 & 0.000220 & 0.000104 & 0.000104 & 0.000024 & 0.000027 & 0.000103 \\
\hline $\mathrm{ZnO}$ & 0.000000 & 0.000000 & 0.000000 & 0.000000 & 0.000000 & 0.000000 & 0.000000 \\
\hline PCT B & 2.235 & 11.238 & 0.523 & 0.455 & 18.850 & 1.119 & 0.525 \\
\hline PCT Li & 2.075 & 8.065 & 0.533 & 0.468 & 15.591 & 0.704 & 0.535 \\
\hline $\mathrm{PCT} \mathrm{Na}$ & 2.304 & 8.040 & 0.433 & 0.396 & 15.536 & 0.760 & 0.487 \\
\hline
\end{tabular}

* The glass names are those used in the reference from which the data were taken. 
Table A.2. CVS-II Phase 1 Glass Compositions (mole fractions) and PCT Normalized $\mathrm{B}, \mathrm{Li}$, and Na Releases $\left(\mathrm{g} / \mathrm{m}^{2}\right)$

\begin{tabular}{|c|c|c|c|c|c|c|c|c|}
\hline Comp. & CVS2-1* & CVS2-2 & CVS2-3 & CVS2-4 & CVS2-5 & CVS2-6 & CVS2-7 & CVS2-8 \\
\hline $\mathrm{SiO}_{2}$ & 0.537807 & 0.526245 & 0.497874 & 0.574264 & 0.528889 & 0.584895 & 0.514167 & 0.532857 \\
\hline$R 0^{2}$ & 0.077626 & 0.063370 & 0.122109 & 0.096912 & 0.152730 & 0.065778 & 0.156707 & 0.062937 \\
\hline $\mathrm{Na}_{2} \mathrm{O}$ & 0.069834 & 0.089788 & 0.072735 & 0.069588 & 0.108974 & 0.073885 & 0.072479 & 0.099475 \\
\hline $\mathrm{Li}_{2} \mathrm{O}$ & 0.124156 & 0.126548 & 0.093753 & 0.110522 & 0.044759 & 0.083631 & 0.126925 & 0.125683 \\
\hline CaO & 0.000000 & 0.078668 & 0.080387 & 0.076909 & 0.041498 & 0.081657 & 0.010757 & 0.000000 \\
\hline MgO & 0.076707 & 0.000000 & 0.015979 & 0.000000 & 0.000000 & 0.007466 & 0.000000 & 0.077650 \\
\hline $\mathrm{Fe}_{2} \mathrm{O}_{3}$ & 0.015488 & 0.017760 & 0.018148 & 0.015433 & 0.016750 & 0.049158 & 0.016074 & 0.044683 \\
\hline $\mathrm{Al}_{2} \mathrm{O}_{3}$ & 0.048515 & 0.067993 & 0.065184 & 0.037406 & 0.052469 & 0.010202 & 0.059981 & 0.037447 \\
\hline $\mathrm{ZrO}_{2}$ & 0.005018 & 0.015344 & 0.019234 & 0.005000 & 0.005427 & 0.005309 & 0.005208 & 0.005080 \\
\hline $\mathrm{Ag}_{2} \mathrm{O}$ & 0.000000 & 0.000000 & 0.000000 & 0.000000 & 0.000000 & 0.000000 & 0.000000 & 0.000000 \\
\hline $\mathrm{As}_{2} \mathrm{O}_{3}$ & 0.000000 & 0.000000 & 0.000000 & 0.000000 & 0.000000 & 0.000000 & 0.000000 & 0.000000 \\
\hline $\mathrm{BaO}$ & 0.000577 & 0.000184 & 0.000188 & 0.000180 & 0.000625 & 0.000490 & 0.000485 & 0.000183 \\
\hline CdO & 0.005173 & 0.001648 & 0.001684 & 0.001611 & 0.005595 & 0.004386 & 0.004349 & 0.001637 \\
\hline $\mathrm{Ce}_{2} \mathrm{O}_{3}$ & 0.000387 & 0.000123 & 0.000126 & 0.000120 & 0.000418 & 0.000328 & 0.000325 & 0.000122 \\
\hline $\mathrm{CoO}$ & 0.000000 & 0.000000 & 0.000000 & 0.000000 & 0.000000 & 0.000000 & 0.000000 & 0.000000 \\
\hline $\mathrm{Cr}_{2} \mathrm{O}_{3}$ & 0.000729 & 0.000232 & 0.000237 & 0.000227 & 0.000788 & 0.000618 & 0.000613 & 0.000231 \\
\hline $\mathrm{Cs}_{2} \mathrm{O}$ & 0.000472 & 0.000150 & 0.000154 & 0.000147 & 0.000511 & 0.000400 & 0.000397 & 0.000149 \\
\hline CuO & 0.001673 & 0.000533 & 0.000544 & 0.000521 & 0.001809 & 0.001418 & 0.001406 & 0.000529 \\
\hline $\mathbf{F}$ & 0.013981 & 0.004453 & 0.004550 & 0.004354 & 0.015120 & 0.011852 & 0.011753 & 0.004423 \\
\hline $\mathrm{K}_{2} \mathrm{O}$ & 0.000000 & 0.000000 & 0.000000 & 0.000000 & 0.000000 & 0.000000 & 0.000000 & 0.000000 \\
\hline $\mathrm{La}_{2} \mathrm{O}_{3}$ & 0.001699 & 0.000541 & 0.000553 & 0.000529 & 0.001837 & 0.001440 & 0.001428 & 0.000537 \\
\hline Mno & 0.001531 & 0.000488 & 0.000498 & 0.000477 & 0.001655 & 0.001298 & 0.001287 & 0.000484 \\
\hline $\mathrm{MoO}_{3}$ & 0.001845 & 0.000588 & 0.000601 & 0.000575 & 1996 & 0.001564 & 1551 & 0.000584 \\
\hline $\mathrm{Nd}_{2} \mathrm{O}_{3}$ & 0.003247 & 0.001034 & 0.001057 & 0.001011 & 0.003512 & 0.002753 & 0.002730 & 0.001027 \\
\hline Niós & 0.006821 & 0.002173 & 0.002220 & 0.002124 & 7377 & 0.0 & 5734 & 0.002158 \\
\hline $\mathrm{P}_{2} \mathrm{O}_{3}$ & 0.000624 & 0.000199 & 0.000203 & 0.000194 & 0.000675 & 0.000529 & 0.000524 & 0.000197 \\
\hline PbO & 0.000000 & 0.000000 & 0.000000 & 0.000000 & 0.000000 & 0.000000 & 0.000000 & 0.000000 \\
\hline PdO & 0.000364 & 0.000116 & 0.000118 & 0.000113 & 0.0 & & 0306 & 20115 \\
\hline $\mathrm{Pr}_{6} \mathrm{O}_{n}$ & 0.000087 & 0.000028 & 0.000028 & 0.000027 & 0.000094 & 0.000073 & 0.000073 & 0.000027 \\
\hline $\mathrm{Rb}_{2} \mathrm{O}$ & 0.000238 & 0.000076 & 0.000078 & 0.000074 & 0.00 & 0.000202 & 0.000200 & 0.000075 \\
\hline $\mathrm{Rh}_{2} \mathrm{O}_{3}$ & 0.000175 & 0.000056 & 0.000057 & 0.000055 & 0.000190 & 0.000149 & 0.000147 & 0.000055 \\
\hline $\mathrm{RuO}_{2}$ & 0.001000 & 0.000319 & 0.000325 & 0.000311 & 0.001081 & 0.000848 & 0.000841 & 0.000316 \\
\hline $\mathrm{SO}_{3}$ & 0.003046 & 0.000970 & 0.000991 & 0.000949 & & & 0.002561 & 00964 \\
\hline $\mathrm{Sb}_{2} \mathrm{O}_{3}$ & 0.000000 & 0.000000 & 0.000000 & 0.000000 & 0.000000 & 0.000000 & 0.000000 & 0.000000 \\
\hline 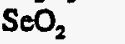 & 0.000000 & 0.000000 & 0.000000 & 0.000000 & 0.000000 & 0.000000 & 0.000000 & 0.000000 \\
\hline $\mathrm{Sm}_{2} \mathrm{O}_{3}$ & 0.000128 & 0.000041 & 0.000042 & 0.000040 & 0.000138 & 0.000108 & 0.000107 & 0.000040 \\
\hline Sró & 0.000855 & 0.000272 & 0.000278 & 0.000266 & 0.000924 & 0.000724 & 0.000718 & 0.000270 \\
\hline $\mathrm{TeO}_{2}$ & 0.000000 & 0.000000 & 0.000000 & 0.000000 & 0.000000 & 0.000000 & 0.000000 & 0.000000 \\
\hline $\mathrm{TiO}_{2}$ & 0.000000 & 0.000000 & 0.000000 & 0.000000 & 0.000000 & 0.000000 & 0.000000 & 0.000000 \\
\hline $\mathrm{U}_{3} \mathrm{O}_{3}$ & 0.000000 & 0.000000 & 0.000000 & 0.000000 & 0.000000 & 0.000000 & 0.000000 & 0.000000 \\
\hline $\mathrm{WO}_{3}$ & 0.000000 & & 0.000000 & 0.000000 & 0.000000 & 0.000000 & 0.000000 & 0.000000 \\
\hline $\mathrm{Y}_{2} \mathrm{O}_{3}$ & 0.000197 & 0.000063 & 0.000064 & 0.000061 & 0.000213 & 0.000167 & 0.000166 & 0.000062 \\
\hline $\mathrm{ZnO}$ & 0.000000 & 0.000000 & 0.000000 & 0.000000 & 0.000000 & 0.000000 & 0.000000 & 0.000000 \\
\hline PCT B & 0.312 & 0.12 & 0.137 & & & & 0.7 & 0.4 \\
\hline & & & & & & & & 0.523 \\
\hline PCT Na & 0.208 & 0.188 & 0.125 & 0.182 & 0.284 & 1.284 & 0.343 & 0.445 \\
\hline
\end{tabular}

* The glass names are those used in the reference from which the data were taken. 
Table A.2. CVS-II Phase 1 Glass Compositions (mole fractions) and PCT Normalized $\mathrm{B}, \mathrm{Li}$, and $\mathrm{Na}$ Releases $\left(\mathrm{g} / \mathrm{m}^{2}\right)$ (continued)

\begin{tabular}{|c|c|c|c|c|c|c|c|c|}
\hline Comp. & CVS2-9* & CVS2-10 & CVS2-11 & CVS2-12 & CVS2-13 & CVS2-14 & CVS2-15 & CVS2-16 \\
\hline $\mathrm{SiO}_{2}$ & 0.582711 & 0.527333 & 0.517212 & 0.491330 & 0.516203 & 0.491918 & 0.517626 & 0.579488 \\
\hline & 0.087399 & 0.164828 & 0.068192 & 0.153935 & 0.063168 & 0.121090 & 0.066128 & 0.098382 \\
\hline $\mathrm{Na}_{2} \mathrm{O}$ & 0.096090 & 0.142232 & 0.084960 & 0.071197 & 0.171503 & 0.083142 & 0.180389 & 0.118063 \\
\hline $\mathrm{Li}_{2} \mathrm{O}$ & 0.129421 & 0.045178 & 0.126931 & 0.098309 & 0.047304 & 0.104503 & 0.047100 & 0.081628 \\
\hline $\mathrm{CaO}$ & 0.000000 & 0.000000 & 0.078906 & 0.078687 & 0.033607 & 0.057287 & 0.070482 & 0.009627 \\
\hline MgO & 0.000000 & 0.000000 & 0.015684 & 0.015641 & 0.077935 & 0.031884 & 0.000000 & 0.013556 \\
\hline $\mathrm{Fe}_{2} \mathrm{O}_{3}$ & 0.028737 & 0.028278 & 0.015834 & 0.015790 & 0.015736 & 0.016094 & 0.016473 & 0.029285 \\
\hline $\mathrm{Al}_{2} \mathrm{O}_{3}$ & 0.008724 & 0.069048 & 0.006200 & 0.055706 & 0.055206 & 0.015313 & 0.048763 & 0.014736 \\
\hline $\mathrm{ZrO}_{2}$ & 0.052309 & 0.005478 & 0.047968 & 0.005116 & 0.005098 & 0.052145 & 0.005337 & 0.020323 \\
\hline $\mathrm{Ag}_{2} \mathrm{O}$ & 0.000000 & 0.000000 & 0.000000 & 0.000000 & 0.000000 & 0.000000 & 0.000000 & 0.000000 \\
\hline $\mathrm{As}_{2} \mathrm{O}_{3}$ & 0.000000 & 0.000000 & 0.000000 & 0.000000 & 0.000000 & 0.000000 & 0.000000 & 0.000000 \\
\hline $\mathrm{BaO}^{\circ}$ & 0.000188 & 0.000227 & 0.000491 & 0.000184 & 0.000183 & 0.000343 & 0.000614 & 0.000450 \\
\hline Cdo & 0.001685 & 0.002033 & 0.004397 & 0.001648 & 0.001643 & 0.003071 & 0.005503 & 0.004027 \\
\hline $\mathrm{Ce}_{2} \mathrm{O}_{3}$ & 0.000126 & 0.000152 & 0.000328 & 0.000123 & 0.000123 & 0.000229 & 0.000411 & 0.000301 \\
\hline $\mathrm{CoO}^{\circ}$ & 0.000000 & 0.000000 & 0.000000 & 0.000000 & 0.000000 & 0.000000 & 0.000000 & 0.000000 \\
\hline $\mathrm{C}_{\tau_{2}} \mathrm{O}_{3}$ & 0.000237 & 0.000287 & 0.000620 & 0.000232 & 0.000231 & 0.000433 & 0.000775 & 0.000567 \\
\hline $\mathrm{Cs}_{2} \mathrm{O}$ & 0.000154 & 0.000186 & 0.000401 & 0.000150 & 0.000150 & 0.000280 & 0.000502 & 0.000368 \\
\hline $\mathrm{CuO}$ & 0.000545 & 0.000657 & 0.001422 & 0.000533 & 0.000531 & 0.000993 & 0.001779 & 0.001302 \\
\hline $\mathrm{F}$ & 0.004554 & 0.005494 & 0.011881 & 0.004454 & 0.004439 & 0.008299 & 0.014870 & 0.010883 \\
\hline $\mathrm{K}_{2} \mathrm{O}$ & 0.000000 & 0.000000 & 0.000000 & 0.000000 & 0.000000 & 0.000000 & 0.000000 & 0.000000 \\
\hline $\mathrm{La}_{2} \mathrm{O}_{3}$ & 0.000553 & 0.000668 & 0.001444 & 0.000541 & 0.000539 & 0.001008 & 0.001807 & 0.001322 \\
\hline $\mathrm{MnO}$ & 0.000499 & 0.000602 & 0.001301 & 0.000488 & 0.000486 & 0.000909 & 0.001628 & 0.001191 \\
\hline $\mathrm{MoO}_{3}$ & 0.000601 & 0.000725 & 0.001568 & 0.000588 & 0.000586 & 0.001095 & 0.001963 & 0.001437 \\
\hline $\mathrm{Nd}_{2} \mathrm{O}_{3}$ & 0.001058 & 0.001276 & 0.002760 & 0.001035 & 0.001031 & 0.001928 & 0.003454 & 0.002528 \\
\hline $\mathrm{NiO}^{\circ}$ & 0.002222 & 0.002680 & 0.005796 & 0.002173 & 0.002166 & 0.004049 & 0.007255 & 0.005309 \\
\hline $\mathrm{P}_{2} \mathrm{O}_{5}$ & 0.000203 & 0.000245 & 0.000530 & 0.000199 & 0.000198 & 0.000370 & 0.000663 & 0.000486 \\
\hline PoO & 0.000000 & 0.000000 & 0.000000 & 0.000000 & 0.000000 & 0.000000 & & 0.000000 \\
\hline PdO & 0.000118 & 0.000143 & 0.000309 & 0.000116 & 0.000115 & 0.000216 & 0.000387 & 0.000283 \\
\hline $\mathrm{Pr}_{8} \mathrm{O}_{11}$ & 0.000028 & 0.000034 & 0.000074 & 0.000028 & 0.000028 & 0.000051 & 0.000092 & 0.000067 \\
\hline $\mathrm{Rb}_{2} \mathrm{O}$ & 0.000078 & 0.000094 & 0.000202 & 0.000076 & 0.000076 & 0.000141 & 0.000253 & 0.000185 \\
\hline $\mathrm{Rh}_{2} \mathrm{O}_{3}$ & 0.000057 & 0.000069 & 0.000149 & 0.000056 & 0.000056 & 0.000104 & 0.000187 & 0.000137 \\
\hline $\mathrm{RuO}_{2}$ & 0.000326 & 0.000393 & 0.000850 & 0.000319 & 0.000317 & 0.000594 & 0.001064 & 0.000778 \\
\hline $\mathrm{SO}_{3}$ & 0.000992 & 0.001197 & 0.002589 & 0.000970 & 0.000967 & 0.001808 & 0.003240 & 0.002371 \\
\hline $\mathrm{Sb}_{2} \mathrm{O}_{3}$ & 0.000000 & 0.000000 & 0.000000 & 0.000000 & 0.000000 & 0.000000 & 0.000000 & 0.000000 \\
\hline $\mathrm{SeO}_{2}$ & 0.000000 & 0.000000 & 0.000000 & 0.000000 & 0.000000 & 0.000000 & 0.000000 & 0.000000 \\
\hline $\mathrm{Sm}_{2} \mathrm{O}_{3}$ & 0.000042 & 0.000050 & 0.000108 & 0.000041 & 0.000041 & 0.000076 & 0.000136 & 0.000099 \\
\hline Sro & 0.000278 & 0.000336 & 0.000726 & 0.000272 & 0.000271 & 0.000507 & 0.000909 & 0.000665 \\
\hline $\mathrm{TeO}_{2}$ & 0.000000 & 0.000000 & 0.000000 & 0.000000 & 0.000000 & 0.000000 & 0.000000 & 0.000000 \\
\hline $\mathrm{TiO}_{2}$ & 0.000000 & 0.000000 & 0.000000 & 0.000000 & 0.000000 & 0.000000 & 0.000000 & 0.000000 \\
\hline $\mathrm{U}_{3} \mathrm{O}_{8}$ & 0.000000 & 0.000000 & 0.000000 & 0.000000 & 0.000000 & 0.000000 & 0.000000 & 0.000000 \\
\hline $\mathrm{WO}_{3}$ & 0.000000 & 0.000000 & 0.000000 & 0.000000 & 0.000000 & 0.000000 & 0.000000 & 0.000000 \\
\hline $\mathrm{Y}_{2} \mathrm{O}_{3}$ & 0.000064 & 0.000077 & 0.000168 & 0.000063 & 0.000063 & 0.000117 & 0.000210 & 0.000153 \\
\hline $\mathrm{ZnO}$ & 0.000000 & 0.000000 & 0.000000 & 0.000000 & 0.000000 & 0.000000 & 0.000000 & 0.000000 \\
\hline РCT B & 0.560 & 1.332 & 1.587 & 0.194 & 0.360 & 1.656 & 0.331 & 2.937 \\
\hline PCT Li & 0.620 & 1.322 & 1.564 & 0.262 & 0.362 & 1.468 & 0.451 & 2.349 \\
\hline PCT Na & 0.374 & 0.828 & 1.370 & 0.184 & 0.478 & 1.268 & 0.608 & 2.182 \\
\hline
\end{tabular}

* The glass names are those used in the reference from which the data were taken. 
Table A.2. C.VS-II Phase 1 Glass Compositions (mole fractions) and PCT Normalized $\mathrm{B}, \mathrm{Li}$, and $\mathrm{Na}$ Releases $\left(\mathrm{g} / \mathrm{m}^{2}\right)$ (continued)

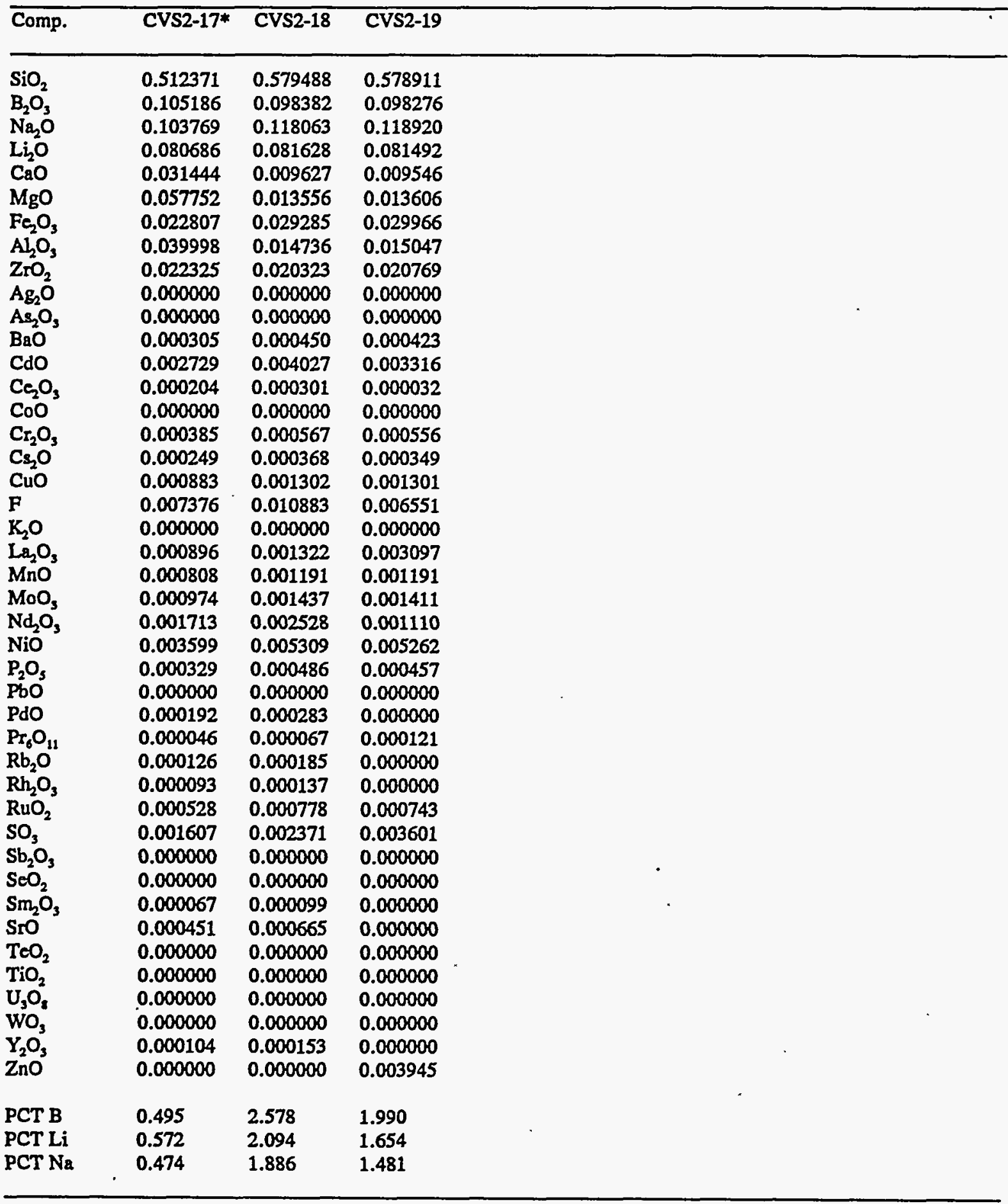

* The glass names are those used in the reference from which the data were taken. 
Table A.3. CVS-II Phase 2 Glass Compositions (mole fractions) and PCT Normalized $\mathrm{B}, \mathrm{Li}$, and $\mathrm{Na}$ Releases $\left(\mathrm{g} / \mathrm{m}^{2}\right)$

\begin{tabular}{|c|c|c|c|c|c|c|c|c|}
\hline Comp. & CVS2-20* & CVS2-21 & CVS2-22 & CVS2-23 & CVS2-24 & CVS2-25 & CVS2-26 & CVS2-27 \\
\hline $\mathrm{SiO}_{2}$ & 0.603222 & 0.545304 & 0.554513 & 0.592645 & 0.471671 & 0.534599 & 0.457421 & 0.550251 \\
\hline $10^{2}$ & 0.045668 & 0.108492 & 0.041980 & 0.046841 & 0.182383 & 0.045601 & 0.178191 & 0.043465 \\
\hline $\mathrm{Na}_{2} \mathrm{O}$ & 0.105773 & 0.046371 & 0.069317 & 0.201588 & 0.075389 & 0.086154 & 0.051239 & 0.195287 \\
\hline $\mathrm{Li}_{2} \mathrm{O}$ & 0.142360 & 0.134655 & 0.136929 & 0.020906 & 0.148721 & 0.148738 & 0.145302 & 0.031392 \\
\hline $\mathrm{CaO}$ & 0.000000 & 0.000000 & 0.000000 & 0.089118 & 0.000000 & 0.090575 & 0.088483 & 0.000000 \\
\hline $\mathrm{MgO}$ & 0.000000 & 0.114094 & 0.116020 & 0.000000 & 0.000000 & 0.000000 & 0.000000 & 0.120123 \\
\hline $\mathrm{Fe}_{2} \mathrm{O}_{3}$ & 0.023891 & 0.007199 & 0.007321 & 0.007824 & 0.007951 & 0.059639 & 0.007768 & 0.007579 \\
\hline $\mathrm{Al}_{2} \mathrm{O}_{3}$ & 0.006236 & 0.038673 & 0.020925 & 0.035414 & 0.059836 & 0.002055 & 0.015634 & 0.046415 \\
\hline $\mathrm{ZOO}_{2}$ & 0.067085 & 0.000000 & 0.000000 & 0.000000 & 0.000000 & 0.000000 & 0.050338 & 0.000000 \\
\hline $\mathrm{Ag}_{0} \mathrm{O}$ & 0.000000 & 0.000000 & 0.000000 & 0.000000 & 0.000000 & 0.000000 & 0.000000 & 0.000000 \\
\hline $\mathrm{As}_{2} \mathrm{O}_{3}$ & 0.000000 & 0.000000 & 0.000000 & 0.000000 & 0.000000 & 0.000000 & 0.000000 & 0.000000 \\
\hline $\mathrm{BaO}$ & 0.000074 & 0.000067 & 0.000682 & 0.000073 & 0.000696 & 0.000420 & 0.000072 & 0.000071 \\
\hline Cdo & 0.000665 & 0.000601 & 0.006113 & 0.000653 & 0.006235 & 0.003765 & 0.000649 & 0.000633 \\
\hline $\mathrm{Ce}_{2} \mathrm{O}_{3}$ & 0.000050 & 0.000045 & 0.000457 & 0.000049 & 0.000466 & 0.000281 & 0.000048 & 0.000047 \\
\hline $\mathrm{CoO}^{-3}$ & 0.000000 & 0.000000 & 0.000000 & 0.000000 & 0.000000 & 0.000000 & 0.000000 & 0.000000 \\
\hline $\mathrm{Cr}_{2} \mathrm{O}_{3}$ & 0.000094 & 0.000085 & 0.000861 & 0.000092 & 0.000879 & 0.000531 & 0.000091 & 0.000089 \\
\hline $\mathrm{Cs}_{2} \mathrm{O}$ & 0.000061 & 0.000055 & 0.000558 & 0.000060 & 0.000569 & 0.000344 & 0.000059 & 0.000058 \\
\hline $\mathrm{CuO}$ & 0.000215 & 0.000194 & 0.001977 & 0.000211 & 0.002016 & 0.001217 & 0.000210 & 0.000205 \\
\hline $\mathbf{F}$ & 0.001797 & 0.001625 & 0.016520 & 0.001766 & 0.016848 & 0.010175 & 0.001753 & 0.001710 \\
\hline $\mathrm{K}_{2} \mathrm{O}$ & 0.000000 & 0.000000 & 0.000000 & 0.000000 & 0.000000 & 0.000000 & 0.000000 & 0.000000 \\
\hline $\mathrm{La}_{2} \mathrm{O}_{3}$ & 0.000218 & 0.000197 & 0.002008 & 0.000215 & 0.002047 & 0.001236 & 0.000213 & 0.000208 \\
\hline $\mathrm{MnO}$ & 0.000197 & 0.000178 & 0.001809 & 0.000193 & 0.001845 & 0.001114 & 0.000192 & 0.000187 \\
\hline $\mathrm{MoO}_{3}$ & 0.000237 & 0.000214 & 0.002181 & 0.000233 & 0.002224 & 0.001343 & 0.000231 & 0.000226 \\
\hline $\mathrm{Nd}_{2} \mathrm{O}_{3}$ & 0.000417 & 0.000377 & 0.003837 & 0.000410 & 0.003914 & 0.002363 & 0.000407 & 0.000397 \\
\hline $\mathrm{NiO}$ & 0.000877 & 0.000793 & 0.008060 & 0.000861 & 0.008220 & 0.004964 & 0.000855 & 0.000834 \\
\hline $\mathrm{P}_{2} \mathrm{O}_{5}$ & 0.000080 & 0.000072 & 0.000737 & 0.000079 & 0.000752 & 0.000454 & 0.000078 & 0.000076 \\
\hline PBO & 0.000000 & 0.000000 & 0.000000 & 0.000000 & 0.000000 & 0.000000 & 0.000000 & 0.000000 \\
\hline Pdo & 0.000047 & 0.000042 & 0.000430 & 0.000046 & 0.000438 & 0.000265 & 0.000046 & 0.000044 \\
\hline $\mathrm{Pr}_{6} \mathrm{O}_{1}$ & 0.000011 & 0.000010 & 0.000102 & 0.000011 & 0.000104 & 0.000063 & 0.000011 & 0.000011 \\
\hline $\mathrm{Rb}_{2} \mathrm{O}$ & 0.000031 & 0.000028 & 0.000281 & 0.000030 & 0.000287 & 0.000173 & 0.000030 & 0.000029 \\
\hline $\mathrm{Rh}_{2} \mathrm{O}_{3}$ & 0.000023 & 0.000020 & 0.000207 & 0.000022 & 0.000211 & 0.000128 & 0.000022 & 0.000021 \\
\hline $\mathrm{RuO}_{2}$ & 0.000129 & 0.000116 & 0.001182 & 0.000126 & 0.001205 & 0.000728 & 0.000125 & 0.000122 \\
\hline $\mathrm{SO}_{3}$ & 0.000392 & 0.000354 & 0.003599 & 0.000385 & 0.003671 & 0.002217 & 0.000382 & 0.000373 \\
\hline $\mathrm{Sb}_{2} \mathrm{O}_{3}$ & 0.000000 & 0.000000 & 0.000000 & 0.000000 & 0.000000 & 0.000000 & 0.000000 & 0.000000 \\
\hline $\mathrm{SeO}_{2}$ & 0.000000 & 0.000000 & 0.000000 & 0.000000 & 0.000000 & 0.000000 & 0.000000 & 0.000000 \\
\hline $\mathrm{Sm}_{2} \mathrm{O}_{3}$ & 0.000016 & 0.000015 & 0.000151 & 0.000016 & 0.000154 & 0.000093 & 0.000016 & 0.000016 \\
\hline $\mathrm{SrO}^{2-3}$ & 0.000110 & 0.000099 & 0.001010 & 0.000108 & 0.001030 & 0.000622 & 0.000107 & 0.000105 \\
\hline $\mathrm{TeO}_{2}$ & 0.000000 & 0.000000 & 0.000000 & 0.000000 & 0.000000 & 0.000000 & 0.000000 & 0.000000 \\
\hline $\mathrm{TiO}_{2}^{-}$ & 0.000000 & 0.000000 & 0.000000 & 0.000000 & 0.000000 & 0.000000 & 0.000000 & 0.000000 \\
\hline $\mathrm{U}_{3} \mathrm{O}_{8}$ & 0.000000 & 0.000000 & 0.000000 & 0.000000 & 0.000000 & 0.000000 & 0.000000 & 0.000000 \\
\hline $\mathrm{WO}_{3}$ & 0.000000 & 0.000000 & 0.000000 & 0.000000 & 0.000000 & 0.000000 & 0.000000 & 0.000000 \\
\hline $\mathrm{Y}_{2} \mathrm{O}_{3}$ & 0.000025 & 0.000023 & 0.000233 & 0.000025 & 0.000238 & 0.000143 & 0.000025 & 0.000024 \\
\hline $\mathrm{ZnO}$ & 0.000000 & 0.000000 & 0.000000 & 0.000000 & 0.000000 & 0.000000 & 0.000000 & 0.000000 \\
\hline PCT B & 0.347 & 3.854 & 9.646 & 0.173 & 4.5 & 4.662 & 1.628 & 3.270 \\
\hline PCT Li & 0.386 & 2.534 & 5.453 & 0.604 & 3.226 & 2.765 & 1.436 & 1.835 \\
\hline PCT Na & 0.279 & 2.089 & 6.097 & 0.809 & 2.060 & 3.526 & 1.349 & 2.342 \\
\hline
\end{tabular}

* The glass names are those used in the reference from which the data were taken. 
Table A.3. CVS-II Phase 2 Glass Compositions (mole fractions) and PCT Normalized $\mathrm{B}, \mathrm{Li}$, and $\mathrm{Na}$ Releases $\left(\mathrm{g} / \mathrm{m}^{2}\right)$ (continued)

\begin{tabular}{|c|c|c|c|c|c|c|c|c|}
\hline Comp. & CVS2-28* & CVS2-29 & CVS2-30 & CVS2-31 & CVS2-32 & CVS2-33 & CVS2-34 & CVS2-35 \\
\hline $\mathrm{SiO}_{2}$ & 0.611440 & 0.495802 & 0.593498 & 0.585354 & 0.575854 & 0.433951 & 0.450027 & 0.448238 \\
\hline & 0.046957 & 0.194901 & 0.197388 & 0.163346 & 0.045638 & 0.048510 & 0.161186 & 0.046054 \\
\hline $\mathrm{Na}_{2} \mathrm{O}$ & 0.210979 & 0.073886 & 0.092234 & 0.049777 & 0.205049 & 0.200325 & 0.207746 & 0.206921 \\
\hline $\mathrm{Li}_{2} \mathrm{O}$ & 0.027569 & 0.022704 & 0.022994 & 0.068349 & 0.091016 & 0.075623 & 0.079502 & 0.091847 \\
\hline $\mathrm{CaO}$ & 0.000000 & 0.096781 & 0.000000 & 0.088021 & 0.000000 & 0.000000 & 0.000000 & 0.091475 \\
\hline MgO & 0.000000 & 0.000000 & 0.000000 & 0.000000 & 0.000000 & 0.123222 & 0.000000 & 0.000000 \\
\hline $\mathrm{Fe}_{2} \mathrm{O}_{3}$ & 0.008188 & 0.008497 & 0.056794 & 0.007728 & 0.007958 & 0.007775 & 0.008063 & 0.025378 \\
\hline $\mathrm{Al}_{2} \mathrm{O}_{3}$ & 0.035589 & 0.000000 & 0.030863 & 0.031831 & 0.001683 & 0.054310 & 0.087640 & 0.084273 \\
\hline $\mathrm{ZrO}_{2}$ & 0.000000 & 0.045919 & 0.000000 & 0.000000 & 0.067041 & 0.000000 & 0.000000 & 0.000000 \\
\hline $\mathrm{Ag}_{2} \mathrm{O}$ & 0.000000 & 0.000000 & 0.000000 & 0.000000 & 0.000000 & 0.000000 & 0.000000 & 0.000000 \\
\hline $\mathrm{As}_{2} \mathrm{O}_{3}$ & 0.000000 & 0.000000 & 0.000000 & 0.000000 & 0.000000 & 0.000000 & 0.000000 & 0.000000 \\
\hline $\mathrm{BaO}$ & 0.000763 & 0.000792 & 0.000080 & 0.000072 & 0.000074 & 0.000725 & 0.000075 & 0.000075 \\
\hline CdO & 0.006838 & 0.007095 & 0.000719 & 0.000645 & 0.000665 & 0.006493 & 0.000673 & 0.000671 \\
\hline $\mathrm{Ce}_{2} \mathrm{O}_{3}$ & 0.000511 & 0.000530 & 0.000054 & 0.000048 & 0.000050 & 0.000485 & 0.000050 & 0.000050 \\
\hline $\mathrm{COO}^{\circ}$ & 0.000000 & 0.000000 & 0.000000 & 0.000000 & 0.000000 & 0.000000 & 0.000000 & 0.000000 \\
\hline $\mathrm{Cr}_{2} \mathrm{O}_{3}$ & 0.000964 & 0.001000 & 0.000101 & 0.000091 & 0.000094 & 0.000915 & 0.000095 & 0.000095 \\
\hline $\mathrm{Cs}_{2} \mathrm{O}$ & 0.000624 & 0.000648 & 0.000066 & 0.000059 & 0.000061 & 0.000593 & 0.000061 & 0.000061 \\
\hline CuO & 0.002211 & 0.002294 & 0.000232 & 0.000209 & 0.000215 & 0.002099 & 0.000218 & 0.000217 \\
\hline $\mathbf{F}$ & 0.018479 & 0.019174 & 0.001942 & 0.001744 & 0.001796 & 0.017546 & 0.001820 & 0.001812 \\
\hline $\mathrm{K}_{2} \mathrm{O}$ & 0.000000 & 0.000000 & 0.000000 & 0.000000 & 0.000000 & 0.000000 & 0.000000 & 0.000000 \\
\hline $\mathrm{La}_{2} \mathrm{O}_{3}$ & 0.002246 & 0.002330 & 0.000236 & 0.000212 & 0.000218 & 0.002132 & 0.000221 & 0.000220 \\
\hline MnO & 0.002023 & 0.002099 & 0.000213 & 0.000191 & 0.000197 & 0.001921 & 0.000199 & 0.000198 \\
\hline $\mathrm{MoO}_{3}$ & 0.002439 & 0.002531 & 0.000256 & 0.000230 & 0.000237 & 0.002316 & 0.000240 & 0.000239 \\
\hline $\mathrm{Nd}_{2} \mathrm{O}_{3}$ & 0.004292 & 0.004454 & 0.000451 & 0.000405 & 0.000417 & 0.004076 & 0.000423 & 0.000421 \\
\hline $\mathrm{NiO}$ & 0.009015 & 0.009355 & 0.000947 & 0.000851 & 0.000876 & 0.008560 & 0.000888 & 0.000884 \\
\hline $\mathrm{P}_{2} \mathrm{O}_{3}$ & 0.000824 & 0.000856 & 0.000087 & 0.000078 & 0.000080 & 0.000783 & 0.000081 & 0.000081 \\
\hline PBO & 0.000000 & 0.000000 & 0.000000 & 0.000000 & 0.000000 & 000 & 0.000000 & 10000 \\
\hline PdO & 0.000481 & 0.000499 & 0.000051 & 0.000045 & 0.000047 & 0.000456 & 0.000047 & 0.000047 \\
\hline $\mathrm{Pr}_{6} \mathrm{O}_{11}$ & 0.000115 & 0.000119 & 0.0000 & 0.000011 & 0.000011 & 0.000109 & 0.000011 & 0.000011 \\
\hline $\mathrm{Rb}_{2} \mathrm{O}$ & 0.000315 & 0.000327 & 0.000033 & 0.000030 & 0.000031 & 0.000299 & 0.000031 & 0.000031 \\
\hline $\mathrm{Rh}_{2} \mathrm{O}_{3}$ & 0.000232 & 0.000241 & 0.000024 & 0.000022 & 0.000023 & 0.000220 & 0.000023 & 0.000023 \\
\hline $\mathrm{RuO}_{2}$ & 0.001322 & 0.001371 & 0.000139 & 0.000125 & 0.000128 & 0.001255 & 0.000130 & 0.000130 \\
\hline $\mathrm{SO}_{3}$ & 0.004026 & 0.004178 & 0.000423 & 0.000380 & 0.000391 & 0.003823 & 0.000396 & 0.000395 \\
\hline $\mathrm{Sb}_{2} \mathrm{O}_{3}$ & 0.000000 & 0.000000 & 0.000000 & 0.000000 & 0.000000 & 0.000000 & 0.000000 & 0.000000 \\
\hline $\mathrm{SeO}_{2}$ & 0.000000 & 0.000000 & 0.000000 & 0.000000 & 0.000000 & 0.000000 & 0.000000 & 0.000000 \\
\hline $\mathrm{Sm}_{2} \mathrm{O}_{3}$ & 0.000169 & 0.000175 & 0.000018 & 0.000016 & 0.000016 & 0.000160 & 0.000017 & 0.000017 \\
\hline Sro & 0.001129 & 0.001172 & 0.000119 & 0.000107 & 0.000110 & 0.001072 & 0.000111 & 0.000111 \\
\hline $\mathrm{TeO}_{2}$ & 0.000000 & 0.000000 & 0.000000 & 0.000000 & 0.000000 & 0.000000 & & 0.000000 \\
\hline $\mathrm{TiO}_{2}$ & 0.000000 & 0.000000 & 0.000000 & 0.000000 & 0.000000 & 0.000000 & 0.000000 & 0.000000 \\
\hline $\mathrm{U}_{3} \mathrm{O}_{2}$ & 0.000000 & 0.000000 & 0.000000 & 0.000000 & 0.000000 & 0.000000 & 0.000000 & 0.000000 \\
\hline $\mathrm{WO}_{3}$ & 0.000000 & 0.000000 & 0.000000 & 0.000000 & 0.000000 & 0.000000 & 0.000000 & 0.000000 \\
\hline $\mathrm{Y}_{2} \mathrm{O}_{3}$ & 0.000261 & 0.000270 & 0.000027 & 0.000025 & 0.000025 & 0.000247 & 0.000026 & 0.000026 \\
\hline $\mathrm{ZnO}$ & 0.000000 & 0.000000 & 0.000000 & 0.000000 & 0.000000 & 0.000000 & 0.000000 & 0.000000 \\
\hline РСТ В & 5.144 & 1.286 & 6.5 & 0.41 & 9.6 & 1.7 & 4.3 & 0.3 \\
\hline PCT Li & & & & 0.460 & & & 3.468 & 0.580 \\
\hline PCT $\mathrm{Na}$ & 3.538 & 1.273 & 2.552 & 0.318 & 5.896 & 1.608 & 2.700 & 0.910 \\
\hline
\end{tabular}

* The glass names are those used in the reference from which the data were taken. 
Table A.3. CVS-II Phase 2 Glass Compositions (mole fractions) and PCT Normalized $\mathrm{B}, \mathrm{Li}$, and $\mathrm{Na}$ Releases $\left(\mathrm{g} / \mathrm{m}^{2}\right)$ (continued)

\begin{tabular}{|c|c|c|c|c|c|c|c|c|}
\hline & CVS2-36* & CVS2-37 & CVS2-38 & CVS2-39 & CVS2-40 & CVS2-41 & CVS2-42 & CVS2-43 \\
\hline $\mathrm{SiO}_{2}$ & 0.561666 & 0.580749 & 0.555715 & 0.482977 & 0.515480 & 0.538664 & 0.576536 & 0.521806 \\
\hline $\mathrm{B}_{2} \mathrm{O}_{3}$ & 0.044710 & 0.073776 & 0.103340 & 0.043079 & 0.056406 & 0.060589 & 0.043840 & 0.131196 \\
\hline $\mathrm{Na}_{2} \mathrm{O}$ & 0.089493 & 0.104795 & 0.109273 & 0.131811 & 0.148726 & 0.076588 & 0.061554 & 0.049887 \\
\hline $\mathrm{Li}_{2} \mathrm{O}$ & 0.145833 & 0.143407 & 0.021710 & 0.140514 & 0.086581 & 0.142382 & 0.142994 & 0.135137 \\
\hline $\mathrm{CaO}$ & 0.088806 & 0.000000 & 0.000000 & 0.000000 & 0.021916 & 0.054190 & 0.034831 & 0.022054 \\
\hline $\mathrm{MgO}$ & 0.000000 & 0.000000 & 0.128764 & 0.119058 & 0.076236 & 0.030160 & 0.057551 & 0.046029 \\
\hline $\mathrm{Fe}_{2} \mathrm{O}_{3}$ & 0.058474 & 0.007667 & 0.058010 & 0.027870 & 0.007696 & 0.011418 & 0.038224 & 0.011617 \\
\hline $\mathrm{Al}_{2} \mathrm{O}_{3}$ & 0.005373 & 0.084056 & 0.017306 & 0.015178 & 0.060271 & 0.059611 & 0.017960 & 0.030325 \\
\hline $\mathrm{ZrO}_{2}$ & 0.000000 & 0.000000 & 0.000000 & 0.034075 & 0.009975 & 0.009865 & 0.009908 & 0.035130 \\
\hline $\mathrm{Ag}_{2} \mathrm{O}$ & 0.000000 & 0.000000 & 0.000000 & 0.000000 & 0.000000 & 0.000000 & 0.000000 & 0.000000 \\
\hline $\mathrm{As}_{2} \mathrm{O}_{3}$ & 0.000000 & 0.000000 & 0.000000 & 0.000000 & 0.000000 & 0.000000 & 0.000000 & 0.000000 \\
\hline $\mathrm{BaO}$ & 0.000073 & 0.000071 & 0.000076 & 0.000070 & 0.000215 & 0.000213 & 0.000214 & 0.000217 \\
\hline CdO & 0.000651 & 0.000640 & 0.000678 & 0.000627 & 0.001928 & 0.001907 & 0.001915 & 0.001940 \\
\hline $\mathrm{Ce}_{2} \mathrm{O}_{3}$ & 0.000049 & 0.000048 & 0.000051 & 0.000047 & 0.000144 & 0.000142 & 0.000143 & 0.000145 \\
\hline $\mathrm{CoO}$ & 0.000000 & 0.000000 & 0.000000 & 0.000000 & 0.000000 & 0.000000 & $0.000000^{\circ}$ & 0.000000 \\
\hline $\mathrm{Cr}_{2} \mathrm{O}_{3}$ & 0.000092 & 0.000090 & 0.000096 & 0.000088 & 0.000272 & 0.000269 & 0.000270 & 0.000273 \\
\hline $\mathrm{Cs}_{2}^{2} \mathrm{O}$ & 0.000059 & 0.000058 & 0.000062 & 0.000057 & 0.000176 & 0.000174 & 0.000175 & 0.000177 \\
\hline $\mathrm{CuO}$ & 0.000211 & 0.000207 & 0.000219 & 0.000203 & 0.000623 & 0.000617 & 0.000619 & 0.000627 \\
\hline $\mathbf{F}$ & 0.001759 & 0.001730 & 0.001833 & 0.001695 & 0.005210 & 0.005153 & 0.005176 & 0.005243 \\
\hline $\mathrm{K}_{2} \mathrm{O}$ & 0.000000 & 0.000000 & 0.000000 & 0.000000 & 0.000000 & 0.000000 & 0.000000 & 0.000000 \\
\hline $\mathrm{La}_{2} \mathrm{O}_{3}$ & 0.000214 & 0.000210 & 0.000223 & 0.000206 & 0.000633 & 0.000626 & 0.000629 & 0.000637 \\
\hline $\mathrm{MnO}$ & 0.000193 & 0.000189 & 0.000201 & 0.000186 & 0.000570 & 0.000564 & 0.000567 & 0.000574 \\
\hline $\mathrm{MoO}_{3}$ & 0.000232 & 0.000228 & 0.000242 & 0.000224 & 0.000688 & 0.000680 & 0.000683 & 0.000692 \\
\hline $\mathrm{Nd}_{2} \mathrm{O}_{3}$ & 0.000409 & 0.000402 & 0.000426 & 0.000394 & 0.001210 & 0.001197 & 0.001202 & 0.001218 \\
\hline $\mathrm{NiO}$ & 0.000858 & 0.000844 & 0.000894 & 0.000827 & 0.002542 & 0.002514 & 0.002525 & 0.002558 \\
\hline $\mathrm{P}_{2} \mathrm{O}_{5}$ & 0.000079 & 0.000077 & 0.000082 & 0.000076 & 0.000232 & 0.000230 & 0.000231 & 0.000234 \\
\hline Pó & 0.000000 & 0.000000 & 0.000000 & 0.000000 & 0.000000 & 0.000000 & 0.000000 & 0.000000 \\
\hline PdO & 0.000046 & 0.000045 & 0.000048 & 0.000044 & 0.000136 & 0.000134 & 0.000135 & 0.000136 \\
\hline $\mathrm{Pr}_{6} \mathrm{O}_{11}$ & 0.000011 & 0.000011 & 0.000011 & 0.000011 & 0.000032 & 0.000032 & 0.000032 & 0.000033 \\
\hline $\mathrm{Rb}_{2} \mathrm{O}$ & 0.000030 & 0.000029 & 0.000031 & 0.000029 & 0.000089 & 0.000088 & 0.000088 & 0.000089 \\
\hline $\mathrm{Rh}_{2} \mathrm{O}_{3}$ & 0.000022 & 0.000022 & 0.000023 & 0.000021 & 0.000065 & 0.000065 & 0.000065 & 0.000066 \\
\hline $\mathrm{RuO}_{2}$ & 0.000126 & 0.000124 & 0.000131 & 0.000121 & 0.000373 & 0.000369 & 0.000370 & 0.000375 \\
\hline $\mathrm{SO}_{3}$ & 0.000383 & 0.000377 & 0.000399 & 0.000369 & 0.001135 & 0.001123 & 0.001128 & 0.001142 \\
\hline $\mathrm{Sb}_{2} \mathrm{O}_{3}$ & 0.000000 & 0.000000 & 0.000000 & 0.000000 & 0.000000 & 0.000000 & 0.000000 & 0.000000 \\
\hline $\mathrm{SeO}_{2}$ & 0.000000 & 0.000000 & 0.000000 & 0.000000 & 0.000000 & 0.000000 & 0.000000 & 0.000000 \\
\hline $\mathrm{Sm}_{2} \mathrm{O}_{3}$ & 0.000016 & 0.000016 & 0.000017 & 0.000015 & 0.000048 & 0.000047 & 0.000047 & 0.000048 \\
\hline StO & 0.000108 & 0.000106 & 0.000112 & 0.000104 & 0.000318 & 0.000315 & 0.000316 & 0.000320 \\
\hline $\mathrm{TeO}_{2}$ & 0.000000 & 0.000000 & 0.000000 & 0.000000 & 0.000000 & 0.000000 & 0.000000 & 0.000000 \\
\hline $\mathrm{TiO}_{2}$ & 0.000000 & 0.000000 & 0.000000 & 0.000000 & 0.000000 & 0.000000 & 0.000000 & 0.000000 \\
\hline $\mathrm{U}_{3} \mathrm{O}_{8}$ & 0.000000 & 0.000000 & 0.000000 & 0.000000 & 0.000000 & 0.000000 & 0.000000 & 0.000000 \\
\hline $\mathrm{WO}_{3}$ & 0.000000 & 0.000000 & 0.000000 & 0.000000 & 0.000000 & 0.000000 & 0.000000 & 0.000000 \\
\hline $\mathrm{Y}_{2} \mathrm{O}_{3}$ & 0.000025 & 0.000024 & 0.000026 & 0.000024 & 0.000073 & 0.000073 & 0.000073 & 0.000074 \\
\hline $\mathrm{ZnO}$ & 0.000000 & 0.000000 & 0.000000 & 0.000000 & 0.000000 & 0.000000 & 0.000000 & 0.000000 \\
\hline РCT B & 0.480 & 0.246 & 1.119 & 12.701 & 0.337 & 0.177 & 1.694 & 0.767 \\
\hline PCT Li & 0.630 & 0.300 & 0.726 & 6.528 & 0.328 & 0.260 & 1.220 & 0.697 \\
\hline $\mathrm{PCT} \mathrm{Na}$ & 0.654 & 0.177 & 0.807 & 7.668 & 0.399 & 0.188 & 1.337 & 0.451 \\
\hline
\end{tabular}

* The glass names are those used in the reference from which the data were taken. 
Table A.3. CVS-II Phase 2 Glass Compositions (mole fractions) and PCT Normalized $\mathrm{B}, \mathrm{Li}$, and $\mathrm{Na}$ Releases $\left(\mathrm{g} / \mathrm{m}^{2}\right)$ (continued)

\begin{tabular}{|c|c|c|c|c|c|c|c|c|}
\hline Comp. & CVS2-44* & CVS2-45 & CVS2-46 & CVS2-47 & CVS2-48 & CVS2-49 & CVS2-50 & CVS2-51 \\
\hline $\mathrm{SiO}_{2}$ & 0.566725 & 0.538631 & 0.537222 & 0.551685 & 0.496072 & 0.537845 & 0.512371 & 0.578911 \\
\hline & 0.092503 & 0.097027 & 0.110893 & 0.044969 & 0.131357 & 0.124169 & 0.105186 & 0.098276 \\
\hline $\mathrm{Na}_{2} \mathrm{O}$ & 0.048193 & 0.049270 & 0.057086 & 0.129007 & 0.099046 & 0.098360 & 0.103769 & 0.118920 \\
\hline $\mathrm{Li}_{2} \mathrm{O}$ & 0.139745 & 0.121611 & 0.143647 & 0.089893 & 0.082770 & 0.088044 & 0.080686 & 0.081492 \\
\hline $\mathrm{CaO}$ & 0.053263 & 0.021781 & 0.021869 & 0.055825 & 0.056542 & 0.022718 & 0.031444 & 0.009546 \\
\hline MgO & 0.029644 & 0.075766 & 0.030428 & 0.031070 & 0.031469 & 0.031610 & 0.057752 & 0.013606 \\
\hline $\mathrm{Fe}_{2} \mathrm{O}_{3}$ & 0.007482 & 0.011779 & 0.007680 & 0.007842 & 0.007942 & 0.020543 & 0.022807 & 0.029966 \\
\hline $\mathrm{Al}_{2} \mathrm{O}_{3}$ & 0.036501 & 0.035460 & 0.044864 & 0.024564 & 0.053116 & 0.049044 & 0.039998 & 0.015047 \\
\hline $\mathrm{ZrO}_{2}$ & 0.009696 & 0.009913 & 0.009953 & 0.025407 & 0.010293 & 0.010340 & 0.022325 & 0.020769 \\
\hline $\mathrm{Ag}_{2} \mathrm{O}$ & 0.000000 & 0.000000 & 0.000000 & 0.000000 & 0.000000 & 0.000000 & 0.000000 & 0.000000 \\
\hline $\mathrm{As}_{2} \mathrm{O}_{3}$ & 0.000000 & 0.000000 & 0.000000 & 0.000000 & 0.000000 & 0.000000 & 0.000000 & 0.000000 \\
\hline $\mathrm{BaO}$ & 0.000209 & 0.000499 & 0.000468 & 0.000512 & 0.000404 & 0.000223 & 0.000305 & 0.000423 \\
\hline CdO & 0.001874 & 0.004471 & 0.004194 & 0.004584 & 0.003621 & 0.001999 & 0.002729 & 0.003316 \\
\hline $\mathrm{Ce}_{2} \mathrm{O}_{3}$ & 0.000140 & 0.000334 & 0.000313 & 0.000342 & 0.000271 & 0.000149 & 0.000204 & 0.000032 \\
\hline $\mathrm{CoO}^{\circ}$ & 0.000000 & 0.000000 & 0.000000 & 0.000000 & 0.000000 & 0.000000 & 0.000000 & 0.000000 \\
\hline $\mathrm{Cr}_{2} \mathrm{O}_{3}$ & 0.000264 & 0.000630 & 0.000591 & 0.000646 & 0.000510 & 0.000282 & 0.000385 & 0.000556 \\
\hline $\mathrm{Cs}_{2} \mathrm{O}$ & 0.000171 & 0.000408 & 0.000383 & 0.000418 & 0.000331 & 0.000182 & 0.000249 & 0.000349 \\
\hline $\mathrm{CuO}^{2}$ & 0.000606 & 0.001446 & 0.001356 & 0.001482 & 0.001171 & 0.000646 & 0.000883 & 0.001301 \\
\hline $\mathbf{F}$ & 0.005065 & 0.012083 & 0.011334 & 0.012387 & 0.009786 & 0.005401 & 0.007376 & 0.006551 \\
\hline $\mathrm{K}_{2} \mathrm{O}$ & 0.000000 & 0.000000 & 0.000000 & 0.000000 & 0.000000 & 0.000000 & 0.000000 & 0.000000 \\
\hline $\mathrm{La}_{2} \mathrm{O}_{3}$ & 0.000616 & 0.001468 & 0.001377 & 0.001505 & 0.001189 & 0.000656 & 0.000896 & 0.003097 \\
\hline Mno & 0.000555 & 0.001323 & 0.001241 & 0.001356 & 0.001071 & 0.000591 & 0.000808 & 0.001191 \\
\hline $\mathrm{MoO}_{3}$ & 0.000669 & 0.001595 & 0.001496 & 0.001635 & 0.001292 & 0.000713 & 0.000974 & 0.001411 \\
\hline $\mathrm{Nd}_{2} \mathrm{O}_{3}$ & 0.001177 & 0.002807 & 0.002633 & 0.002877 & 0.002273 & 0.001255 & 0.001713 & 0.001110 \\
\hline $\mathrm{NiO}^{\circ}$ & 0.002471 & 0.005895 & 0.005530 & 0.006043 & 0.004774 & 0.002635 & 0.003599 & 0.005262 \\
\hline $\mathrm{P}_{2} \mathrm{O}_{5}$ & 0.000226 & 0.000539 & 0.000506 & 0.000553 & 0.000437 & 0.000241 & 0.000329 & 0.000457 \\
\hline POO & 0.000000 & 0.000000 & 0.000000 & 0.000000 & 0.000000 & 0.000000 & 0.000000 & 0.000000 \\
\hline PdO & 0.000132 & 0.000314 & 0.000295 & 0.000322 & 0.000255 & 0.000141 & 0.000192 & 0.000000 \\
\hline $\mathrm{Pr}_{8} \mathrm{O}_{11}$ & 0.000031 & 0.000075 & 0.000070 & 0.000077 & 0.000061 & 0.000033 & 0.000046 & 0.000121 \\
\hline $\mathrm{Rb}_{2} \mathrm{O}$ & 0.000086 & 0.000206 & 0.000193 & 0.000211 & 0.000167 & 0.000092 & 0.000126 & 0.000000 \\
\hline $\mathrm{Rh}_{2} \mathrm{O}_{3}$ & 0.000064 & 0.000152 & 0.000142 & 0.000155 & 0.000123 & 0.000068 & 0.000093 & 0.000000 \\
\hline $\mathrm{RuO}_{2}$ & 0.000362 & 0.000864 & 0.000811 & 0.000886 & .0 .000700 & 0.000386 & 0.000528 & 0.000743 \\
\hline $\mathrm{SO}_{3}{ }^{2}$ & 0.001104 & 0.002633 & 0.002470 & 0.002699 & 0.002132 & 0.001177 & 0.001607 & 0.003601 \\
\hline $\mathrm{Sb}_{2} \mathrm{O}_{3}$ & 0.000000 & 0.000000 & 0.000000 & 0.000000 & 0.000000 & 0.000000 & 0.000000 & 0.000000 \\
\hline & 0.000000 & 0.000000 & 0.000000 & 0.000000 & 0.000000 & 0.000000 & 0.000000 & 0.000000 \\
\hline $\mathrm{Sm}_{2} \mathrm{O}_{3}$ & 0.000046 & 0.000110 & 0.000103 & 0.000113 & 0.000089 & 0.000049 & 0.000067 & 0.000000 \\
\hline $\mathrm{SrO}^{\circ}$ & 0.000310 & 0.000739 & 0.000693 & 0.000757 & 0.000598 & 0.000330 & 0.000451 & 0.000000 \\
\hline $\mathrm{TeO}_{2}$ & 0.000000 & 0.000000 & 0.000000 & 0.000000 & 0.000000 & 0.000000 & 0.000000 & 0.000000 \\
\hline $\mathrm{TiO}_{2}^{2}$ & 0.000000 & 0.000000 & 0.000000 & 0.000000 & 0.000000 & 0.000000 & 0.000000 & 0.000000 \\
\hline $\mathrm{U}_{3} \mathrm{O}_{8}$ & 0.000000 & 0.000000 & 0.000000 & 0.000000 & 0.000000 & 0.000000 & 0.000000 & 0.000000 \\
\hline $\mathrm{wo}_{3}$ & 0.000000 & 0.000000 & 0.000000 & 0.000000 & 0.000000 & 0.000000 & 0.000000 & 0.000000 \\
\hline $\mathrm{Y}_{2} \mathrm{O}_{3}$ & 0.000071 & 0.000170 & 0.000160 & 0.000175 & 0.000138 & 0.000076 & 0.000104 & 0.000000 \\
\hline $\mathrm{ZnO}$ & 0.000000 & 0.000000 & 0.000000 & 0.000000 & 0.000000 & 0.000000 & 0.000000 & 0.003945 \\
\hline РСТ В & 0.255 & 0.500 & 0.317 & 1.15 & 0.3 & 0.3 & 0.4 & 1.764 \\
\hline PCT Li & & & & 1.124 & & & 0.426 & 1.496 \\
\hline PCT $\mathrm{Na}$ & 0.194 & 0.374 & 0.188 & 1.055 & 0.284 & 0.217 & 0.368 & 1.283 \\
\hline
\end{tabular}

* The glass names are those used in the reference from which the data were taken. 
Table A.3. CVS-II Phase 2 Glass Compositions (mole fractions) and PCT Normalized $\mathrm{B}, \mathrm{Li}$, and $\mathrm{Na}$ Releases $\left(\mathrm{g} / \mathrm{m}^{2}\right)$ (continued)

\begin{tabular}{|c|c|c|c|c|c|c|c|}
\hline Comp. & CVS2-52* & CVS2-53 & CVS2-54 & CVS2-55 & CVS2-56 & CVS2-57 & CVS2-58 \\
\hline $\mathrm{SiO}_{2}$ & 0.620786 & 0.538830 & 0.578728 & 0.574707 & 0.569131 & 0.585337 & 0.390482 \\
\hline $\mathrm{B}_{2} \mathrm{O}_{3}$ & 0.072955 & 0.077774 & 0.098245 & 0.097563 & 0.096616 & 0.099367 & 0.172825 \\
\hline $\mathrm{Na}_{2} \mathrm{O}$ & 0.045135 & 0.069967 & 0.118882 & 0.118056 & 0.116911 & 0.120240 & 0.048531 \\
\hline $\mathrm{Li}_{2} \mathrm{O}$ & 0.163937 & 0.124392 & 0.081466 & 0.080900 & 0.080116 & 0.082397 & 0.140927 \\
\hline $\mathrm{CaO}$ & 0.000887 & 0.000000 & 0.009543 & 0.009477 & 0.009385 & 0.009652 & 0.021455 \\
\hline MgO & 0.001388 & 0.076853 & 0.013602 & 0.013507 & 0.013376 & 0.013757 & 0.119408 \\
\hline $\mathrm{Fe}_{2} \mathrm{O}_{3}$ & 0.028028 & 0.015517 & 0.029956 & 0.029748 & 0.029460 & 0.030298 & 0.007534 \\
\hline $\mathrm{Al}_{2} \mathrm{O}_{3}$ & 0.014206 & 0.048607 & 0.015042 & 0.014937 & 0.014792 & 0.015214 & 0.088502 \\
\hline $\mathrm{ZrO}_{2}$ & 0.019424 & 0.005028 & 0.020762 & 0.020618 & 0.020418 & 0.021000 & 0.004882 \\
\hline $\mathrm{Ag}_{2} \mathrm{O}$ & 0.000000 & 0.000000 & 0.000000 & 0.000000 & 0.000000 & 0.000000 & 0.000000 \\
\hline $\mathrm{As}_{2} \mathrm{O}_{3}$ & 0.000000 & 0.000000 & 0.000000 & 0.000000 & 0.000000 & 0.000000 & 0.000000 \\
\hline $\mathrm{BaO}$ & 0.000428 & 0.000579 & 0.000454 & 0.000000 & 0.000000 & 0.000000 & 0.000070 \\
\hline $\mathrm{CdO}$ & 0.003836 & 0.005183 & 0.004068 & 0.000000 & 0.000000 & 0.000000 & $0.0,00629$ \\
\hline $\mathrm{Ce}_{2} \mathrm{O}_{3}$ & 0.000287 & 0.000387 & 0.000304 & 0.000000 & 0.000000 & 0.000000 & $0: 000047$ \\
\hline $\mathrm{CoO}$ & 0.000000 & 0.000000 & 0.000000 & 0.000000 & 0.000000 & 0.000000 & 0.000000 \\
\hline $\mathrm{Cr}_{2} \mathrm{O}_{3}$ & 0.000541 & 0.000730 & 0.000573 & 0.003812 & 0.003020 & 0.002330 & 0.000089 \\
\hline $\mathrm{Cs}_{2} \mathrm{O}$ & 0.000350 & 0.000473 & 0.000371 & 0.000000 & 0.000000 & 0.000000 & 0.000057 \\
\hline $\mathrm{CuO}$ & 0.001240 & 0.001676 & 0.001315 & 0.000000 & 0.000000 & 0.000000 & 0.000203 \\
\hline $\mathbf{F}$ & 0.010366 & 0.014007 & 0.010993 & 0.010165 & 0.010066 & 0.010353 & 0.001700 \\
\hline $\mathbf{K}_{2} \mathbf{O}$ & 0.000000 & 0.000000 & 0.000000 & 0.000000 & 0.000000 & 0.000000 & 0.000000 \\
\hline $\mathrm{La}_{2} \mathrm{O}_{3}$ & 0.001260 & 0.001702 & 0.001336 & 0.000000 & 0.000000 & 0.000000 & 0.000207 \\
\hline $\mathrm{MnO}$ & 0.001135 & 0.001533 & 0.001204 & 0.020882 & 0.034320 & 0.000000 & 0.000186 \\
\hline $\mathrm{MoO}_{3}$ & 0.001368 & 0.001849 & 0.001451 & 0.000000 & 0.000000 & 0.000000 & 0.000224 \\
\hline $\mathrm{Nd}_{2} \mathrm{O}_{3}$ & 0.002408 & 0.000000 & 0.000000 & 0.003214 & 0.000000 & 0.010055 & 0.000395 \\
\hline $\mathrm{NiO}$ & 0.005057 & 0.006834 & 0.005363 & 0.000000 & 0.000000 & 0.000000 & 0.000829 \\
\hline $\mathrm{P}_{2} \mathrm{O}_{5}$ & 0.000463 & 0.000625 & 0.000491 & 0.000000 & 0.000000 & 0.000000 & 0.000076 \\
\hline $\mathrm{PbO}$ & 0.000000 & 0.000000 & 0.000000 & 0.000000 & 0.000000 & 0.000000 & 0.000000 \\
\hline PdO & 0.000270 & 0.000364 & 0.000286 & 0.000000 & 0.000000 & 0.000000 & 0.000044 \\
\hline $\mathrm{Pr}_{6} \mathrm{O}_{11}$ & 0.000064 & 0.000087 & 0.000068 & 0.000000 & 0.000000 & 0.000000 & 0.000011 \\
\hline $\mathrm{Rb}_{2} \mathrm{O}$ & 0.000177 & 0.000239 & 0.000187 & 0.000000 & 0.000000 & 0.000000 & 0.000029 \\
\hline $\mathrm{Rh}_{2} \mathrm{O}_{3}$ & 0.000130 & 0.000176 & 0.000138 & 0.000000 & 0.000000 & 0.000000 & 0.000021 \\
\hline $\mathrm{RuO}_{2}$ & 0.000741 & 0.001002 & 0.000786 & 0.000000 & 0.000000 & 0.000000 & 0.000122 \\
\hline $\mathrm{SO}_{3}$ & 0.002259 & 0.003052 & 0.002395 & 0.002412 & 0.002389 & 0.000000 & 0.000370 \\
\hline $\mathrm{Sb}_{2} \mathrm{O}_{3}$ & 0.000000 & 0.000000 & 0.000000 & 0.000000 & 0.000000 & 0.000000 & 0.000000 \\
\hline $\mathrm{SeO}_{2}$ & 0.000000 & 0.000000 & 0.000000 & 0.000000 & 0.000000 & 0.000000 & 0.000000 \\
\hline $\mathrm{Sm}_{2} \mathrm{O}_{3}$ & 0.000095 & 0.000128 & 0.000100 & 0.000000 & 0.000000 & 0.000000 & 0.000016 \\
\hline Sro & 0.000634 & 0.000856 & 0.000672 & 0.000000 & 0.000000 & 0.000000 & 0.000104 \\
\hline $\mathrm{TeO}_{2}$ & 0.000000 & 0.000000 & 0.000000 & 0.000000 & 0.000000 & 0.000000 & 0.000000 \\
\hline $\mathrm{TiO}_{2}$ & 0.000000 & 0.000000 & 0.000000 & 0.000000 & 0.000000 & 0.000000 & 0.000000 \\
\hline $\mathrm{U}_{3} \mathrm{O}_{8}$ & 0.000000 & 0.001351 & 0.001061 & 0.000000 & 0.000000 & 0.000000 & 0.000000 \\
\hline $\mathrm{WO}_{3}$ & 0.000000 & 0.000000 & 0.000000 & 0.000000 & 0.000000 & 0.000000 & 0.000000 \\
\hline $\mathrm{Y}_{2} \mathrm{O}_{3}$ & 0.000146 & 0.000198 & 0.000155 & 0.000000 & 0.000000 & 0.000000 & 0.000024 \\
\hline $\mathrm{ZnO}$ & 0.000000 & 0.000000 & 0.000000 & 0.000000 & 0.000000 & 0.000000 & 0.000000 \\
\hline РCT B & 0.557 & 0.304 & 2.761 & 1.342 & 1.419 & 1.164 & 0.778 \\
\hline PCT Li & 0.554 & 0.305 & 1.715 & 1.127 & 1.218 & 1.081 & 0.636 \\
\hline PCT $\mathrm{Na}$ & 0.260 & 0.202 & 1.612 & 0.880 & 0.946 & 0.842 & 0.620 \\
\hline
\end{tabular}

* The glass names are those used in the reference from which the data were taken. 
Table A.4. CVS-II Phase 3 Glass Compositions (mole fractions) and PCT Normalized B, Li, and Na Releases $\left(\mathrm{g} / \mathrm{m}^{2}\right)$

\begin{tabular}{|c|c|c|c|c|c|c|c|c|}
\hline Comp. & CVS2-59* & CVS2-60 & CVS2-61 & CVS2-62 & CVS2-63 & CVS2-64 & CVS2-65 & CVS2-66 \\
\hline & 0.446571 & 0.528447 & 0.546037 & 0.586772 & 0.362325 & 0.609524 & 0.560856 & 0.545773 \\
\hline & 0.151175 & 0.075654 & 0.059254 & 0.160213 & 0.166126 & 0.047001 & 0.102172 & 0.083466 \\
\hline & 0.125328 & 0.167335 & 0.120282 & 0.114704 & 0.206488 & 0.095940 & 0.087498 & 0.123811 \\
\hline $\mathrm{Li}_{2} \mathrm{O}$ & 0.149043 & 0.149497 & 0.151771 & 0.032991 & 0.011496 & 0.138115 & .0 .147088 & 0.110908 \\
\hline $\mathrm{CaO}$ & 0.040964 & 0.006754 & 0.000000 & 0.056343 & 0.120111 & 0.002866 & 0.000787 & 0.010960 \\
\hline $\mathrm{MgO}$ & 0.000760 & 0.000746 & 0.0000000 & 0.000784 & 0.000000 & 0.001276 & 0.000626 & 0.009590 \\
\hline $\mathrm{Fe}_{2} \mathrm{O}_{3}$ & 0.007672 & 0.007530 & 0.007781 & 0.007914 & 0.008436 & 0.032687 & 0.000513 & 0.015396 \\
\hline $\mathrm{Al}_{2} \mathrm{O}_{3}$ & 0.069094 & 0.054545 & 0.099011 & 0.030989 & 0.118912 & 0.018158 & 0.012122 & 0.073335 \\
\hline $\mathrm{ZrO}_{2}$ & 0.003729 & 0.003660 & 0.008823 & 0.003846 & 0.000000 & 0.022486 & 0.079220 & 0.001337 \\
\hline $\mathrm{Ag}_{2} \mathrm{O}$ & 0.000000 & 0.000000 & 0.000000 & 0.000000 & 0.000000 & 0.000000 & 0.000000 & 0.000000 \\
\hline $\mathrm{As}_{2} \mathrm{O}_{3}$ & 0.000000 & 0.000000 & 0.000000 & 0.000000 & 0.000000 & 0.000000 & 0.000000 & 0.000000 \\
\hline $\mathrm{BaO}$ & 0.000073 & 0.000075 & 0.000091 & 0.000070 & 0.000079 & 0.000000 & 0.000000 & 0.000209 \\
\hline Cdo & 0.000654 & 0.000573 & 0.000812 & $0.000628^{\circ}$ & 0.000704 & 0.006476 & 0.000000 & 0.000000 \\
\hline $\mathrm{Ce}_{2} \mathrm{O}_{3}$ & 0.000049 & 0.000050 & 0.000061 & 0.000047 & 0.000053 & 0.000483 & 0.000000 & 0.000000 \\
\hline & 0.000000 & 0.000000 & 0.000000 & 0.000000 & 0.000000 & 0.000000 & 0.000000 & 0.000000 \\
\hline $\mathrm{Cr}_{2} \mathrm{O}_{3}$ & 0.000092 & 0.000095 & 0.000114 & 0.000088 & 0.000099 & 0.000477 & 0.001996 & 0.004486 \\
\hline $\mathrm{Cs}_{2} \mathrm{O}$ & 0.000060 & 0.000061 & 0.000074 & 0.000057 & 0.000064 & 0.000590 & 0.000000 & 0.000000 \\
\hline CuO & 0.000211 & 0.000218 & 0.000263 & 0.000203 & 0.000228 & 0.000000 & 0.000000 & 0.000000 \\
\hline $\mathbf{F}$ & 0.001766 & 0.001818 & 0.002195 & 0.001697 & 0.001904 & 0.001122 & 0.000000 & 0.002111 \\
\hline $\mathrm{K}_{2} \mathrm{O}$ & 0.000000 & 0.000000 & 0.000000 & 0.000000 & 0.000000 & 0.000588 & 0.005844 & 0.000000 \\
\hline $\mathrm{La}_{2} \mathrm{O}_{3}$ & 0.000215 & 0.000221 & 0.000267 & 0.000206 & 0.000231 & 0.000549 & 0.000083 & 0.000829 \\
\hline $\mathrm{MnO}^{\circ}$ & 0.000193 & 0.000199 & 0.000240 & 0.000186 & 0.000208 & 0.000000 & 0.000000 & 0.010581 \\
\hline $\mathrm{MoO}_{3}$ & 0.000233 & 0.000240 & $0.000290^{\circ}$ & 0.000224 & 0.000251 & 0.001066 & 0.000000 & 0.002768 \\
\hline $\mathrm{Nd}_{2} \mathrm{O}_{3}$ & 0.000410 & 0.000422 & 0.000510 & 0.000394 & 0.000442 & 0.002788 & 0.001160 & 0.000000 \\
\hline $\mathrm{NiO}^{\circ}$ & 0.000862 & 0.000887 & 0.001071 & 0.000828 & 0.000929 & 0.008450 & 0.000000 & 0.004439 \\
\hline $\mathrm{P}_{2} \mathrm{O}_{3}$ & 0.000079 & 0.000081 & 0.000098 & 0.000076 & 0.000085 & 0.001681 & 0.000000 & 0.000000 \\
\hline PbO & 0.000000 & 0.000000 & 0.000000 & 0.000000 & 0.000000 & 0.000802 & 0.000000 & 0.000000 \\
\hline PdO & 0.000046 & 0.000047 & 0.000057 & 0.000044 & 0.000050 & 0.000279 & 0.000000 & 0.000000 \\
\hline $\mathrm{Pr}_{6} \mathrm{O}_{11}$ & 0.000011 & 0.000011 & 0.000014 & 0.000011 & 0.000012 & 0.000000 & 0.000000 & 0.000000 \\
\hline $\mathrm{Rb}_{2} \mathrm{O}$ & 0.000030 & 0.000031 & 0.000037 & 0.000029 & 0.000032 & 0.000000 & 0.000000 & 0.000000 \\
\hline $\mathrm{Rh}_{2} \mathrm{O}_{3}$ & 0.000022 & 0.000023 & 0.000028 & 0.000021 & 0.000024 & 0.000118 & 0.000035 & 0.000000 \\
\hline $\mathrm{RuO}_{2}$ & 0.000126 & 0.000130 & 0.000157 & 0.000121 & 0.000136 & 0.000852 & 0.000000 & 0.000000 \\
\hline $\mathrm{So}_{3}$ & 0.000385 & 0.000396 & 0.000478 & 0.000370 & 0.000415 & 0.002236 & 0.000000 & 0.000000 \\
\hline $\mathrm{Sb}_{2} \mathrm{O}_{3}$ & 0.000000 & 0.000000 & 0.000000 & 0.000000 & 0.000000 & 0.000000 & 0.000000 & 0.000000 \\
\hline & 0.000000 & 0.000000 & 0.000000 & 0.000000 & 0.000000 & 0.000000 & 0.000000 & 0.000000 \\
\hline & 0.000016 & 0.000017 & 0.000020 & 0.000015 & 0.000017 & 0.000000 & 0.000000 & 0.000000 \\
\hline Sró & 0.000108 & 0.000111 & 0.000134 & 0.000104 & 0.000116 & 0.000000 & 0.000000 & 0.000000 \\
\hline $\mathrm{reO}_{2}$ & 0.000000 & 0.000000 & 0.000000 & 0.000000 & 0.000000 & 0.000000 & 0.000000 & 0.000000 \\
\hline $\mathrm{TiO}_{2}$ & 0.000000 & 0.000000 & 0.000000 & 0.000000 & 0.000000 & 0.002240 & 0.000000 & 0.000000 \\
\hline $\mathrm{U}_{3} \mathrm{O}_{8}$ & 0.000000 & 0.000000 & 0.000000 & 0.000000 & 0.000000 & 0.000000 & 0.000000 & 0.000000 \\
\hline $\mathrm{WO}_{3}$ & 0.000000 & 0.000000 & 0.000000 & 0.000000 & 0.000000 & 0.000000 & 0.000000 & 0.000000 \\
\hline $\mathrm{Y}_{2} \mathrm{O}_{3}$ & 0.000025 & 0.000026 & 0.000031 & 0.000024 & 0.000027 & 0.000000 & 0.000000 & 0.000000 \\
\hline $\mathrm{Zno}$ & 0.000000 & 0.000000 & 0.000000 & 0.000000 & 0.000000 & 0.001152 & 0.000000 & 0.000000 \\
\hline PCT B & 1.591 & 1.624 & 0.222 & 1.002 & 0.332 & 0.379 & 0.335 & 0.210 \\
\hline PCT Li & 1.287 & 1.158 & 0.280 & 0.862 & 0.222 & 0.376 & 0.355 & 0.247 \\
\hline PCT Na & 1.222 & 1.523 & 0.230 & 0.928 & 0.390 & 0.342 & 0.200 & 0.223 \\
\hline
\end{tabular}

* The glass names are those used in the reference from which the data were taken. 
Table A.4. CVS-II Phase 3 Glass Compositions (mole fractions) and PCT Normalized B, Li, and Na Releases $\left(\mathrm{g} / \mathrm{m}^{2}\right)$ (continued)

\begin{tabular}{|c|c|c|c|c|c|c|c|c|}
\hline Comp. & CVS2-67* & CVS2-68 & CVS2-69 & CVS2-70 & CVS2-71 & CVS2-72 & CVS2-73 & CVS2-74 \\
\hline $\mathrm{SiO}_{2}$ & 0.484279 & 0.518857 & 0.587706 & 0.509864 & 0.605472 & 0.546417 & 0.524842 & 0.586424 \\
\hline $\mathrm{B}_{2} \mathrm{O}_{3}$ & 0.144322 & 0.120392 & 0.069990 & 0.128550 & 0.046688 & 0.083565 & 0.121780 & 0.069837 \\
\hline $\mathrm{Na}_{2} \mathrm{O}$ & 0.110933 & 0.079541 & 0.066838 & 0.082685 & 0.095302 & 0.123957 & 0.080458 & 0.066693 \\
\hline $\mathrm{Li}_{2} \mathrm{O}$ & 0.123522 & 0.144074 & 0.148865 & 0.145946 & 0.137196 & 0.111039 & 0.145736 & 0.148541 \\
\hline $\mathrm{CaO}$ & 0.002709 & 0.000772 & 0.008789 & 0.000900 & 0.002847 & 0.010973 & 0.000781 & 0.008770 \\
\hline $\mathrm{MgO}$ & 0.000157 & 0.000307 & 0.004953 & 0.001253 & 0.001267 & 0.009602 & 0.000310 & 0.004943 \\
\hline $\mathrm{Fe}_{2} \mathrm{O}_{3}$ & 0.000159 & 0.001782 & 0.013049 & 0.003162 & 0.032470 & 0.015414 & 0.001802 & 0.013020 \\
\hline $\mathrm{Al}_{2} \mathrm{O}_{3}$ & 0.126855 & 0.099491 & 0.049930 & 0.112593 & 0.018037 & 0.073421 & 0.100639 & 0.049821 \\
\hline $\mathrm{ZrO}_{2}$ & 0.000000 & 0.000050 & 0.000253 & 0.000256 & 0.022336 & 0.001339 & 0.000051 & 0.000253 \\
\hline $\mathrm{Ag}_{2} \mathrm{O}$ & 0.000000 & 0.000000 & 0.000000 & 0.000000 & 0.000000 & 0.000000 & 0.000000 & 0.000000 \\
\hline $\mathrm{As}_{2} \mathrm{O}_{3}$ & 0.000000 & 0.000000 & 0.000000 & 0.000340 & 0.000000 & 0.000000 & 0.000000 & 0.000000 \\
\hline $\mathrm{BaO}$ & 0.000000 & 0.000000 & 0.000000 & 0.000000 & 0.000494 & 0.000313 & 0.000304 & 0.000666 \\
\hline CdO & 0.000000 & 0.000000 & 0.000534 & 0.000538 & 0.004428 & 0.002800 & 0.002722 & 0.005964 \\
\hline $\mathrm{Ce}_{2} \mathrm{O}_{3}$ & 0.000000 & 0.000000 & 0.000000 & 0.000240 & 0.000331 & 0.000209 & 0.000203 & 0.000446 \\
\hline $\mathrm{COO}^{-}$ & 0.000000 & 0.000000 & 0.000000 & 0.000000 & 0.000000 & 0.000000 & 0.000000 & 0.000000 \\
\hline $\mathrm{Cr}_{2} \mathrm{O}_{3}$ & 0.000563 & 0.012071 & 0.009750 & 0.004852 & 0.000624 & 0.000395 & 0.000384 & 0.000840 \\
\hline $\mathrm{Cs}_{2} \mathrm{O}$ & 0.000000 & 0.000000 & 0.000000 & 0.000000 & 0.000404 & 0.000256 & 0.000248 & 0.000544 \\
\hline $\mathrm{CuO}$ & 0.000000 & 0.000000 & 0.001437 & 0.001304 & 0.001432 & 0.000905 & 0.000880 & 0.001928 \\
\hline $\mathbf{F}$ & 0.004280 & 0.018418 & 0.003609 & 0.000000 & 0.011966 & 0.007567 & 0.007356 & 0.016116 \\
\hline $\mathrm{K}_{2} \mathrm{O}$ & 0.000000 & 0.001065 & $0.000000^{\circ}$ & 0.000000 & 0.000000 & 0.000000 & 0.000000 & 0.000000 \\
\hline $\mathrm{La}_{2} \mathrm{O}_{3}$ & 0.000000 & 0.000000 & 0.000000 & 0.000106 & 0.001454 & 0.000919 & 0.000894 & 0.001958 \\
\hline Mno & 0.000000 & 0.001894 & 0.010728 & 0.002562 & 0.001310 & 0.000828 & 0.000805 & 0.001764 \\
\hline $\mathrm{MoO}_{3}$ & 0.000000 & 0.000304 & 0.000000 & 0.000000 & 0.001579 & 0.000999 & 0.000971 & 0.002127 \\
\hline $\mathrm{Nd}_{2} \mathrm{O}_{3}$ & 0.000000 & 0.000000 & 0.000323 & 0.000103 & 0.002779 & 0.001758 & 0.001709 & 0.003744 \\
\hline $\mathrm{NiO}$ & 0.000000 & 0.000000 & 0.000000 & 0.000693 & 0.005838 & 0.003692 & 0.003589 & 0.007863 \\
\hline $\mathrm{P}_{2} \mathrm{O}_{5}$ & 0.002203 & 0.000000 & 0.014309 & 0.000297 & 0.000534 & 0.000338 & 0.000328 & 0.000719 \\
\hline $\mathrm{PbO}$ & 0.000000 & 0.000000 & 0.000410 & 0.000000 & 0.000000 & 0.000000 & 0.000000 & 0.000000 \\
\hline PdO & 0.000000 & 0.000000 & 0.000700 & 0.000706 & 0.000311 & 0.000197 & 0.000191 & 0.000419 \\
\hline $\mathrm{Pr}_{6} \mathrm{O}_{11}$ & 0.000000 & 0.000000 & 0.000000 & 0.000000 & 0.000074 & 0.000047 & 0.000046 & 0.000100 \\
\hline $\mathrm{Rb}_{2} \mathrm{O}$ & 0.000000 & 0.000000 & 0.000000 & 0.000000 & 0.000204 & 0.000129 & 0.000125 & 0.000275 \\
\hline $\mathrm{Rh}_{2} \mathrm{O}_{3}$ & 0.00000 & 0.000000 & 0.000158 & 0.000166 & 0.000150 & 0.000095 & 0.000092 & 0.000202 \\
\hline $\mathrm{RuO}_{2}$ & 0.000000 & 0.000000 & 0.000301 & 0.000337 & 0.000856 & 0.000541 & 0.000526 & 0.001153 \\
\hline $\mathrm{SO}_{3}$ & 0.000018 & 0.000064 & 0.005361 & 0.000216 & 0.002607 & 0.001649 & 0.001603 & 0.003512 \\
\hline $\mathrm{Sb}_{2} \mathrm{O}_{3}$ & 0.000000 & 0.000000 & 0.000000 & 0.000106 & 0.000000 & 0.000000 & 0.000000 & 0.000000 \\
\hline $\mathrm{SeO}_{2}$ & 0.000000 & 0.000000 & 0.002009 & 0.002007 & 0.000000 & 0.000000 & 0.000000 & 0.000000 \\
\hline $\mathrm{Sm}_{2} \mathrm{O}_{3}$ & 0.000000 & 0.000000 & 0.000000 & 0.000000 & 0.000109 & 0.000069 & 0.000067 & 0.000147 \\
\hline Sro & 0.000000 & 0.000000 & 0.000000 & 0.000000 & 0.000731 & 0.000462 & 0.000450 & 0.000985 \\
\hline $\mathrm{TeO}_{2}$ & 0.000000 & 0.000000 & 0.000000 & 0.000217 & 0.000000 & 0.000000 & 0.000000 & 0.000000 \\
\hline $\mathrm{TiO}_{2}^{-}$ & 0.000000 & 0.000000 & 0.000000 & 0.000000 & 0.000000 & 0.000000 & 0.000000 & 0.000000 \\
\hline $\mathrm{U}_{3} \mathrm{O}_{8}$ & 0.000000 & 0.000000 & 0.000000 & 0.000000 & 0.000000 & 0.000000 & 0.000000 & 0.000000 \\
\hline $\mathrm{WO}_{3}$ & 0.000000 & 0.000000 & 0.000000 & 0.000000 & 0.000000 & 0.000000 & 0.000000 & 0.000000 \\
\hline $\mathrm{Y}_{2} \mathrm{O}_{3}$ & 0.000000 & 0.000000 & 0.000000 & 0.000000 & 0.000169 & 0.000107 & 0.000104 & 0.000227 \\
\hline $\mathrm{ZnO}$ & 0.000000 & 0.000917 & 0.000000 & 0.000000 & 0.000000 & 0.000000 & 0.000000 & 0.000000 \\
\hline РСT B & 0.512 & 0.308 & 0.226 & 0.312 & 0.411 & 0.210 & 0.244 & 0.226 \\
\hline PCT Li & & 0.340 & 0.302 & 0.381 & 0.427 & 0.226 & 0.301 & 0.279 \\
\hline PCT Na & 0.214 & 0.127 & 0.112 & 0.128 & 0.350 & 0.228 & 0.074 & 0.147 \\
\hline
\end{tabular}

* The glass names are those used in the reference from which the data were taken. 
Table A.4. CVS-II Phase 3 Glass Compositions (mole fractions) and PCT Normalized $\mathrm{B}, \mathrm{Li}$, and $\mathrm{Na}$ Releases $\left(\mathrm{g} / \mathrm{m}^{2}\right)$ (continued)

\begin{tabular}{|c|c|c|c|c|c|c|c|c|}
\hline Comp. & CVS2-75* & CVS2-76 & CVS2-77 & CVS2-78 & CVS2-79 & CVS2-80 & CVS2-81 & CVS2-82 \\
\hline $\mathrm{SiO}_{2}$ & 0.508511 & 0.517269 & 0.478355 & 0.598395 & 0.626631 & 0.568136 & 0.456525 & 0.533726 \\
\hline & 0.128208 & 0.053380 & 0.054442 & 0.147557 & 0.157932 & 0.154619 & 0.053728 & 0.078144 \\
\hline & 0.082465 & 0.179876 & 0.183453 & 0.051795 & 0.055436 & 0.054273 & 0.174411 & 0.166309 \\
\hline $\mathrm{Li}_{2} \mathrm{O}$ & 0.145559 & 0.130997 & 0.147978 & 0.054575 & 0.027826 & 0.039626 & 0.146039 & 0.134149 \\
\hline $\mathrm{CaO}$ & 0.000898 & 0.044177 & 0.005632 & 0.005724 & 0.006127 & 0.005998 & 0.005558 & 0.005106 \\
\hline $\mathrm{MgO}$ & 0.001249 & 0.007684 & 0.007836 & 0.063719 & 0.008525 & 0.066768 & 0.061870 & 0.056833 \\
\hline $\mathrm{Fe}_{2} \mathrm{O}_{3}$ & 0.003153 & 0.040724 & 0.001978 & 0.028103 & 0.045183 & 0.044235 & 0.040990 & 0.001793 \\
\hline $\mathrm{Al}_{2} \mathrm{O}_{3}$ & 0.112294 & 0.012149 & 0.012391 & 0.012594 & 0.013479 & 0.013196 & 0.012228 & 0.011233 \\
\hline & 0.000255 & 0.002513 & 0.056391 & 0.025896 & 0.002788 & 0.040949 & 0.002530 & 0.002324 \\
\hline $\mathrm{Ag}_{2} \mathrm{O}$ & 0.000000 & 0.000000 & 0.000000 & 0.000000 & 0.000000 & 0.000000 & 0.000000 & 0.000000 \\
\hline $\mathrm{As}_{2} \mathrm{O}_{3}$ & 0.000000 & 0.000000 & 0.000000 & 0.000000 & 0.000000 & 0.000000 & 0.000000 & 0.000000 \\
\hline $\mathrm{BaO}$ & 0.000224 & 0.000145 & 0.000664 & 0.000150 & 0.000722 & 0.000157 & 0.000594 & 0.000134 \\
\hline CdO & 0.002008 & 0.001296 & 0.005946 & 0.001343 & 0.006468 & 0.001407 & 0.005320 & 0.001198 \\
\hline $\mathrm{Ce}_{2} \mathrm{O}_{3}$ & 0.000150 & 0.000097 & 0.000444 & 0.000100 & 0.000483 & 0.000105 & 0.000398 & 0.000089 \\
\hline $\mathrm{CoO}$ & 0.000000 & 0.000000 & 0.000000 & 0.000000 & 0.000000 & 0.000000 & 0.000000 & 0.000000 \\
\hline $\mathrm{Cr}_{2} \mathrm{O}_{3}$ & 0.000283 & 0.000183 & 0.000838 & 0.000189 & 0.000911 & 0.000198 & 0.000750 & 0.000169 \\
\hline & 0.000183 & 0.000118 & 0.000543 & 0.000123 & 0.000590 & 0.000128 & 0.000486 & 0.000109 \\
\hline CuO & 0.000649 & 0.000419 & 0.001923 & 0.000434 & 0.002091 & 0.000455 & 0.001720 & 0.000387 \\
\hline$F$ & 0.005426 & 0.003501 & 0.016068 & 0.003629 & 0.017479 & 0.003803 & 0.014377 & 0.003237 \\
\hline $\mathrm{K}_{2} \mathrm{O}$ & 0.000000 & 0.000000 & 0.000000 & 0.000000 & 0.000000 & 0.000000 & 0.000000 & 0.000000 \\
\hline $\mathrm{La}_{2} \mathrm{O}_{3}$ & 0.000659 & 0.000425 & 0.001953 & 0.000441 & 0.002124 & 0.000462 & 0.001747 & 0.000393 \\
\hline $\mathrm{MnO}$ & 0.000594 & 0.000383 & 0.001759 & 0.000397 & 0.001914 & 0.000416 & 0.001574 & 0.000354 \\
\hline $\mathrm{MoO}_{3}$ & 0.000716 & 0.000462 & 0.002121 & 0.000479 & 0.002307 & 0.000502 & 0.001898 & 0.000427 \\
\hline $\mathrm{Nd}_{2} \mathrm{O}_{3}$ & 0.001260 & 0.000813 & 0.003732 & 0.000843 & 0.004060 & 0.000883 & 0.003340 & 0.000752 \\
\hline $\mathrm{NiO}$ & 0.002647 & 0.001708 & 0.007839 & 0.001771 & 0.008528 & 0.001855 & 0.007014 & 0.001579 \\
\hline $\mathrm{P}_{2} \mathrm{O}_{5}$ & 0.000242 & 0.000156 & 0.000717 & 0.000162 & 0.000780 & 0.000170 & 0.000642 & 0.000144 \\
\hline $\mathrm{PoO}$ & 0.000000 & 0.000000 & 0.000000 & 0.000000 & 0.000000 & 0.000000 & 0.000000 & 0.000000 \\
\hline PdO & 0.000141 & 0.000091 & 0.000418 & 0.000094 & 0.000455 & 0.000099 & 0.000374 & 0.000084 \\
\hline $\mathrm{Pr}_{6} \mathrm{O}_{11}$ & 0.000034 & 0.000022 & 0.000100 & 0.000023 & 0.000108 & 0.000024 & 0.000089 & 0.000020 \\
\hline $\mathrm{Rb}_{2} \mathrm{O}$ & 0.000092 & 0.000060 & 0.000274 & 0.000062 & 0.000298 & 0.000065 & 0.000245 & 0.000055 \\
\hline $\mathrm{Rh}_{2} \mathrm{O}_{3}$ & 0.000068 & 0.000044 & 0.000202 & 0.000046 & 0.000219 & 0.000048 & 0.000180 & 0.000041 \\
\hline $\mathrm{RuO}_{2}$ & 0.000388 & 0.000250 & 0.001149 & 0.000260 & 0.001250 & 0.000272 & 0.001028 & 0.000232 \\
\hline $\mathrm{SO}_{3}$ & 0.001182 & 0.000763 & & 0.000791 & 0.003809 & 0.000829 & 0.003133 & 0.000705 \\
\hline & 0.000000 & 0.000000 & 0.000000 & 0.000000 & 0.000000 & 0.000000 & 0.000000 & 0.000000 \\
\hline & 0.000000 & 0.000000 & 0.000000 & 0.000000 & 0.000000 & 0.000000 & 0.000000 & 0.000000 \\
\hline $\mathrm{Sm}_{2} \mathrm{O}_{3}$ & 0.000050 & 0.000032 & 0.000147 & 0.000033 & 0.000160 & 0.000035 & 0.000131 & 0.000030 \\
\hline SrO & 0.000332 & 0.000214 & 0.000982 & 0.000222 & 0.001068 & 0.000232 & 0.000879 & 0.000198 \\
\hline $\mathrm{TeO}_{2}$ & 0.000000 & 0.000000 & 0.000000 & 0.000000 & 0.000000 & 0.000000 & 0.000000 & 0.000000 \\
\hline $\mathrm{TiO}_{2}$ & 0.000000 & 0.000000 & 0.000000 & 0.000000 & 0.000000 & 0.000000 & 0.000000 & 0.000000 \\
\hline $\mathrm{U}_{3} \mathrm{O}_{8}$ & 0.000000 & 0.000000 & 0.000000 & 0.000000 & 0.000000 & 0.000000 & 0.000000 & 0.000000 \\
\hline $\mathrm{WO}_{3}$ & 0.000000 & 0.000000 & 0.000000 & 0.000000 & 0.000000 & 0.000000 & 0.000000 & 0.000000 \\
\hline $\mathrm{Y}_{2} \mathrm{O}_{3}$ & 0.000077 & 0.000049 & 0.000227 & 0.000051 & 0.000246 & 0.000054 & 0.000203 & 0.000046 \\
\hline $\mathrm{ZnO}$ & 0.000000 & 0.000000 & 0.000000 & 0.000000 & 0.000000 & 0.000000 & 0.000000 & 0.000000 \\
\hline РСТ B & 0.278 & 14.871 & 9.512 & & & 0.764 & & 44.000 \\
\hline PCT Li & & & 6.221 & 0.698 & 0.684 & 0.672 & 8.064 & 19.969 \\
\hline PCT Na & 0.100 & 12.541 & 6.552 & 0.593 & 0.660 & 0.600 & 12.824 & 35.377 \\
\hline
\end{tabular}

* The glass names are those used in the reference from which the data were taken. 
Table A.4. CVS-II Phase 3 Glass Compositions (mole fractions) and PCT Normalized B, Li, and $\mathrm{Na}$ Releases $\left(\mathrm{g} / \mathrm{m}^{2}\right)$ (continued)

\begin{tabular}{|c|c|c|c|c|c|c|c|c|}
\hline Comp. & CVS2-83* & CVS2-84 & CVS2-85 & CVS2-86 & CVS2-87 & CVS2-88 & CVS2-89 & CVS2-90 \\
\hline $\mathrm{SiO}_{2}$ & 0.488745 & 0.486090 & 0.453040 & 0.523892 & 0.548474 & 0.480202 & 0.500662 & 0.578967 \\
\hline $\mathrm{B}_{2} \mathrm{O}_{3}$ & 0.081867 & 0.055322 & 0.053318 & 0.056946 & 0.075997 & 0.054093 & 0.114998 & 0.142766 \\
\hline $\mathrm{Na}_{2} \mathrm{O}$ & 0.174049 & 0.186420 & 0.179668 & 0.191892 & 0.192066 & 0.142076 & 0.198554 & 0.054322 \\
\hline $\mathrm{Li}_{2} \mathrm{O}$ & 0.140192 & 0.150371 & 0.144925 & 0.030072 & 0.039838 & 0.147031 & 0.022880 & 0.145520 \\
\hline $\mathrm{CaO}$ & 0.042747 & 0.005723 & 0.005516 & 0.047129 & 0.016156 & 0.044768 & 0.048765 & 0.005538 \\
\hline MgO & 0.007435 & 0.007963 & 0.030699 & 0.008197 & 0.008204 & 0.007786 & 0.008481 & 0.007706 \\
\hline $\mathrm{Fe}_{2} \mathrm{O}_{3}$ & $0: 001876$ & 0.042206 & 0.001937 & 0.002069 & 0.010353 & 0.009826 & 0.044953 & 0.039210 \\
\hline $\mathrm{Al}_{2} \mathrm{O}_{3}$ & 0.011756 & 0.012591 & 0.103147 & 0.110165 & 0.064019 & 0.064634 & 0.013411 & 0.012185 \\
\hline $\mathrm{ZrO}_{2}$ & 0.002432 & 0.041675 & 0.002510 & 0.002681 & 0.032901 & 0.038203 & 0.034900 & 0.002521 \\
\hline $\mathrm{Ag}_{2} \mathrm{O}$ & 0.000000 & 0.000000 & 0.000000 & 0.000000 & 0.000000 & 0.000000 & 0.000000 & 0.000000 \\
\hline $\mathrm{As}_{2} \mathrm{O}_{3}$ & 0.000000 & 0.000000 & 0.000000 & 0.000000 & 0.000000 & 0.000000 & 0.000000 & 0.000000 \\
\hline $\mathrm{BaO}^{\circ}$ & 0.000630 & 0.000150 & 0.000325 & 0.000347 & 0.000154 & 0.000147 & 0.000160 & 0.000145 \\
\hline CdO & 0.005641 & 0.001343 & 0.002912 & 0.003110 & 0.001383 & 0.001313 & 0.001430 & 0.001299 \\
\hline $\mathrm{Ce}_{2} \mathrm{O}_{3}$ & 0.000421 & 0.000100 & 0.000218 & 0.000232 & 0.000103 & 0.000098 & 0.000107 & 0.000097 \\
\hline $\mathrm{CoO}$ & 0.000000 & 0.000000 & 0.000000 & 0.000000 & 0.000000 & 0.000000 & 0.000000 & 0.000000 \\
\hline $\mathrm{C}_{\mathrm{T}_{2}} \mathrm{O}_{3}$ & 0.000795 & 0.000189 & 0.000410 & 0.000438 & 0.000195 & 0.000185 & 0.000202 & 0.000183 \\
\hline $\mathrm{Cs}_{2} \mathrm{O}$ & 0.000515 & 0.000123 & 0.000266 & 0.000284 & 0.000126 & 0.000120 & 0.000131 & 0.000119 \\
\hline CúO & 0.001824 & 0.000434 & 0.000941 & 0.001005 & 0.000447 & 0.000424 & 0.000462 & 0.000420 \\
\hline $\mathbf{F}$ & 0.015244 & 0.003628 & 0.007868 & 0.008403 & 0.003738 & 0.003548 & 0.003865 & 0.003511 \\
\hline $\mathrm{K}_{2} \mathrm{O}$ & 0.000000 & 0.000000 & 0.000000 & 0.000000 & 0.000000 & 0.000000 & 0.000000 & 0.000000 \\
\hline $\mathrm{La}_{2} \mathrm{O}_{3}$ & 0.001852 & 0.000441 & 0.000956 & 0.001021 & 0.000454 & 0.000431 & 0.000470 & 0.000427 \\
\hline $\mathrm{MnO}$ & 0.001669 & 0.000397 & 0.000861 & 0.000920 & 0.000409 & 0.000388 & 0.000423 & 0.000384 \\
\hline $\mathrm{MoO}_{3}$ & 0.002012 & 0.000479 & 0.001039 & 0.001109 & 0.000493 & 0.000468 & 0.000510 & 0.000464 \\
\hline $\mathrm{Nd}_{2} \mathrm{O}_{3}$ & 0.003541 & 0.000843 & 0.001828 & 0.001952 & 0.000868 & 0.000824 & 0.000898 & 0.000816 \\
\hline NiÓ & 0.007437 & 0.001770 & 0.003839 & 0.004100 & 0.001824 & 0.001731 & 0.001885 & 0.001713 \\
\hline $\mathrm{P}_{2} \mathrm{O}_{5}$ & 0.000680 & 0.000162 & 0.000351 & 0.000375 & 0.000167 & 0.000158 & 0.000172 & 0.000157 \\
\hline PSO & 0.000000 & 0.000000 & 0.000000 & 0.000000 & 0.000000 & 0.000000 & 0.000000 & 0.000000 \\
\hline PdO & 0.000397 & 0.000094 & 0.000205 & 0.000219 & 0.000097 & 0.000092 & 0.000101 & 0.000091 \\
\hline $\mathrm{Pr}_{6} \mathrm{O}_{11}$ & 0.000095 & 0.000022 & 0.000049 & 0.000052 & 0.000023 & 0.000022 & 0.000024 & 0.000022 \\
\hline $\mathrm{Rb}_{2} \mathrm{O}$ & 0.000260 & 0.000062 & 0.000134 & 0.000143 & 0.000064 & 0.000060 & 0.000066 & 0.000060 \\
\hline $\mathrm{Rh}_{2} \mathrm{O}_{3}$ & 0.000191 & 0.000046 & 0.000099 & 0.000105 & 0.000047 & 0.000045 & 0.000048 & 0.000044 \\
\hline $\mathrm{RuO}_{2}$ & 0.001090 & 0.000260 & 0.000563 & 0.000601 & 0.000267 & 0.000254 & 0.000276 & 0.000251 \\
\hline $\mathrm{SO}_{3}$ & 0.003321 & 0.000791 & 0.001714 & 0.001831 & 0.000815 & 0.000773 & 0.000842 & 0.000765 \\
\hline $\mathrm{Sb}_{2} \mathrm{O}_{3}$ & 0.000000 & 0.000000 & 0.000000 & 0.000000 & 0.000000 & 0.000000 & 0.000000 & 0.000000 \\
\hline $\mathrm{SeO}_{2}$ & 0.000000 & 0.000000 & 0.000000 & 0.000000 & 0.000000 & 0.000000 & 0.000000 & 0.000000 \\
\hline $\mathrm{Sm}_{2} \mathrm{O}_{3}$ & 0.000139 & 0.000033 & 0.000072 & 0.000077 & 0.000034 & 0.000032 & 0.000035 & 0.000032 \\
\hline SrO & 0.000932 & 0.000222 & 0.000481 & 0.000514 & 0.000228 & 0.000217 & 0.000236 & 0.000215 \\
\hline $\mathrm{TeO}_{2}$ & 0.000000 & 0.000000 & 0.000000 & 0.000000 & 0.000000 & 0.000000 & 0.000000 & 0.000000 \\
\hline $\mathrm{TiO}_{2}$ & 0.000000 & 0.000000 & 0.000000 & 0.000000 & 0.000000 & 0.000000 & 0.000000 & 0.000000 \\
\hline $\mathrm{U}_{3} \mathrm{O}_{8}$ & 0.000000 & 0.000000 & 0.000000 & 0.000000 & 0.000000 & 0.000000 & 0.000000 & 0.000000 \\
\hline $\mathrm{WO}_{3}$ & 0.000000 & 0.000000 & 0.000000 & 0.000000 & 0.000000 & 0.000000 & 0.000000 & 0.000000 \\
\hline $\mathrm{Y}_{2} \mathrm{O}_{3}$ & 0.000215 & 0.000051 & 0.000111 & 0.000119 & 0.000053 & 0.000050 & 0.000054 & 0.000050 \\
\hline $\mathrm{ZnO}$ & 0.000000 & 0.000000 & 0.000000 & 0.000000 & 0.000000 & 0.000000 & 0.000000 & 0.000000 \\
\hline РCT B & 34.656 & 12.460 & 0.456 & 0.115 & 0.178 & 0.308 & 1.716 & \\
\hline PCT Li & 19.228 & 6.682 & 0.422 & 0.091 & 0.131 & 0.376 & 1.236 & 3.764 \\
\hline $\mathrm{PCT} \mathrm{Na}$ & 27.890 & 8.236 & 0.749 & 0.254 & 0.286 & 0.473 & 1.348 & 3.481 \\
\hline
\end{tabular}

* The glass names are those used in the reference from which the data were taken. 
Table A.4. CVS-II Phase 3 Glass Compositions (mole fractions) and PCT Normalized $\mathrm{B}, \mathrm{Li}$, and $\mathrm{Na}$ Releases $\left(\mathrm{g} / \mathrm{m}^{2}\right)$ (continued)

\begin{tabular}{|c|c|c|c|c|c|c|c|c|}
\hline Comp. & CVS2-91* & CVS2-92 & CVS2-93 & CVS2-94 & CVS2-95 & CVS2-96 & CVS2-97 & CVS2-98 \\
\hline $\mathrm{SiO}_{2}$ & 0.589408 & 0.450082 & 0.468865 & 0.451923 & 0.504882 & 0.512371 & 0.578911 & 0.450027 \\
\hline & 0.145341 & 0.141253 & 0.122961 & 0.141831 & 0.098986 & 0.105186 & 0.098276 & 0.161186 \\
\hline $\mathrm{Na}_{2} \mathrm{O}$ & 0.107135 & 0.099164 & 0.132123 & 0.179225 & 0.167079 & 0.103769 & 0.118920 & 0.207746 \\
\hline $\mathrm{Liz}_{2} \mathrm{O}$ & 0.021164 & 0.143978 & 0.149987 & 0.108632 & 0.088762 & 0.080686 & 0.081492 & 0.079502 \\
\hline $\mathrm{CaO}$ & 0.005638 & 0.005480 & 0.011189 & 0.044018 & 0.012488 & 0.031444 & 0.009546 & 0.000000 \\
\hline $\mathrm{MgO}$ & 0.062762 & 0.060997 & 0.007943 & 0.007656 & 0.025531 & 0.057752 & 0.013606 & 0.000000 \\
\hline $\mathrm{Fe}_{2} \mathrm{O}_{3}$ & 0.001980 & 0.001924 & 0.039532 & 0.010473 & 0.036049 & 0.022807 & 0.029966 & 0.008063 \\
\hline $\mathrm{Al}_{2} \mathrm{O}_{3}$ & 0.012405 & 0.012056 & 0.012559 & 0.042549 & 0.022306 & 0.039998 & 0.015047 & 0.087640 \\
\hline $\mathrm{ZrO}_{2}$ & 0.002566 & 0.034915 & 0.002598 & 0.002504 & 0.020599 & 0.022325 & 0.020769 & 0.000000 \\
\hline $\mathrm{Ag}_{2} \mathrm{O}$ & 0.000000 & 0.000000 & 0.000000 & 0.000000 & 0.000000 & 0.000000 & 0.000000 & 0.000000 \\
\hline $\mathrm{As}_{2} \mathrm{O}_{3}$ & 0.000000 & 0.000000 & 0.000000 & 0.000000 & 0.000000 & 0.000000 & 0.000000 & 0.000000 \\
\hline $\mathrm{BaO}$ & 0.000664 & 0.000646 & 0.000673 & 0.000144 & 0.000000 & 0.000305 & 0.000423 & 0.000075 \\
\hline CdO & 0.005953 & 0.005785 & 0.006027 & 0.001291 & 0.000000 & 0.002729 & 0.003316 & 0.000673 \\
\hline $\mathrm{Ce}_{2} \mathrm{O}_{3}$ & 0.000445 & 0.000432 & 0.000450 & 0.000096 & 0.000000 & 0.000204 & 0.000032 & 0.000050 \\
\hline $\mathrm{COO}^{\circ}$ & 0.000000 & 0.000000 & 0.000000 & 0.000000 & 0.000000 & 0.000000 & 0.000000 & 0.000000 \\
\hline $\mathrm{Cr}_{2} \mathrm{O}_{3}$ & 0.000839 & 0.000815 & 0.000849 & 0.000182 & 0.000000 & 0.000385 & 0.000556 & 0.000095 \\
\hline $\mathrm{Cs}_{2} \mathrm{O}$ & 0.000543 & 0.000528 & 0.000550 & 0.000118 & 0.000000 & 0.000249 & 0.000349 & 0.000061 \\
\hline CuO & 0.001925 & 0.001871 & 0.001949 & 0.000417 & 0.000000 & 0.000883 & 1301 & 0.000218 \\
\hline$F$ & 0.016086 & 0.015634 & 0.016286 & 0.003488 & 0.000000 & 0.007376 & 0.006551 & 0.001820 \\
\hline $\mathrm{K}_{2} \mathrm{O}$ & 0.000000 & 0.000000 & 0.000000 & 0.000000 & 0.000263 & 0.000000 & 0.000000 & 0.000000 \\
\hline $\mathrm{La}_{2} \mathrm{O}_{3}$ & 0.001955 & 0.001900 & 0.001979 & 0.000424 & 0.000780 & 0.000896 & 0.003097 & 0.000221 \\
\hline Mno & 0.001761 & 0.001712 & 0.001783 & 0.000382 & 0.011706 & 0.000808 & 0.001191 & 0.000199 \\
\hline $\mathrm{MoO}_{3}$ & 0.002123 & 0.002064 & 0.002150 & 0.000460 & 0.000000 & 0.000974 & 0.001411 & 0.000240 \\
\hline $\mathrm{Nd}_{2} \mathrm{O}_{3}$ & 0.003737 & 0.003631 & 0.003783 & $0.000810^{\circ}$ & 0.000000 & 0.001713 & 0.001110 & 0.000423 \\
\hline Niō & 0.007848 & 0.007627 & 0.007945 & 0.001702 & 0.005061 & 0.003599 & 0.005262 & 0.000888 \\
\hline $\mathrm{P}_{2} \mathrm{O}_{5}$ & 0.000718 & 0.000698 & 0.000727 & 0.000156 & 0.000000 & 0.000329 & 0.000457 & 0.000081 \\
\hline PSO & 0.000000 & 0.000000 & 0.000000 & 0.000000 & 0.000000 & 0.000000 & 0.000000 & 0.000000 \\
\hline PdO & 0.000418 & 0.000407 & 0.000424 & 0.000091 & 0.000000 & 0.000192 & 0.000000 & 0.000047 \\
\hline $\mathrm{Pr}_{6} \mathrm{O}_{n}$ & 0.000100 & 0.000097 & 0.000101 & 0.000022 & 0.000000 & 0.000046 & 0.000121 & 0.000011 \\
\hline $\mathrm{Rb}_{2} \mathrm{O}$ & 0.000274 & 0.000266 & 0.000277 & 0.000059 & 0.000000 & 0.000126 & & 0.000031 \\
\hline $\mathrm{Rh}_{2}^{\mathrm{O}} \mathrm{O}_{3}$ & 0.000202 & 0.000196 & 0.000204 & 0.000044 & 0.000000 & 0.000093 & 0.000000 & 0.000023 \\
\hline $\mathrm{RuO}_{2}$ & 0.001151 & 0.001118 & 0.001165 & 0.000250 & 0.000000 & 0.000528 & 0.000743 & 0.000130 \\
\hline $\mathrm{SO}_{3}$ & 0.003505 & 0.003406 & 0.003548 & 0.000760 & 0.000000 & 0.001607 & 0.003601 & 0.000396 \\
\hline $\mathrm{Sb}_{2} \mathrm{O}_{3}$ & 0.000000 & 0.000000 & 0.000000 & 0.000000 & 0.000000 & 0.000000 & 0.000000 & 0.000000 \\
\hline $\mathrm{SeO}_{2}$ & 0.000000 & 0.000000 & 0.000000 & 0.000000 & 0.000000 & 0.000000 & 0.000000 & 0.000000 \\
\hline $\mathrm{Sm}_{2} \mathrm{O}_{3}$ & 0.000147 & 0.000143 & 0.000149 & 0.000032 & 0.000000 & 0.000067 & 0.000000 & 0.000017 \\
\hline Sro & 0.000983 & 0.000956 & 0.000995 & 0.000213 & 0.000000 & 0.000451 & 0.000000 & 0.000111 \\
\hline $\mathrm{TeO}_{2}$ & 0.000000 & 0.000000 & 0.000000 & 0.000000 & 0.000000 & 0.000000 & 0.000000 & 0.000000 \\
\hline $\mathrm{TiO}_{2}$ & 0.000000 & 0.000000 & 0.000000 & 0.000000 & 0.005508 & 0.000000 & 0.000000 & 0.000000 \\
\hline $\mathrm{U}_{3} \mathrm{O}_{2}$ & 0.000000 & 0.000000 & 0.000000 & 0.000000 & 0.000000 & 0.000000 & 0.000000 & 0.000000 \\
\hline $\mathrm{WO}_{3}$ & 0.000000 & 0.000000 & 0.000000 & 0.000000 & 0.000000 & 0.000000 & 0.000000 & 0.000000 \\
\hline $\mathrm{Y}_{2} \mathrm{O}_{3}$ & 0.000227 & 0.000220 & 0.000230 & 0.000049 & 0.000000 & 0.000104 & 0.000000 & 0.000026 \\
\hline & 0.000000 & 0.000000 & 0.000000 & 0.000000 & 0.000000 & 0.000000 & 0.003945 & 0.000000 \\
\hline РCT B & 8.642 & 18.590 & 13.227 & 4.070 & 9.9 & 0.4 & 1.4 & 4.52 \\
\hline PCT Li & & & & 3.066 & 4.271 & 0.429 & 1.052 & 3.497 \\
\hline $\mathrm{PCT} \mathrm{Na}$ & 6.738 & 11.792 & 10.010 & 3.350 & 7.259 & 0.410 & 1.134 & 2.796 \\
\hline
\end{tabular}

* The glass names are those used in the reference from which the data were taken. 
Table A.4. CVS-II Phase 3 Glass Compositions (mole fractions) and PCT Normalized $\mathrm{B}, \mathrm{Li}$, and $\mathrm{Na}$ Releases $\left(\mathrm{g} / \mathrm{m}^{2}\right)$ (continued)

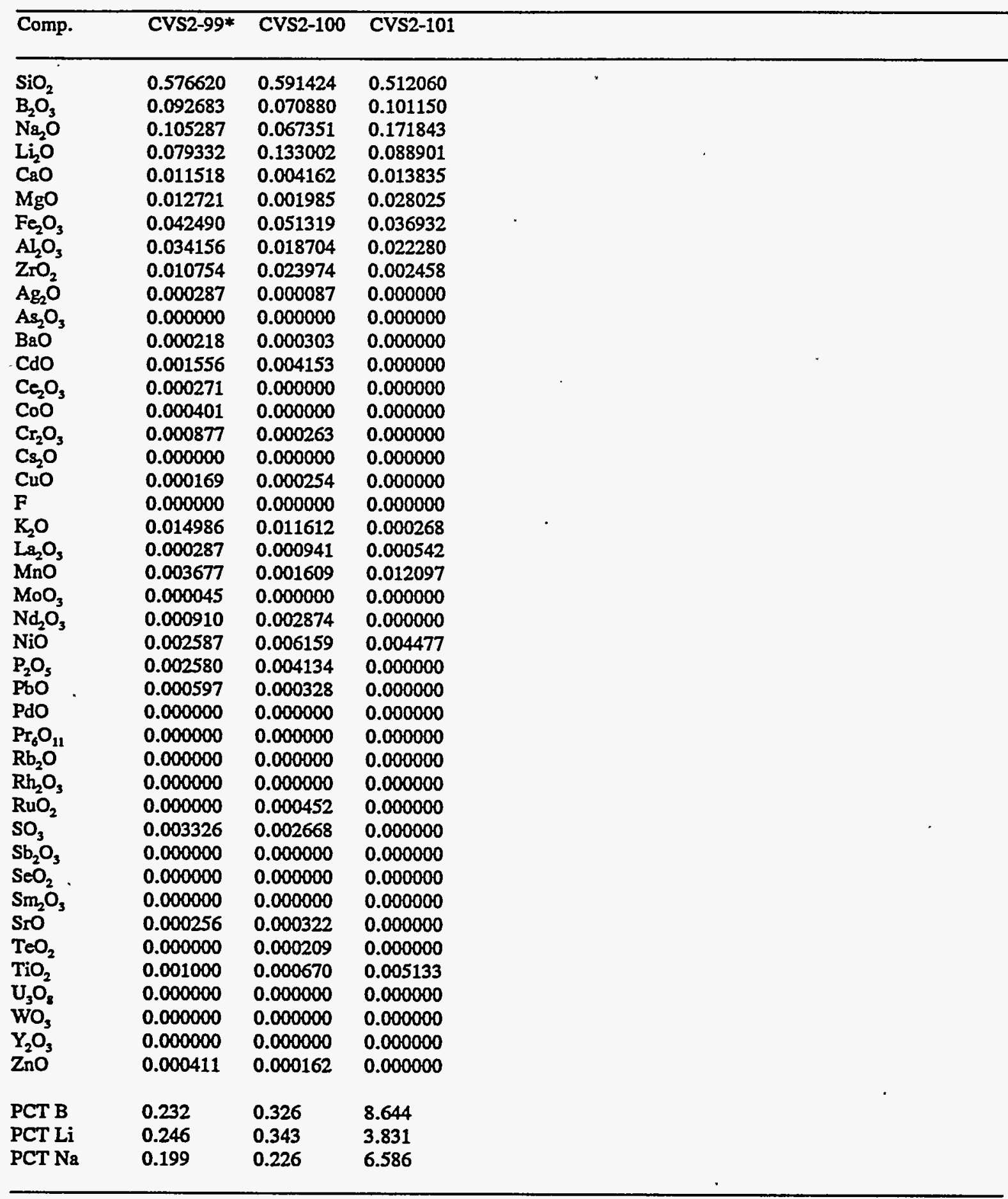

* The glass names are those used in the reference from which the data were taken. 
Table A.5. CVS-II Phase 4 Glass Compositions (mole fractions) and PCT Normalized $\mathrm{B}, \mathrm{Li}$, and Na Releases $\left(\mathrm{g} / \mathrm{m}^{2}\right)$

\begin{tabular}{|c|c|c|c|c|c|c|c|c|}
\hline Comp. & CVS2-102* & CVS2-103 & CVS2-104 & CVS2-105 & CVS2-106 & CVS2-107 & CVS2-108 & CVS2-109 \\
\hline $\mathrm{SiO}_{2}$ & 0.579488 & 0.453956 & 0.494661 & 0.534747 & 0.613270 & 0.612819 & 0.552342 & 0.521788 \\
\hline & 0.098382 & 0.127752 & 0.118210 & 0.108843 & 0.090480 & 0.046526 & 0.140604 & 0.188172 \\
\hline $\mathrm{Na}_{2} \mathrm{O}$ & 0.118063 & 0.153307 & 0.141889 & 0.130626 & 0.108578 & 0.124849 & 0.112532 & 0.106305 \\
\hline $\mathrm{Li}_{2} \mathrm{O}$ & 0.081628 & 0.105996 & 0.098094 & 0.090321 & 0.075070 & 0.086327 & 0.077811 & 0.073499 \\
\hline CäO & 0.009627 & 0.012503 & 0.011565 & 0.010652 & 0.008853 & 0.010177 & 0.009181 & 0.008667 \\
\hline $\mathrm{MgO}$ & 0.013556 & 0.017595 & 0.016289 & 0.015000 & 0.012462 & 0.014337 & 0.012920 & 0.012205 \\
\hline $\mathrm{Fe}_{2} \mathrm{O}_{3}$ & 0.029285 & 0.038030 & 0.035196 & 0.032401 & 0.026932 & 0.030968 & 0.027914 & 0.026370 \\
\hline $\mathrm{Al}_{2} \mathrm{O}_{3}$ & 0.014736 & 0.019137 & 0.017710 & 0.016303 & 0.013555 & 0.015585 & 0.014048 & 0.013266 \\
\hline $\mathrm{ZTO}_{2}$ & 0.020323 & 0.026390 & 0.024426 & 0.022483 & 0.018693 & 0.021492 & 0.019373 & 0.018297 \\
\hline $\mathrm{Ag}_{2} \mathrm{O}$ & 0.000000 & 0.000000 & 0.000000 & 0.000000 & 0.000000 & 0.000000 & 0.000000 & 0.000000 \\
\hline $\mathrm{As}_{2} \mathrm{O}_{3}$ & 0.000000 & 0.000000 & 0.000000 & 0.000000 & 0.000000 & 0.000000 & 0.000000 & 0.000000 \\
\hline $\mathrm{BaO}^{\circ}$ & 0.000450 & 0.000584 & 0.000540 & 0.000497 & 0.000413 & 0.000475 & 0.000428 & 0.000405 \\
\hline CdO & 0.004027 & 0.005229 & 0.004840 & 0.004456 & 0.003704 & 0.004259 & 0.003838 & 0.003626 \\
\hline $\mathrm{Ce}_{2} \mathrm{O}_{3}$ & 0.000301 & 0.000391 & 0.000362 & 0.000333 & 0.000277 & 0.000318 & 0.000287 & 0.000271 \\
\hline $\mathrm{COO}^{\circ}$ & 0.000000 & 0.000000 & 0.000000 & 0.000000 & 0.000000 & 0.000000 & 0.000000 & 0.000000 \\
\hline $\mathrm{Cr}_{2} \mathrm{O}_{3}$ & 0.000567 & 0.000737 & 0.000682 & 0.000628 & 0.000522 & 0.000600 & 0.000541 & 0.000511 \\
\hline $\mathrm{Cs}_{2} \mathrm{O}^{\circ}$ & 0.000368 & 0.000477 & 0.000442 & 0.000407 & 0.000338 & 0.000389 & 0.000350 & 0.000331 \\
\hline CuO & 0.001302 & 0.001691 & 0.001565 & 0.001441 & 0.001198 & 0.001377 & 0.001241 & 0.001172 \\
\hline $\mathbf{F}$ & 0.010883 & 0.014132 & 0.013080 & 0.012040 & 0.010009 & 0.011509 & 0.010373 & 0.009799 \\
\hline $\mathrm{K}_{2} \mathrm{O}$ & 0.000000 & 0.000000 & 0.000000 & 0.000000 & 0.000000 & 0.000000 & 0.000000 & 0.000000 \\
\hline $\mathrm{La}_{2} \mathrm{O}_{3}$ & 0.001322 & 0.001717 & 0.001589 & 0.001463 & 0.001216 & 0.001399 & 0.001260 & 0.001191 \\
\hline Mno & 0.001191 & 0.001547 & 0.001432 & 0.001318 & 0.001096 & 0.001260 & 0.001136 & 0.001073 \\
\hline $\mathrm{MoO}_{3}$ & 0.001437 & 0.001865 & 0.001727 & 0.001589 & 0.001321 & 0.001519 & 0.001369 & 0.001293 \\
\hline $\mathrm{Nd}_{2} \mathrm{O}_{3}$ & 0.002528 & 0.003283 & 0.003038 & 0.002797 & 0.002325 & 0.002673 & 0.002409 & 0.002276 \\
\hline Nió & 0.005309 & 0.006894 & 0.006381 & 0.005874 & 0.004883 & 0.005615 & 0.005060 & 0.004780 \\
\hline $\mathrm{P}_{2} \mathrm{O}_{3}$ & 0.000486 & 0.000631 & 0.000584 & 0.000537 & 0.000447 & 0.000514 & 0.000463 & 0.000437 \\
\hline Pbó & 0.000000 & 0.000000 & 0.000000 & 0.000000 & 0.000000 & 0.000000 & 0.000000 & 0.000000 \\
\hline PdO & 0.000283 & 0.000368 & 0.000340 & 0.000313 & 0.000260 & 0.000299 & 0.000270 & 0.000255 \\
\hline $\operatorname{Pr}_{6} \mathrm{O}_{11}$ & 0.000067 & 0.000088 & 0.000081 & 0.000075 & 0.000062 & 0.000071 & 0.000064 & 0.000061 \\
\hline $\mathrm{Rb}_{2} \mathrm{O}$ & 0.000185 & 0.000241 & 0.000223 & 0.000205 & 0.000170 & 0.000196 & 0.000177 & 0.000167 \\
\hline $\mathrm{Rh}_{2} \mathrm{O}_{3}$ & 0.000137 & 0.000177 & 0.000164 & 0.000151 & 0.000126 & 0.000144 & 0.000130 & 0.000123 \\
\hline $\mathrm{RuO}_{2}$ & 0.000778 & 0.001011 & 0.000936 & 0.000861 & 0.000716 & 0.000823 & 0.000742 & 0.000701 \\
\hline $\mathrm{SO}_{3}{ }^{2}$ & 0.002371 & 0.003079 & 0.002850 & 0.002623 & 0.002181 & 0.002508 & 0.002260 & 0.002135 \\
\hline $\mathrm{Sb}_{2} \mathrm{O}_{3}$ & 0.000000 & 0.000000 & 0.000000 & 0.000000 & 0.000000 & 0.000000 & 0.000000 & 0.000000 \\
\hline $\mathrm{SeO}_{2}$ & 0.000000 & 0.000000 & 0.000000 & 0.000000 & 0.000000 & 0.000000 & 0.000000 & 0.000000 \\
\hline $\mathrm{Sm}_{2} \mathrm{O}_{3}$ & 0.000099 & 0.000129 & 0.000119 & 0.000110 & 0.000091 & 0.000105 & 0.000095 & 0.000089 \\
\hline Sro & 0.000665 & 0.000864 & 0.000799 & 0.000736 & 0.000612 & 0.000703 & 0.000634 & 0.000599 \\
\hline $\mathrm{TeO}_{2}$ & 0.000000 & 0.000000 & 0.000000 & 0.000000 & 0.000000 & 0.000000 & 0.000000 & 0.000000 \\
\hline $\mathrm{TiO}_{2}$ & 0.000000 & 0.000000 & 0.000000 & 0.000000 & 0.000000 & 0.000000 & 0.000000 & 0.000000 \\
\hline $\mathrm{U}_{3} \mathrm{O}_{8}$ & 0.000000 & 0.000000 & 0.000000 & 0.000000 & 0.000000 & 0.000000 & 0.000000 & 0.000000 \\
\hline $\mathrm{WO}_{3}$ & 0.000000 & 0.000000 & 0.000000 & 0.000000 & 0.000000 & 0.000000 & 0.000000 & 0.000000 \\
\hline $\mathrm{Y}_{2} \mathrm{O}_{3}$ & 0.000153 & 0.000199 & 0.000184 & 0.000170 & 0.000141 & 0.000162 & 0.000146 & 0.000138 \\
\hline $\mathrm{ZnO}$ & 0.000000 & 0.000000 & 0.000000 & 0.000000 & 0.000000 & 0.000000 & 0.000000 & 0.000000 \\
\hline PCT B & 2.672 & 6.073 & 5.548 & 4.590 & 1.6 & 0.7 & 2.144 & 5.707 \\
\hline PCT Li & 1.921 & & & 3.132 & 1.348 & 0.662 & 1.646 & 4.494 \\
\hline PCT Na & 1.677 & 4.082 & 3.668 & 2.974 & 1.165 & 0.620 & 1.387 & 3.584 \\
\hline
\end{tabular}

* The glass names are those used in the reference from which the data were taken. 
Table A.5. CVS-II Phase 4 Glass Compositions (mole fractions) and PCT Normalized $\mathrm{B}, \mathrm{Li}$, and $\mathrm{Na}$ Releases $\left(\mathrm{g} / \mathrm{m}^{2}\right)$ (continued)

\begin{tabular}{|c|c|c|c|c|c|c|c|c|}
\hline Comp. & CVS2-110* & CVS2-111 & CVS2-112 & CVS2-113 & CVS2-114 & CVS2-115 & CVS2-116 & $\overline{\text { CVS2-117 }}$ \\
\hline $\mathrm{SiO}_{2}$ & 0.622469 & 0.553837 & 0.519827 & 0.616782 & 0.550790 & 0.538521 & 0.571568 & 0.587447 \\
\hline & 0.105683 & 0.094027 & 0.088255 & 0.104716 & 0.093510 & 0.091424 & 0.097040 & 0.099732 \\
\hline $\mathrm{Na}_{2} \mathrm{O}$ & 0.052656 & 0.157087 & 0.208876 & 0.125657 & 0.112216 & 0.109716 & 0.116448 & 0.119682 \\
\hline $\mathrm{Li}_{2} \mathrm{O}$ & 0.087680 & 0.078025 & 0.073218 & 0.022525 & 0.127108 & 0.146550 & 0.080518 & 0.082755 \\
\hline $\mathrm{CaO}$ & 0.010335 & 0.009201 & 0.008634 & 0.010250 & 0.009150 & 0.008947 & 0.023153 & 0.009759 \\
\hline $\mathrm{MgO}$ & 0.014559 & 0.012964 & 0.012158 & 0.014429 & 0.012885 & 0.012603 & 0.013369 & 0.000000 \\
\hline $\mathrm{Fe}_{2} \mathrm{O}_{3}$ & 0.031456 & 0.027988 & 0.026270 & 0.031169 & 0.027835 & 0.027214 & 0.028885 & 0.029688 \\
\hline $\mathrm{Al}_{2} \mathrm{O}_{3}$ & 0.015831 & 0.014081 & 0.013218 & 0.015685 & 0.014006 & 0.013695 & 0.014537 & 0.014941 \\
\hline $\mathrm{ZrO}_{2}$ & 0.021830 & 0.019422 & 0.018229 & .0 .021631 & 0.019317 & 0.018887 & 0.020048 & 0.020604 \\
\hline $\mathrm{Ag}_{2} \mathrm{O}$ & 0.000000 & 0.000000 & 0.000000 & 0.000000 & 0.000000 & 0.000000 & 0.000000 & 0.000000 \\
\hline $\mathrm{As}_{2} \mathrm{O}_{3}$ & 0.000000 & 0.000000 & 0.000000 & 0.000000 & 0.000000 & 0.000000 & 0.000000 & 0.000000 \\
\hline $\mathrm{BaO}$ & 0.000483 & 0.000430 & 0.000403 & 0.000478 & 0.000427 & 0.000418 & 0.000443 & 0.000456 \\
\hline $\mathrm{Bi}_{2} \mathrm{O}_{3}$ & 0.000000 & 0.000000 & 0.000000 & 0.000000 & 0.000000 & 0.000000 & 0.000000 & 0.000000 \\
\hline $\mathrm{CdO}$ & 0.004326 & 0.003849 & 0.003612 & 0.004286 & 0.003828 & 0.003742 & 0.003972 & 0.004082 \\
\hline $\mathrm{Ce}_{2} \mathrm{O}_{3}$ & 0.000323 & 0.000288 & 0.000270 & 0.000320 & 0.000286 & 0.000280 & 0.000297 & 0.000305 \\
\hline $\mathrm{CoO}$ & 0.000000 & 0.000000 & 0.000000 & 0.000000 & 0.000000 & 0.000000 & 0.000000 & 0.000000 \\
\hline $\mathrm{Cr}_{2} \mathrm{O}_{3}$ & 0.000610 & 0.000542 & 0.000509 & 0.000604 & 0.000539 & 0.000527 & 0.000560 & 0.000575 \\
\hline $\mathrm{Cs}_{2} \mathrm{O}$ & 0.000395 & 0.000351 & 0.000330 & 0.000391 & 0.000349 & 0.000342 & 0.000363 & 0.000373 \\
\hline CuO & 0.001399 & 0.001245 & 0.001168 & 0.001386 & 0.001238 & 0.001210 & 0.001284 & 0.001320 \\
\hline $\mathbf{F}$ & 0.011690 & 0.010401 & 0.009762 & 0.011583 & 0.010344 & 0.010113 & 0.010734 & 0.011032 \\
\hline $\mathrm{K}_{2} \mathrm{O}$ & 0.000000 & 0.000000 & 0.000000 & 0.000000 & 0.000000 & 0.000000 & 0.000000 & 0.000000 \\
\hline $\mathrm{La}_{2} \mathrm{O}_{3}$ & 0.001421 & 0.001264 & 0.001186 & 0.001408 & 0.001257 & 0.001229 & 0.001304 & 0.001341 \\
\hline $\mathrm{MnO}$ & 0.001280 & 0.001139 & 0.001069 & 0.001268 & 0.001132 & 0.001107 & 0.001175 & 0.001208 \\
\hline $\mathrm{MoO}_{3}$ & 0.001543 & 0.001373 & 0.001289 & 0.001529 & 0.001365 & 0.001335 & 0.001417 & 0.001456 \\
\hline $\mathrm{Nd}_{2} \mathrm{O}_{3}$ & 0.002715 & 0.002416 & 0.002268 & 0.002691 & 0.002403 & 0.002349 & 0.002493 & 0.002563 \\
\hline NiÓ & 0.005703 & 0.005075 & 0.004763 & 0.005651 & 0.005046 & 0.004934 & 0.005237 & 0.005382 \\
\hline $\mathrm{P}_{2} \mathrm{O}_{3}$ & 0.000522 & 0.000464 & 0.000436 & 0.000517 & 0.000462 & 0.000451 & 0.000479 & 0.000492 \\
\hline & 0.000000 & 0.000000 & 0.000000 & 0.000000 & 0.000000 & 0.000000 & 0.000000 & 0.000000 \\
\hline PdO & 0.000304 . & 0.000271 & 0.000254 & 0.000301 & 0.000269 & 0.000263 & 0.000279 & 0.000287 \\
\hline $\mathrm{Pr}_{6} \mathrm{O}_{11}$ & 0.000072 & 0.000064 & 0.000061 & 0.000072 & 0.000064 & 0.000063 & 0.000067 & 0.000068 \\
\hline $\mathrm{Rb}_{2} \mathrm{O}$ & 0.000199 & 0.000177 & 0.000166 & 0.000197 & 0.000176 & 0.000172 & 0.000183 & 0.000188 \\
\hline $\mathrm{Rh}_{2} \mathrm{O}_{3}$ & 0.000147 & 0.000131 & 0.000122 & 0.000145 & 0.000130 & 0.000127 & 0.000135 & 0.000138 \\
\hline $\mathrm{RuO}_{2}$ & 0.000836 & 0.000744 & 0.000698 & 0.000828 & 0.000740 & 0.000723 & 0.000768 & 0.000789 \\
\hline $\mathrm{SO}_{3}$ & 0.002547 & 0.002266 & 0.002127 & 0.002524 & 0.002254 & 0.002204 & 0.002339 & 0.002404 \\
\hline $\mathrm{Sb}_{2} \mathrm{O}_{3}$ & 0.000000 & 0.000000 & 0.000000 & 0.000000 & 0.000000 & 0.000000 & 0.000000 & 0.000000 \\
\hline & 0.000000 & 0.000000 & 0.000000 & 0.000000 & 0.000000 & 0.000000 & 0.000000 & 0.000000 \\
\hline $\mathrm{Sm}_{2} \mathrm{O}_{3}$ & 0.000107 & 0.000095 & 0.000089 & 0.000106 & 0.000094 & 0.000092 & 0.000098 & 0.000101 \\
\hline Sro & 0.000715 & 0.000636 & 0.000597 & 0.000708 & 0.000632 & 0.000618 & 0.000656 & 0.000674 \\
\hline $\mathrm{TeO}_{2}$ & 0.000000 & 0.000000 & 0.000000 & 0.000000 & 0.000000 & 0.000000 & 0.000000 & 0.000000 \\
\hline $\mathrm{TiO}_{2}$ & 0.000000 & 0.000000 & 0.000000 & 0.000000 & 0.000000 & 0.000000 & 0.000000 & 0.000000 \\
\hline $\mathrm{U}_{3} \mathrm{O}_{8}$ & 0.000000 & 0.000000 & 0.000000 & 0.000000 & 0.000000 & 0.000000 & 0.000000 & 0.000000 \\
\hline $\mathrm{WO}_{3}$ & 0.000000 & 0.000000 & 0.000000 & 0.000000 & 0.000000 & 0.000000 & 0.000000 & 0.000000 \\
\hline $\mathrm{Y}_{2} \mathrm{O}_{3}$ & 0.000165 & 0.000147 & 0.000138 & 0.000163 & 0.000146 & 0.000143 & 0.000151 & 0.000156 \\
\hline $\mathrm{ZnO}$ & 0.000000 & 0.000000 & 0.000000 & 0.000000 & 0.000000 & 0.000000 & 0.000000 & 0.000000 \\
\hline PCT B & 0.314 & 6.135 & 14.400 & 0.612 & 7.116 & 9.406 & 3.012 & 1.590 \\
\hline PCT Li & 0.402 & 3.894 & 7.794 & 0.630 & 4.928 & 5.837 & 2.203 & 1.337 \\
\hline PCT Na & 0.118 & 4.043 & 9.603 & 0.510 & 4.599 & 5.765 & 2.030 & 1.099 \\
\hline
\end{tabular}

* The glass names are those used in the reference from which the data were taken. 
Table A.5. CVS-II Phase 4 Glass Compositions (mole fractions) and PCT Normalized $\mathrm{B}, \mathrm{Li}$, and $\mathrm{Na}$ Releases $\left(\mathrm{g} / \mathrm{m}^{2}\right)$ (continued)

\begin{tabular}{|c|c|c|c|c|c|c|c|}
\hline Comp. & CVS2-118* & CVS2-119 & CVS2-120 & CVS2-121 & CVS2-122 & CVS2-123 & CVS2-124 \\
\hline $\mathrm{SiO}_{2}$ & 0.568631 & 0.588149 & 0.569204 & 0.549531 & 0.529092 & 0.578911 & 0.412660 \\
\hline $\mathrm{B}_{2} \mathrm{O}_{3}$ & 0.096540 & 0.099852 & 0.096636 & 0.093295 & 0.089825 & 0.098276 & 0.137413 \\
\hline $\mathrm{Na}_{2} \mathrm{O}$ & 0.115846 & 0.119827 & 0.115965 & 0.111955 & 0.107801 & 0.118920 & 0.164902 \\
\hline $\mathrm{Li}_{2} \mathrm{O}$ & 0.080095 & 0.082855 & 0.080185 & 0.077413 & 0.074533 & 0.081492 & 0.114012 \\
\hline $\mathrm{CaO}$ & 0.009443 & 0.009775 & 0.009454 & 0.009133 & 0.008787 & 0.009546 & 0.013446 \\
\hline MgO & 0.032046 & 0.013761 & 0.013318 & 0.012857 & 0.012379 & 0.013606 & 0.018934 \\
\hline $\mathrm{Fe}_{2} \mathrm{O}_{3}$ & 0.028737 & 0.029723 & 0.028766 & 0.027771 & 0.026739 & 0.029966 & 0.040904 \\
\hline $\mathrm{Al}_{2} \mathrm{O}_{3}$ & 0.014460 & 0.000000 & 0.032218 & 0.065664 & 0.100412 & 0.015047 & 0.020582 \\
\hline $\mathrm{ZrO}_{2}$ & 0.019942 & 0.020627 & 0.019962 & 0.019273 & 0.018556 & 0.020769 & 0.028386 \\
\hline $\mathrm{Ag}_{2} \mathrm{O}$ & 0.000000 & 0.000000 & 0.000000 & 0.000000 & 0.000000 & 0.000000 & 0.000000 \\
\hline $\mathrm{As}_{2} \mathrm{O}_{3}$ & 0.000000 & 0.000000 & 0.000000 & 0.000000 & 0.000000 & 0.000000 & 0.000000 \\
\hline $\mathrm{BaO}$ & 0.000441 & 0.000456 & 0.000442 & 0.000426 & 0.000410 & 0.000423 & 0.000628 \\
\hline CdO & 0.003952 & 0.004087 & 0.003956 & 0.003819 & 0.003677 & 0.003316 & 0.005625 \\
\hline $\mathrm{Ce}_{2} \mathrm{O}_{3}$ & 0.000295 & 0.000305 & 0.000296 & 0.000285 & 0.000275 & 0.000032 & 0.000420 \\
\hline $\mathrm{CoO}^{\circ}$ & 0.000000 & 0.000000 & 0.000000 & 0.000000 & 0.000000 & 0.000000 & 0.000000 \\
\hline $\mathrm{Cr}_{2} \mathrm{O}_{3}$ & 0.000557 & 0.000576 & 0.000557 & 0.000538 & 0.000518 & 0.000556 & 0.000793 \\
\hline $\mathrm{Cs}_{2} \mathrm{O}$ & 0.000361 & 0.000373 & 0.000361 & 0.000349 & 0.000336 & 0.000349 & 0.000513 \\
\hline CuO & $0.001278^{\prime}$ & 0.001322 & 0.001279 & 0.001235 & 0.001189 & 0.001301 & 0.001819 \\
\hline $\mathbf{F}$ & 0.010679 & 0.011046 & 0.010690 & 0.010320 & 0.009937 & 0.006551 & 0.015200 \\
\hline $\mathrm{K}_{2} \mathrm{O}$ & 0.000000 & 0.000000 & 0.000000 & 0.000000 & 0.000000 & 0.000000 & 0.000000 \\
\hline $\mathrm{La}_{2} \mathrm{O}_{3}$ & 0.001298 & 0.001342 & 0.001299 & 0.001254 & 0.001207 & 0.003097 & 0.001847 \\
\hline $\mathrm{MnO}$ & 0.001169 & 0.001209 & 0.001170 & 0.001130 & 0.001088 & 0.001191 & 0.001664 \\
\hline $\mathrm{MoO}_{3}$ & 0.001410 & 0.001458 & 0.001411 & 0.001362 & 0.001312 & 0.001411 & 0.002006 \\
\hline $\mathrm{Nd}_{2} \mathrm{O}_{3}$ & 0.002481 & 0.002566 & 0.002483 & 0.002397 & 0.002308 & 0.001110 & 0.003531 \\
\hline Nió & 0.005210 & 0.005389 & 0.005215 & 0.005035 & 0.004848 & 0.005262 & 0.007416 \\
\hline $\mathrm{P}_{2} \mathrm{O}_{3}$ & 0.000477 & 0.000493 & 0.000477 & 0.000460 & 0.000443 & 0.000457 & 0.000678 \\
\hline POOO & 0.000000 & 0.000000 & 0.000000 & 0.000000 & 0.000000 & 0.000000 & 0.000000 \\
\hline Pdo & 0.000278 & 0.000287 & 0.000278 & 0.000268 & 0.000259 & 0.000000 & 0.000395 \\
\hline $\mathrm{Pr}_{6} \mathrm{O}_{11}$ & 0.000066 & 0.000068 & 0.000066 & 0.000064 & 0.000062 & 0.000121 & 0.000094 \\
\hline $\mathrm{Rb}_{2} \mathrm{O}$ & 0.000182 & 0.000188 & 0.000182 & 0.000176 & 0.000169 & 0.000000 & 0.000259 \\
\hline $\mathrm{Rh}_{2} \mathrm{O}_{3}$ & 0.000134 & 0.000139 & 0.000134 & 0.000129 & 0.000125 & 0.000000 & 0.000191 \\
\hline $\mathrm{RuO}_{2}$ & 0.000764 & 0.000790 & 0.000765 & 0.000738 & 0.000711 & 0.000743 & 0.001087 \\
\hline $\mathrm{SO}_{3}$ & 0.002327 & 0.002407 & 0.002329 & 0.002249 & 0.002165 & 0.003601 & 0.003312 \\
\hline $\mathrm{Sb}_{2} \mathrm{O}_{3}$ & 0.000000 & 0.000000 & 0.000000 & 0.000000 & 0.000000 & 0.000000 & 0.000000 \\
\hline $\mathrm{SeO}_{2}$ & 0.000000 & 0.000000 & 0.000000 & 0.000000 & 0.000000 & 0.000000 & 0.000000 \\
\hline $\mathrm{Sm}_{2} \mathrm{O}_{3}$ & 0.000097 & 0.000101 & 0.000098 & 0.000094 & 0.000091 & 0.000000 & 0.000139 \\
\hline Sro & 0.000653 & 0.000675 & 0.000653 & 0.000631 & 0.000607 & 0.000000 & 0.000929 \\
\hline $\mathrm{TeO}_{2}$ & 0.000000 & 0.000000 & 0.000000 & 0.000000 & 0.000000 & 0.000000 & 0.000000 \\
\hline $\mathrm{TiO}_{2}^{2}$ & 0.000000 & 0.000000 & 0.000000 & 0.000000 & 0.000000 & 0.000000 & 0.000000 \\
\hline $\mathrm{U}_{3} \mathrm{O}_{8}$ & 0.000000 & 0.000000 & 0.000000 & 0.000000 & 0.000000 & 0.000000 & 0.000000 \\
\hline $\mathrm{Wo}_{3}$ & 0.000000 & 0.000000 & 0.000000 & 0.000000 & 0.000000 & 0.000000 & 0.000000 \\
\hline $\mathrm{Y}_{2} \mathrm{O}_{3}$ & 0.000151 & 0.000156 & 0.000151 & 0.000146 & 0.000140 & 0.000000 & 0.000214 \\
\hline $\mathrm{ZnO}^{\circ}$ & 0.000000 & 0.000000 & 0.000000 & 0.000000 & 0.000000 & 0.003945 & 0.000000 \\
\hline РСТ B & 3.630 & 3.803 & 0.291 & 0.199 & 0.193 & 1.473 & 5.567 \\
\hline PCT Li & 2.609 & 2.688 & 0.324 & 0.311 & 0.363 & 1.125 & 4.424 \\
\hline PCT $\mathrm{Na}$ & 2.394 & 2.574 & 0.231 & 0.137 & 0.089 & 1.075 & 4.293 \\
\hline
\end{tabular}

* The glass names are those used in the reference from which the data were taken. 
Table A.6. Ramsey Glass Compositions (mole fractions) and PCT Normalized $\mathrm{Si}, \mathrm{B}$, and $\mathrm{Na}$ Releases $\left(\mathrm{g} / \mathrm{m}^{2}\right)$

\begin{tabular}{|c|c|c|c|c|c|c|c|c|c|c|}
\hline$\# *$ & $\mathrm{SiO}_{2}$ & $\mathrm{Al}_{2} \mathrm{O}_{3}$ & $\mathrm{~B}_{2} \mathrm{O}_{3}$ & $\mathrm{Fe}_{2} \mathrm{O}_{3}$ & $\mathrm{FeO}$ & $\mathrm{CaO}$ & $\mathrm{Na}_{2} \mathrm{O}$ & PCT Si & РCT B & PCT Na \\
\hline 1 & 0.6049 & 0.1046 & 0.1407 & 0 & 0 & 0 & 0.1499 & 0.093 & 0.555 & 0.239 \\
\hline 2 & 0.5903 & 0.1038 & 0.0495 & 0 & 0 & 0.1035 & 0.1529 & 0.045 & 0.075 & 0.187 \\
\hline 4 & 0.6009 & 0.1107 & 0.0430 & 0 & 0 & 0 & 0.2454 & 0.187 & 0.368 & 0.685 \\
\hline 5 & 0.5887 & 0.0349 & 0.1365 & 0 & 0 & 0.0961 & 0.1438 & 0.179 & 0.361 & 0.364 \\
\hline 6 & 0.5857 & 0.0296 & 0.1312 & 0.0871 & 0.0256 & 0 & 0.1407 & 0.162 & 0.817 & 0.512 \\
\hline 7 & 0.5938 & 0.0062 & 0.1446 & 0 & 0 & 0 & 0.2555 & 15.029 & 29.834 & 25.044 \\
\hline 8 & 0.5800 & 0.0252 & 0.0473 & 0.0828 & 0.0290 & 0.0934 & 0.1424 & 0.108 & 0.257 & 0.597 \\
\hline 9 & 0.5953 & 0.0012 & 0.0491 & 0 & 0 & 0.1002 & 0.2543 & 16.036 & 38.709 & 27.827 \\
\hline 10 & 0.5777 & 0.0165 & 0.0409 & 0.0794 & 0.0294 & 0 & 0.2561 & 0.710 & 1.702 & 1.508 \\
\hline 11 & 0.4817 & 0.1084 & 0.1543 & 0 & 0 & 0.1040 & 0.1516 & 0.047 & 0.254 & 0.286 \\
\hline 13 & 0.5000 & 0.1198 & 0.1390 & 0 & 0 & 0 & 0.2412 & 0.085 & 2.122 & 1.133 \\
\hline 15 & 0.5036 & 0.1121 & 0.0453 & 0 & 0 & 0.0980 & 0.2410 & 0.126 & 0.174 & 0.762 \\
\hline 16 & 0.5046 & 0.1084 & 0.0440 & 0.0874 & 0.0225 & 0 & 0.2331 & 0.218 & 0.493 & 0.620 \\
\hline 17 & 0.4551 & 0.0810 & 0.1344 & 0.0715 & 0.0340 & 0.0896 & 0.1344 & 0.078 & 0.184 & 0.481 \\
\hline 18 & 0.4912 & 0.0057 & 0.1429 & 0 & 0 & 0.1026 & 0.2576 & 7.624 & 31.162 & 22.973 \\
\hline 19 & 0.4643 & 0.0260 & 0.1319 & 0.0901 & 0.0146 & 0 & 0.2730 & 0.367 & 5.016 & 2.621 \\
\hline 20 & 0.4876 & 0.0067 & 0.0496 & 0.0975 & 0.0133 & 0.0992 & 0.2461 & 0.361 & 0.770 & 1.748 \\
\hline 21 & 0.5684 & 0.0751 & 0.0861 & 0.0374 & 0.0080 & 0.0384 & 0.1865 & 0.126 & 0.275 & 0.378 \\
\hline 22 & 0.4694 & 0.1295 & 0.0974 & 0.0489 & 0.0166 & 0.0532 & 0.1850 & 0.084 & 0.165 & 0.240 \\
\hline 23 & 0.5047 & 0.1568 & 0.0774 & 0.0295 & 0.0162 & 0.0352 & 0.1802 & 0.086 & 0.152 & 0.205 \\
\hline 24 & 0.5302 & 0.0500 & 0.1036 & 0.0527 & 0.0166 & 0.0560 & 0.1909 & 0.128 & 0.436 & 0.531 \\
\hline 25 & 0.5212 & 0.0876 & 0.1357 & 0.0355 & 0.0111 & 0.0370 & 0.1720 & 0.103 & 0.417 & 0.353 \\
\hline 26 & 0.5553 & 0.0755 & 0.0432 & 0.0493 & 0.0244 & 0.0563 & 0.1960 & 0.123 & 0.254 & 0.472 \\
\hline 27 & 0.5112 & 0.0972 & 0.0795 & 0.0333 & 0.0122 & 0.0936 & 0.1731 & 0.058 & 0.137 & 0.277 \\
\hline 28 & 0.5412 & 0.1016 & 0.0938 & 0.0496 & 0.0212 & 0 & 0.1926 & 0.134 & 0.307 & 0.312 \\
\hline 29 & 0.5348 & 0.0817 & 0.0765 & 0.0784 & 0.0301 & 0.0345 & 0.1640 & 0.120 & 0.276 & 0.382 \\
\hline 30 & 0.5542 & 0.0673 & 0.1130 & 0 & 0 & 0.0631 & 0.2025 & 0.138 & 0.847 & 0.817 \\
\hline 31 & 0.5099 & 0.1002 & 0.0776 & 0.0346 & 0.0109 & 0.0368 & 0.2300 & 0.147 & 0.310 & 0.469 \\
\hline 32 & 0.5264 & 0.1130 & 0.0943 & 0.0442 & 0.0282 & 0.0541 & 0.1400 & 0.050 & 0.123 & 0.202 \\
\hline 33 & 0.5116 & 0.1234 & 0.0877 & 0.0372 & 0.0181 & 0.0430 & 0.1790 & 0.088 & 0.168 & 0.237 \\
\hline
\end{tabular}

* The glass numbers are those used in the reference from which the data were taken. Note that three mumbers are missing, so that there are only 30 glasses, not 33. 
Table A.7. WV87 Glass Compositions (mole fractions) and PCT Normalized B, Li, and Na Releases $\left(\mathrm{g} / \mathrm{m}^{2}\right)$

\begin{tabular}{|c|c|c|c|c|c|c|c|c|}
\hline Comp. & WVDG-15* & WVDG-16 & WVDG-17 & WVDG-18 & WVDG-19 & WVDG-20 & WVDG-21 & WVDG-22 \\
\hline $\mathrm{Al}_{2} \mathrm{O}_{3}$ & 0.068181 & 0.067416 & 0.066961 & 0.066586 & 0.091558 & 0.087620 & 0.091514 & 0.092402 \\
\hline $\mathrm{B}_{2} \mathrm{O}_{3}$ & 0.130854 & 0.132033 & 0.101710 & 0.109363 & 0.110520 & 0.111342 & 0.107674 & 0.125261 \\
\hline $\mathrm{BaO}$ & 0.001559 & 0.001521 & 0.001511 & 0.000514 & 0.001593 & 0.001476 & 0.000535 & 0.000541 \\
\hline $\mathrm{CaO}$ & 0.017562 & 0.017808 & 0.018462 & 0.006903 & 0.017831 & 0.017441 & 0.006650 & 0.006720 \\
\hline $\mathrm{Ce}_{2} \mathrm{O}_{3}$ & 0.002609 & 0.000805 & 0.002860 & 0.000812 & 0.000843 & 0.002862 & 0.000845 & 0.002890 \\
\hline $\mathrm{Cr}_{2} \mathrm{O}_{3}$ & 0.001143 & 0.000336 & 0.001239 & 0.000377 & 0.000352 & 0.001249 & 0.000393 & 0.001289 \\
\hline $\mathrm{Cs}_{2} \mathrm{O}^{\circ}$ & 0.000308 & 0.000103 & 0.000334 & 0.000102 & 0.000108 & 0.000337 & 0.000106 & 0.000348 \\
\hline $\mathrm{CuO}$ & 0.000182 & 0.000733 & 0.000182 & 0.000180 & 0.000672 & 0.000184 & 0.000656 & 0.000190 \\
\hline $\mathrm{Fe}_{2} \mathrm{O}_{3}$ & 0.039905 & 0.065822 & 0.040215 & 0.064826 & 0.067875 & 0.039993 & 0.068519 & 0.066219 \\
\hline $\mathrm{K}_{2} \mathrm{O}$ & 0.012608 & 0.010137 & 0.015911 & 0.013319 & 0.015964 & 0.017279 & 0.009740 & 0.014723 \\
\hline $\mathrm{Le}_{2} \mathrm{O}_{3}$ & 0.000156 & 0.000045 & 0.000178 & 0.000044 & 0.000047 & 0.000157 & 0.000046 & 0.000162 \\
\hline $\mathrm{Li}_{2} \mathrm{O}^{\circ}$ & 0.066159 & 0.046593 & 0.056697 & 0.058541 & 0.052878 & 0.049830 & 0.057660 & 0.045907 \\
\hline $\mathrm{MgO}$ & 0.000000 & 0.022065 & 0.023173 & 0.007827 & 0.024242 & 0.025715 & 0.009808 & 0.008976 \\
\hline $\mathrm{MnO}$ & 0.020907 & 0.018698 & 0.020737 & 0.004453 & 0.004390 & 0.004366 & 0.021449 & 0.021327 \\
\hline $\mathrm{MoO}_{3}$ & 0.000352 & 0.000101 & 0.000402 & 0.000100 & 0.000106 & 0.000355 & 0.000104 & 0.000367 \\
\hline $\mathrm{Na}_{2} \mathrm{O}$ & 0.118824 & 0.101615 & 0.123475 & 0.128857 & 0.121679 & 0.101631 & 0.125879 & 0.101056 \\
\hline $\mathrm{Nd}_{2} \mathrm{O}_{3}$ & 0.000517 & 0.000152 & 0.000559 & 0.000170 & 0.000159 & 0.000564 & 0.000177 & 0.000582 \\
\hline $\mathrm{NiO}$ & 0.005138 & 0.001561 & 0.005622 & 0.001632 & 0.001635 & 0.005667 & 0.001697 & 0.005751 \\
\hline $\mathrm{P}_{2} \mathrm{O}_{3}$ & 0.006785 & 0.016587 & 0.006325 & 0.007829 & 0.007260 & 0.017175 & 0.017761 & 0.007593 \\
\hline $\mathrm{Pr}_{6} \mathrm{O}_{11}$ & 0.000050 & 0.000014 & 0.000057 & 0.000014 & 0.000015 & 0.000050 & 0.000015 & 0.000052 \\
\hline $\mathrm{SO}_{3}$ & 0.003618 & 0.001093 & 0.003889 & 0.001164 & 0.001144 & 0.003920 & 0.001118 & 0.003954 \\
\hline $\mathrm{SiO}_{2}$ & 0.479557 & 0.484669 & 0.476819 & 0.515456 & 0.457473 & 0.485304 & 0.466014 & 0.468904 \\
\hline $\mathrm{Sm}_{2} \mathrm{O}_{3}$ & 0.000062 & 0.000021 & 0.000083 & 0.000021 & 0.000022 & 0.000084 & 0.000021 & 0.000086 \\
\hline sro & 0.000210 & 0.000070 & 0.000279 & 0.000069 & 0.000074 & 0.000282 & 0.000072 & 0.000291 \\
\hline $\mathrm{ThO}_{2}$ & 0.004553 & 0.004417 & 0.012860 & 0.005322 & 0.015149 & 0.005169 & 0.004492 & 0.004996 \\
\hline $\mathrm{TiO}_{2}$ & 0.013051 & 0.004014 & 0.014317 & 0.004127 & 0.004204 & 0.014342 & 0.004294 & 0.014527 \\
\hline $\mathrm{U}_{3} \mathrm{O}_{8}$ & 0.000822 & 0.000117 & 0.000072 & 0.000080 & 0.000876 & 0.000856 & 0.000847 & 0.000102 \\
\hline $\mathrm{Y}_{2} \mathrm{O}_{3}$ & 0.000096 & 0.000032 & 0.000128 & 0.000032 & 0.000034 & 0.000129 & 0.000033 & 0.000134 \\
\hline $\mathrm{ZnO}$ & 0.000178 & 0.000179 & 0.000534 & 0.000000 & 0.000000 & 0.000179 & 0.000550 & 0.000185 \\
\hline $\mathrm{ZO}_{2}$ & 0.004055 & 0.001242 & 0.004407 & 0.001280 & 0.001301 & 0.004443 & 0.001332 & 0.004465 \\
\hline PCT B & 0.296 & 0.189 & 0.292 & 0.225 & 0.214 & 0.160 & 0.225 & 0.210 \\
\hline PCT Li & 0.300 & 0.235 & 0.292 & 0.269 & 0.282 & 0.242 & 0.252 & 0.300 \\
\hline PCT $\mathrm{Na}$ & 0.264 & 0.165 & 0.273 & 0.219 & 0.207 & 0.139 & 0.222 & 0.179 \\
\hline
\end{tabular}

* The glass numbers are those used in the reference from which the data were taken. 
Table A.7. WV87 Glass Compositions (mole fractions)

and PCT Normalized $\mathrm{B}, \mathrm{Li}$, and $\mathrm{Na}$ Releases $\left(\mathrm{g} / \mathrm{m}^{2}\right)$ (continued)

\begin{tabular}{|c|c|c|c|c|c|c|c|c|}
\hline Comp. & WVDG-23* & WVDG-24 & WVDG-25 & WVDG-26 & WVDG-27 & WVDG-28 & WVDG-29 & WVDG-30 \\
\hline $\mathrm{Al}_{2} \mathrm{O}_{3}$ & 0.070257 & 0.086353 & 0.065548 & 0.086082 & 0.088273 & 0.069246 & 0.063024 & 0.072232 \\
\hline $\mathrm{B}_{2} \mathrm{O}_{3}$ & 0.136321 & 0.135709 & 0.112516 & 0.137478 & 0.133639 & 0.111683 & 0.113888 & 0.124804 \\
\hline $\mathrm{BaO}$ & 0.000546 & 0.000513 & 0.000469 & 0.001400 & 0.000531 & 0.000523 & 0.001445 & 0.000954 \\
\hline $\mathrm{CaO}$ & 0.006780 & 0.006117 & 0.006407 & 0.017092 & 0.006462 & 0.006243 & 0.016423 & 0.010955 \\
\hline $\mathrm{Ce}_{2} \mathrm{O}_{3}$ & 0.000928 & 0.000830 & 0.000919 & 0.000935 & 0.003094 & 0.002924 & 0.000885 & 0.001700 \\
\hline $\mathrm{Cr}_{2} \mathrm{O}_{3}$ & 0.000400 & 0.000376 & 0.000378 & 0.000424 & 0.001362 & 0.001296 & 0.000364 & 0.000770 \\
\hline $\mathrm{Cs}_{2} \mathrm{O}$ & 0.000108 & 0.000101 & 0.000102 & 0.000102 & 0.000367 & 0.000336 & 0.000098 & 0.000208 \\
\hline $\mathrm{CuO}$ & 0.000000 & 0.000180 & 0.000181 & 0.000270 & 0.000744 & 0.000734 & 0.000174 & 0.000368 \\
\hline $\mathrm{Fe}_{2} \mathrm{O}_{3}$ & 0.067811 & 0.038800 & 0.039016 & 0.038836 & 0.040199 & 0.055311 & 0.037899 & 0.052577 \\
\hline $\mathrm{K}_{2} \mathrm{O}$ & 0.015016 & 0.019119 & 0.015411 & 0.020048 & 0.019236 & 0.010453 & 0.013526 & 0.014053 \\
\hline $\mathrm{La}_{2} \mathrm{O}_{3}$ & 0.000047 & 0.000044 & 0.000044 & 0.000066 & 0.000182 & 0.000179 & 0.000043 & 0.000090 \\
\hline $\mathrm{Li}_{2} \mathrm{O}$ & 0.045808 & 0.049029 & 0.048821 & 0.047877 & 0.056430 & 0.050039 & 0.059556 & 0.051399 \\
\hline $\mathrm{MgO}$ & 0.010189 & 0.009930 & 0.009450 & 0.023960 & 0.011377 & 0.011220 & 0.023366 & 0.018328 \\
\hline $\mathrm{MnO}$ & 0.004461 & 0.020058 & 0.019839 & 0.004525 & 0.004339 & 0.004363 & 0.003983 & 0.010095 \\
\hline $\mathrm{MoO}_{3}$ & 0.000106 & 0.000099 & 0.000100 & 0.000149 & 0.000411 & 0.000405 & 0.000096 & 0.000203 \\
\hline $\mathrm{Na}_{2} \mathrm{O}$ & 0.100978 & 0.110234 & 0.105397 & 0.104911 & 0.120995 & 0.104266 & 0.119993 & 0.113637 \\
\hline $\mathrm{Nd}_{2} \mathrm{O}_{3}$ & 0.000181 & 0.000170 & 0.000171 & 0.000191 & 0.000615 & 0.000585 & 0.000165 & 0.000348 \\
\hline $\mathrm{NiO}$ & 0.001832 & 0.001626 & 0.001828 & 0.001819 & 0.006138 & 0.005761 & 0.001761 & 0.003427 \\
\hline $\mathrm{P}_{2} \mathrm{O}_{3}$ & 0.017679 & 0.007099 & 0.015847 & 0.008164 & 0.018132 & 0.007862 & 0.015952 & 0.012469 \\
\hline $\mathrm{Pr}_{6} \mathrm{O}_{11}$ & 0.000015 & 0.000014 & 0.000014 & 0.000021 & 0.000058 & 0.000057 & 0.000014 & 0.000029 \\
\hline $\mathrm{SO}_{3}$ & 0.001330 & 0.001160 & 0.001257 & 0.001251 & 0.004249 & 0.004009 & 0.001211 & 0.002375 \\
\hline $\mathrm{SiO}_{2}$ & 0.497523 & 0.500751 & 0.538222 & 0.487522 & 0.451977 & 0.526601 & 0.514930 & 0.487625 \\
\hline $\mathrm{Sm}_{2} \mathrm{O}_{3}$ & 0.000022 & 0.000020 & 0.000021 & 0.000021 & 0.000085 & 0.000084 & 0.000020 & 0.000042 \\
\hline SrO & 0.000073 & 0.000069 & 0.000069 & 0.000069 & 0.000285 & 0.000282 & 0.000067 & 0.000141 \\
\hline $\mathrm{ThO}_{2}$ & 0.014256 & 0.005089 & 0.011867 & 0.010295 & 0.009691 & 0.004751 & 0.005062 & 0.009030 \\
\hline $\mathrm{TiO}_{2}$ & 0.004759 & 0.004204 & 0.004587 & 0.004745 & 0.015550 & 0.014697 & 0.004420 & 0.008604 \\
\hline $\mathrm{U}_{3} \mathrm{O}_{8}$ & 0.000873 & 0.000820 & 0.000089 & 0.000088 & 0.000100 & 0.000864 & 0.000085 & 0.000533 \\
\hline & 0.000034 & 0.000032 & 0.000032 & 0.000032 & 0.000131 & 0.000129 & 0.000031 & 0.000065 \\
\hline $\mathrm{ZnO}$ & 0.000187 & 0.000176 & 0.000000 & 0.000176 & 0.000545 & 0.000538 & 0.000170 & 0.000270 \\
\hline $\mathrm{ZrO}_{2}$ & 0.001481 & 0.001276 & 0.001400 & 0.001451 & 0.004802 & 0.004558 & 0.001349 & 0.002671 \\
\hline PCT B & 0.253 & 0.213 & 0.188 & 0.185 & 0.309 & 0.201 & 0.217 & 0.209 \\
\hline PCT Li & 0.307 & 0.286 & 0.253 & 0.257 & 0.327 & 0.289 & $0.255^{* *}$ & $0.270 * *$ \\
\hline $\mathrm{PCT} \mathrm{Na}$ & 0.187 & 0.173 & 0.164 & 0.156 & 0.243 & 0.169 & 0.214 & 0.196 \\
\hline
\end{tabular}

* The glass numbers are those used in the reference from which the data were taken.

** Approximated from Figure 4.4 of Ref. 8 due to errors for these values in Table 4.3 of Ref. 8 . 
Table A.8. WV88 Glass Compositions (mole fractions) and PCT Normalized $\mathrm{B}, \mathrm{Li}$, and $\mathrm{Na}$ Releases $\left(\mathrm{g} / \mathrm{m}^{2}\right)$

\begin{tabular}{|c|c|c|c|c|c|c|c|c|}
\hline Comp. & WVDG-33* & WVDG-34 & WVDG-35 & WVDG-36 & WVDG-37 & WVDG-38 & WVDG-39 & WVDG-40 \\
\hline $\mathrm{Al}_{2} \mathrm{O}_{3}$ & 0.057810 & 0.036690 & 0.072311 & 0.042298 & 0.069628 & 0.073673 & 0.070996 & 0.043326 \\
\hline & 0.093011 & 0.112355 & 0.075571 & 0.117582 & 0.079496 & 0.079871 & 0.116084 & 0.078458 \\
\hline $\mathrm{BaO}$ & 0.000281 & 0.000142 & 0.000480 & 0.000099 & 0.000093 & 0.000096 & 0.000423 & 0.000473 \\
\hline $\mathrm{CaO}$ & 0.005115 & 0.002323 & 0.009185 & 0.002162 & 0.001776 & 0.002093 & 0.008477 & 0.009313 \\
\hline $\mathrm{Ce}_{2} \mathrm{O}_{3}$ & 0.001396 & 0.004015 & 0.000684 & 0.004139 & 0.003844 & 0.004113 & 0.001067 & 0.000885 \\
\hline $\mathrm{Cr}_{2} \mathrm{O}_{3}$ & 0.000613 & 0.001761 & 0.000290 & 0.001845 & 0.001685 & 0.001786 & 0.000474 & 0.000382 \\
\hline $\mathrm{Cs}_{2} \mathrm{O}$ & 0.000178 & 0.000488 & 0.000078 & 0.000484 & 0.000454 & 0.000495 & 0.000128 & 0.000103 \\
\hline CuO & 0.000361 & 0.001001 & 0.000185 & 0.000953 & 0.000894 & 0.001014 & 0.000272 & 0.000182 \\
\hline $\mathrm{Fe}_{2} \mathrm{O}_{3}$ & 0.051415 & 0.044269 & 0.069024 & 0.069581 & 0.043521 & 0.044421 & 0.041857 & 0.069725 \\
\hline $\mathrm{K}_{2} \mathrm{O}$ & 0.026187 & 0.027808 & 0.028981 & 0.020438 & 0.029452 & 0.025388 & 0.027681 & 0.024411 \\
\hline $\mathrm{La}_{2} \mathrm{O}_{3}$ & 0.000088 & 0.000244 & 0.000045 & 0.000233 & 0.000218 & 0.000248 & 0.000066 & 0.000045 \\
\hline $\mathrm{Li}_{2} \mathrm{O}$ & 0.069833 & 0.074341 & 0.077322 & 0.054535 & 0.078560 & 0.067756 & 0.073761 & 0.065300 \\
\hline $\mathrm{MgO}$ & 0.017436 & 0.008079 & 0.031767 & 0.007522 & 0.006354 & 0.007098 & 0.029309 & 0.032036 \\
\hline $\mathrm{MnO}$ & 0.010558 & 0.016480 & 0.016505 & 0.001046 & 0.001064 & 0.016623 & 0.000911 & 0.001001 \\
\hline $\mathrm{MoO}_{3}$ & 0.000199 & 0.000553 & 0.000102 & 0.000527 & 0.000494 & 0.000561 & 0.000150 & 0.000101 \\
\hline $\mathrm{Na}_{2} \mathrm{O}$ & 0.121598 & 0.129239 & 0.134630 & 0.094775 & 0.136696 & 0.117766 & 0.128301 & 0.113291 \\
\hline $\mathrm{Nd}_{2} \mathrm{O}_{3}$ & 0.000277 & 0.000796 & 0.000131 & 0.000833 & 0.000761 & 0.000807 & 0.000214 & 0.000172 \\
\hline NiO & 0.002784 & 0.007943 & 0.001379 & 0.008219 & 0.007618 & 0.008151 & 0.002121 & 0.001748 \\
\hline $\mathrm{P}_{2} \mathrm{O}_{3}$ & 0.012478 & 0.009533 & 0.000311 & 0.003150 & 0.002506 & 0.003101 & 0.019333 & 0.003066 \\
\hline $\mathrm{Pr}_{8} \mathrm{O}_{11}$ & 0.000028 & 0.000078 & 0.000014 & 0.000074 & 0.000070 & 0.000079 & 0.000021 & 0.000014 \\
\hline $\mathrm{SO}_{3}$ & 0.001881 & 0.005513 & 0.000919 & 0.005680 & 0.005331 & 0.005589 & 0.001440 & 0.001178 \\
\hline $\mathrm{SiO}_{2}$ & 0.507219 & 0.481712 & 0.465367 & 0.518464 & 0.497252 & 0.499348 & 0.455540 & 0.535544 \\
\hline $\mathrm{Sm}_{2} \mathrm{O}_{3}$ & 0.000041 & 0.000104 & 0.000021 & 0.000109 & 0.000102 & 0.000105 & 0.000021 & 0.000021 \\
\hline Sro & 0.000138 & 0.000349 & 0.000071 & 0.000366 & 0.000343 & 0.000354 & 0.000070 & 0.000070 \\
\hline $\mathrm{ThO}_{2}$ & 0.009152 & 0.005262 & 0.007747 & 0.016678 & 0.005631 & 0.011474 & 0.013940 & 0.011511 \\
\hline $\mathrm{TiO}_{2}$ & 0.007000 & 0.020195 & 0.003500 & 0.020775 & 0.019231 & 0.020658 & 0.005409 & 0.004449 \\
\hline $\mathrm{U}_{3} \mathrm{O}_{8}$ & 0.000443 & 0.001635 & 0.002180 & 0.000122 & 0.000123 & 0.000081 & 0.000089 & 0.001630 \\
\hline $\mathrm{Y}_{2} \mathrm{O}_{3}$ & 0.000064 & 0.000160 & 0.000033 & 0.000168 & 0.000158 & 0.000162 & 0.000032 & 0.000032 \\
\hline $\mathrm{ZnO}$ & 0.000264 & 0.000711 & 0.000090 & 0.000745 & 0.000699 & 0.000721 & 0.000177 & 0.000178 \\
\hline $\mathrm{ZrO}_{2}$ & 0.002153 & 0.006225 & 0.001075 & 0.006397 & 0.005946 & 0.006370 & 0.001637 & 0.001354 \\
\hline РCT B & 0.247 & 0.417 & 0.400 & 0.244 & 0.231 & 0.197 & 0.271 & 0.305 \\
\hline PCT Li & 0.263 & 0.388 & 0.394 & 0.251 & 0.267 & 0.240 & 0.249 & 0.305 \\
\hline PCT Na & 0.245 & 0.363 & 0.399 & 0.164 & 0.267 & 0.197 & 0.247 & 0.289 \\
\hline
\end{tabular}

* The glass numbers are those used in the reference from which the data were taken. 
Table A.8. WV88 Glass Compositions (mole fractions) and PCT Normalized $\mathrm{B}, \mathrm{Li}$, and $\mathrm{Na}$ Releases $\left(\mathrm{g} / \mathrm{m}^{2}\right)$ (continued)

\begin{tabular}{|c|c|c|c|c|c|c|c|c|}
\hline Comp. & WVDG-41* & WVDG-42 & WVDG-43 & WVDG-44 & WVDG-45 & WVDG-46 & WVDG-47 & WVDG-48 \\
\hline $\mathrm{Al}_{2} \mathrm{O}_{3}$ & 0.041905 & 0.039679 & 0.072406 & 0.060784 & 0.073694 & 0.047064 & 0.040476 & 0.068960 \\
\hline & 0.075265 & 0.070678 & 0.131287 & 0.123049 & 0.077274 & 0.120428 & 0.116789 & 0.113100 \\
\hline $\mathrm{BaO}$ & 0.000141 & 0.000475 & 0.000144 & 0.000146 & 0.000096 & 0.000447 & 0.000095 & 0.000486 \\
\hline $\mathrm{CaO}$ & 0.002574 & 0.008839 & 0.002622 & 0.002524 & 0.002100 & 0.008312 & 0.002069 & 0.009041 \\
\hline $\mathrm{Ce}_{2} \mathrm{O}_{3}$ & 0.001006 & 0.003918 & 0.000876 & 0.000866 & 0.000877 & 0.000737 & 0.000759 & 0.004440 \\
\hline $\mathrm{Cr}_{2} \mathrm{O}_{3}$ & 0.000427 & 0.001727 & 0.000387 & 0.000392 & 0.000387 & 0.000316 & 0.000334 & 0.001962 \\
\hline $\mathrm{Cs}_{2} \mathrm{O}$ & 0.000128 & 0.000466 & 0.000104 & 0.000106 & 0.000104 & 0.000097 & 0.000103 & 0.000529 \\
\hline CuO & 0.000272 & 0.000916 & 0.000185 & 0.000187 & 0.000185 & 0.000172 & 0.000182 & 0.001031 \\
\hline $\mathrm{Fe}_{2} \mathrm{O}_{3}$ & 0.042845 & 0.043502 & 0.043882 & 0.073748 & 0.071477 & 0.044602 & 0.067856 & 0.043143 \\
\hline $\mathrm{K}_{2} \mathrm{O}$ & 0.028733 & 0.026854 & 0.020921 & 0.027915 & 0.030001 & 0.024526 & 0.020559 & 0.021214 \\
\hline $\mathrm{La}_{2} \mathrm{O}_{3}$ & 0.000066 & 0.000224 & 0.000045 & 0.000046 & 0.000045 & 0.000042 & 0.000045 & 0.000252 \\
\hline $\mathrm{Li}_{2}^{2} \mathrm{O}$ & 0.076566 & 0.071722 & 0.055860 & 0.074537 & 0.080043 & 0.065613 & 0.054856 & 0.056643 \\
\hline $\mathrm{MgO}$ & 0.009133 & 0.030204 & 0.008940 & 0.008871 & 0.007304 & 0.028404 & 0.007198 & 0.030895 \\
\hline $\mathrm{MnO}$ & 0.000996 & 0.016351 & 0.016409 & 0.001114 & 0.017015 & 0.015455 & 0.016185 & 0.001029 \\
\hline $\mathrm{MoO}_{3}$ & 0.000150 & 0.000506 & 0.000102 & 0.000104 & 0.000102 & 0.000095 & 0.000101 & 0.000570 \\
\hline $\mathrm{Na}_{2} \mathrm{O}$ & 0.133217 & 0.124671 & 0.096929 & 0.129682 & 0.139281 & 0.113924 & 0.095726 & 0.098408 \\
\hline $\mathrm{Nd}_{2} \mathrm{O}_{3}$ & 0.000193 & 0.000780 & 0.000175 & 0.000177 & 0.000175 & 0.000143 & 0.000151 & 0.000886 \\
\hline $\mathrm{NiO}$ & 0.002029 & 0.007807 & 0.001772 & 0.001695 & 0.001773 & 0.001468 & 0.001456 & 0.008784 \\
\hline $\mathrm{P}_{2} \mathrm{O}_{5}$ & 0.019322 & 0.018899 & 0.012381 & 0.003254 & 0.020220 & 0.002849 & 0.020081 & 0.022483 \\
\hline $\mathrm{Pr}_{6} \mathrm{O}_{11}$ & 0.000021 & 0.000071 & 0.000014 & 0.000015 & 0.000014 & 0.000013 & 0.000014 & 0.000080 \\
\hline $\mathrm{SO}_{3}$ & 0.001352 & 0.005372 & 0.001194 & 0.001210 & 0.001213 & 0.001028 & 0.000997 & 0.006147 \\
\hline $\mathrm{SiO}_{2}$ & 0.540541 & 0.485292 & 0.513999 & 0.471114 & 0.465438 & 0.513421 & 0.543214 & 0.471566 \\
\hline $\mathrm{Sm}_{2} \mathrm{O}_{3}$ & 0.000021 & 0.000104 & 0.000021 & 0.000021 & 0.000021 & 0.000020 & 0.000021 & 0.000128 \\
\hline SrO & 0.000070 & 0.000352 & 0.000071 & 0.000072 & 0.000071 & 0.000066 & 0.000070 & 0.000432 \\
\hline $\mathrm{ThO}_{2}$ & 0.014405 & 0.013804 & 0.011529 & 0.010467 & 0.004989 & 0.005608 & 0.005357 & 0.005817 \\
\hline $\mathrm{TiO}_{2}$ & 0.005059 & 0.019707 & 0.004417 & 0.004382 & 0.004421 & 0.003689 & 0.003813 & 0.022304 \\
\hline $\mathrm{U}_{3} \mathrm{O}_{8}$ & 0.001773 & 0.000108 & 0.001743 & 0.001977 & 0.000091 & 0.000093 & 0.000107 & 0.001749 \\
\hline $\mathrm{Y}_{2} \mathrm{O}_{3}$ & 0.000032 & 0.000161 & 0.000033 & 0.000033 & 0.000033 & 0.000030 & 0.000032 & 0.000198 \\
\hline $\mathrm{ZnO}$ & 0.000177 & 0.000717 & 0.000181 & 0.000183 & 0.000181 & 0.000168 & 0.000178 & 0.000825 \\
\hline $\mathrm{ZrO}_{2}$ & 0.001581 & 0.006093 & 0.001373 & 0.001330 & 0.001374 & 0.001168 & 0.001177 & 0.006898 \\
\hline PCT B & 0.314 & 0.380 & 0.208 & 0.357 & 0.260 & 0.372 & 0.224 & 0.179 \\
\hline PCT Li & 0.299 & 0.355 & 0.257 & 0.358 & 0.265 & 0.317 & 0.233 & 0.206 \\
\hline PCT Na & 0.369 & 0.364 & 0.138 & 0.331 & 0.300 & 0.299 & 0.156 & 0.129 \\
\hline
\end{tabular}

* The glass numbers are those used in the reference from which the data were taken. 
Table A.9. WVDP Glass Compositions (mole fractions) and PCT Normalized B, Na, and Li Releases $\left(\mathrm{g} / \mathrm{m}^{2}\right)$

\begin{tabular}{|c|c|c|c|c|c|c|c|c|}
\hline Comp. & $\begin{array}{c}1^{*} \\
\text { WVDG-40 }\end{array}$ & $\begin{array}{c}2 \\
\text { WVDG-46 }\end{array}$ & $\begin{array}{c}3 \\
\text { FY92\#5 }\end{array}$ & $\begin{array}{c}4 \\
\text { FY92\#6 }\end{array}$ & $\begin{array}{c}5 \\
\text { FY92\#7 }\end{array}$ & $\begin{array}{c}6 \\
\text { FY92\#9 }\end{array}$ & $\begin{array}{c}7 \\
\text { FY92\#10 }\end{array}$ & $\begin{array}{c}8 \\
\text { FY92REF5 }\end{array}$ \\
\hline & 0.535544 & 0.513421 & 0.436609 & 0.526622 & 0.543849 & 0.516604 & 0.491458 & 0.491773 \\
\hline & 0.043326 & 0.047064 & 0.049811 & 0.033538 & 0.034635 & 0.033504 & 0.050969 & 0.045412 \\
\hline $\mathrm{ZrO}_{2}$ & 0.001354 & 0.001168 & 0.007620 & 0.007538 & 0.007784 & 0.007530 & 0.007797 & 0.001864 \\
\hline & 0.011511 & 0.005608 & 0.007193 & 0.007115 & 0.007348 & 0.011846 & 0.012267 & 0.009679 \\
\hline $\mathrm{Na}_{2} \mathrm{O}$ & 0.113291 & 0.113924 & 0.100421 & 0.116704 & 0.067061 & 0.064870 & 0.067173 & 0.113741 \\
\hline $\mathrm{K}_{2} \mathrm{O}$ & 0.024411 & 0.024526 & 0.051954 & 0.051392 & 0.053073 & 0.023356 & 0.053162 & 0.024235 \\
\hline $\mathrm{Li}_{2} \mathrm{O}$ & 0.065300 & 0.065613 & 0.121404 & 0.054629 & 0.056416 & 0.119966 & 0.056511 & 0.065106 \\
\hline $\mathrm{B}_{2} \mathrm{O}_{3}$ & 0.078458 & 0.120428 & 0.106670 & 0.105515 & 0.108967 & 0.105407 & 0.160378 & 0.132917 \\
\hline & 0.069725 & 0.044602 & 0.063696 & 0.042915 & 0.065068 & 0.062942 & 0.044393 & 0.054035 \\
\hline & 0.000473 & 0.000447 & 0.001206 & 0.001193 & 0.001232 & 0.001192 & 0.001234 & 0.000749 \\
\hline $\mathrm{CaO}$ & 0.009313 & 0.008312 & 0.006088 & 0.006022 & 0.006219 & 0.006016 & 0.006230 & 0.008705 \\
\hline $\mathrm{Ce}_{2} \mathrm{O}_{3}$ & 0.000885 & 0.000737 & 0.000672 & 0.000665 & 0.000686 & 0.000664 & 0.000687 & 0.000334 \\
\hline $\mathrm{CoO}$ & 0.000000 & 0.000000 & 0.000000 & 0.000000 & 0.000000 & 0.000000 & 0.000000 & 0.000192 \\
\hline $\mathrm{Cr}_{2} \mathrm{O}_{3}$ & 0.000382 & 0.000316 & 0.000655 & 0.000648 & 0.000669 & 0.000647 & 0.000670 & 0.000661 \\
\hline $\mathrm{Cs}_{2} \mathrm{O}$ & 0.000103 & 0.000097 & 0.000000 & 0.000000 & 0.000000 & 0.000000 & 0.000000 & 0.000204 \\
\hline $\mathrm{CuO}$ & 0.000182 & 0.000172 & 0.000000 & 0.000000 & 0.000000 & 0.000000 & 0.000000 & 0.000271 \\
\hline $\mathrm{La}_{2} \mathrm{O}_{3}$ & 0.000045 & 0.000042 & 0.000000 & 0.000000 & 0.000000 & 0.000000 & 0.000000 & 0.000088 \\
\hline $\mathrm{MgO}$ & 0.032036 & 0.028404 & 0.015707 & 0.015537 & 0.016046 & 0.015521 & 0.016072 & 0.015852 \\
\hline MnO & 0.001001 & 0.015455 & 0.008222 & 0.008133 & 0.008400 & 0.008125 & 0.008414 & 0.008340 \\
\hline $\mathrm{MoO}_{3}$ & 0.000101 & 0.000095 & 0.000000 & 0.000000 & 0.000000 & 0.000000 & 0.000000 & 0.000199 \\
\hline $\mathrm{Nd}_{2} \mathrm{O}_{3}$ & 0.000172 & 0.000143 & 0.000000 & 0.000000 & 0.000000 & 0.000000 & 0.000000 & 0.000299 \\
\hline งin & 0.001748 & 0.001468 & 0.002381 & 0.002355 & 0.002432 & 0.002352 & 0.002436 & 0.002403 \\
\hline & 0.003066 & 0.002849 & 0.006013 & 0.005948 & 0.006143 & 0.005942 & 0.006153 & 0.011986 \\
\hline & 0.000000 & 0.000000 & 0.000000 & 0.000000 & 0.000000 & 0.000000 & 0.000000 & 0.000176 \\
\hline $\mathrm{Pr}_{6} \mathrm{O}_{u}$ & 0.000014 & 0.000013 & 0.000000 & 0.000000 & 0.000000 & 0.000000 & 0.000000 & 0.000028 \\
\hline $\mathrm{RhO}_{2}$ & 0.000000 & 0.000000 & 0.000000 & 0.000000 & 0.000000 & 0.000000 & 0.000000 & 0.000106 \\
\hline & 0.000000 & 0.000000 & 0.000000 & 0.000000 & 0.000000 & 0.000000 & 0.000000 & 0.000432 \\
\hline $\mathrm{Sm}_{2} \overrightarrow{\mathrm{O}}_{3}$ & 0.000021 & 0.000020 & 0.000000 & 0.000000 & 0.000000 & 0.000000 & 0.000000 & 0.000062 \\
\hline $\mathrm{SO}_{3}$ & 0.001178 & 0.001028 & 0.002044 & 0.002021 & 0.002088 & 0.002019 & 0.002091 & 0.002062 \\
\hline Sro & 0.000070 & 0.000066 & 0.001716 & 0.001698 & 0.001753 & 0.001696 & 0.001756 & 0.000139 \\
\hline $\mathrm{TiO}_{2}$ & 0.004449 & 0.003689 & 0.007122 & 0.007045 & 0.007276 & 0.007038 & 0.007288 & 0.007188 \\
\hline $\mathrm{J}_{3} \mathrm{O}_{8}$ & 0.001630 & 0.000093 & 0.000522 & 0.000517 & 0.000533 & 0.000516 & 0.000534 & 0.000523 \\
\hline $\mathrm{Y}_{2} \mathrm{O}_{3}$ & 0.000032 & 0.000030 & 0.000000 & 0.000000 & 0.000000 & 0.000000 & 0.000000 & 0.000064 \\
\hline & 0.000178 & 0.000168 & 0.002273 & 0.002248 & 0.002322 & 0.002246 & 0.002325 & 0.000176 \\
\hline & 0.306 & 0.373 & 0.694 & 0.278 & 0.211 & 0.262 & 0.822 & 0.196 \\
\hline CT Na & & 0.300 & 0.529 & 0.267 & 0.208 & 0.196 & 0.569 & 0.256 \\
\hline CT $\mathrm{Li}$ & 0.305 & 0.318 & 0.584 & 0.357 & 0.264 & 0.295 & 0.711 & 0.275 \\
\hline
\end{tabular}

* The glass numbers are those used in the reference from which the data were taken. 
Table A.9. WVDP Glass Compositions (mole fractions) and PCT Normalized B, Na, and Li Releases $\left(\mathrm{g} / \mathrm{m}^{2}\right)$ (continued)

\begin{tabular}{|c|c|c|c|c|c|c|c|c|}
\hline Comp. & $\begin{array}{c}9 * \\
\text { Ratio2 }\end{array}$ & $\begin{array}{c}10 \\
\text { Ratio4 }\end{array}$ & $\begin{array}{c}11 \\
\text { Ratio5 }\end{array}$ & $\begin{array}{c}12 \\
\text { LoTh2 }\end{array}$ & $\begin{array}{c}13 \\
\text { LoTh4 }\end{array}$ & $\begin{array}{c}14 \\
\text { LoTh5 }\end{array}$ & $\begin{array}{r}15 \\
\mathrm{HiFe} 2\end{array}$ & $\begin{array}{r}16 \\
\text { HiFe3 }\end{array}$ \\
\hline $\mathrm{SiO}_{2}$ & 0.467398 & 0.507408 & 0.455155 & 0.493499 & 0.498396 & 0.494554 & 0.469548 & 0.460604 \\
\hline $\mathrm{Al}_{2} \mathrm{O}_{3}$ & 0.047106 & 0.039271 & 0.035947 & 0.042493 & 0.041431 & 0.038299 & 0.037635 & 0.038835 \\
\hline $\mathrm{ZrO}_{2}$ & 0.007013 & 0.010832 & 0.005720 & 0.007644 & 0.006766 & 0.006651 & 0.006648 & 0.006485 \\
\hline $\mathrm{ThO}_{2}$ & 0.003545 & 0.011440 & 0.007475 & 0.003699 & 0.004737 & 0.006477 & 0.008274 & 0.007836 \\
\hline $\mathrm{Na}_{2} \mathrm{O}$ & 0.102240 & 0.081605 & 0.102350 & 0.100185 & 0.099771 & 0.080481 & 0.102030 & 0.105904 \\
\hline $\mathrm{K}_{2} \mathrm{O}$ & 0.042045 & 0.041762 & 0.037413 & 0.033330 & 0.035405 & 0.033059 & 0.041196 & 0.043552 \\
\hline $\mathrm{Li}_{2} \mathrm{O}$ & 0.089163 & 0.096386 & 0.096710 & 0.091526 & 0.076731 & 0.077503 & 0.096194 & 0.101236 \\
\hline $\mathrm{B}_{2} \mathrm{O}_{3}$ & 0.113777 & 0.113012 & 0.147815 & 0.117252 & 0.137726 & 0.146677 & 0.113543 & 0.115087 \\
\hline $\mathrm{Fe}_{2} \mathrm{O}_{3}$ & 0.072418 & 0.044341 & 0.057290 & 0.056796 & 0.045684 & 0.061579 & 0.069749 & 0.065675 \\
\hline $\mathrm{BaO}$ & 0.001221 & 0.001191 & 0.001195 & 0.001183 & 0.001178 & 0.001208 & 0.001219 & 0.001210 \\
\hline $\mathrm{CaO}$ & 0.006163 & 0.006013 & 0.006033 & 0.005972 & 0.005947 & 0.006099 & 0.006151 & 0.006107 \\
\hline $\mathrm{Ce}_{2} \mathrm{O}_{3}$ & 0.000680 & 0.000663 & 0.000666 & 0.000659 & 0.000656 & 0.000673 & 0.000679 & 0.000674 \\
\hline $\mathrm{CoO}$ & 0.000000 & 0.000000 & 0.000000 & 0.000000 & 0.000000 & 0.000000 & 0.000000 & 0.000000 \\
\hline $\mathrm{Cr}_{2} \mathrm{O}_{3}$ & 0.000663 & 0.000647 & 0.000649 & 0.000643 & 0.000640 & 0.000656 & 0.000662 & 0.000657 \\
\hline $\mathrm{Cs}_{2} \mathrm{O}$ & 0.000000 & 0.000000 & 0.000000 & 0.000000 & 0.000000 & 0.000000 & 0.000000 & 0.000000 \\
\hline $\mathrm{CuO}$ & 0.000000 & 0.000000 & 0.000000 & 0.000000 & 0.000000 & 0.000000 & 0.000000 & 0.000000 \\
\hline $\mathrm{La}_{2} \mathrm{O}_{3}$ & 0.000000 & 0.000000 & 0.000000 & 0.000000 & 0.000000 & 0.000000 & 0.000000 & 0.000000 \\
\hline $\mathrm{MgO}$ & 0.015901 & 0.015512 & 0.015564 & 0.015406 & 0.015342 & 0.015735 & 0.015868 & 0.015755 \\
\hline MnO & 0.008324 & 0.008120 & 0.008148 & 0.008065 & 0.008031 & 0.008237 & 0.008307 & 0.008247 \\
\hline $\mathrm{MoO}_{3}$ & 0.000000 & 0.000000 & 0.000000 & 0.000000 & 0.000000 & 0.000000 & 0.000000 & 0.000000 \\
\hline $\mathrm{Nd}_{2} \mathrm{O}_{3}$ & 0.000000 & 0.000000 & 0.000000 & 0.000000 & 0.000000 & 0.000000 & 0.000000 & 0.000000 \\
\hline $\mathrm{NiO}$ & 0.002410 & 0.002351 & 0.002359 & 0.002335 & 0.002325 & 0.002385 & 0.002405 & 0.002388 \\
\hline $\mathrm{P}_{2} \mathrm{O}_{5}$ & 0.006088 & 0.005939 & 0.005959 & 0.005898 & 0.005874 & 0.006024 & 0.006075 & 0.006032 \\
\hline PdO & 0.000000 & 0.000000 & 0.000000 & 0.000000 & 0.000000 & 0.000000 & 0.000000 & 0.000000 \\
\hline $\mathrm{Pr}_{6} \mathrm{O}_{11}$ & 0.000000 & 0.000000 & 0.000000 & 0.000000 & 0.000000 & 0.000000 & 0.000000 & 0.000000 \\
\hline $\mathrm{RhO}_{2}$ & 0.000000 & 0.000000 & 0.000000 & 0.000000 & 0.000000 & 0.000000 & 0.000000 & 0.000000 \\
\hline $\mathrm{RuO}_{2}$ & 0.000000 & 0.000000 & 0.000000 & 0.000000 & 0.000000 & 0.000000 & 0.000000 & 0.000000 \\
\hline $\mathrm{Sm}_{2} \mathrm{O}_{3}$ & 0.000000 & 0.000000 & 0.000000 & 0.000000 & 0.000000 & 0.000000 & 0.000000 & 0.000000 \\
\hline $\mathrm{SO}_{3}$ & 0.002069 & 0.002018 & 0.002025 & 0.002004 & 0.001996 & 0.002047 & 0.002064 & 0.002050 \\
\hline Sro & 0.001737 & 0.001695 & 0.001701 & 0.001683 & 0.001676 & 0.001719 & 0.001734 & 0.001721 \\
\hline $\mathrm{TiO}_{2}$ & 0.007210 & 0.007034 & 0.007057 & 0.006986 & 0.006957 & 0.007135 & 0.007195 & 0.007144 \\
\hline $\mathrm{U}_{3} \mathrm{O}_{8}$ & 0.000529 & 0.000516 & 0.000517 & 0.000512 & 0.000510 & 0.000523 & 0.000528 & 0.000524 \\
\hline $\mathrm{Y}_{2} \mathrm{O}_{3}$ & 0.000000 & 0.000000 & 0.000000 & 0.000000 & 0.000000 & 0.000000 & 0.000000 & 0.000000 \\
\hline $\mathrm{ZnO}$ & 0.002301 & 0.002244 & 0.002252 & 0.002229 & 0.002220 & 0.002277 & 0.002296 & 0.002279 \\
\hline РСТ B & 1.546 & 0.261 & 0.244 & 0.393 & 0.644 & 0.242 & 0.588 & 0.418 \\
\hline PCT Na & 0.909 & 0.255 & 0.297 & 0.229 & 0.487 & 0.385 & 0.498 & 0.382 \\
\hline PCT Li & 1.129 & 0.290 & 0.305 & 0.283 & 0.577 & 0.433 & 0.555 & 0.423 \\
\hline
\end{tabular}

* The glass numbers are those used in the reference from which the data were taken. 
Table A.9. WVDP Glass Compositions (mole fractions) and PCT Normalized B, $\mathrm{Na}$, and $\mathrm{Li}$ Releases $\left(\mathrm{g} / \mathrm{m}^{2}\right)$ (continued)

\begin{tabular}{|c|c|c|c|c|c|c|c|c|}
\hline Comp. & $\begin{array}{c}17 * \\
\text { HiFe4 }\end{array}$ & $\begin{array}{c}18 \\
\text { PNL190 }\end{array}$ & $\begin{array}{c}19 \\
\text { Alkali1 }\end{array}$ & $\begin{array}{c}20 \\
\text { Alkali2 }\end{array}$ & $\begin{array}{c}21 \\
\text { Alkali3 }\end{array}$ & $\begin{array}{c}22 \\
\text { Alkali4 }\end{array}$ & $\begin{array}{c}23 \\
\text { Alkali5 }\end{array}$ & $\begin{array}{c}24 \\
\text { Alkali6 }\end{array}$ \\
\hline $\mathrm{SiO}_{2}$ & 0.457673 & 0.465367 & 0.508815 & 0.460112 & 0.477124 & 0.512849 & 0.476404 & 0.480355 \\
\hline $\mathrm{Al}_{2} \mathrm{O}_{3}$ & 0.037883 & 0.033261 & 0.046069 & 0.041230 & 0.041893 & 0.044116 & 0.043052 & 0.045291 \\
\hline $\mathrm{ZrO}_{2}$ & 0.005349 & 0.007302 & 0.008413 & 0.008327 & 0.008841 & 0.008796 & 0.007182 & 0.008089 \\
\hline $\mathrm{ThO}_{2}$ & 0.008590 & 0.011796 & 0.010831 & 0.010848 & 0.008957 & 0.009927 & 0.010324 & 0.009369 \\
\hline $\mathrm{Na}_{2} \mathrm{O}$ & 0.094911 & 0.115019 & 0.085363 & 0.087370 & 0.094820 & 0.089148 & 0.091386 & 0.089663 \\
\hline $\mathrm{K}_{2} \mathrm{O}$ & 0.032730 & 0.050551 & 0.035674 & 0.034795 & 0.036521 & 0.033841 & 0.034575 & 0.038824 \\
\hline $\mathrm{Li}_{2} \mathrm{O}$ & 0.098431 & 0.118126 & 0.086136 & 0.097763 & 0.081547 & 0.082971 & 0.086482 & 0.091188 \\
\hline $\mathrm{B}_{2} \mathrm{O}_{3}$ & 0.149651 & 0.103392 & 0.107832 & 0.149425 & 0.136918 & 0.112944 & 0.147461 & 0.121539 \\
\hline $\mathrm{Fe}_{2} \mathrm{O}_{3}$ & 0.060359 & 0.042040 & 0.055964 & 0.055415 & 0.058343 & 0.051012 & 0.048769 & 0.060619 \\
\hline $\mathrm{BaO}$ & 0.001202 & 0.001174 & 0.001212 & 0.001208 & 0.001215 & 0.001201 & 0.001201 & 0.001216 \\
\hline $\mathrm{CaO}$ & 0.006066 & 0.005924 & 0.006120 & 0.006099 & 0.006134 & 0.006063 & 0.006060 & 0.006138 \\
\hline $\mathrm{Ce}_{2} \mathrm{O}_{3}$ & 0.000669 & 0.000654 & 0.000675 & 0.000673 & 0.000677 & 0.000669 & 0.000669 & 0.000677 \\
\hline $\mathrm{CoO}$ & 0.000000 & 0.000000 & 0.000000 & 0.000000 & 0.000000 & 0.000000 & 0.000000 & 0.000000 \\
\hline $\mathrm{Cr}_{2} \mathrm{O}_{3}$ & 0.000653 & 0.000638 & 0.000659 & 0.000656 & 0.000660 & 0.000652 & 0.000652 & 0.000660 \\
\hline $\mathrm{Cs}_{2} \mathrm{O}$ & 0.000000 & 0.000000 & 0.000000 & 0.000000 & 0.000000 & 0.000000 & 0.000000 & 0.000000 \\
\hline $\mathrm{CuO}^{*}$ & 0.000000 & 0.000000 & 0.000000 & 0.000000 & 0.000000 & 0.000000 & 0.000000 & 0.000000 \\
\hline $\mathrm{La}_{2} \mathrm{O}_{3}$. & 0.000000 & 0.000000 & 0.000000 & 0.000000 & 0.000000 & 0.000000 & 0.000000 & 0.000000 \\
\hline $\mathrm{MgO}$ & 0.015650 & 0.015283 & 0.015788 & 0.015734 & 0.015826 & 0.015642 & 0.015 .634 & 0.015834 \\
\hline $\mathrm{MnO}$ & 0.008193 & 0.008000 & 0.008265 & 0.008236 & 0.008285 & 0.008188 & 0.008184 & 0.008289 \\
\hline $\mathrm{MoO}_{3}$ & 0.000000 & 0.000000 & 0.000000 & 0.000000 & 0.000000 & 0.000000 & 0.000000 & 0.000000 \\
\hline $\mathrm{Nd}_{2} \mathrm{O}_{3}$ & 0.000000 & 0.000000 & 0.000000 & 0.000000 & 0.000000 & 0.000000 & 0.000000 & 0.000000 \\
\hline $\mathrm{NiO}$ & 0.002372 & 0.002316 & 0.002393 & 0.002385 & 0.002399 & 0.002371 & 0.002369 & 0.002400 \\
\hline $\mathrm{P}_{2} \mathrm{O}_{3}$ & 0.005992 & 0.005851 & 0.006044 & 0.006024 & 0.006059 & 0.005989 & 0.005985 & 0.006062 \\
\hline PdO & 0.000000 & 0.000000 & 0.000000 & 0.000000 & 0.000000 & 0.000000 & 0.000000 & 0.000000 \\
\hline $\mathrm{Pr}_{6} \mathrm{O}_{11}$ & 0.000000 & 0.000000 & 0.000000 & 0.000000 & 0.000000 & 0.000000 & 0.000000 & 0.000000 \\
\hline $\mathrm{RhO}_{2}$ & 0.000000 & 0.000000 & 0.000000 & 0.000000 & 0.000000 & 0.000000 & 0.000000 & 0.000000 \\
\hline $\mathrm{RuO}_{2}$ & 0.000000 & 0.000000 & 0.000000 & 0.000000 & 0.000000 & 0.000000 & 0.000000 & 0.000000 \\
\hline $\mathrm{Sm}_{2} \mathrm{O}_{3}$ & 0.000000 & 0.000000 & 0.000000 & 0.000000 & 0.000000 & 0.000000 & 0.000000 & 0.000000 \\
\hline $\mathrm{SO}_{3}$ & 0.002036 & 0.001988 & 0.002054 & 0.002047 & 0.002059 & 0.002035 & 0.002034 & 0.002060 \\
\hline SrO & 0.001710 & 0.001670 & 0.001725 & 0.001719 & 0.001729 & 0.001709 & 0.001708 & 0.001730 \\
\hline $\mathrm{TiO}_{2}$ & 0.007096 & 0.006930 & 0.007159 & 0.007134 & 0.007176 & 0.007093 & 0.007089 & 0.007180 \\
\hline $\mathrm{U}_{3} \mathrm{O}_{8}$ & 0.000520 & 0.000508 & 0.000525 & 0.000523 & 0.000526 & 0.000520 & 0.000520 & 0.000526 \\
\hline $\mathrm{Y}_{2} \mathrm{O}_{3}$ & 0.000000 & 0.000000 & 0.000000 & 0.000000 & 0.000000 & 0.000000 & 0.000000 & 0.000000 \\
\hline $\mathrm{ZnO}$ & 0.002264 & 0.002211 & 0.002284 & 0.002276 & 0.002290 & 0.002263 & 0.002262 & 0.002291 \\
\hline РСТ B & 0.741 & 1.227 & 0.251 & 0.647 & 0.454 & 0.274 & 0.636 & 0.328 \\
\hline PCT Na & 0.572 & 1.014 & 0.217 & 0.430 & 0.316 & 0.195 & 0.421 & 0.240 \\
\hline PCT Li & 0.652 & 1.069 & 0.280 & 0.474 & 0.355 & 0.257 & 0.477 & 0.302 \\
\hline
\end{tabular}

* The glass numbers are those used in the reference from which the data were taken. 
Table A.9. WVDP Glass Compositions (mole fractions) and PCT Normalized $\mathrm{B}, \mathrm{Na}$, and $\mathrm{Li}$ Releases $\left(\mathrm{g} / \mathrm{m}^{2}\right)$ (continued)

\begin{tabular}{|c|c|c|c|c|c|c|c|c|}
\hline Comp. & $\begin{array}{r}25^{*} \\
\text { Alkali7 }\end{array}$ & $\begin{array}{c}26 \\
\text { Alkali8 }\end{array}$ & $\begin{array}{c}27 \\
\text { Alkali9 }\end{array}$ & $\begin{array}{c}28 \\
\text { Ref6Q2 }\end{array}$ & $\begin{array}{c}29 \\
\text { FY93\#1 }\end{array}$ & $\begin{array}{c}30 \\
\text { FY93\#2 }\end{array}$ & $\begin{array}{c}31 \\
\text { FY93\#3 }\end{array}$ & $\begin{array}{c}32 \\
\text { FY93 } \# 4\end{array}$ \\
\hline $\mathrm{SiO}_{2}$ & 0.461738 & 0.483394 & 0.499984 & 0.484195 & 0.481539 & 0.481008 & 0.402515 & 0.559838 \\
\hline $\mathrm{Al}_{2} \mathrm{O}_{3}$ & 0.040465 & 0.039194 & 0.046624 & 0.041776 & 0.055402 & 0.029330 & 0.051866 & 0.030436 \\
\hline $\mathrm{ZrO}_{2}$ & 0.009237 & 0.008340 & 0.009370 & 0.007605 & 0.007849 & 0.007468 & 0.007348 & 0.007749 \\
\hline $\mathrm{ThO}_{2}$ & 0.011127 & 0.010649 & 0.011310 & 0.009572 & 0.004939 & 0.004699 & 0.004624 & 0.004877 \\
\hline $\mathrm{Na}_{2} \mathrm{O}$ & 0.093542 & 0.103625 & 0.082796 & 0.091634 & 0.067617 & 0.115620 & 0.113765 & 0.066761 \\
\hline $\mathrm{K}_{2} \mathrm{O}$ & 0.040781 & 0.041668 & 0.038588 & 0.037683 & 0.024345 & 0.050914 & 0.050098 & 0.052836 \\
\hline $\mathrm{Li}_{2} \mathrm{O}$ & 0.095225 & 0.078810 & 0.081096 & 0.088142 & 0.056884 & 0.054122 & 0.117066 & 0.056164 \\
\hline $\mathrm{B}_{2} \mathrm{O}_{3}$ & 0.142031 & 0.128131 & 0.127970 & 0.131444 & 0.174278 & 0.165814 & 0.163154 & 0.095803 \\
\hline $\mathrm{Fe}_{2} \mathrm{O}_{3}$ & 0.051228 & 0.051390 & 0.047532 & 0.053436 & 0.070884 & 0.037497 & 0.036895 & 0.069987 \\
\hline $\mathrm{BaO}$ & 0.001206 & 0.001210 & 0.001209 & 0.001204 & 0.001242 & 0.001182 & 0.001163 & 0.001227 \\
\hline $\mathrm{CaO}$ & 0.006089 & 0.006108 & 0.006100 & 0.006076 & 0.006271 & 0.005967 & 0.005871 & 0.006192 \\
\hline $\mathrm{Ce}_{2} \mathrm{O}_{3}$ & 0.000672 & 0.000674 & 0.000673 & 0.000670 & 0.000692 & 0.000658 & 0.000648 & 0.000683 \\
\hline $\mathrm{CoO}$ & 0.000000 & 0.000000 & 0.000000 & 0.000000 & 0.000000 & 0.000000 & 0.000000 & 0.000000 \\
\hline $\mathrm{Cr}_{2} \mathrm{O}_{3}$ & 0.000655 & 0.000657 & 0.000656 & 0.000654 & 0.000675 & 0.000642 & 0.000632 & 0.000666 \\
\hline $\mathrm{Cs}_{2} \mathrm{O}$ & 0.000000 & 0.000000 & 0.000000 & 0.000000 & 0.000000 & 0.000000 & 0.000000 & 0.000000 \\
\hline CuO & 0.000000 & 0.000000 & 0.000000 & 0.000000 & 0.000000 & 0.000000 & 0.000000 & 0.000000 \\
\hline $\mathrm{La}_{2} \mathrm{O}_{3}$ & 0.000000 & 0.000000 & 0.000000 & 0.000000 & 0.000000 & 0.000000 & 0.000000 & 0.000000 \\
\hline $\mathrm{MgO}$ & 0.015708 & 0.015758 & 0.015738 & 0.015676 & 0.016179 & 0.015393 & 0.015146 & 0.015974 \\
\hline MnO & 0.008223 & 0.008249 & 0.008239 & 0.008206 & 0.008469 & 0.008058 & 0.007929 & 0.008362 \\
\hline $\mathrm{MoO}_{3}$ & 0.000000 & 0.000000 & 0.000000 & 0.000000 & 0.000000 & 0.000000 & 0.000000 & 0.000000 \\
\hline $\mathrm{Nd}_{2} \mathrm{O}_{3}$ & 0.000000 & 0.000000 & 0.000000 & 0.000000 & 0.000000 & 0.000000 & 0.000000 & 0.000000 \\
\hline $\mathrm{NiO}$ & 0.002381 & 0.002388 & 0.002385 & 0.002376 & 0.002452 & 0.002333 & 0.002296 & 0.002421 \\
\hline $\mathrm{P}_{2} \mathrm{O}_{5}$ & 0.006014 & 0.006033 & 0.006025 & 0.006002 & 0.006194 & 0.005893 & 0.005799 & 0.006116 \\
\hline PdO & 0.000000 & 0.000000 & 0.000000 & 0.000000 & 0.000000 & 0.000000 & 0.000000 & 0.000000 \\
\hline $\mathrm{Pr}_{6} \mathrm{O}_{11}$ & 0.000000 & 0.000000 & 0.000000 & 0.000000 & 0.000000 & 0.000000 & 0.000000 & 0.000000 \\
\hline $\mathrm{RhO}_{2}$ & 0.000000 & 0.000000 & 0.000000 & 0.000000 & 0.000000 & 0.000000 & 0.000000 & 0.000000 \\
\hline $\mathrm{RuO}_{2}$ & 0.000000 & 0.000000 & 0.000000 & 0.000000 & 0.000000 & 0.000000 & 0.000000 & 0.000000 \\
\hline $\mathrm{Sm}_{2} \mathrm{O}_{3}$ & 0.000000 & 0.000000 & 0.000000 & 0.000000 & 0.000000 & 0.000000 & 0.000000 & 0.000000 \\
\hline $\mathrm{SO}_{3}$ & 0.002044 & 0.002050 & 0.002048 & 0.002040 & 0.002105 & 0.002003 & 0.001971 & 0.002078 \\
\hline Sro & 0.001716 & 0.001722 & 0.001720 & 0.001713 & 0.001768 & 0.001682 & 0.001655 & 0.001745 \\
\hline $\mathrm{TiO}_{2}$ & 0.007123 & 0.007145 & 0.007136 & 0.007108 & 0.007336 & 0.006980 & 0.006868 & 0.007243 \\
\hline $\mathrm{U}_{3} \mathrm{O}_{8}$ & 0.000522 & 0.000524 & 0.000523 & 0.000521 & 0.000538 & 0.000512 & 0.000504 & 0.000531 \\
\hline $\mathrm{Y}_{2} \mathrm{O}_{3}$ & 0.000000 & 0.000000 & 0.000000 & 0.000000 & 0.000000 & 0.000000 & 0.000000 & 0.000000 \\
\hline $\mathrm{ZnO}$ & 0.002273 & 0.002280 & 0.002277 & 0.002268 & 0.002341 & 0.002227 & 0.002191 & 0.002311 \\
\hline РСТ В & 0.889 & 0.468 & 0.284 & 0.415 & 0.544 & 4.044 & 3.266 & 0.239 \\
\hline PCT Na & 0.567 & 0.350 & 0.208 & 0.317 & 0.328 & 2.426 & 1.897 & 0.190 \\
\hline PCT Li & 0.586 & 0.412 & 0.271 & 0.353 & 0.504 & 2.702 & 2.265 & 0.250 \\
\hline
\end{tabular}

* The glass numbers are those used in the reference from which the data were taken. 
Table A.9. WVDP Glass Compositions (mole fractions) and PCT Normalized B, Na, and Li Releases $\left(\mathrm{g} / \mathrm{m}^{2}\right)$ (continued)

\begin{tabular}{|c|c|c|c|c|c|c|c|c|}
\hline Comp. & $\begin{array}{c}33^{*} \\
\text { FY93\#5 }\end{array}$ & $\begin{array}{c}34 \\
\text { FY93\#6 }\end{array}$ & $\begin{array}{c}35 \\
\text { FY93\$7 }\end{array}$ & $\begin{array}{c}36 \\
\text { FY93\#8 }\end{array}$ & $\begin{array}{c}37 \\
\text { FY93\#9 }\end{array}$ & $\begin{array}{c}8 \\
\text { FY93\#10 }\end{array}$ & $\begin{array}{c}39 \\
\text { FY94\#1 }\end{array}$ & $\begin{array}{c}40 \\
\text { FY94 } \$ 2\end{array}$ \\
\hline $\mathrm{SiO}_{2}$ & 0.519219 & 0.528517 & 0.557559 & 0.432517 & 0.441461 & 0.521456 & 0.555001 & 0.521361 \\
\hline $\mathrm{Al}_{2} \mathrm{O}_{3}$ & 0.027934 & 0.050950 & 0.054619 & 0.031393 & 0.057586 & 0.028528 & 0.050695 & 0.052872 \\
\hline $\mathrm{ZrO}_{2}$ & 0.007112 & 0.007218 & 0.007738 & 0.007993 & 0.008158 & 0.007263 & 0.004138 & 0.004316 \\
\hline $\mathrm{ThO}_{2}$ & 0.004476 & 0.004542 & 0.014608 & 0.015090 & 0.015402 & 0.013713 & 0.012698 & 0.007532 \\
\hline $\mathrm{Na}_{2} \mathrm{O}$ & 0.061271 & 0.111756 & 0.066661 & 0.123754 & 0.126313 & 0.112458 & 0.094669 & 0.061121 \\
\hline $\mathrm{K}_{2} \mathrm{O}$ & 0.022061 & 0.022389 & 0.052757 & 0.024793 & 0.055623 & 0.022530 & 0.044493 & 0.045630 \\
\hline $\mathrm{Li}_{2} \mathrm{O}$ & 0.113312 & 0.097410 & 0.056080 & 0.057929 & 0.062440 & 0.115721 & 0.065453 & 0.097519 \\
\hline $\mathrm{B}_{2} \mathrm{O}_{3}$ & 0.157922 & 0.089234 & 0.095660 & 0.177051 & 0.100857 & 0.089795 & 0.090301 & 0.074192 \\
\hline $\mathrm{Fe}_{2} \mathrm{O}_{3}$ & 0.035712 & 0.036244 & 0.038854 & 0.072186 & 0.073679 & 0.036471 & 0.028913 & 0.079514 \\
\hline $\mathrm{BaO}$ & 0.001126 & 0.001143 & 0.001225 & 0.001265 & 0.001291 & 0.001150 & 0.001184 & 0.001235 \\
\hline $\mathrm{CaO}$ & 0.005683 & 0.005767 & 0.006182 & 0.006386 & 0.006518 & 0.005803 & 0.005979 & 0.006235 \\
\hline $\mathrm{Ce}_{2} \mathrm{O}_{3}$ & 0.000627 & 0.000636 & 0.000682 & 0.000705 & 0.000719 & 0.000640 & 0.000660 & 0.000688 \\
\hline $\mathrm{CoO}$ & 0.000000 & 0.000000 & 0.000000 & 0.000000 & 0.000000 & 0.000000 & 0.000000 & 0.000000 \\
\hline $\mathrm{Cr}_{2} \mathrm{O}_{3}$ & 0.000612 & 0.000621 & 0.000665 & 0.000687 & 0.000701 & 0.000625 & 0.000643 & 0.000671 \\
\hline $\mathrm{Cs}_{2} \mathrm{O}$ & $0: 000000$ & 0.000000 & 0.000000 & 0.000000 & 0.000000 & 0.000000 & 0.000000 & 0.000000 \\
\hline CuO & 0.000000 & 0.000000 & 0.000000 & 0.000000 & 0.000000 & 0.000000 & 0.000000 & 0.000000 \\
\hline $\mathrm{La}_{2} \mathrm{O}_{3}$ & 0.000000 & 0.000000 & 0.000000 & 0.000000 & 0.000000 & 0.000000 & 0.000000 & 0.000000 \\
\hline $\mathrm{MgO}^{\circ}$ & 0.014660 & 0.014879 & 0.015950 & 0.016476 & 0.016817 & 0.014972 & 0.015424 & 0.016087 \\
\hline $\mathrm{MnO}$ & 0.007674 & 0.007789 & 0.008349 & 0.008625 & 0.008803 & 0.007838 & 0.008074 & 0.008421 \\
\hline $\mathrm{MoO}_{3}$ & 0.000000 & 0.000000 & 0.000000 & 0.000000 & 0.000000 & 0.000000 & 0.000000 & 0.000000 \\
\hline $\mathrm{Nd}_{2} \mathrm{O}_{3}$ & 0.000000 & 0.000000 & 0.000000 & 0.000000 & 0.000000 & 0.000000 & 0.000000 & 0.000000 \\
\hline $\mathrm{NiO}$ & 0.002222 & 0.002255 & 0.002417 & 0.002497 & 0.002549 & 0.002269 & 0.002338 & 0.002438 \\
\hline $\mathrm{P}_{2} \mathrm{O}_{3}$ & 0.005613 & 0.005696 & 0.006106 & 0.006308 & 0.006438 & 0.005732 & 0.005905 & 0.006159 \\
\hline PdO & 0.000000 & 0.000000 & 0.000000 & 0.000000 & 0.000000 & 0.000000 & 0.000000 & 0.000000 \\
\hline $\mathrm{Pr}_{6} \mathrm{O}_{11}$ & 0.000000 & 0.000000 & 0.000000 & 0.000000 & 0.000000 & 0.000000 & 0.000000 & 0.000000 \\
\hline $\mathrm{RhO}_{2}$ & 0.000000 & 0.000000 & 0.000000 & 0.000000 & 0.000000 & 0.000000 & 0.000000 & 0.000000 \\
\hline $\mathrm{RuO}_{2}$ & 0.000000 & 0.000000 & 0.000000 & 0.000000 & 0.000000 & 0.000000 & 0.000000 & 0.000000 \\
\hline $\mathrm{Sm}_{2} \mathrm{O}_{3}$ & 0.000000 & 0.000000 & 0.000000 & 0.000000 & 0.000000 & 0.000000 & 0.000000 & 0.000000 \\
\hline $\mathrm{SO}_{3}$ & 0.001907 & 0.001936 & 0.002075 & 0.002144 & 0.002188 & 0.001948 & 0.002007 & 0.002093 \\
\hline Sro & 0.001602 & 0.001626 & 0.001743 & 0.001800 & 0.001837 & 0.001636 & 0.001685 & 0.001758 \\
\hline $\mathrm{TiO}_{2}$ & 0.006647 & 0.006746 & 0.007232 & 0.007471 & 0.007625 & 0.006789 & 0.006994 & 0.007294 \\
\hline $\mathrm{U}_{3} \mathrm{O}_{8}$ & 0.000487 & 0.000495 & 0.000530 & 0.000548 & 0.000559 & 0.000498 & 0.000513 & 0.000535 \\
\hline $\mathrm{Y}_{2} \mathrm{O}_{3}$ & 0.000000 & 0.000000 & 0.000000 & 0.000000 & 0.000000 & 0.000000 & 0.000000 & 0.000000 \\
\hline $\mathrm{ZnO}$ & 0.002121 & 0.002153 & 0.002308 & 0.002384 & 0.002433 & 0.002166 & 0.002232 & 0.002328 \\
\hline РСТ B & 1.267 & 0.269 & 0.186 & 1.575 & 0.431 & 0.757 & 0.186 & 0.215 \\
\hline PCT Na & 0.658 & 0.225 & 0.145 & 0.962 & 0.377 & 0.467 & 0.208 & 0.222 \\
\hline PCT Li & 0.881 & 0.266 & 0.200 & 1.197 & 0.385 & 0.495 & 0.253 & 0.297 \\
\hline
\end{tabular}

* The glass numbers are those used in the reference from which the data were taken. 
Table A.9. WVDP Glass Compositions (mole fractions) and PCT Normalized B, Na, and $\mathrm{Li}$ Releases $\left(\mathrm{g} / \mathrm{m}^{2}\right)$ (continued)

\begin{tabular}{|c|c|c|c|c|c|c|c|c|}
\hline Comp. & $\begin{array}{c}41^{*} \\
\text { FY94\#3 }\end{array}$ & $\begin{array}{c}42 \\
\text { FY } 94 \# 4\end{array}$ & $\begin{array}{c}43 \\
\text { FY94\#5 }\end{array}$ & $\begin{array}{c}44 \\
\text { FY94\#6 }\end{array}$ & $\begin{array}{c}45 \\
\text { FY94\#7 }\end{array}$ & $\begin{array}{c}46 \\
\text { FY94 } \$ 88\end{array}$ & $\begin{array}{c}47 \\
\text { FY94 }\end{array}$ & $\begin{array}{c}48 \\
\text { FY94\#10 }\end{array}$ \\
\hline $\mathrm{SiO}_{2}$ & 0.433979 & 0.406858 & 0.534509 & 0.483946 & 0.510653 & 0.414041 & 0.492486 & 0.470732 \\
\hline $\mathrm{Al}_{2} \mathrm{O}_{3}$ & 0.039121 & 0.029883 & 0.036318 & 0.055976 & 0.043582 & 0.031117 & 0.032356 & 0.040420 \\
\hline $\mathrm{ZrO}_{2}$ & 0.004175 & 0.004198 & 0.009453 & 0.011475 & 0.010533 & 0.011177 & 0.010477 & 0.003990 \\
\hline $\mathrm{ThO}_{2}$ & 0.005338 & 0.002415 & 0.007905 & 0.014467 & 0.012555 . & 0.003004 & 0.006519 & 0.009182 \\
\hline $\mathrm{Na}_{2} \mathrm{O}$ & 0.125077 & 0.104036 & 0.116235 & 0.071663 & 0.075110 & 0.099357 & 0.070816 & 0.084749 \\
\hline $\mathrm{K}_{2} \mathrm{O}$ & 0.034415 & 0.039868 & 0.047175 & 0.033007 & 0.029952 & 0.029089 & 0.048727 & 0.022377 \\
\hline $\mathrm{Li}_{2} \mathrm{O}$ & 0.066037 & 0.109079 & 0.100267 & 0.116435 & 0.056651 & 0.091700 & 0.081603 & 0.108175 \\
\hline $\mathrm{B}_{2} \mathrm{O}_{3}$ & 0.189197 & 0.174043 & 0.068568 & 0.075389 & 0.177606 & 0.186438 & 0.139071 & 0.180789 \\
\hline $\mathrm{Fe}_{2} \mathrm{O}_{3}$ & 0.048544 & 0.075209 & 0.027869 & 0.080798 & 0.029195 & 0.078704 & 0.062874 & 0.027874 \\
\hline $\mathrm{BaO}$ & 0.001195 & 0.001202 & 0.001142 & 0.001255 & 0.001196 & 0.001223 & 0.001216 & 0.001142 \\
\hline $\mathrm{CaO}$ & 0.006032 & 0.006065 & 0.005763 & 0.006336 & 0.006037 & 0.006172 & 0.006139 & 0.005764 \\
\hline $\mathrm{Ce}_{2} \mathrm{O}_{3}$ & 0.000666 & 0.000669 & 0.000636 & 0.000699 & 0.000666 & 0.000681 & 0.000677 & 0.000636 \\
\hline $\mathrm{CoO}$ & 0.000000 & 0.000000 & 0.000000 & 0.000000 & 0.000000 & 0.000000 & 0.000000 & 0.000000 \\
\hline $\mathrm{C}_{T_{2}} \mathrm{O}_{3}$ & 0.000649 & 0.000653 & 0.000620 & 0.000682 & 0.000650 & 0.000664 & 0.000661 & 0.000620 \\
\hline $\mathrm{Cs}_{2} \mathrm{O}$ & 0.000000 & 0.000000 & 0.000000 & 0.000000 & 0.000000 & 0.000000 & 0.000000 & 0.000000 \\
\hline CuO & 0.000000 & 0.000000 & 0.000000 & 0.000000 & 0.000000 & 0.000000 & 0.000000 & 0.000000 \\
\hline $\mathrm{La}_{2} \mathrm{O}_{3}$ & 0.000000 & 0.000000 & 0.000000 & 0.000000 & 0.000000 & 0.000000 & 0.000000 & 0.000000 \\
\hline $\mathrm{MgO}$ & 0.015562 & 0.015647 & 0.014867 & 0.016346 & 0.015575 & 0.015923 & 0.015837 & 0.014870 \\
\hline MnO & 0.008146 & 0.008191 & 0.007783 & 0.008557 & 0.008153 & 0.008335 & 0.008290 & 0.007784 \\
\hline $\mathrm{MoO}_{3}$ & 0.000000 & 0.000000 & 0.000000 & 0.000000 & 0.000000 & 0.000000 & 0.000000 & 0.000000 \\
\hline $\mathrm{Nd}_{2} \mathrm{O}_{3}$ & 0.000000 & 0.000000 & 0.000000 & 0.000000 & 0.000000 & 0.000000 & 0.000000 & 0.000000 \\
\hline $\mathrm{NiO}$ & 0.002359 & 0.002371 & 0.002253 & 0.002477 & 0.002361 & 0.002413 & 0.002400 & 0.002254 \\
\hline $\mathrm{P}_{2} \mathrm{O}_{5}$ & 0.005958 & 0.005990 & 0.005692 & 0.006258 & 0.005963 & 0.006096 & 0.006063 & 0.005693 \\
\hline PdO & 0.000000 & 0.000000 & 0.000000 & 0.000000 & 0.000000 & 0.000000 & 0.000000 & 0.000000 \\
\hline $\mathrm{Pr}_{6} \mathrm{O}_{11}$ & 0.000000 & 0.000000 & 0.000000 & 0.000000 & 0.000000 & 0.000000 & 0.000000 & 0.000000 \\
\hline $\mathrm{RhO}_{2}$ & 0.000000 & 0.000000 & 0.000000 & 0.000000 & 0.000000 & 0.000000 & 0.000000 & 0.000000 \\
\hline $\mathrm{RuO}_{2}$ & 0.000000 & 0.000000 & 0.000000 & 0.000000 & 0.000000 & 0.000000 & 0.000000 & 0.000000 \\
\hline $\mathrm{Sm}_{2} \mathrm{O}_{3}$ & 0.000000 & 0.000000 & 0.000000 & 0.000000 & 0.000000 & 0.000000 & 0.000000 & 0.000000 \\
\hline $\mathrm{SO}_{3}$ & 0.002025 & 0.002036 & 0.001934 & 0.002127 & 0.002026 & 0.002072 & 0.002060 & 0.001935 \\
\hline SrO & 0.001700 & 0.001710 & 0.001624 & 0.001786 & 0.001702 & 0.001740 & 0.001730 & 0.001625 \\
\hline $\mathrm{TiO}_{2}$ & 0.007056 & 0.007095 & 0.006741 & 0.007412 & 0.007062 & 0.007220 & 0.007181 & 0.006743 \\
\hline $\mathrm{U}_{3} \mathrm{O}_{8}$ & 0.000517 & 0.000520 & 0.000494 & 0.000543 & 0.000518 & 0.000529 & 0.000527 & 0.000494 \\
\hline $\mathrm{Y}_{2} \mathrm{O}_{3}$ & 0.000000 & 0.000000 & 0.000000 & 0.000000 & 0.000000 & 0.000000 & 0.000000 & 0.000000 \\
\hline $\mathrm{ZnO}$ & 0.002252 & 0.002264 & 0.002151 & 0.002365 & 0.002253 & 0.002304 & 0.002291 & 0.002152 \\
\hline РCT B & 4.057 & 3.336 & 0.453 & 0.288 & 1.047 & 2.242 & 0.435 & 3.385 \\
\hline PCT Na & 2.553 & 2.296 & 0.495 & 0.266 & 0.693 & 1.565 & 0.368 & 2.151 \\
\hline PCT Li & 3.559 & 2.605 & 0.532 & 0.364 & 0.949 & 1.920 & 0.447 & 2.885 \\
\hline
\end{tabular}

* The glass numbers are those used in the reference from which the data were taken. 
Table A.9. WVDP Glass Compositions (mole fractions) and PCT Normalized $\mathrm{B}, \mathrm{Na}$, and $\mathrm{Li}$ Releases $\left(\mathrm{g} / \mathrm{m}^{2}\right)$ (continued)

\begin{tabular}{|c|c|c|c|c|c|c|c|c|}
\hline Comp. & $\begin{array}{r}49 * \\
\text { Sigma1 }\end{array}$ & $\begin{array}{c}50 \\
\text { Sigma2 }\end{array}$ & $\begin{array}{c}51 \\
\text { Sigma3 }\end{array}$ & $\begin{array}{c}52 \\
\text { Sigma4 }\end{array}$ & $\begin{array}{c}53 \\
\text { Sigma5 }\end{array}$ & $\begin{array}{c}54 \\
\text { Sigma6 }\end{array}$ & $\begin{array}{c}55 \\
\text { Sigma7 }\end{array}$ & $\begin{array}{c}56 \\
\text { Sigma8 }\end{array}$ \\
\hline $\mathrm{SiO}_{2}$ & 0.389960 & 0.392802 & 0.441782 & 0.403975 & 0.400893 & 0.417742 & 0.410534 & 0.425531 \\
\hline $\mathrm{Al}_{2} \mathrm{O}_{3}$ & 0.028809 & 0.029019 & 0.032613 & 0.032858 & 0.031049 & 0.031699 & 0.034272 & 0.032531 \\
\hline $\mathrm{ZrO}_{2}$ & 0.004056 & 0.004086 & 0.004576 & 0.004495 & 0.004468 & 0.004576 & 0.004337 & 0.004711 \\
\hline $\mathrm{ThO}_{2}$ & 0.002308 & 0.013478 & 0.010513 & 0.002590 & 0.002346 & 0.005730 & 0.002855 & 0.002617 \\
\hline $\mathrm{Na}_{2} \mathrm{O}$ & 0.121520 & 0.122406 & 0.127130 & 0.113627 & 0.116592 & 0.112061 & 0.118290 & 0.108148 \\
\hline $\mathrm{K}_{2} \mathrm{O}$ & 0.050010 & 0.050374 & 0.051417 & 0.046455 & 0.048951 & 0.047452 & 0.048009 & 0.046217 \\
\hline $\mathrm{Li}_{2} \mathrm{O}$ & 0.116861 & 0.117713 & 0.118539 & 0.114408 & 0.112854 & 0.110463 & 0.113504 & 0.112158 \\
\hline $\mathrm{B}_{2} \mathrm{O}_{3}$ & 0.183818 & 0.185157 & 0.079018 & 0.181692 & 0.177939 & 0.172859 & 0.167314 & 0.158814 \\
\hline $\mathrm{Fe}_{2} \mathrm{O}_{3}$ & 0.050080 & 0.032003 & 0.078901 & 0.047397 & 0.052060 & 0.044612 & 0.048270 & 0.056210 \\
\hline $\mathrm{BaO}$ & 0.001161 & 0.001170 & 0.001226 & 0.001159 & 0.001167 & 0.001166 & 0.001162 & 0.001172 \\
\hline $\mathrm{CaO}$ & 0.005861 & 0.005903 & 0.006187 & 0.005852 & 0.005891 & 0.005886 & 0.005865 & 0.005915 \\
\hline $\mathrm{Ce}_{2} \mathrm{O}_{3}$ & 0.000647 & 0.000651 & 0.000683 & 0.000646 & 0.000650 & 0.000649 & 0.000647 & 0.000653 \\
\hline $\mathrm{CoO}$ & 0.000000 & 0.000000 & 0.000000 & 0.000000 & 0.000000 & 0.000000 & 0.000000 & 0.000000 \\
\hline $\mathrm{Cr}_{2} \mathrm{O}_{3}$ & 0.000631 & 0.000635 & 0.000666 & 0.000630 & 0.000634 & 0.000633 & 0.000631 & 0.000637 \\
\hline $\mathrm{Cs}_{2} \mathrm{O}$ & 0.000000 & 0.000000 & 0.000000 & 0.000000 & 0.000000 & 0.000000 & 0.000000 & 0.000000 \\
\hline CuO & 0.000000 & 0.000000 & 0.000000 & 0.000000 & 0.000000 & 0.000000 & 0.000000 & 0.000000 \\
\hline $\mathrm{La}_{2} \mathrm{O}_{3}$ & 0.000000 & 0.000000 & 0.000000 & 0.000000 & 0.000000 & 0.000000 & 0.000000 & 0.000000 \\
\hline $\mathrm{MgO}$ & 0.015120 & 0.015230 & 0.015963 & 0.015098 & 0.015197 & 0.015185 & 0.015130 & 0.015259 \\
\hline MnO & 0.007915 & 0.007972 & 0.008356 & 0.007904 & 0.007955 & 0.007949 & 0.007920 & 0.007988 \\
\hline $\mathrm{MoO}_{3}$ & 0.000000 & 0.000000 & 0.000000 & 0.000000 & 0.000000 & 0.000000 & 0.000000 & 0.000000 \\
\hline $\mathrm{Nd}_{2} \mathrm{O}_{3}$ & 0.000000 & 0.000000 & 0.000000 & 0.000000 & 0.000000 & 0.000000 & 0.000000 & 0.000000 \\
\hline $\mathrm{NiO}$ & 0.002292 & 0.002308 & 0.002419 & 0.002288 & 0.002303 & 0.002301 & 0.002293 & 0.002313 \\
\hline $\mathrm{P}_{2} \mathrm{O}_{3}$ & 0.005788 & 0.005831 & 0.006111 & 0.005780 & 0.005818 & 0.005814 & 0.005793 & 0.005842 \\
\hline PdO & 0.000000 & 0.000000 & 0.000000 & 0.000000 & 0.000000 & 0.000000 & 0.000000 & 0.000000 \\
\hline $\mathrm{Pr}_{6} \mathrm{O}_{11}$ & 0.000000 & 0.000000 & 0.000000 & 0.000000 & 0.000000 & 0.000000 & 0.000000 & 0.000000 \\
\hline $\mathrm{RhO}_{2}$ & 0.000000 & 0.000000 & 0.000000 & 0.000000 & 0.000000 & 0.000000 & 0.000000 & 0.000000 \\
\hline $\mathrm{RuO}_{2}$ & 0.000000 & 0.000000 & 0.000000 & 0.000000 & 0.000000 & 0.000000 & 0.000000 & 0.000000 \\
\hline $\mathrm{Sm}_{2} \mathrm{O}_{3}$ & 0.000000 & 0.000000 & 0.000000 & 0.000000 & 0.000000 & 0.000000 & 0.000000 & 0.000000 \\
\hline $\mathrm{SO}_{3}$ & 0.001967 & 0.001981 & 0.002077 & 0.001964 & 0.001977 & 0.001976 & 0.001968 & 0.001985 \\
\hline SrO & 0.001652 & 0.001664 & 0.001744 & 0.001650 & 0.001660 & 0.001659 & 0.001653 & 0.001667 \\
\hline $\mathrm{TiO}_{2}$ & 0.006856 & 0.006906 & 0.007238 & 0.005846 & 0.006891 & 0.006885 & 0.006861 & 0.006919 \\
\hline $\mathrm{U}_{3} \mathrm{O}_{8}$ & 0.000503 & 0.000506 & 0.000531 & 0.000502 & 0.000505 & 0.000505 & 0.000503 & 0.000507 \\
\hline $\mathrm{Y}_{2} \mathrm{O}_{3}$ & 0.000000 & 0.000000 & 0.000000 & 0.000000 & 0.000000 & 0.000000 & 0.000000 & 0.000000 \\
\hline $\mathrm{ZnO}$ & 0.002188 & 0.002204 & 0.002310 & 0.002184 & 0.002199 & 0.002197 & 0.002189 & 0.002208 \\
\hline PCT B & 6.609 & 10.278 & 7.742 & 4.655 & 5.268 & 4.921 & 4.429 & 3.407 \\
\hline PCT $\mathrm{Na}$ & 4.920 & 7.965 & 5.429 & 3.551 & 3.930 & 3.683 & 3.326 & 2.559 \\
\hline PCT $\mathrm{Li}$ & 5.312 & 7.535 & 4.071 & 3.839 & 4.262 & 3.969 & 3.545 & 2.730 \\
\hline
\end{tabular}

* The glass numbers are those used in the reference from which the data were taken. 
Table A.9. WVDP Glass Compositions (mole fractions) and PCT Normalized B, Na, and $\mathrm{Li}$ Releases $\left(\mathrm{g} / \mathrm{m}^{2}\right)$ (continued)

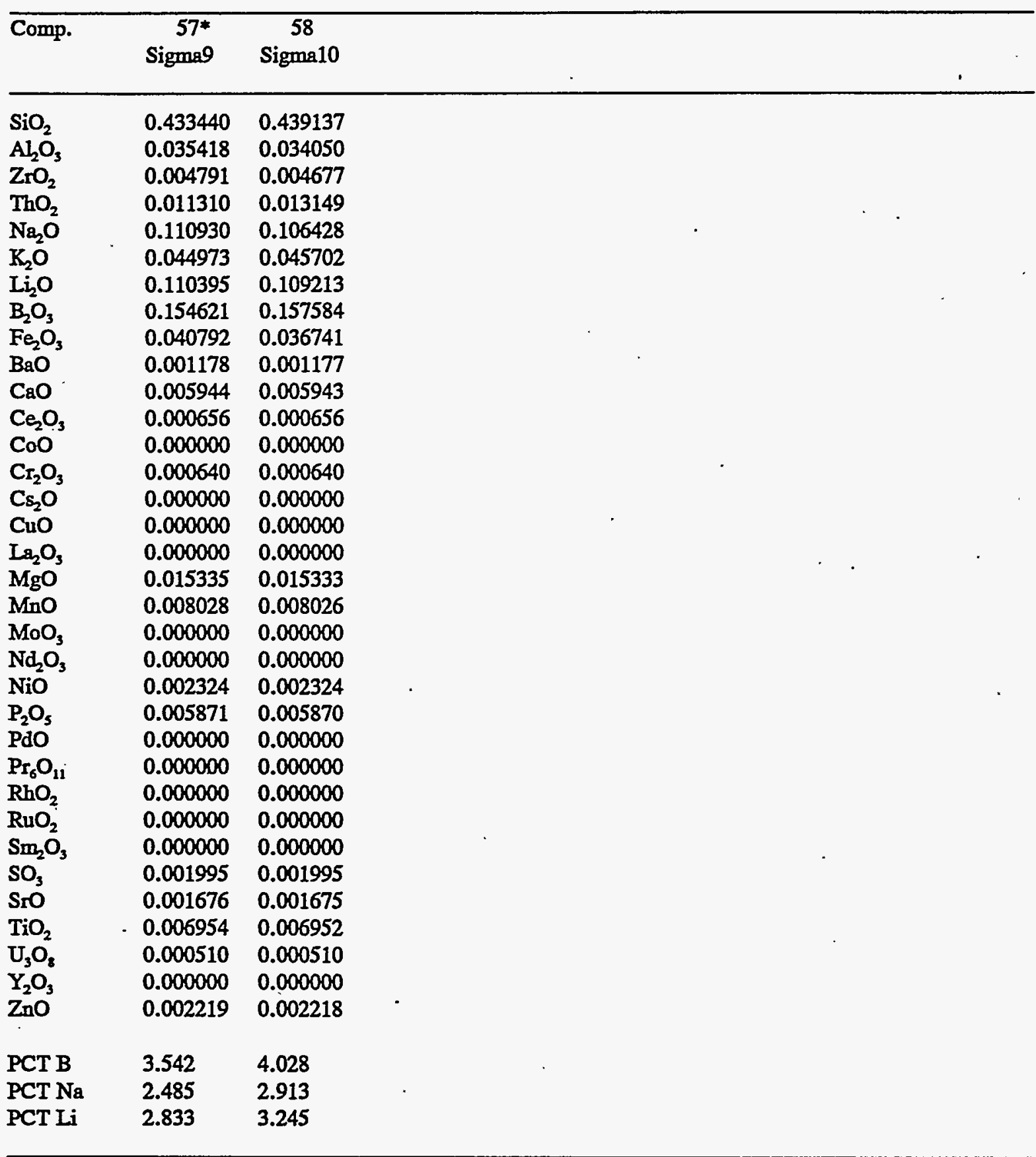

* The glass mumbers are those used in the reference from which the data were taken. 
Table A.10. SRTC Homogeneous Glass Compositions (mole fractions) and PCT Normalized B, Li, and Na Releases $\left(\mathrm{g} / \mathrm{m}^{2}\right)$

\begin{tabular}{|c|c|c|c|c|c|c|c|c|c|c|c|}
\hline & Comp. & HG-1-1 & HG-1-2 & HG-1-3 & HG-2-1 & HG-2-2 & HG-2-3 & HG-3-1 & HG-3-2 & HG-3-3 & AH-165AL \\
\hline \multirow{30}{*}{ · } & $\mathrm{Al}_{2} \mathrm{O}_{3}$ & 0.0344 & 0.0318 & 0.0310 & 0.0319 & 0.0343 & 0.0336 & 0.0352 & 0.0359 & 0.0360 & 0.0848 \\
\hline & $\mathrm{CaO}$ & 0.0152 & 0.0121 & 0.0125 & 0.0123 & 0.0126 & 0.0125 & 0.0130 & 0.0134 & 0.0140 & 0.0059 \\
\hline & $\mathrm{Fe}_{2} \mathrm{O}_{3}$ & 0.0522 & 0.0472 & 0.0465 & 0.0480 & 0.0513 & 0.0503 & 0.0502 & 0.0513 & 0.0496 & 0.0185 \\
\hline & $\mathrm{FeO}$ & 0.0004 & 0.0004 & 0.0013 & 0.0006 & 0.0006 & 0.0006 & 0.0046 & 0.0068 & 0.0093 & 0.0021 \\
\hline & $\mathrm{MgO}$ & 0.0246 & 0.0237 & 0.0237 & 0.0236 & 0.0218 & 0.0218 & 0.0217 & 0.0215 & 0.0217 & 0.0106 \\
\hline & $\mathrm{MnO}$ & 0.0197 & 0.0203 & 0.0200 & 0.0205 & 0.0220 & 0.0216 & 0.0223 & 0.0233 & 0.0224 & 0.0238 \\
\hline & $\mathrm{Na}_{2} \mathrm{O}$ & 0.0954 & 0.0890 & 0.0903 & 0.0901 & 0.1070 & 0.1072 & 0.1042 & 0.1015 & 0.0993 & 0.1103 \\
\hline & $\mathrm{Li}_{2} \mathrm{O}$ & 0.0953 & 0.0973 & 0.0981 & 0.0975 & 0.0945 & 0.0949 & 0.0933 & 0.0918 & 0.0923 & 0.0907 \\
\hline & $\mathrm{NiO}$ & 0.0074 & 0.0066 & 0.0063 & 0.0066 & 0.0066 & 0.0065 & 0.0068 & 0.0067 & 0.0067 & 0.0058 \\
\hline & $\mathrm{SiO}_{2}$ & 0.5601 & 0.5782 & 0.5803 & 0.5811 & 0.5712 & 0.5747 & 0.5680 & 0.5648 & 0.5643 & 0.5754 \\
\hline & $\mathrm{Cr}_{2} \mathrm{O}_{3}$ & 0.0015 & 0.0014 & 0.0012 & 0.0014 & 0.0012 & 0.0013 & 0.0014 & 0.0012 & 0.0010 & 0.0000 \\
\hline & $\mathrm{B}_{2} \mathrm{O}_{3}$ & 0.0650 & 0.0627 & 0.0632 & 0.0619 & 0.0557 & 0.0553 & 0.0565 & 0.0576 & 0.0586 & 0.0680 \\
\hline & SrO & 0.0001 & 0.0002 & 0.0002 & 0.0002 & 0.0002 & 0.0002 & 0.0002 & 0.0002 & 0.0002 & 0.0000 \\
\hline & $\mathrm{ZrO}_{2}$ & 0.0008 & 0.0005 & 0.0000 & 0.0000 & 0.0011 & 0.0004 & 0.0009 & 0.0009 & 0.0008 & 0.0041 \\
\hline & $\mathrm{TiO}_{2}$ & 0.0037 & 0.0022 & 0.0021 & 0.0021 & 0.0018 & 0.0017 & 0.0018 & 0.0019 & 0.0020 & 0.0000 \\
\hline & $\mathrm{K}_{2} \mathrm{O}$ & 0.0183 & 0.0205 & 0.0174 & 0.0165 & 0.0123 & 0.0122 & 0.0137 & 0.0152 & 0.0158 & 0.0000 \\
\hline & $\mathrm{Cs}_{2} \mathrm{O}$ & 0.0002 & 0.0002 & 0.0002 & 0.0001 & 0.0001 & 0.0002 & 0.0001 & 0.0001 & 0.0000 & 0.0000 \\
\hline & $\mathrm{P}_{2} \mathrm{O}_{3}$ & 0.0000 & 0.0002 & 0.0002 & 0.0002 & 0.0003 & 0.0000 & 0.0002 & 0.0001 & 0.0001 & 0.0000 \\
\hline & $\mathrm{Nd}_{2} \mathrm{O}_{3}$ & 0.0000 & 0.0000 & 0.0000 & 0.0000 & 0.0000 & 0.0000 & 0.0000 & 0.0000 & 0.0000 & 0.0000 \\
\hline & $\mathrm{La}_{2} \mathrm{O}_{3}$ & 0.0000 & 0.0000 & 0.0000 & 0.0000 & 0.0000 & 0.0000 & 0.0000 & 0.0000 & 0.0000 & 0.0000 \\
\hline & $\mathrm{BaO}$ & 0.0005 & 0.0003 & 0.0003 & 0.0003 & 0.0004 & 0.0003 & 0.0003 & 0.0004 & 0.0004 & 0.0000 \\
\hline & $\mathrm{PbO}$ & 0.0003 & 0.0006 & 0.0005 & 0.0005 & 0.0007 & 0.0005 & 0.0007 & 0.0007 & 0.0006 & 0.0000 \\
\hline & $\mathrm{Ce}_{2} \mathrm{O}_{3}$ & 0.0000 & 0.0000 & 0.0000 & 0.0000 & 0.0000 & 0.0000 & 0.0000 & 0.0000 & 0.0000 & 0.0000 \\
\hline & $\mathrm{MoO}_{3}$ & 0.0000 & 0.0000 & 0.0000 & 0.0000 & 0.0000 & 0.0000 & 0.0000 & 0.0000 & 0.0000 & 0.0000 \\
\hline & $\mathrm{ZnO}$ & 0.0025 & 0.0030 & 0.0029 & 0.0030 & 0.0032 & 0.0030 & 0.0035 & 0.0031 & 0.0034 & 0.0000 \\
\hline & $\mathrm{CuO}$ & 0.0026 & 0.0017 & 0.0017 & 0.0017 & 0.0012 & 0.0011 & 0.0014 & 0.0015 & 0.0015 & 0.0000 \\
\hline & $\mathbf{Y}_{2} \mathbf{O}_{3}$ & 0.0000 & 0.0000 & .0 .0000 & 0.0000 & 0.0000 & 0.0000 & 0.0000 & 0.0000 & 0.0000 & 0.0000 \\
\hline & PCT B & 0.340 & 0.365 & 0.340 & 0.345 & 0.385 & 0.395 & 0.375 & 0.390 & 0.365 & 0.260 \\
\hline & PCT Li & 0.330 & 0.335 & 0.310 & 0.320 & 0.365 & 0.360 & 0.360 & 0.365 & 0.340 & 0.315 \\
\hline & PCT Na & 0.335 & 0.345 & 0.325 & 0.335 & 0.400 & 0.395 & 0.385 & 0.385 & 0.370 & 0.180 \\
\hline
\end{tabular}

Note: Glass names are those in the reference from which the data were taken, except some names have been shortened. 
Table A.10. SRTC Homogeneous Glass Compositions (mole fractions) and PCT Normalized $\mathrm{B}, \mathrm{Li}$, and $\mathrm{Na}$ Releases $\left(\mathrm{g} / \mathrm{m}^{2}\right)$ (continued)

\begin{tabular}{|c|c|c|c|c|c|c|c|c|c|c|}
\hline Comp. & AH-165AV & AH-165FE & AH-131AL & AH-131AV & AH-131-FE & AH-168AV & AH-168FE & AH-200AL & AH-200AV & $\mathrm{AH}-200 \mathrm{FE}$ \\
\hline $\mathrm{Al}_{2} \mathrm{O}_{3}$ & 0.0325 & 0.0090 & 0.0853 & 0.0280 & 0.0142 & 0.0357 & 0.0156 & 0.0870 & 0.0338 & 0.0135 \\
\hline $\mathrm{CaO}$ & 0.0119 & 0.0161 & 0.0044 & 0.0088 & 0.0116 & 0.0079 & 0.0156 & 0.0064 & 0.0075 & 0.0109 \\
\hline $\mathrm{Fe}_{2} \mathrm{O}_{3}$ & 0.0444 & 0.0366 & 0.0185 & 0.0453 & 0.0307 & 0.0430 & 0.0380 & 0.0182 & 0.0482 & 0.0407 \\
\hline $\mathrm{FeO}$ & 0.0042 & 0.0641 & 0.0008 & 0.0063 & 0.0789 & 0.0056 & 0.0559 & 0.0006 & 0.0036 & 0.0549 \\
\hline $\mathrm{MgO}$ & 0.0105 & 0.0104 & 0.0221 & 0.0108 & 0.0105 & 0.0120 & 0.0114 & 0.0205 & 0.0207 & 0.0199 \\
\hline MnO & 0.0232 & 0.0097 & 0.0228 & 0.0238 & 0.0084 & 0.0243 & 0.0089 & 0.0232 & 0.0241 & 0.0089 \\
\hline $\mathrm{Na}_{2} \mathrm{O}$ & 0.1030 & 0.1113 & 0.1466 & 0.1035 & 0.1132 & 0.1064 & 0.1126 & 0.1133 & 0.1058 & 0.1136 \\
\hline $\mathrm{Li}_{2} \mathrm{O}$ & 0.1076 & 0.0874 & 0.0882 & 0.0926 & 0.0874 & 0.0926 & 0.0891 & 0.0587 & 0.0602 & 0.0576 \\
\hline $\mathrm{NiO}$ & 0.0087 & 0.0256 & 0.0054 & 0.0091 & 0.0221 & 0.0089 & 0.0244 & 0.0054 & 0.0092 & 0.0228 \\
\hline $\mathrm{SiO}_{2}$ & 0.5896 & 0.5580 & 0.4976 & 0.5957 & 0.5506 & 0.5606 & 0.5193 & 0.5334 & 0.5530 & 0.5238 \\
\hline $\mathrm{Cr}_{2} \mathrm{O}_{3}$ & 0.0000 & 0.0000 & 0.0000 & 0.0000 & 0.0000 & 0.0000 & 0.0000 & 0.0000 & 0.0000 & 0.0000 \\
\hline $\mathrm{B}_{2} \mathrm{O}_{3}$ & 0.0605 & 0.0674 & 0.1000 & 0.0710 & 0.0678 & 0.0994 & 0.1058 & 0.0970 & 0.0993 & 0.0963 \\
\hline SrO & 0.0000 & 0.0000 & 0.0000 & 0.0000 & 0.0000 & 0.0000 & 0.0000 & 0.0000 & 0.0000 & 0.0000 \\
\hline $\mathrm{ZrO}_{2}$ & 0.0040 & 0.0044 & 0.0018 & 0.0046 & 0.0045 & 0.0037 & 0.0035 & 0.0002 & 0.0001 & 0.0001 \\
\hline $\mathrm{TiO}_{2}$ & 0.0000 & 0.0000 & 0.0058 & 0.0005 & 0.0001 & 0.0000 & 0.0000 & 0.0141 & 0.0118 & 0.0148 \\
\hline $\mathrm{K}_{2} \mathrm{O}$ & 0.0000 & 0.0000 & 0.0000 & 0.0000 & 0.0000 & 0.0000 & 0.0000 & 0.0219 & 0.0227 & 0.0222 \\
\hline $\mathrm{Cs}_{2} \mathrm{O}$ & 0.0000 & 0.0000 & 0.0000 & 0.0000 & 0.0000 & 0.0000 & 0.0000 & 0.0000 & 0.0000 & 0.0000 \\
\hline $\mathrm{P}_{2} \mathrm{O}_{5}$ & 0.0000 & 0.0000 & 0.0000 & 0.0000 & 0.0000 & 0.0000 & 0.0000 & 0.0000 & 0.0000 & 0.0000 \\
\hline $\mathrm{Nd}_{2} \mathrm{O}_{3}$ & 0.0000 & 0.0000 & 0.0000 & 0.0000 & 0.0000 & 0.0000 & 0.0000 & 0.0000 & 0.0000 & 0.0000 \\
\hline $\mathrm{La}_{2} \mathrm{O}_{3}$ & 0.0000 & 0.0000 & 0.0007 & 0.0000 & 0.0000 & 0.0000 & 0.0000 & 0.0000 & 0.0000 & 0.0000 \\
\hline $\mathrm{BaO}$ & 0.0000 & 0.0000 & 0.0000 & 0.0000 & 0.0000 & 0.0000 & 0.0000 & 0.0000 & 0.0000 & 0.0000 \\
\hline $\mathrm{PbO}$ & 0.0000 & 0.0000 & 0.0000 & 0.0000 & 0.0000 & 0.0000 & 0.0000 & 0.0000 & 0.0000 & 0.0000 \\
\hline $\mathrm{Ce}_{2} \mathrm{O}_{3}$ & 0.0000 & 0.0000 & 0.0000 & 0.0000 & 0.0000 & 0.0000 & 0.0000 & 0.0000 & 0.0000 & 0.0000 \\
\hline $\mathrm{MoO}_{3}$ & 0.0000 & 0.0000 & 0.0000 & 0.0000 & 0.0000 & 0.0000 & 0.0000 & 0.0000 & 0.0000 & 0.0000 \\
\hline $\mathrm{ZnO}$ & 0.0000 & 0.0000 & 0.0000 & 0.0000 & 0.0000 & 0.0000 & 0.0000 & 0.0000 & 0.0000 & 0.0000 \\
\hline $\mathrm{CuO}$ & 0.0000 & 0.0000 & 0.0000 & 0.0000 & 0.0000 & 0.0000 & 0.0000 & 0.0000 & 0.0000 & 0.0000 \\
\hline $\mathrm{Y}_{2} \mathrm{O}_{3}$ & 0.0000 & 0.0000 & 0.0000 & 0.0000 & 0.0000 & 0.0000 & 0.0000 & 0.0000 & 0.0000 & 0.0000 \\
\hline PCT B & 0.320 & 2.380 & 0.360 & 0.340 & 1.790 & 0.400 & 2.565 & 0.230 & 0.290 & 2.550 \\
\hline PCT Li & 0.330 & 1.965 & 0.350 & 0.365 & 1.600 & 0.375 & 2.210 & 0.285 & 0.310 & 2.190 \\
\hline PCT Na & 0.265 & 2.090 & 0.345 & 0.305 & 1.545 & 0.295 & 1.950 & 0.220 & 0.295 & 2.190 \\
\hline
\end{tabular}

Note: Glass names are those in the reference from which the data were taken, except some names have been shortened. 
Table A.10. SRTC Homogeneous Glass Compositions (mole fractions) and PCT Normalized B, $\mathrm{Li}$, and $\mathrm{Na}$ Releases $\left(\mathrm{g} / \mathrm{m}^{2}\right)$ (continued)

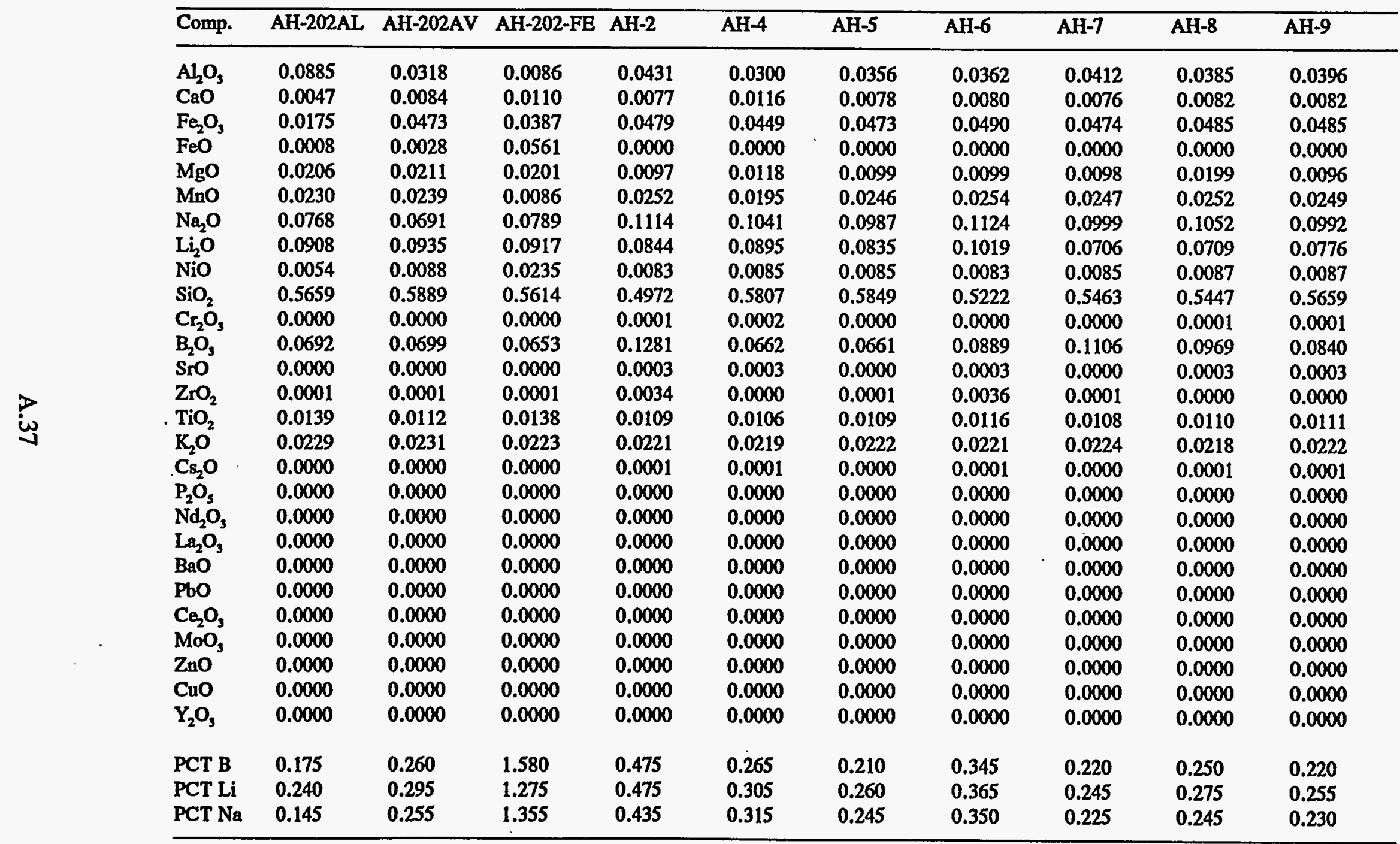

Note: Glass names are those in the reference from which the data were taken, except some names have been shortened. 
Table A.10. SRTC Homogeneous Glass Compositions (mole fractions) and PCT Normalized B, Li, and Na Releases $\left(\mathrm{g} / \mathrm{m}^{2}\right)$ (continued)

\begin{tabular}{|c|c|c|c|c|c|c|c|c|c|c|}
\hline Comp. & AH-10 & AH-11 & AH-12 & AH-13 & AH-14 & AH-15 & AH-16 & AH-17 & SFRIT1 & SFRIT2 \\
\hline $\mathrm{Al}_{2} \mathrm{O}_{3}$ & 0.0329 & 0.0373 & 0.0396 & 0.0437 & 0.0475 & 0.0465 & 0.0420 & 0.0371 & 0.0304 & 0.0304 \\
\hline $\mathrm{CaO}$ & 0.0079 & 0.0077 & 0.0082 & 0.0153 & 0.0152 & 0.0156 & 0.0151 & 0.0077 & 0.0177 & 0.0177 \\
\hline $\mathrm{Fe}_{2} \mathrm{O}_{3}$ & 0.0462 & 0.0460 & 0.0485 & 0.0586 & 0.0595 & 0.0588 & 0.0565 & 0.0472 & 0.0600 & 0.0600 \\
\hline $\mathrm{FeO}$ & 0.0000 & 0.0000 & 0.0000 & 0.0000 & 0.0000 & 0.0000 & 0.0000 & 0.0000 & 0.0000 & 0.0000 \\
\hline MgO & 0.0180 & 0.0184 & 0.0096 & 0.0084 & 0.0090 & 0.0175 & 0.0167 & 0.0095 & 0.0141 & 0.0141 \\
\hline $\mathrm{MnO}$ & 0.0246 & 0.0247 & 0.0249 & 0.0315 & 0.0322 & 0.0317 & 0.0306 & 0.0252 & 0.0184 & 0.0184 \\
\hline $\mathrm{Na}_{2} \mathrm{O}$ & 0.0720 & 0.0691 & 0.0992 & 0.0976 & 0.1077 & 0.1048 & 0.0711 & 0.0705 & 0.1254 & 0.1254 \\
\hline $\mathrm{Li}_{2} \mathrm{O}$ & 0.0971 & 0.0809 & 0.0776 & 0.0764 & 0.0902 & 0.0647 & 0.0916 & 0.1037 & 0.0733 & 0.0733 \\
\hline $\mathrm{NiO}$ & 0.0084 & 0.0083 & 0.0087 & 0.0105 & 0.0103 & 0.0104 & 0.0099 & 0.0081 & 0.0100 & 0.0100 \\
\hline $\mathrm{SiO}_{2}$ & 0.5892 & 0.5600 & 0.5660 & 0.5607 & 0.5098 & 0.5238 & 0.5630 & 0.5774 & 0.5376 & 0.5376 \\
\hline $\mathrm{Cr}_{2} \mathrm{O}_{3}$ & 0.0000 & 0.0001 & 0.0000 & 0.0000 & 0.0000 & 0.0001 & 0.0003 & 0.0004 & 0.0004 & 0.0004 \\
\hline $\mathrm{B}_{2} \mathrm{O}_{3}$ & 0.0712 & 0.1151 & 0.0840 & 0.0633 & 0.0810 & 0.0912 & 0.0697 & 0.0771 & 0.0824 & 0.0824 \\
\hline SrO & 0.0003 & 0.0003 & 0.0003 & 0.0004 & 0.0004 & 0.0004 & 0.0005 & 0.0002 & 0.0000 & 0.0000 \\
\hline $\mathrm{ZrO}_{2}$ & 0.0000 & 0.0000 & 0.0000 & 0.0001 & 0.0032 & 0.0000 & 0.0000 & 0.0035 & 0.0006 & 0.0006 \\
\hline $\mathrm{TiO}_{2}$ & 0.0106 & 0.0108 & 0.0111 & 0.0111 & 0.0113 & 0.0116 & 0.0110 & 0.0108 & 0.0100 & 0.0100 \\
\hline $\mathrm{K}_{2} \mathrm{O}$ & 0.0214 & 0.0212 & 0.0222 & 0.0223 & 0.0224 & 0.0227 & 0.0219 & 0.0215 & 0.0193 & 0.0193 \\
\hline $\mathrm{Cs}_{2} \mathrm{O}$ & 0.0001 & 0.0001 & 0.0001 & 0.0001 & 0.0002 & 0.0001 & 0.0001 & 0.0001 & 0.0000 & 0.0000 \\
\hline $\mathrm{P}_{2} \mathrm{O}_{5}$ & 0.0000 & 0.0000 & 0.0000 & 0.0000 & 0.0000 & 0.0000 & 0.0000 & 0.0000 & 0.0000 & 0.0000 \\
\hline $\mathrm{Nd}_{2} \mathrm{O}_{3}$ & .0 .0000 & 0.0000 & 0.0000 & 0.0000 & 0.0000 & 0.0000 & 0.0000 & 0.0000 & 0.0000 & 0.0000 \\
\hline $\mathrm{La}_{2} \mathrm{O}_{3}$ & 0.0000 & 0.0000 & 0.0000 & 0.0000 & 0.0000 & 0.0000 & 0.0000 & 0.0000 & 0.0000 & 0.0000 \\
\hline $\mathrm{BaO}$ & 0.0000 & 0.0000 & 0.0000 & 0.0000 & 0.0000 & 0.0000 & 0.0000 & 0.0000 & 0.0004 & 0.0004 \\
\hline $\mathrm{PbO}$ & 0.0000 & 0.0000 & 0.0000 & 0.0000 & 0.0000 & 0.0000 & 0.0000 & 0.0000 & 0.0000 & 0.0000 \\
\hline $\mathrm{Ce}_{2} \mathrm{O}_{3}$ & 0.0000 & 0.0000 & 0.0000 & 0.0000 & 0.0000 & 0.0000 & 0.0000 & 0.0000 & 0.0000 & 0.0000 \\
\hline $\mathrm{MoO}_{3}$ & 0.0000 & 0.0000 & 0.0000 & 0.0000 & 0.0000 & 0.0000 & 0.0000 & 0.0000 & 0.0000 & 0.0000 \\
\hline $\mathrm{ZnO}$ & 0.0000 & 0.0000 & 0.0000 & 0.0000 & 0.0000 & 0.0000 & 0.0000 & 0.0000 & 0.0000 & 0.0000 \\
\hline $\mathrm{CuO}$ & 0.0000 & 0.0000 & 0.0000 & 0.0000 & 0.0000 & 0.0000 & 0.0000 & 0.0000 & 0.0000 & 0.0000 \\
\hline $\mathbf{Y}_{2} \mathrm{O}_{3}$ & 0.0000 & 0.0000 & 0.0000 & 0.0000 & 0.0000 & 0.0000 & 0.0000 & 0.0000 & 0.0000 & 0.0000 \\
\hline PCT B & 0.215 & 0.235 & 0.425 & 0.215 & 0.260 & 0.215 & 0.210 & 0.180 & 0.375 & 0.365 \\
\hline PCT Li & 0.260 & 0.285 & 0.305 & 0.260 & 0.290 & 0.240 & 0.240 & 0.225 & 0.345 & 0.350 \\
\hline PCT Na & 0.215 & 0.225 & 0.365 & 0.235 & 0.305 & 0.225 & 0.220 & 0.185 & 0.355 & 0.350 \\
\hline
\end{tabular}

Note: Glass names are those in the reference from which the data were taken, except some names have been shortened. 
Table A.10. SRTC Homogeneous Glass Compositions (mole fractions) and PCT Normalized $\mathrm{B}, \mathrm{Li}$, and Na Releases $\left(\mathrm{g} / \mathrm{m}^{2}\right)$ (continued)

\begin{tabular}{|c|c|c|c|c|c|c|c|c|c|c|}
\hline Comp. & SFRIT3 & $202 P$ & $202 G$ & $200 R$ & NBS623 & $165 \mathrm{CGW}$ & ARM1-4/88 & ARM1-5/89 & ARM1-7/90 & ARM1-12/90 \\
\hline $\mathrm{Al}_{2} \mathrm{O}_{3}$ & 0.0304 & 0.0274 & 0.0291 & 0.0311 & 0.0394 & 0.0264 & 0.0369 & 0.0369 & 0.0369 & 0.0369 \\
\hline $\mathrm{CaO}$ & 0.0177 & 0.0141 & 0.0149 & 0.0180 & 0.0080 & 0.0176 & 0.0268 & 0.0268 & 0.0268 & 0.0268 \\
\hline $\mathrm{Fe}_{2} \mathrm{O}_{3}$ & 0.0600 & 0.0436 & 0.0487 & 0.0529 & 0.0000 & 0.0520 & 0.0000 & 0.0000 & 0.0000 & 0.0000 \\
\hline $\mathrm{FeO}$ & 0.0000 & 0.0000 & 0.0000 & 0.0000 & 0.0000 & 0.0000 & 0.0000 & 0.0000 & 0.0000 & 0.0000 \\
\hline $\mathrm{MgO}$ & 0.0141 & 0.0471 & 0.0508 & 0.0213 & 0.0000 & .0 .0163 & 0.0000 & 0.0000 & 0.0000 & 0.0000 \\
\hline Mno & 0.0184 & 0.0000 & 0.0000 & 0.0206 & 0.0000 & 0.0232 & 0.0000 & 0.0000 & 0.0000 & 0.0000 \\
\hline $\mathrm{Na}_{2} \mathrm{O}$ & 0.1254 & 0.0965 & 0.0855 & 0.1473 & 0.0659 & 0.1082 & 0.1051 & 0.1051 & 0.1051 & 0.1051 \\
\hline $\mathrm{Li}_{2} \mathrm{O}$ & 0.0733 & 0.0744 & 0.0811 & 0.0692 & 0.0000 & 0.1075 & 0.1145 & 0.1145 & 0.1145 & 0.1145 \\
\hline NiO & 0.0100 & 0.0061 & 0.0058 & 0.0049 & 0.0000 & 0.0083 & 0.0000 & 0.0000 & 0.0000 & 0.0000 \\
\hline $\mathrm{SiO}_{2}$ & 0.5376 & 0.5540 & 0.6000 & 0.5157 & 0.7754 & 0.5758 & 0.5213 & 0.5213 & 0.5213 & 0.5213 \\
\hline $\mathrm{Cr}_{2} \mathrm{O}_{3}$ & 0.0004 & 0.0003 & 0.0003 & 0.0000 & 0.0000 & 0.0000 & 0.0000 & 0.0000 & 0.0000 & 0.0000 \\
\hline $\mathrm{B}_{2} \mathrm{O}_{3}$ & 0.0824 & 0.0803 & 0.0557 & 0.0934 & 0.0981 & 0.0619 & 0.1093 & 0.1093 & 0.1093 & 0.1093 \\
\hline Sro & 0.0000 & 0.0000 & 0.0000 & 0.0000 & 0.0000 & 0.0000 & 0.0029 & 0.0029 & 0.0029 & 0.0029 \\
\hline $\mathrm{ZrO}_{2}$ & 0.0006 & 0.0000 & 0.0000 & 0.0000 & 0.0000 & 0.0026 & 0.0098 & 0.0098 & 0.0098 & .0 .0098 \\
\hline $\mathrm{TiO}_{2}$ & 0.0100 & 0.0101 & 0.0055 & 0.0000 & 0.0000 & 0.0000 & 0.0271 & 0.0271 & 0.0271 & 0.0271 \\
\hline $\mathrm{K}_{2} \mathrm{O}$ & 0.0193 & 0.0395 & 0.0187 & 0.0255 & 0.0041 & 0.0000 & 0.0000 & 0.0000 & 0.0000 & 0.0000 \\
\hline $\mathrm{Cs}_{2} \mathrm{O}$ & 0.0000 & 0.0003 & 0.0001 & 0.0000 & 0.0000 & 0.0000 & 0.0028 & 0.0028 & 0.0028 & 0.0028 \\
\hline $\mathrm{P}_{2} \mathrm{O}_{3}$ & 0.0000 & 0.0000 & 0.0000 & 0.0000 & 0.0000 & 0.0000 & 0.0031 & 0.0031 & 0.0031 & 0.0031 \\
\hline $\mathrm{Nd}_{2} \mathrm{O}_{3}$ & 0.0000 & 0.0000 & 0.0000 & 0.0000 & 0.0000 & 0.0000 & 0.0119 & 0.0119 & 0.0119 & 0.0119 \\
\hline $\mathrm{La}_{2} \mathrm{O}_{3}$ & 0.0000 & 0.0000 & 0.0000 & 0.0000 & 0.0000 & 0.0000 & 0.0000 & 0.0000 & 0.0000 & 0.0000 \\
\hline $\mathrm{BaO}$ & 0.0004 & 0.0008 & 0.0007 & 0.0000 & 0.0092 & 0.0000 & 0.0029 & 0.0029 & 0.0029 & 0.0029 \\
\hline $\mathrm{PbO}$ & 0.0000 & 0.0000 & 0.0000 & 0.0000 & 0.0000 & 0.0000 & 0.0000 & 0.0000 & 0.0000 & 0.0000 \\
\hline $\mathrm{Ce}_{2} \mathrm{O}_{3}$ & 0.0000 & 0.0000 & 0.0000 & 0.0000 & 0.0000 & 0.0000 & 0.0056 & 0.0056 & 0.0056 & 0.0056 \\
\hline $\mathrm{MoO}_{3}$ & 0.0000 & 0.0000 & 0.0000 & 0.0000 & 0.0000 & 0.0000 & 0.0078 & 0.0078 & 0.0078 & 0.0078 \\
\hline $\mathrm{ZnO}$ & 0.0000 & 0.0000 & 0.0000 & 0.0000 & 0.0000 & 0.0000 & 0.0121 & 0.0121 & 0.0121 & 0.0121 \\
\hline $\mathrm{CuO}$ & 0.0000 & 0.0054 & 0.0032 & 0.0000 & 0.0000 & 0.0000 & 0.0000 & 0.0000 & 0.0000 & 0.0000 \\
\hline $\mathrm{Y}_{2} \mathrm{O}_{3}$ & 0.0000 & 0.0000 & 0.0000 & 0.0000 & 0.0000 & 0.0000 & 0.0000 & 0.0000 & 0.0000 & 0.0000 \\
\hline PCT B & 0.380 & 0.320 & 0.195 & 0.575 & 0.055 & 0.420 & 0.290 & 0.295 & 0.240 & 0.250 \\
\hline PCT Li & 0.410 & 0.255 & 0.195 & 0.485 & 0.005 & 0.365 & 0.375 & 0.280 & 0.285 & 0.295 \\
\hline PCT Na & 0.435 & 0.370 & 0.260 & 0.505 & 0.075 & 0.410 & 0.345 & 0.235 & 0.255 & 0.260 \\
\hline
\end{tabular}

Note: Glass names are those in the reference from which the data were taken, except some names have been shortened. 


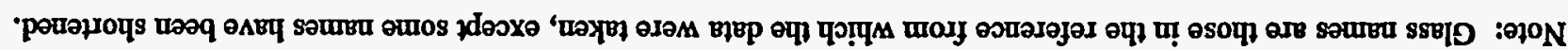

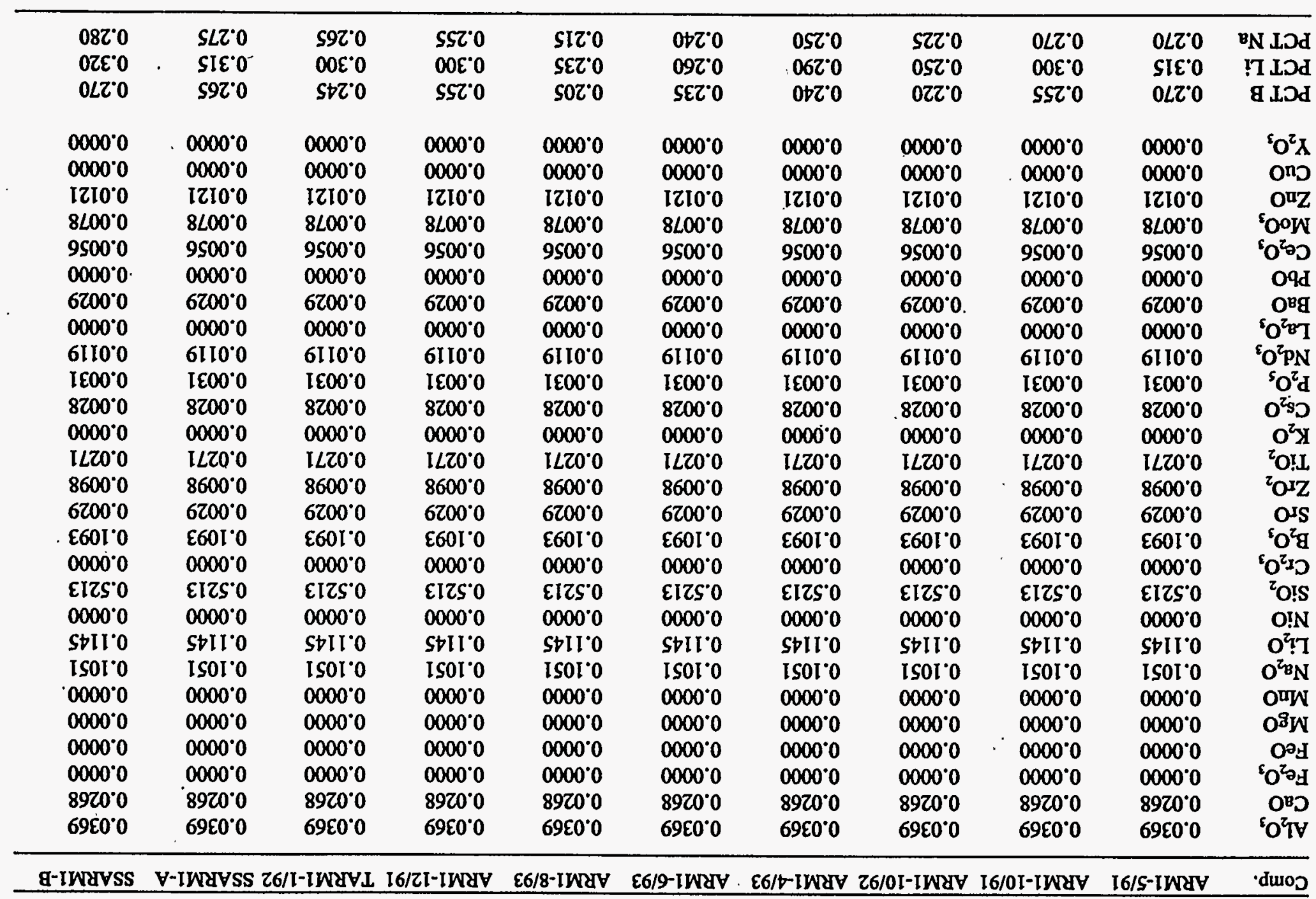

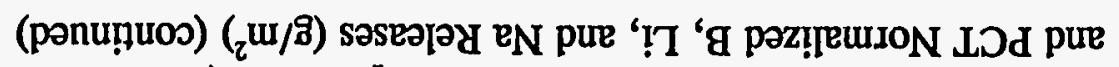

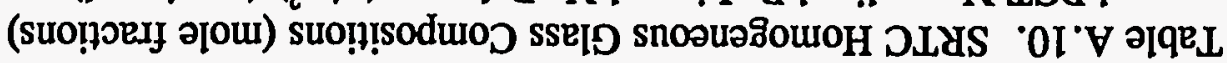


Tạble A. 10. SRTC Homogeneous Glass Compositions (mole fractions)

and PCT Normalized $\mathrm{B}, \mathrm{Li}$, and Na Releases $\left(\mathrm{g} / \mathrm{m}^{2}\right)$ (continued)

\begin{tabular}{|c|c|c|c|c|c|c|c|c|c|}
\hline Comp. & EA1-7A & EA-1-7B & EA-2-7 & EA-7 & T-EA & SS-EA-19 & SS-EA-15 & SS-EA-1-7 & SS-EA-2-7 \\
\hline $\mathrm{Al}_{2} \mathrm{O}_{3}$ & 0.0228 & 0.0228 & 0.0228 & 0.0228 & 0.0228 & 0.0228 & 0.0228 & 0.0228 & 0.0228 \\
\hline $\mathrm{CaO}$ & 0.0125 & 0.0125 & 0.0125 & 0.0125 & 0.0125 & 0.0125 & 0.0125 & 0.0125 & 0.0125 \\
\hline $\mathrm{Fe}_{2} \mathrm{O}_{3}$ & 0.0290 & 0.0290 & 0.0290 & 0.0290 & 0.0290 & 0.0290 & 0.0290 & 0.0290 & 0.0290 \\
\hline $\mathrm{FeO}$ & 0.0127 & 0.0127 & 0.0127 & 0.0127 & 0.0127 & 0.0127 & 0.0127 & 0.0127 & 0.0127 \\
\hline $\mathrm{MgO}$ & 0.0268 & 0.0268 & 0.0268 & 0.0268 & 0.0268 & 0.0268 & 0.0268 & 0.0268 & 0.0268 \\
\hline MnO & 0.0119 & 0.0119 & 0.0119 & 0.0119 & 0.0119 & 0.0119 & 0.0119 & 0.0119 & 0.0119 \\
\hline $\mathrm{Na}_{2} \mathrm{O}$ & 0.1702 & 0.1702 & 0.1702 & 0.1702 & 0.1702 & 0.1702 & 0.1702 & 0.1702 & 0.1702 \\
\hline $\mathrm{Li}_{2} \mathrm{O}$ & 0.0895 & 0.0895 & 0.0895 & 0.0895 & 0.0895 & 0.0895 & 0.0895 & 0.0895 & 0.0895 \\
\hline NiO & 0.0048 & 0.0048 & 0.0048 & 0.0048 & 0.0048 & 0.0048 & 0.0048 & 0.0048 & 0.0048 \\
\hline $\mathrm{SiO}_{2}$ & 0.5091 & 0.5091 & 0.5091 & 0.5091 & 0.5091 & 0.5091 & 0.5091 & 0.5091 & 0.5091 \\
\hline $\mathrm{Cr}_{2} \mathrm{O}_{3}$ & 0.0000 & 0.0000 & 0.0000 & 0.0000 & 0.0000 & 0.0000 & 0.0000 & 0.0000 & 0.0000 \\
\hline $\mathrm{B}_{2} \mathrm{O}_{3}$ & 0.1019 & 0.1019 & 0.1019 & 0.1019 & 0.1019 & 0.1019 & 0.1019 & 0.1019 & 0.1019 \\
\hline SrO & 0.0000 & 0.0000 & 0.0000 & 0.0000 & 0.0000 & 0.0000 & 0.0000 & 0.0000 & 0.0000 \\
\hline $\mathrm{ZrO}_{2}$ & 0.0023 & 0.0023 & 0.0023 & 0.0023 & 0.0023 & 0.0023 & 0.0023 & 0.0023 & 0.0023 \\
\hline $\mathrm{TiO}_{2}$ & 0.0055 & 0.0055 & 0.0055 & 0.0055 & 0.0055 & 0.0055 & 0.0055 & 0.0055 & 0.0055 \\
\hline $\mathrm{K}_{2} \mathrm{O}$ & 0.0003 & 0.0003 & 0.0003 & 0.0003 & 0.0003 & 0.0003 & 0.0003 & 0.0003 & 0.0003 \\
\hline $\mathrm{Cs}_{2} \mathrm{O}$ & 0.0000 & 0.0000 & 0.0000 & 0.0000 & 0.0000 & 0.0000 & 0.0000 & 0.0000 & 0.0000 \\
\hline $\mathrm{P}_{2} \mathrm{O}_{5}$ & 0.0000 & 0.0000 & 0.0000 & 0.0000 & 0.0000 & 0.0000 & 0.0000 & 0.0000 & 0.0000 \\
\hline $\mathrm{Nd}_{2} \mathrm{O}_{3}$ & 0.0000 & 0.0000 & 0.0000 & 0.0000 & 0.0000 & 0.0000 & 0.0000 & 0.0000 & 0.0000 \\
\hline $\mathrm{La}_{2} \mathrm{O}_{3}$ & 0.0008 & 0.0008 & 0.0008 & 0.0008 & 0.0008 & 0.0008 & 0.0008 & 0.0008 & 0.0008 \\
\hline $\mathrm{BaO}$ & 0.0000 & 0.0000 & 0.0000 & 0.0000 & 0.0000 & 0.0000 & 0.0000 & 0.0000 & 0.0000 \\
\hline PbO & 0.0000 & 0.0000 & 0.0000 & 0.0000 & 0.0000 & 0.0000 & 0.0000 & 0.0000 & 0.0000 \\
\hline $\mathrm{Ce}_{2} \mathrm{O}_{3}$ & 0.0000 & 0.0000 & 0.0000 & 0.0000 & 0.0000 & 0.0000 & 0.0000 & 0.0000 & 0.0000 \\
\hline $\mathrm{MoO}_{3}$ & 0.0000 & 0.0000 & 0.0000 & 0.0000 & 0.0000 & 0.0000 & 0.0000 & 0.0000 & 0.0000 \\
\hline $\mathrm{ZnO}$ & 0.0000 & 0.0000 & 0.0000 & 0.0000 & 0.0000 & 0.0000 & 0.0000 & 0.0000 & 0.0000 \\
\hline $\mathrm{CuO}$ & 0.0000 & 0.0000 & 0.0000 & 0.0000 & 0.0000 & 0.0000 & 0.0000 & 0.0000 & 0.0000 \\
\hline $\mathrm{Y}_{2} \mathrm{O}_{3}$ & 0.0000 & 0.0000 & 0.0000 & 0.0000 & 0.0000 & 0.0000 & 0.0000 & 0.0000 & 0.0000 \\
\hline PCT B & 7.000 & 8.395 & 7.605 & 8.860 & 7.375 & 8.205 & 8.395 & 9.250 & 8.880 \\
\hline PCT Li & 3.925 & 5.345 & 4.715 & 4.945 & 4.260 & 4.560 & 4.870 & 5.140 & 4.885 \\
\hline $\mathrm{PCT} \mathrm{Na}$ & 5.760 & 7.245 & 6.390 & 6.945 & 5.910 & 6.545 & 6.800 & 7.165 & 6.860 \\
\hline
\end{tabular}

Note: Glass names are those in the reference from which the data were taken, except some names have been shortened. 
Table A.10. SRTC Homogeneous Glass Compositions (mole fractions) and PCT Normalized $\mathrm{B}, \mathrm{Li}$, and $\mathrm{Na}$ Releases $\left(\mathrm{g} / \mathrm{m}^{2}\right)$ (continued)

\begin{tabular}{|c|c|c|c|c|c|c|c|c|c|c|}
\hline Comp. & SRS-SEA-A & SRS-SEA-B & CUASEA-A & CUASEA-B & TDS131-EA & TDS131-SOP & BLEND1-7A & BLEND1-7B & BLEND1.6 & BATCH1-7A \\
\hline $\mathrm{Al}_{2} \mathrm{O}_{3}$ & 0.0228 & 0.0228 & 0.0228 & 0.0228 & 0.0371 & 0.0350 & 0.0266 & 0.0266 & 0.0266 & 0.0316 \\
\hline $\mathrm{CaO}$ & 0.0125 & 0.0125 & 0.0125 & 0.0125 & 0.0108 & 0.0106 & 0.0120 & 0.0120 & 0.0120 & 0.0144 \\
\hline $\mathrm{Fe}_{2} \mathrm{O}_{3}$ & 0.0290 & 0.0290 & 0.0290 & 0.0290 & 0.0588 & 0.0462 & 0.0445 & 0.0445 & 0.0445 & 0.0531 \\
\hline $\mathrm{FeO}$ & 0.0127 & 0.0127 & 0.0127 & 0.0127 & 0.0000 & 0.0000 & 0.0000 & 0.0000 & 0.0000 & 0.0000 \\
\hline $\mathrm{MgO}$ & 0.0268 & 0.0268 & 0.0268 & 0.0268 & 0.0249 & 0.0226 & 0.0228 & 0.0228 & 0.0228 & 0.0233 \\
\hline $\mathrm{MnO}$ & 0.0119 & 0.0119 & 0.0119 & 0.0119 & 0.0293 & 0.0307 & 0.0154 & 0.0154 & 0.0154 & 0.0160 \\
\hline $\mathrm{Na}_{2} \mathrm{O}$ & 0.1702 & 0.1702 & 0.1702 & 0.1702 & 0.1546 & 0.1431 & 0.0960 & 0.0960 & 0.0960 & 0.0960 \\
\hline $\mathrm{Li}_{2} \mathrm{O}$ & 0.0895 & 0.0895 & 0.0895 & 0.0895 & 0.1061 & 0.0912 & 0.0969 & 0.0969 & 0.0969 & 0.0980 \\
\hline $\mathrm{NiO}$ & 0.0048 & 0.0048 & 0.0048 & 0.0048 & 0.0127 & 0.0133 & 0.0078 & 0.0078 & 0.0078 & 0.0066 \\
\hline $\mathrm{SiO}_{2}$ & 0.5091 & 0.5091 & 0.5091 & 0.5091 & 0.4348 & 0.4977 & 0.5630 & 0.5630 & 0.5630 & 0.5522 \\
\hline $\mathrm{Cr}_{2} \mathrm{O}_{3}$ & 0.0000 & 0.0000 & 0.0000 & 0.0000 & 0.0002 & 0.0005 & 0.0006 & 0.0006 & 0.0006 & 0.0005 \\
\hline $\mathrm{B}_{2} \mathrm{O}_{3}$ & 0.1019 & 0.1019 & 0.1019 & 0.1019 & 0.1197 & 0.1004 & 0.0754 & 0.0754 & 0.0754 & 0.0739 \\
\hline Sro & 0.0000 & 0.0000 & 0.0000 & 0.0000 & 0.0000 & 0.0000 & 0.0000 & 0.0000 & 0.0000 & 0.0000 \\
\hline $\mathrm{ZrO}_{2}$ & 0.0023 & 0.0023 & 0.0023 & 0.0023 & 0.0023 & 0.0018 & 0.0007 & 0.0007 & 0.0007 & 0.0005 \\
\hline $\mathrm{TiO}_{2}$ & 0.0055 & 0.0055 & 0.0055 & 0.0055 & 0.0076 & 0.0060 & 0.0073 & 0.0073 & 0.0073 & 0.0056 \\
\hline $\mathrm{K}_{2} \mathrm{O}$ & 0.0003 & 0.0003 & 0.0003 & 0.0003 & 0.0003 & 0.0003 & 0.0255 & 0.0255 & 0.0255 & 0.0234 \\
\hline $\mathrm{Cs}_{2} \mathrm{O}$ & 0.0000 & 0.0000 & 0.0000 & 0.0000 & 0.0000 & 0.0000 & 0.0002 & 0.0002 & 0.0002 & 0.0001 \\
\hline $\mathrm{P}_{2} \mathrm{O}_{3}$ & 0.0000 & 0.0000 & 0.0000 & 0.0000 & 0.0000 & 0.0000 & 0.0000 & 0.0000 & 0.0000 & 0.0000 \\
\hline $\mathrm{Nd}_{2} \mathrm{O}_{3}$ & 0.0000 & 0.0000 & 0.0000 & 0.0000 & 0.0000 & 0.0000 & 0.0004 & 0.0004 & 0.0004 & 0.0003 \\
\hline $\mathrm{La}_{2} \mathrm{O}_{3}$ & 0.0008 & 0.0008 & 0.0008 & 0.0008 & 0.0007 & 0.0007 & 0.0000 & 0.0000 & 0.0000 & 0.0000 \\
\hline $\mathrm{BaO}$ & 0.0000 & 0.0000 & 0.0000 & 0.0000 & 0.0000 & 0.0000 & 0.0008 & 0.0008 & 0.0008 & 0.0006 \\
\hline $\mathrm{PbO}$ & 0.0000 & 0.0000 & 0.0000 & 0.0000 & 0.0000 & 0.0000 & 0.0000 & 0.0000 & 0.0000 & 0.0000 \\
\hline $\mathrm{Ce}_{2} \mathrm{O}_{3}$ & 0.0000 & 0.0000 & 0.0000 & 0.0000 & 0.0000 & 0.0000 & 0.0000 & 0.0000 & 0.0000 & 0.0000 \\
\hline $\mathrm{MoO}_{3}$ & 0.0000 & 0.0000 & 0.0000 & 0.0000 & 0.0000 & 0.0000 & 0.0007 & 0.0007 & 0.0007 & 0.0005 \\
\hline $\mathrm{ZnO}$ & 0.0000 & 0.0000 & 0.0000 & 0.0000 & 0.0000 & 0.0000 & 0.0000 & 0.0000 & 0.0000 & 0.0000 \\
\hline $\mathrm{CuO}$ & 0.0000 & 0.0000 & 0.0000 & 0.0000 & 0.0000 & 0.0000 & 0.0036 & 0.0036 & 0.0036 & 0.0033 \\
\hline $\mathbf{Y}_{2} \mathrm{O}_{3}$ & 0.0000 & 0.0000 & 0.0000 & 0.0000 & 0.0000 & 0.0000 & 0.0000 & 0.0000 & 0.0000 & 0.0000 \\
\hline PCT B & 8.225 & 8.495 & 8.425 & 8.230 & 2.215 & 1.405 & 0.360 & 0.390 & 0.360 & 0.365 \\
\hline PCT Li & 4.670 & 4.560 & 4.835 & 4.605 & 1.925 & 1.225 & 0.405 & 0.400 & 0.380 & 0.405 \\
\hline PCT Na & 6.425 & 6.755 & 6.475 & 6.710 & 2.140 & 1.150 & 0.390 & 0.395 & 0.375 & 0.400 \\
\hline
\end{tabular}

Note: Glass names are those in the reference from which the data were taken, except some names have been shortened. 
Table A.10. SRTC Homogeneous Glass Compositions (mole fractions) and PCT Normalized B, Li, and Na Releases $\left(\mathrm{g} / \mathrm{m}^{2}\right)$ (continued)

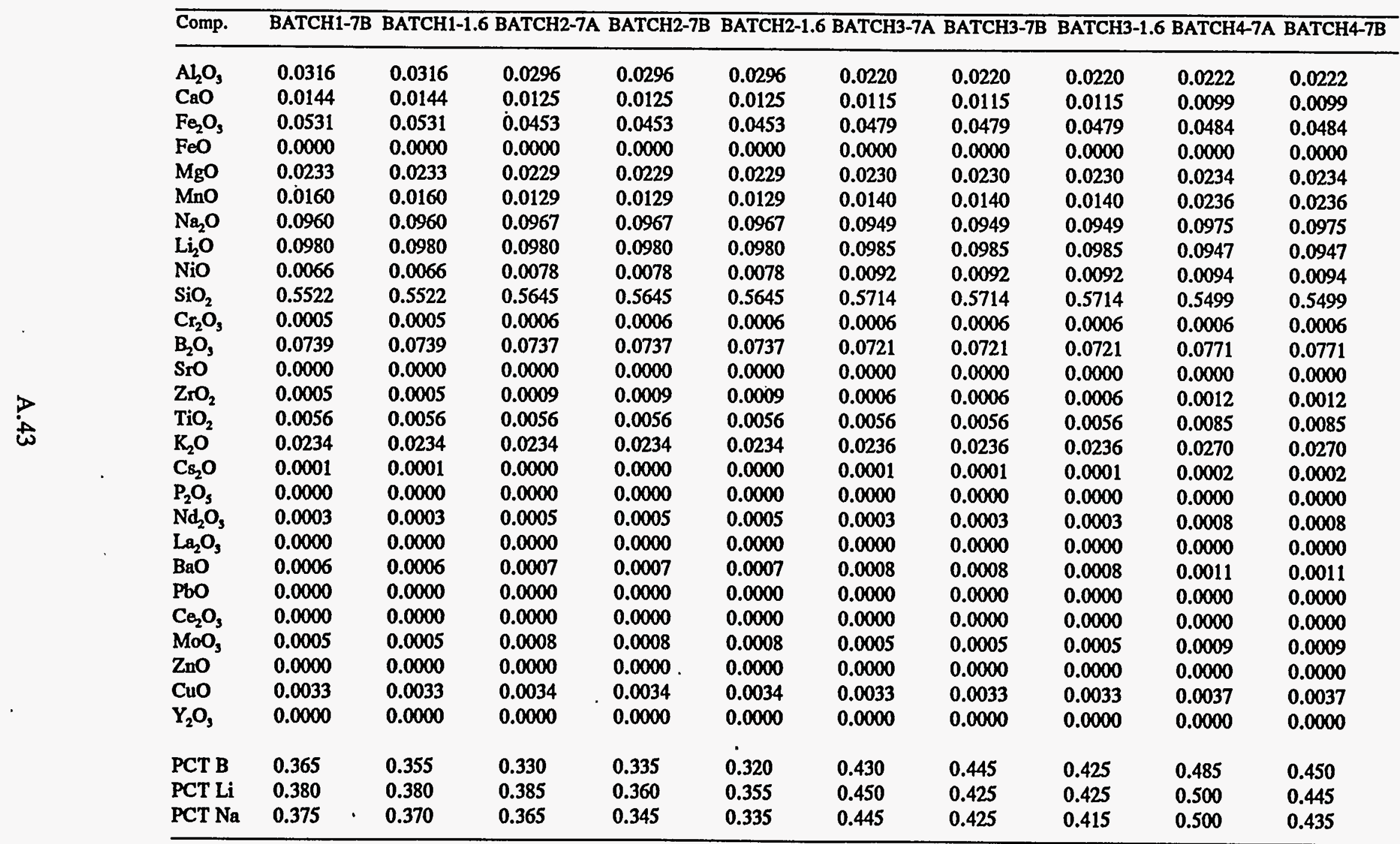

Note: Glass names are those in the reference from which the data were taken, except some names have been shortened. 
Table A.10. SRTC Homogeneous Glass Compositions (mole fractions) and PCT Normalized B, Li, and $\mathrm{Na}$ Releases $\left(\mathrm{g} / \mathrm{m}^{2}\right)$ (continued)

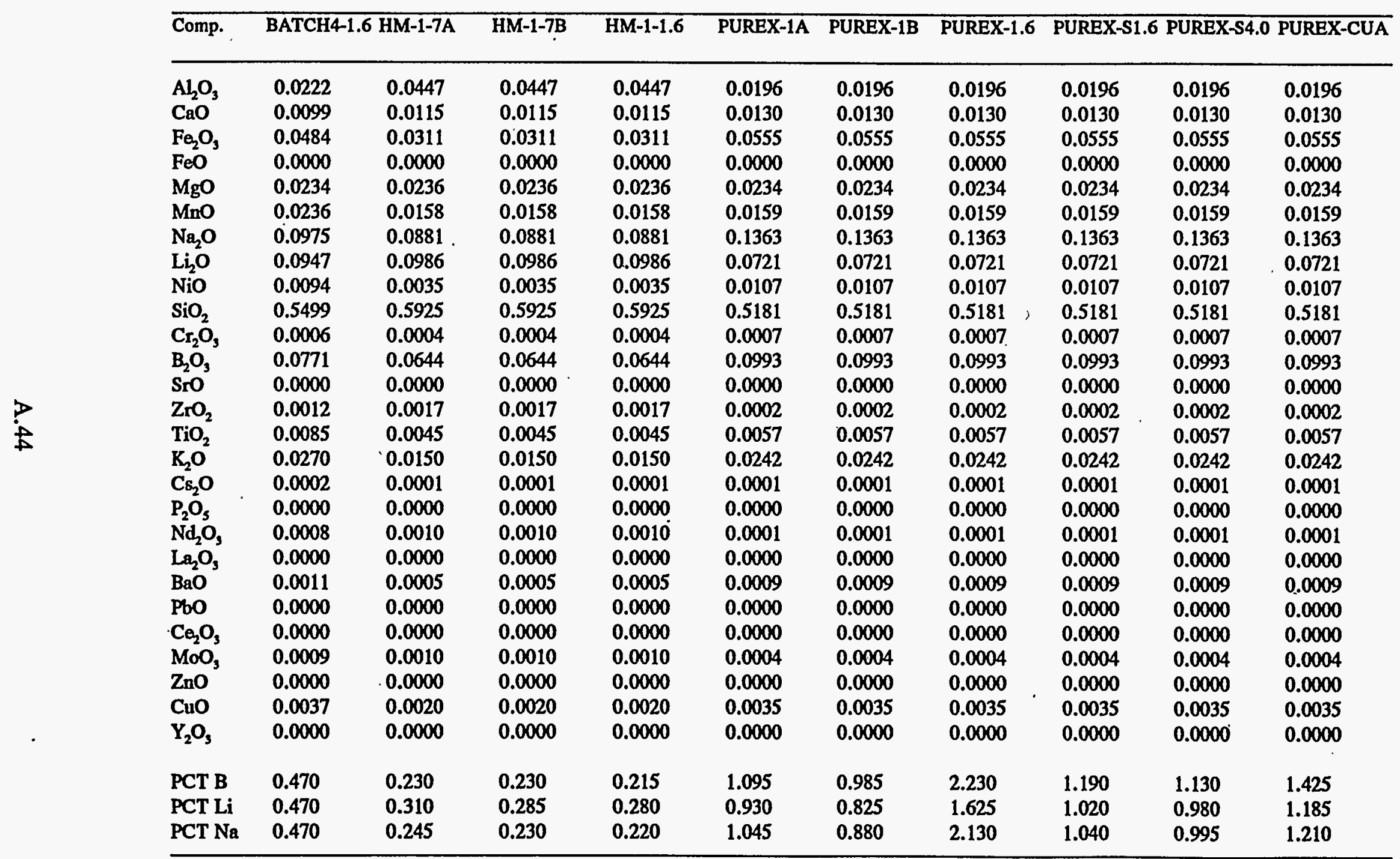

Note: Glass names are those in the reference from which the data were taken, except some names have been shortened. 
Table A.10. SRTC Homogeneous Glass Compositions (mole fractions) and PCT Normalized B, Li, and Na Releases $\left(\mathrm{g} / \mathrm{m}^{2}\right)$ (continued)

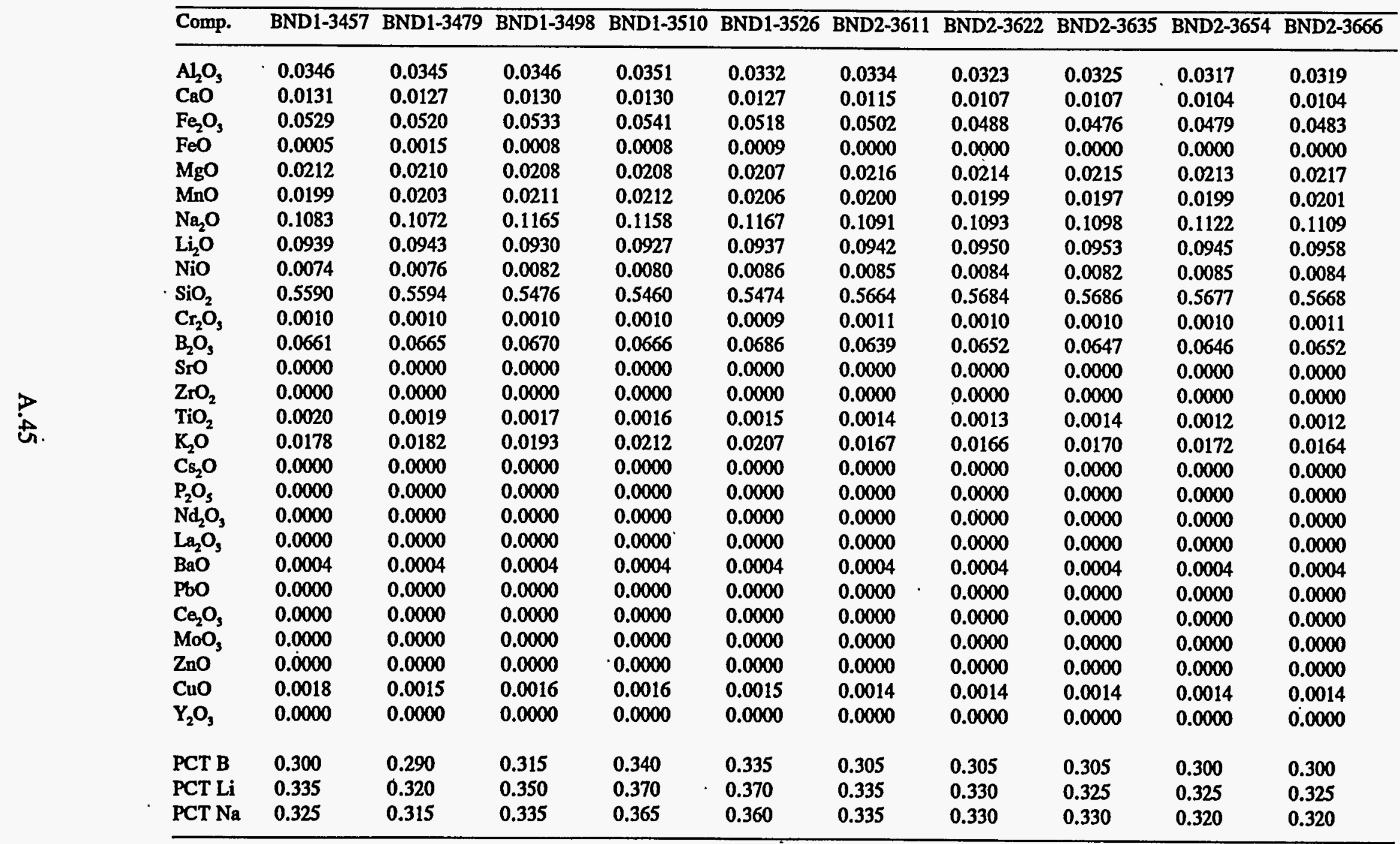

Note: Glass names are those in the reference from which the data were taken, except some names have been shortened. 


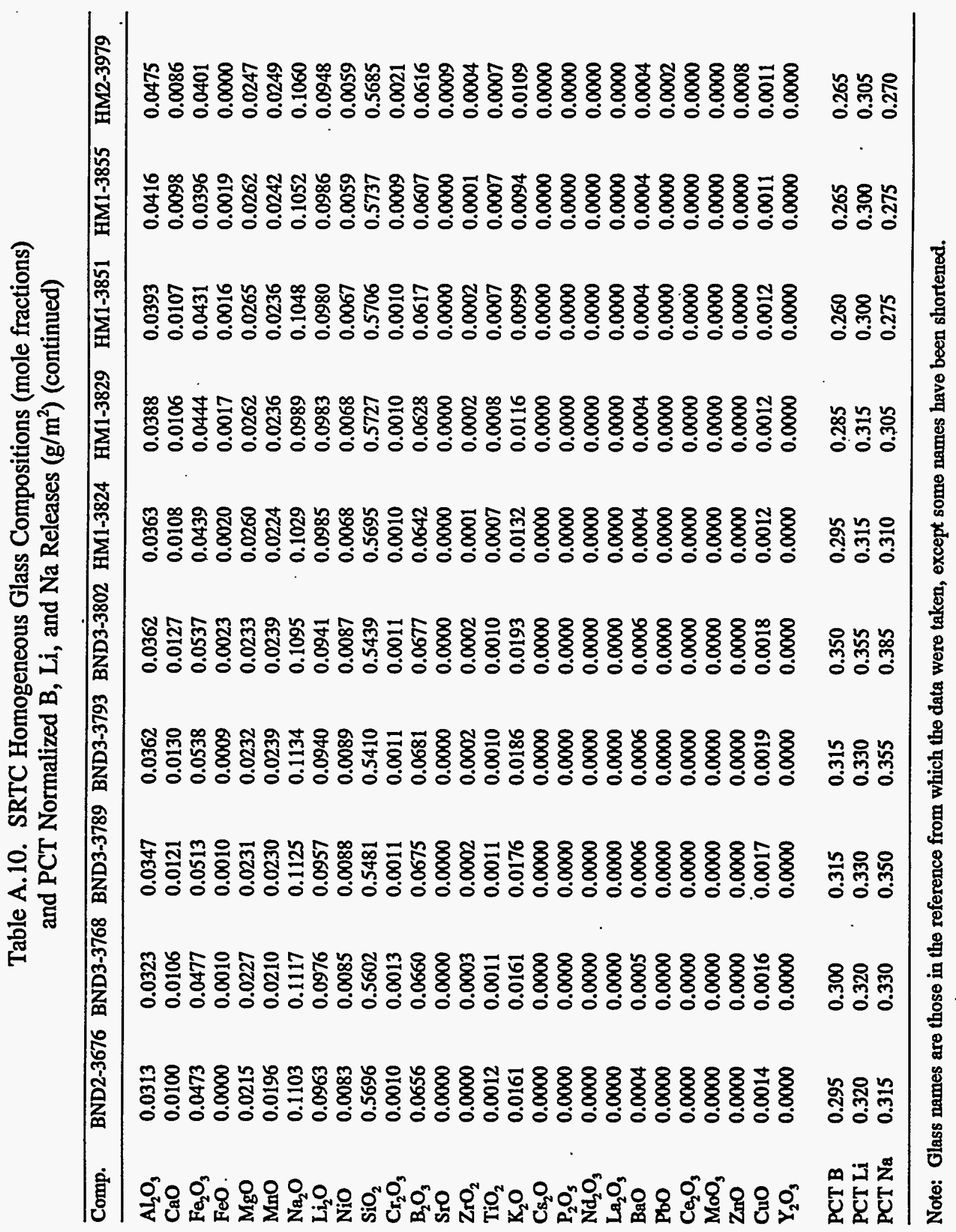


Table A.10. SRTC Homogeneous Glass Compositions (mole fractions)

and PCT Normalized B, Li, and Na Releases $\left(\mathrm{g} / \mathrm{m}^{2}\right)$ (continued)

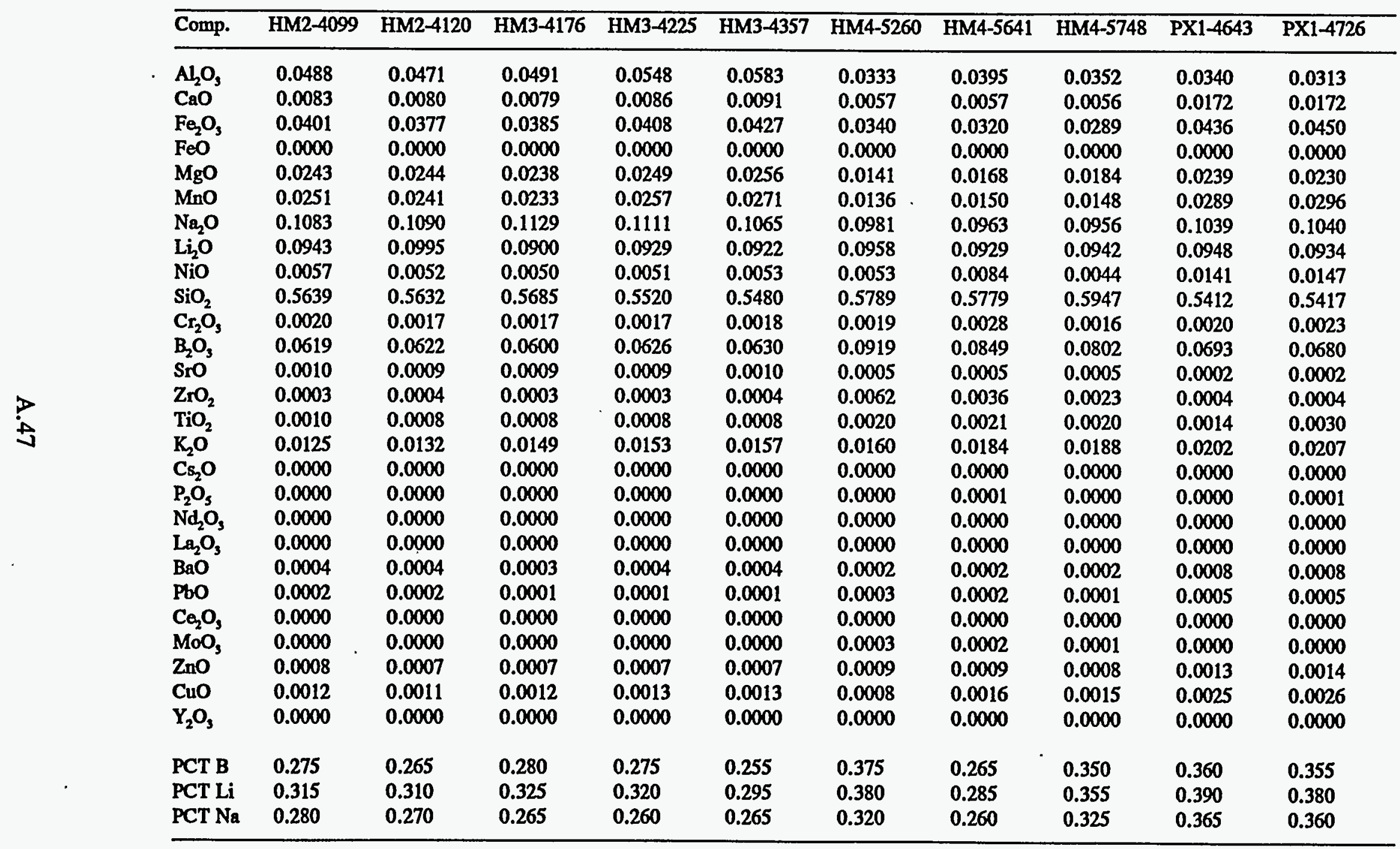

Note: Glass names are those in the reference from which the data were taken, except some names have been shortened. 
Table A.10. SRTC Homogeneous Glass Compositions (mole fractions) and PCT Normalized $\mathrm{B}, \mathrm{Li}$, and $\mathrm{Na}$ Releases $\left(\mathrm{g} / \mathrm{m}^{2}\right)$ (continued)

\begin{tabular}{|c|c|c|c|c|c|c|c|c|c|}
\hline Comp. & PX1-4776 & PX2-4455 & PX2-4509. & PX2-4566 & MG-9 & MG-18 & MG-25 & MG-28 & \\
\hline $\mathrm{Al}_{2} \mathrm{O}_{3}$ & 0.0274 & 0.0538 & 0.0446 & 0.0385 & 0.0012 & 0.0057 & 0.0876 & 0.1016 & \\
\hline $\mathrm{CaO}$ & 0.0152 & 0.0091 & 0.0133 & 0.0170 & 0.1001 & 0.1027 & 0.0370 & 0.0000 & \\
\hline $\mathrm{Fe}_{2} \mathrm{O}_{3}$ & 0.0402 & 0.0384 & 0.0450 & 0.0448 & 0.0000 & 0.0000 & 0.0355 & 0.0496 & . \\
\hline $\mathrm{FeO}$ & 0.0000 & 0.0000 & 0.0000 & 0.0000 & 0.0000 & 0.0000 & 0.0111 & 0.0212 & \\
\hline $\mathrm{MgO}$ & 0.0235 & 0.0254 & 0.0252 & 0.0245 & 0.0000 & 0.0000 & 0.0000 & 0.0000 & \\
\hline $\mathrm{MnO}$ & 0.0257 & 0.0257 & 0.0280 & 0.0300 & 0.0000 & 0.0000 & 0.0000 & 0.0000 & \\
\hline $\mathrm{Na}_{2} \mathrm{O}$ & 0.0993 & 0.1085 & 0.1007 & 0.1042 & 0.2543 & 0.2576 & 0.1720 & 0.1925 & \\
\hline $\mathrm{Li}_{2} \mathrm{O}$ & 0.0968 & 0.0934 & 0.0944 & 0.0943 & 0.0000 & 0.0000 & 0.0000 & 0.0000 & \\
\hline $\mathrm{NiO}$ & 0.0135 & 0.0055 & 0.0103 & 0.0133 & 0.0000 & 0.0000 & 0.0000 & 0.0000 & \\
\hline $\mathrm{SiO}_{2}$ & 0.5619 & 0.5558 & 0.5458 & 0.5335 & 0.5953 & 0.4912 & 0.5212 & 0.5412 & \\
\hline $\mathrm{Cr}_{2} \mathrm{O}_{3}$ & 0.0022 & 0.0017 & 0.0020 & 0.0020 & 0.0000 & 0.0000 & 0.0000 & 0.0000 & \\
\hline $\mathrm{B}_{2} \mathrm{O}_{3}$ & 0.0671 & 0.0635 & 0.0675 & 0.0698 & 0.0491 & 0.1428 & 0.1357 & 0.0939 & \\
\hline SrO & 0.0001 & 0.0009 & 0.0005 & 0.0003 & 0.0000 & 0.0000 & 0.0000 & 0.0000 & \\
\hline $\mathrm{ZrO}_{2}$ & 0.0004 & 0.0003 & 0.0003 & 0.0004 & 0.0000 & 0.0000 & 0.0000 & 0.0000 & \\
\hline $\mathrm{TiO}_{2}$ & 0.0029 & 0.0008 & 0.0014 & 0.0018 & 0.0000 & 0.0000 & 0.0000 & 0.0000 & \\
\hline $\mathrm{K}_{2} \mathrm{O}$ & 0.0189 & 0.0147 & 0.0172 & 0.0210 & 0.0000 & 0.0000 & 0.0000 & 0.0000 & \\
\hline $\mathrm{Cs}_{2} \mathrm{O}$ & 0.0000 & 0.0000 & 0.0000 & 0.0000 & 0.0000 & 0.0000 & 0.0000 & 0.0000 & \\
\hline $\mathrm{P}_{2} \mathrm{O}_{5}$ & 0.0000 & 0.0000 & 0.0000 & 0.0000 & 0.0000 & 0.0000 & 0.0000 & 0.0000 & \\
\hline $\mathrm{Nd}_{2} \mathrm{O}_{3}$ & 0.0000 & 0.0000 & 0.0000 & 0.0000 & 0.0000 & 0.0000 & 0.0000 & 0.0000 & \\
\hline $\mathrm{La}_{2} \mathrm{O}_{3}$ & 0.0000 & 0.0000 & 0.0000 & 0.0000 & 0.0000 & 0.0000 & 0.0000 & 0.0000 & \\
\hline $\mathrm{BaO}$ & 0.0007 & 0.0004 & 0.0006 & 0.0008 & 0.0000 & 0.0000 & 0.0000 & 0.0000 & . \\
\hline $\mathrm{PbO}$ & 0.0005 & 0.0001 & 0.0003 & 0.0004 & 0.0000 & 0.0000 & 0.0000 & 0.0000 & \\
\hline $\mathrm{Ce}_{2} \mathrm{O}_{3}$ & 0.0000 & 0.0000 & 0.0000 & 0.0000 & 0.0000 & 0.0000 & 0.0000 & 0.0000 & \\
\hline $\mathrm{MoO}_{3}$ & 0.0000 & 0.0000 & 0.0000 & 0.0000 & 0.0000 & 0.0000 & 0.0000 & 0.0000 & \\
\hline $\mathrm{ZnO}$ & 0.0013 & 0.0008 & 0.0011 & 0.0012 & 0.0000 & 0.0000 & 0.0000 & 0.0000 & \\
\hline $\mathrm{CuO}$ & 0.0023 & 0.0013 & 0.0019 & 0.0023 & 0.0000 & 0.0000 & 0.0000 & 0.0000 & \\
\hline $\mathrm{Y}_{2} \mathrm{O}_{3}$ & 0.0000 & 0.0000 & 0.0000 & 0.0000 & 0.0000 & 0.0000 & 0.0000 & 0.0000 & \\
\hline PCT B & 0.395 & 0.240 & 0.290 & 0.365 & 35.120 & 28.910 & 0.415 & 0.315 & \\
\hline PCT Li & 0.415 & 0.285 & 0.325 & 0.390 & • & • & • & • & \\
\hline $\mathrm{PCT} \mathbf{N a}$ & 0.385 & 0.240 & 0.305 & 0.365 & 28.210 & 23.885 & 0.395 & 0.360 & \\
\hline
\end{tabular}

Note: Glass names are those in the reference from which the data were taken, except some names have been shortened. 


\section{Appendix B}

\section{Scatterplot Matrices}

This appendix contains the scatterplot matrix for each data set described in Section 4. A scatterplot matrix in this appendix consists of a matrix of scatterplots of pairs of glass components for a given data set. Because individual plots in a scatterplot matrix get quite small for larger numbers of components, only those components included in the first-order mixture model for each data set are included in the scatterplot matrix.

Various features of a data set can adversely affect property models fitted to the data set. Such features that can be detected in a scatterplot are:

(1) A component being constant or nearly constant over a data set. This indicates that it is impossible to accurately estimate the component's coefficient in empirical mixture models.

(2) A correlation between pairs of components in a scatterplot, indicated by the points having a linear appearance. The stronger such a correlation, the more difficult (and eventually impossible) it is to separate the effects of the components on glass properties using the data set. Incorrect empirical model coefficients can result if pairwise component correlations are strong enough.

(3) Clustering of points or an uneven coverage of pairwise combinations of components.

Clustering of points can indicate that the data set contains points with similar combinations of components in many compositions. An uneven coverage of pairwise combinations can indicate a lack of compositions in parts of the model domain. Both of these conditions can result in a poor basis for estimating the effects of the affected components on glass properties. However, an uneven coverage can be indicative of constraints imposed in collecting the data (e.g., avoiding compositions with low $\mathrm{SiO}_{2}$ and low $\mathrm{Al}_{2} \mathrm{O}_{3}$ ).

The presence or absence of any such bothersome features is discussed for each data set in the corresponding subsection of Section 4 . 

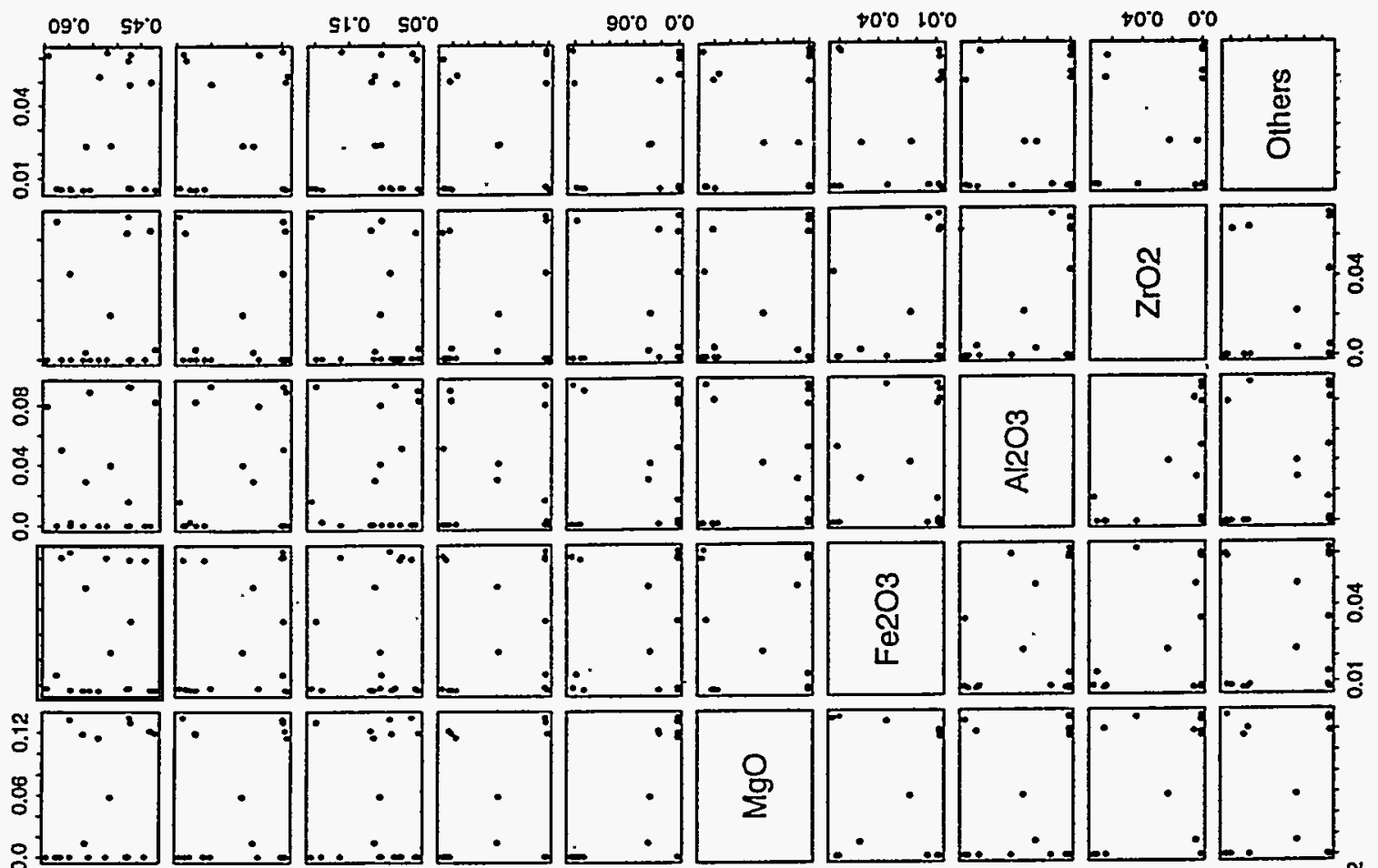

号

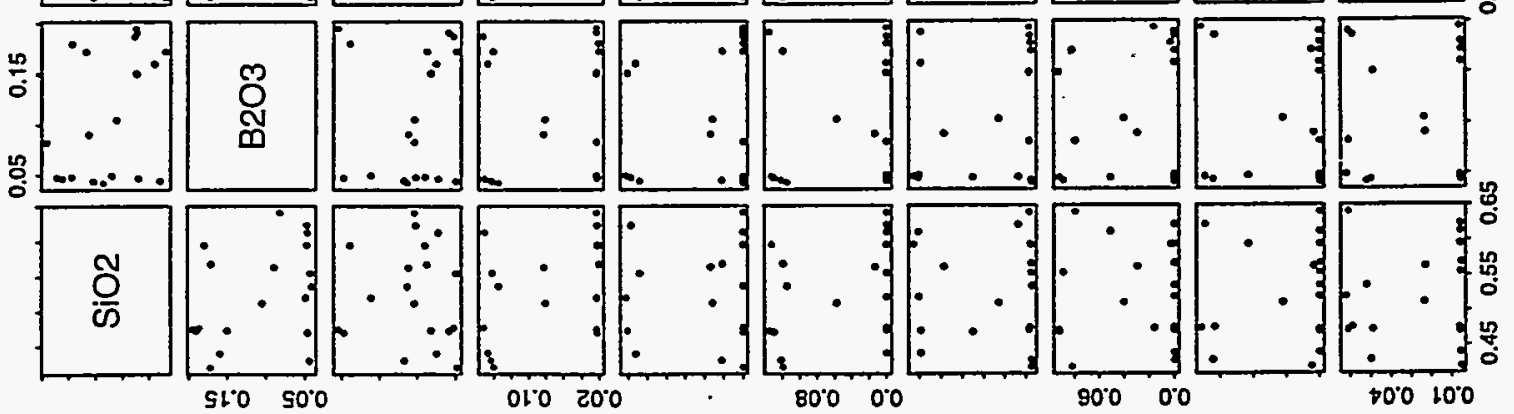



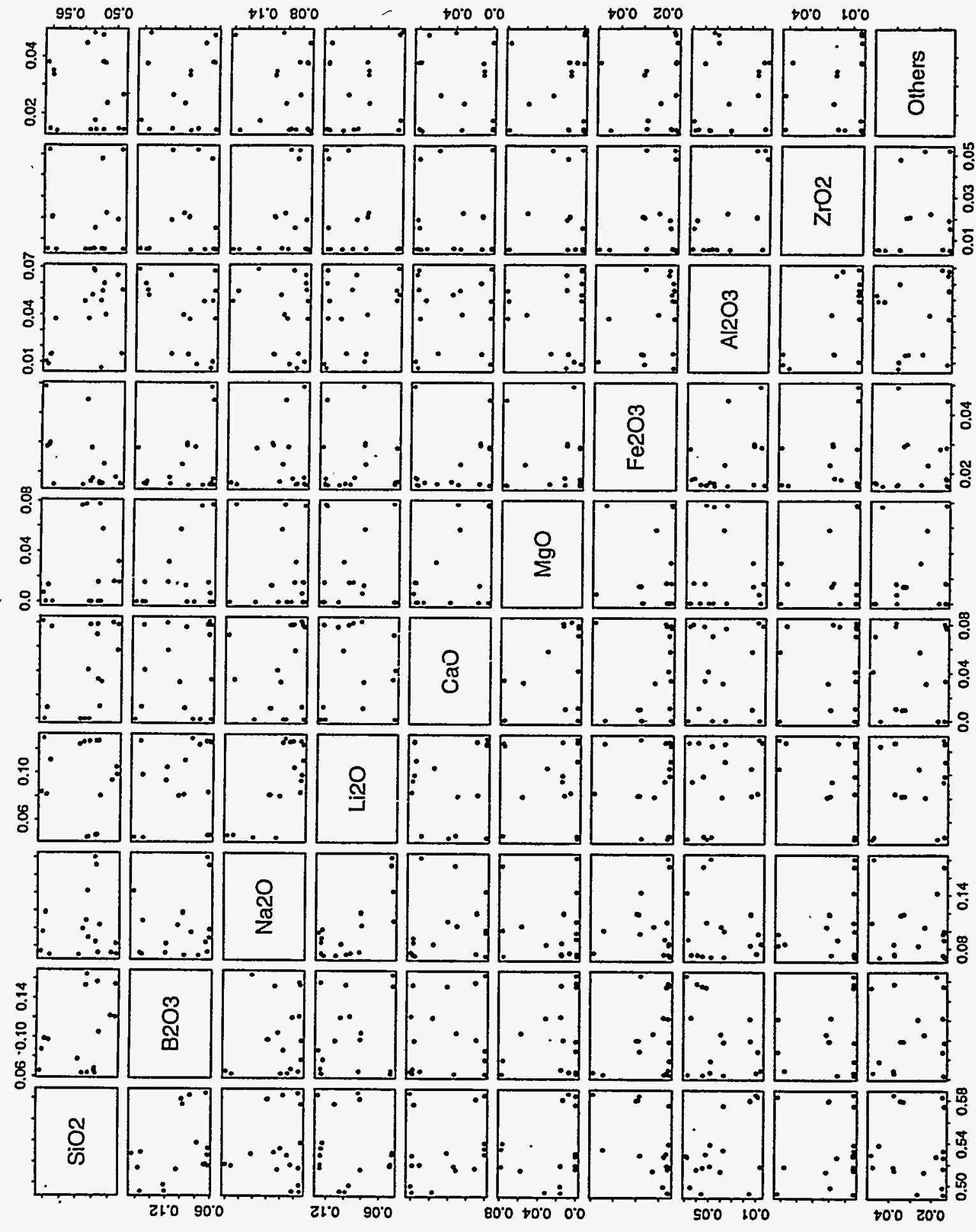

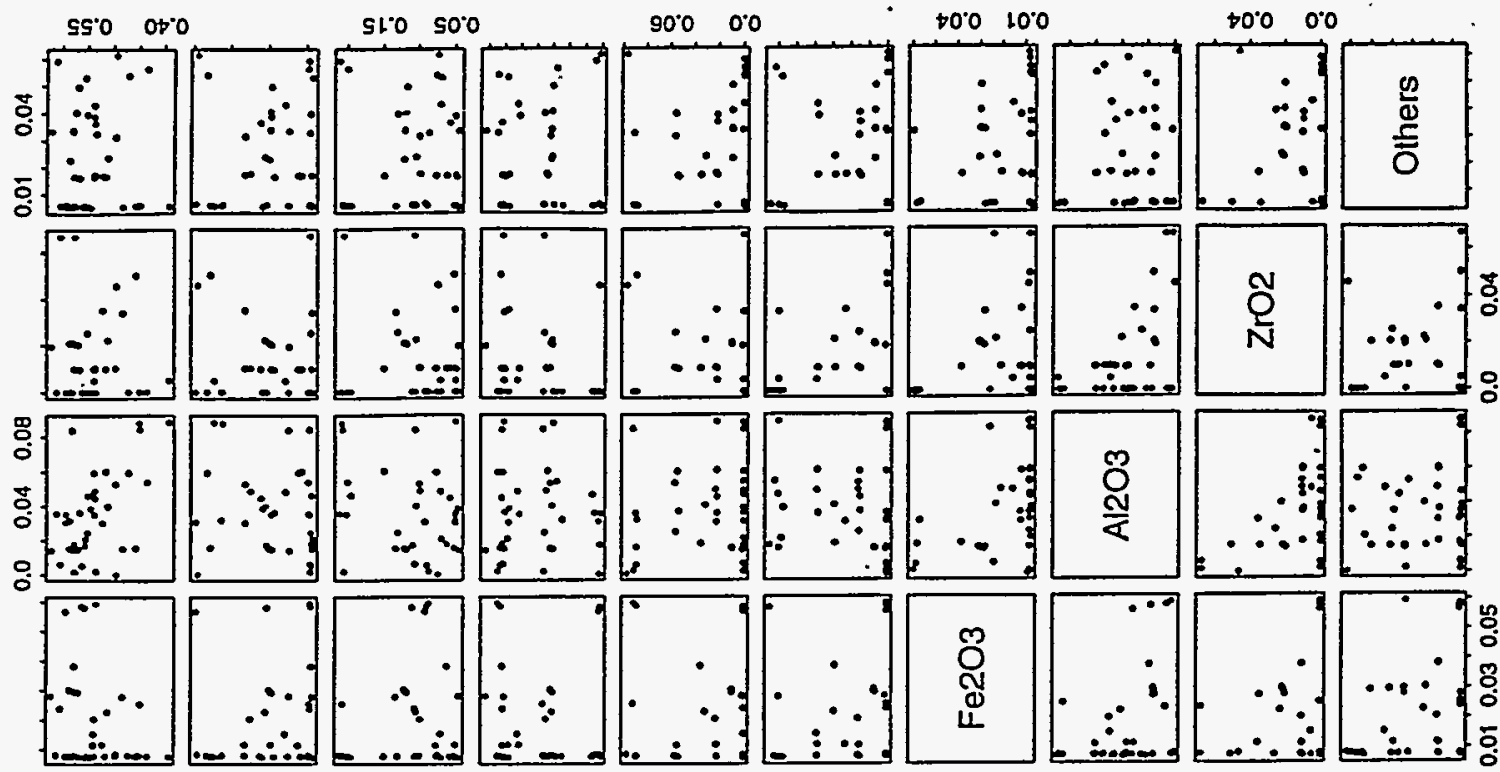

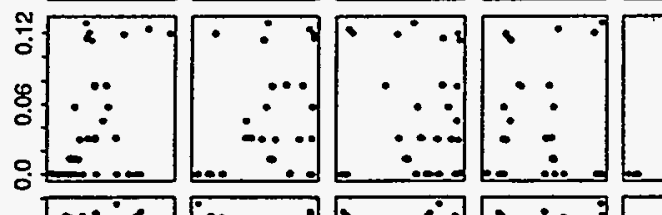
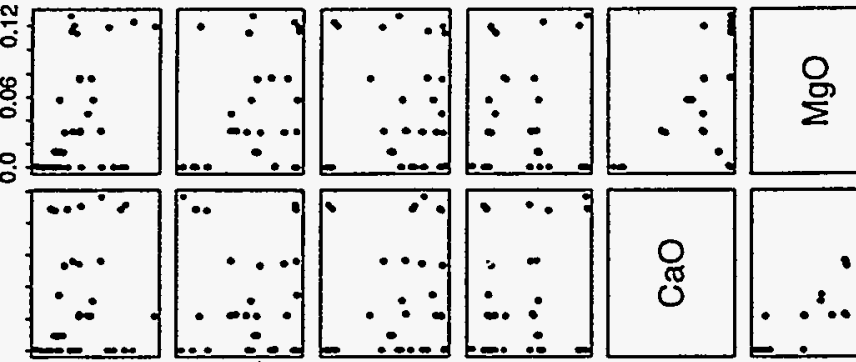

ญ్
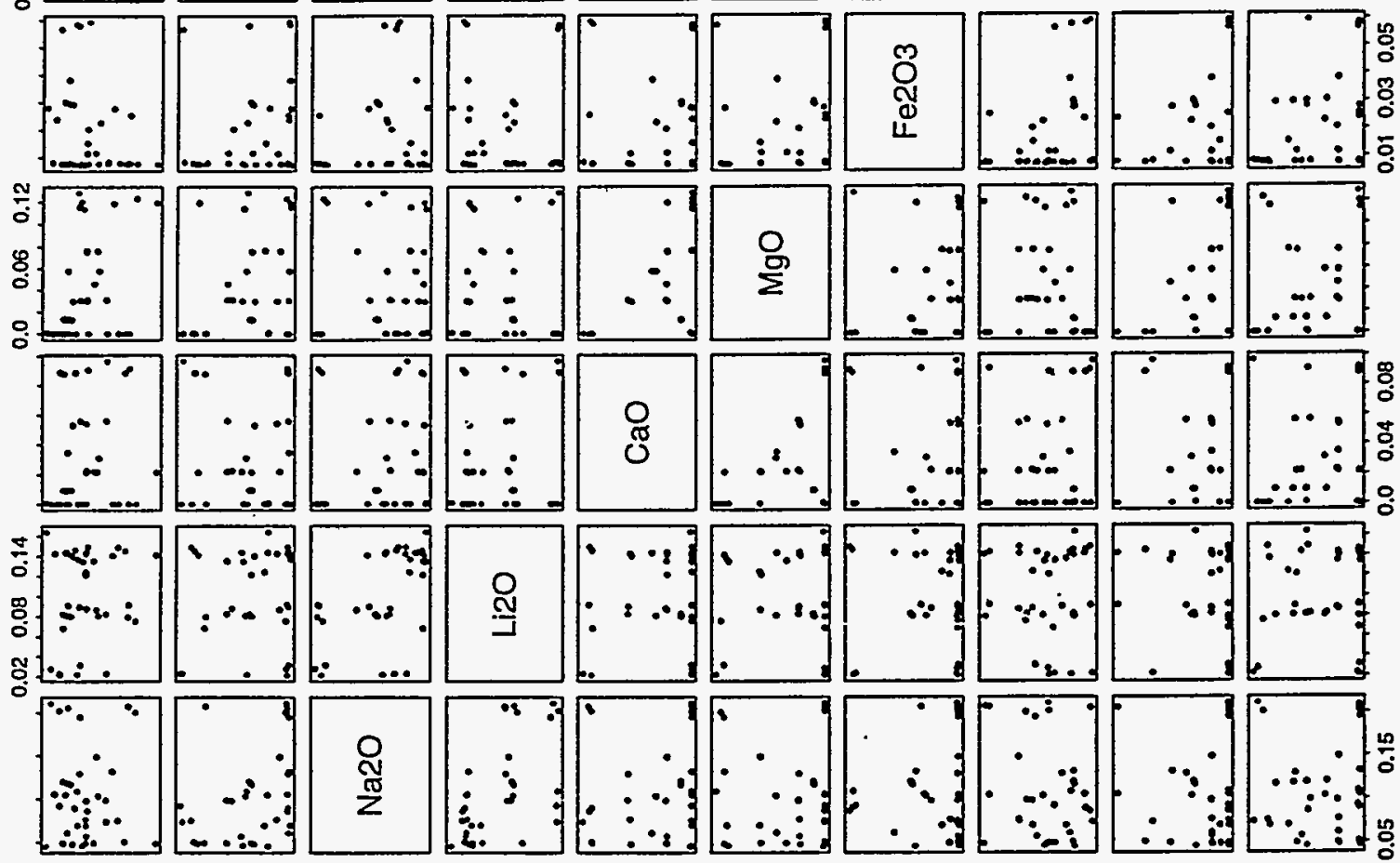

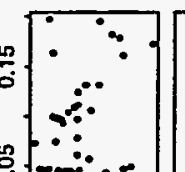

요
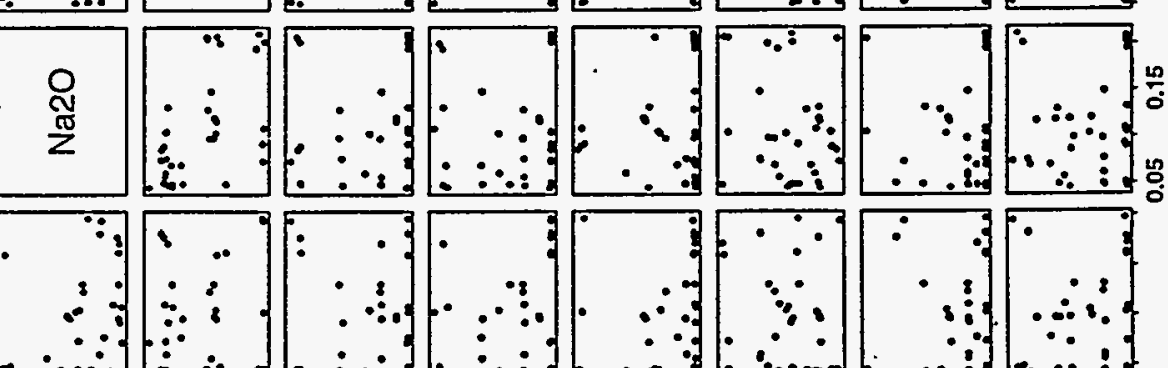

- $\frac{10}{0}$

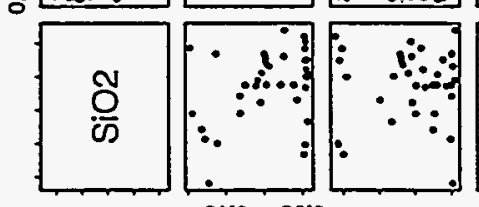

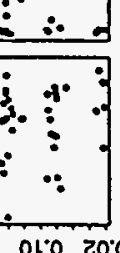
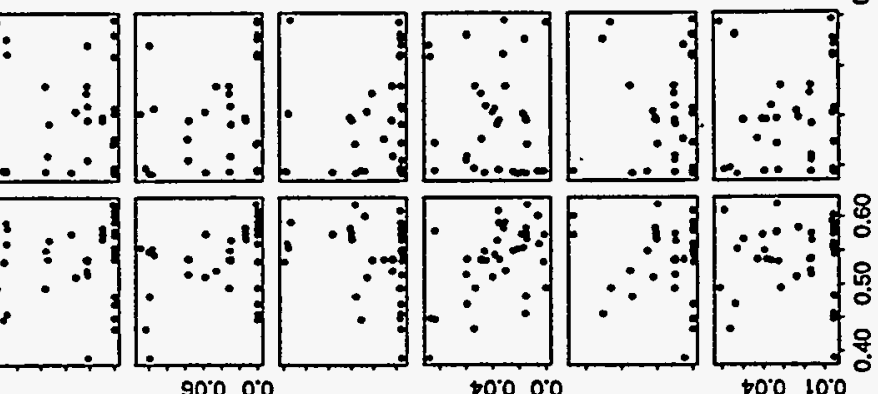

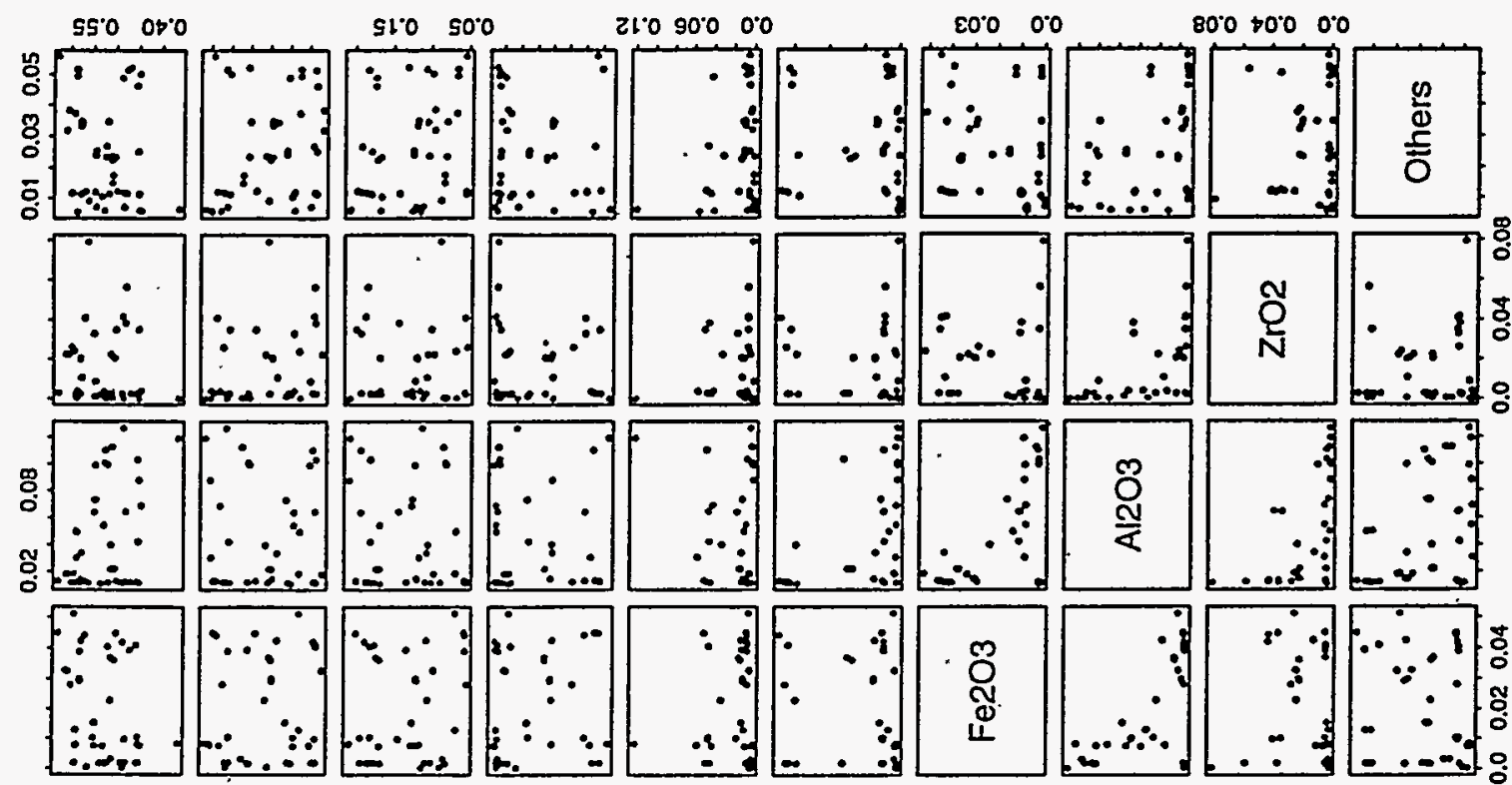

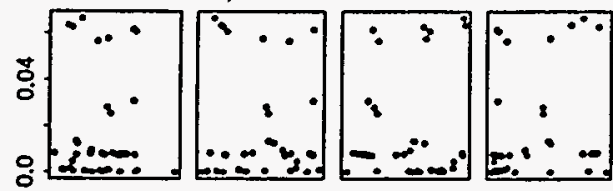
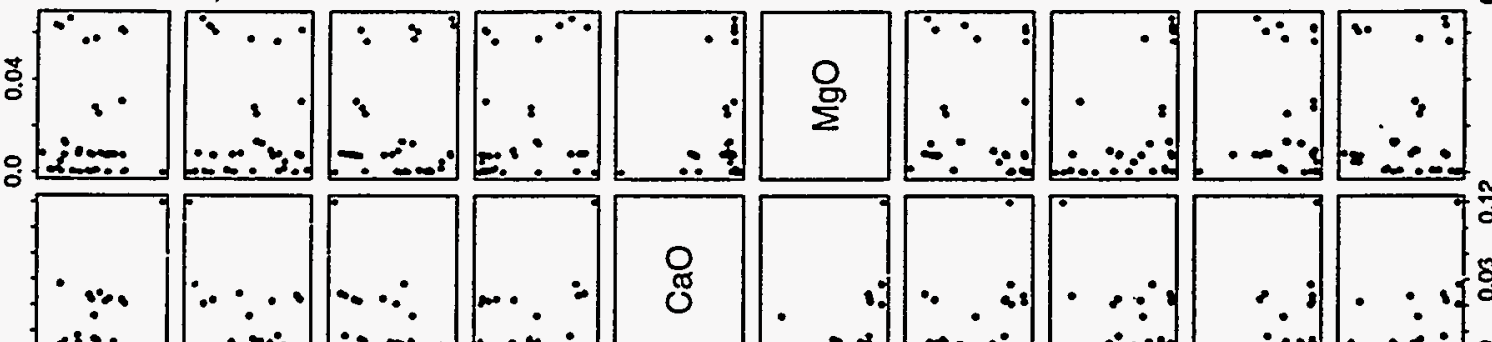

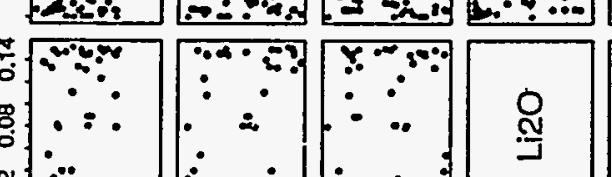

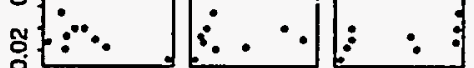
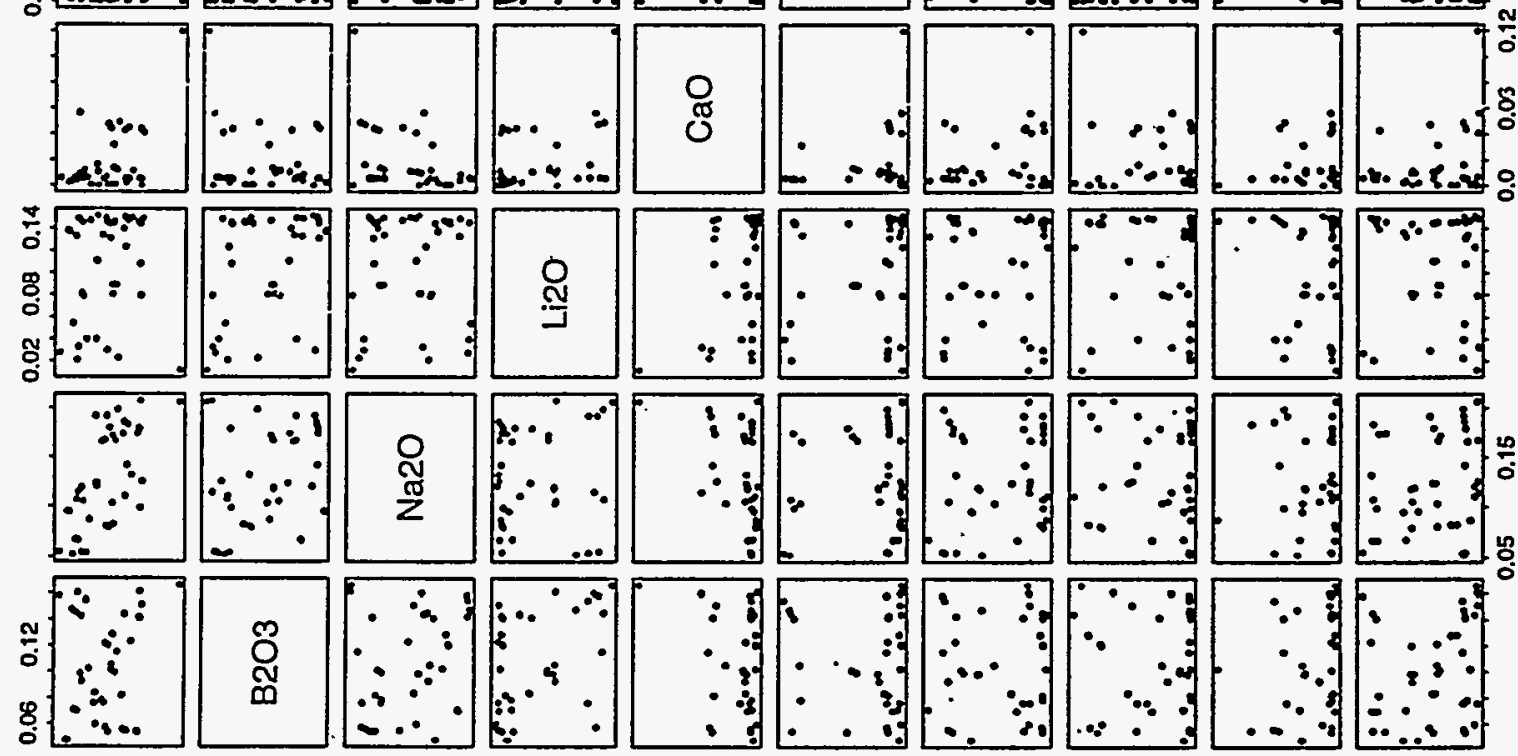

1.
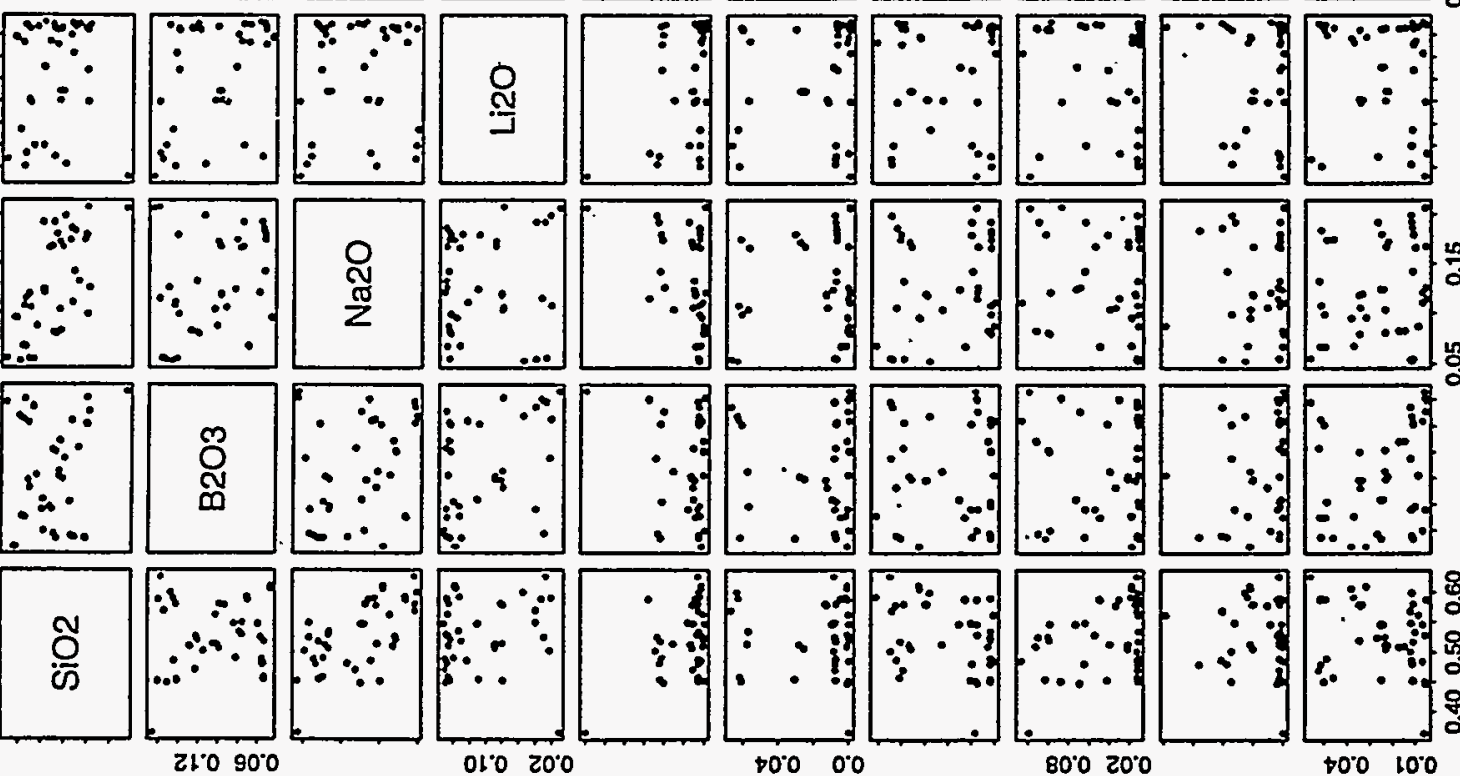

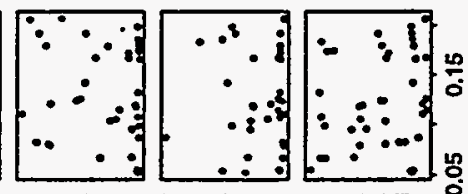
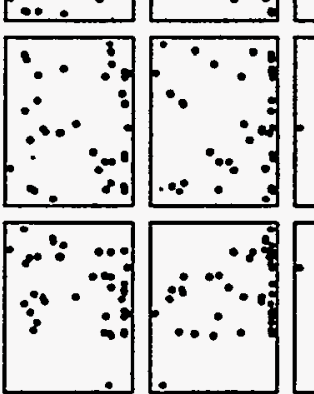

$80^{\circ} 020^{\circ} 0$

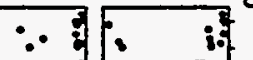

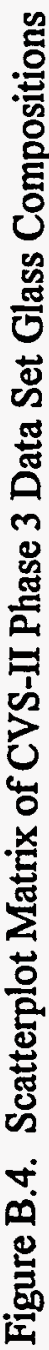




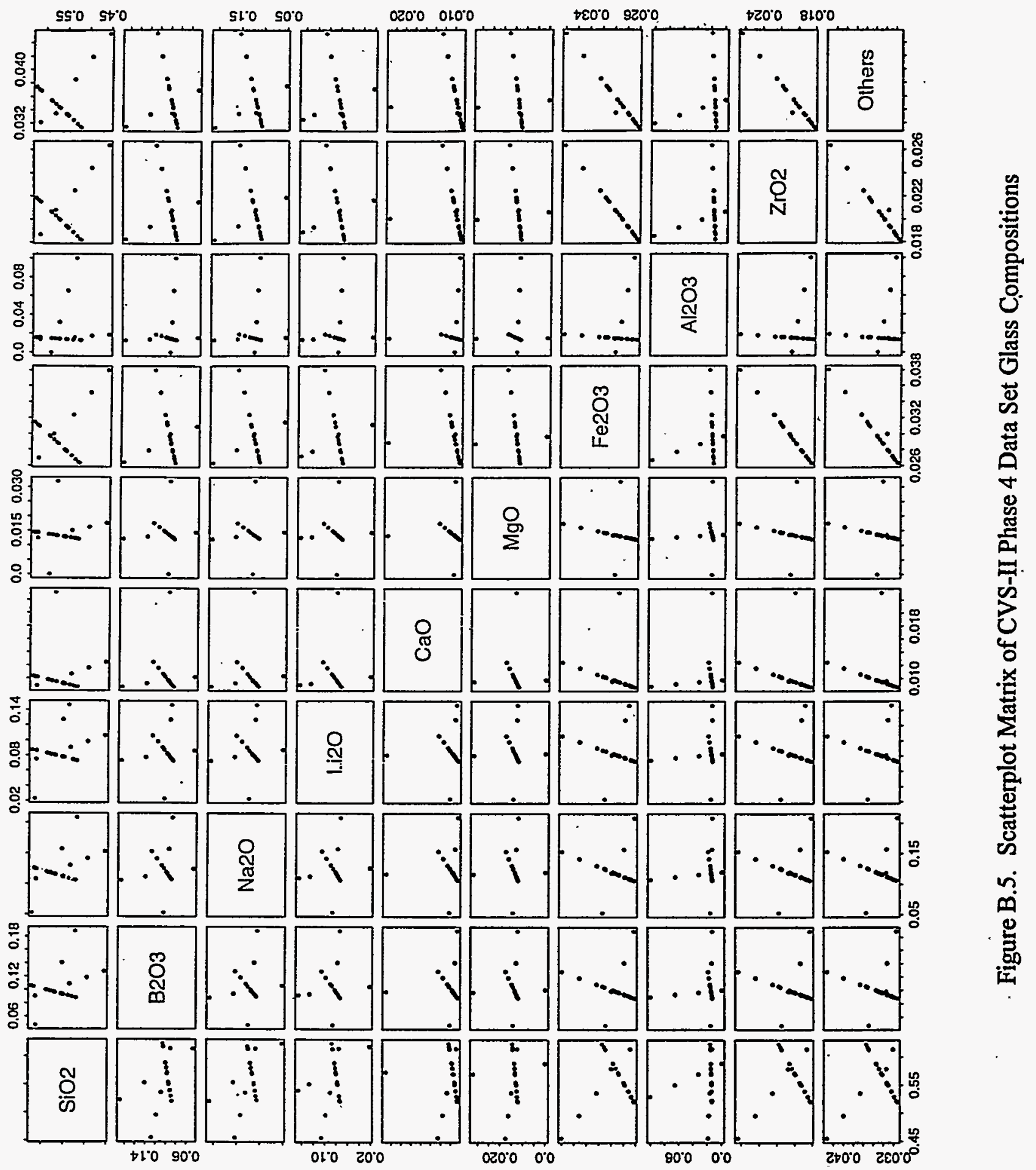




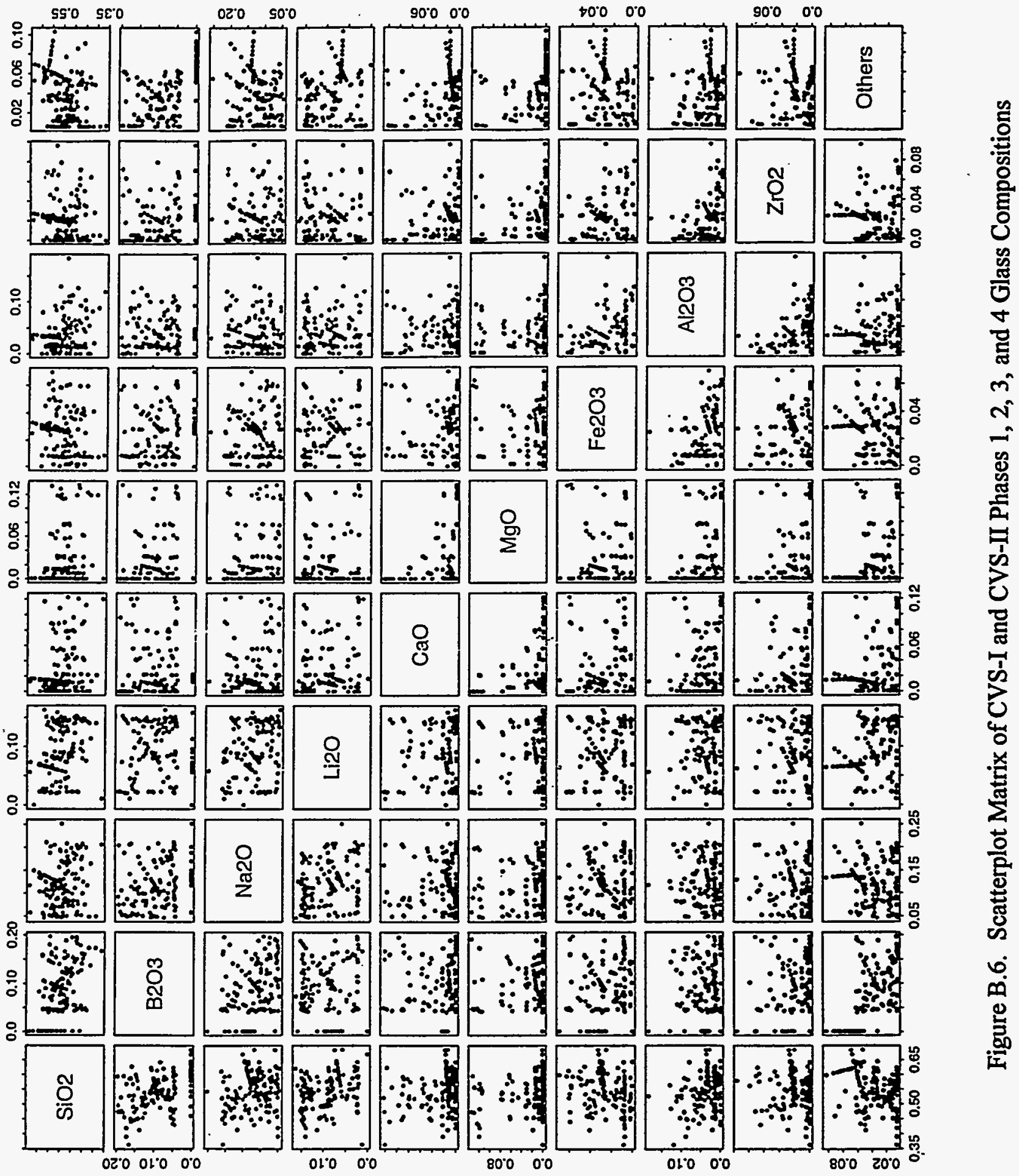

B. 7 


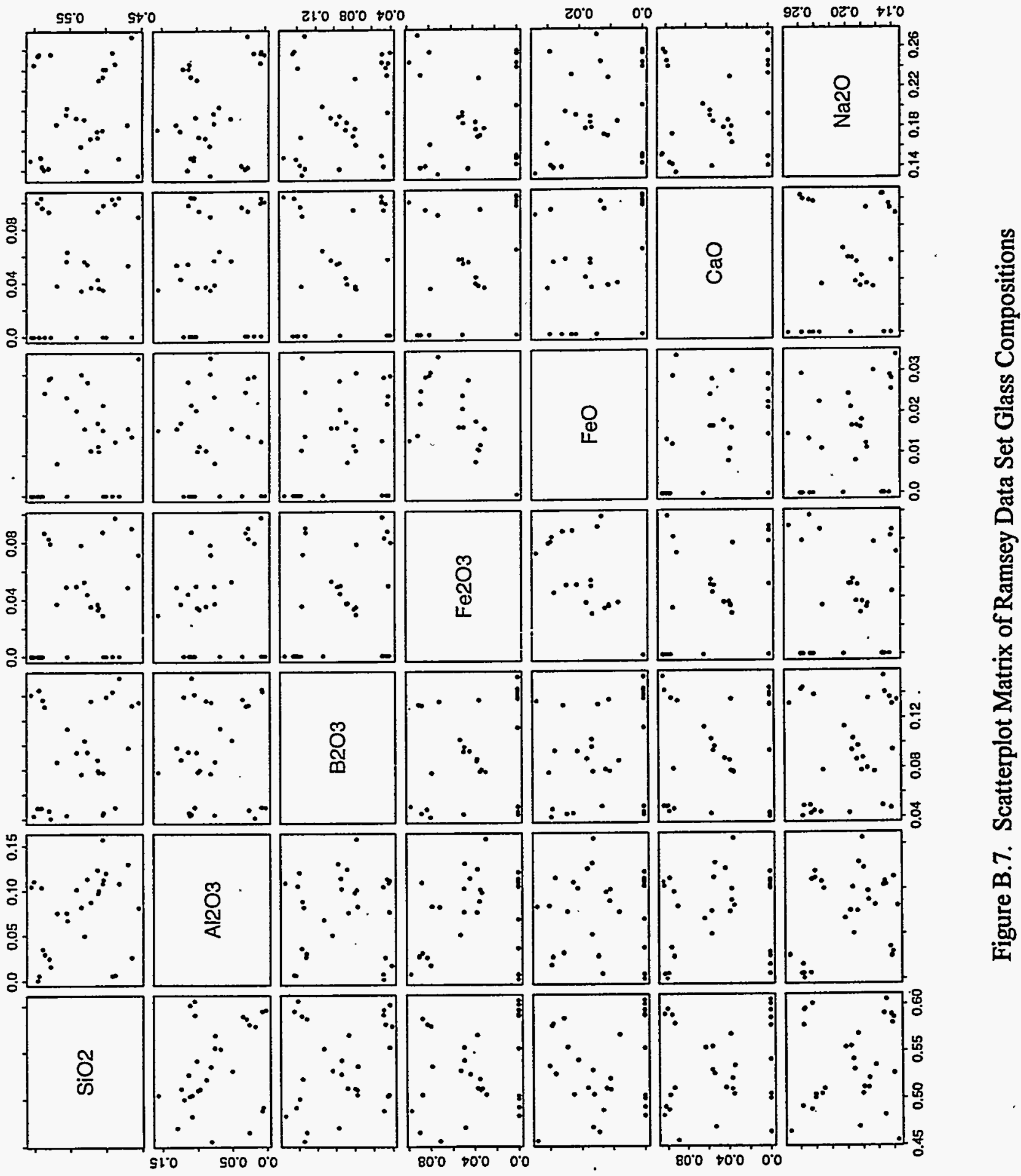




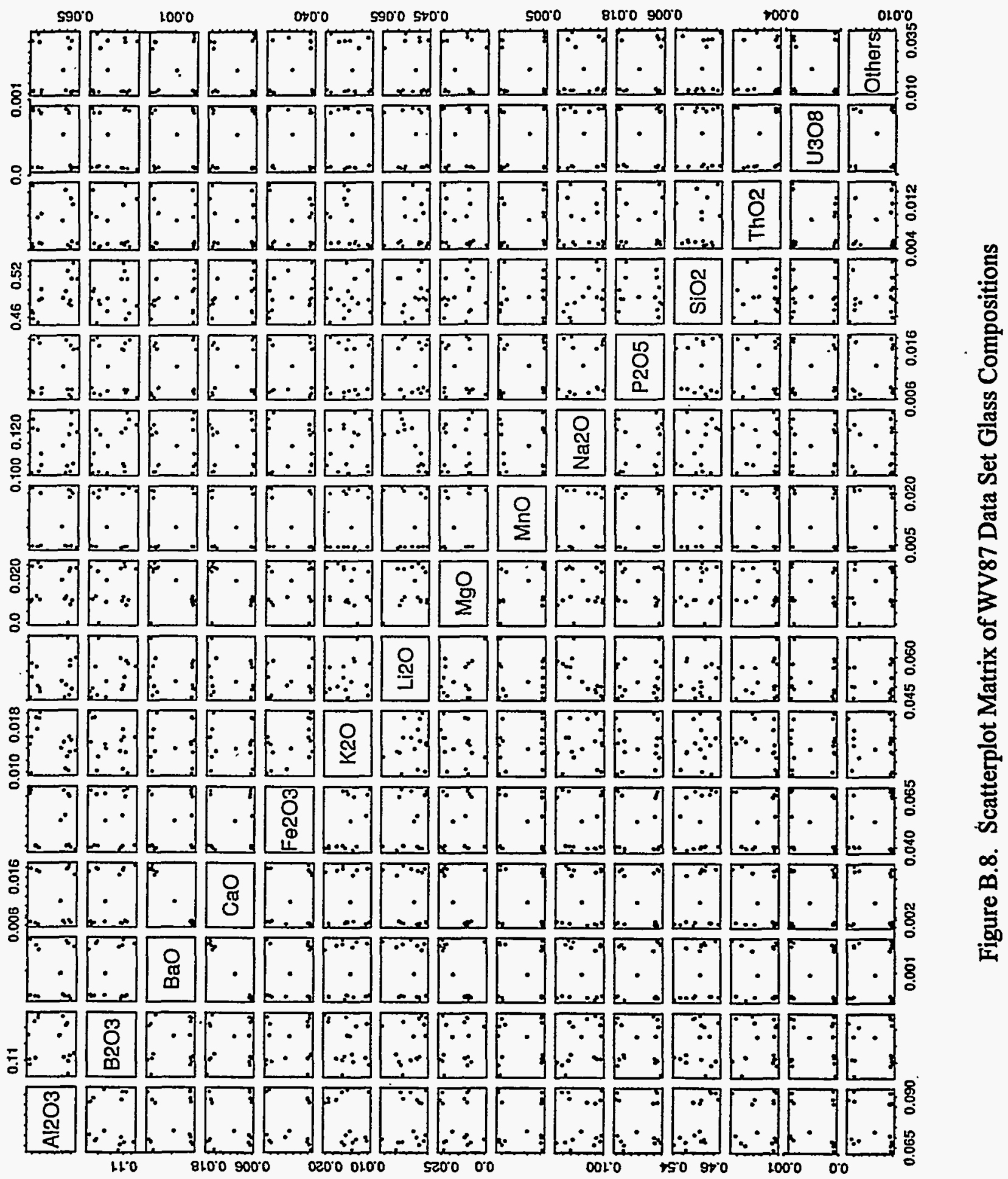



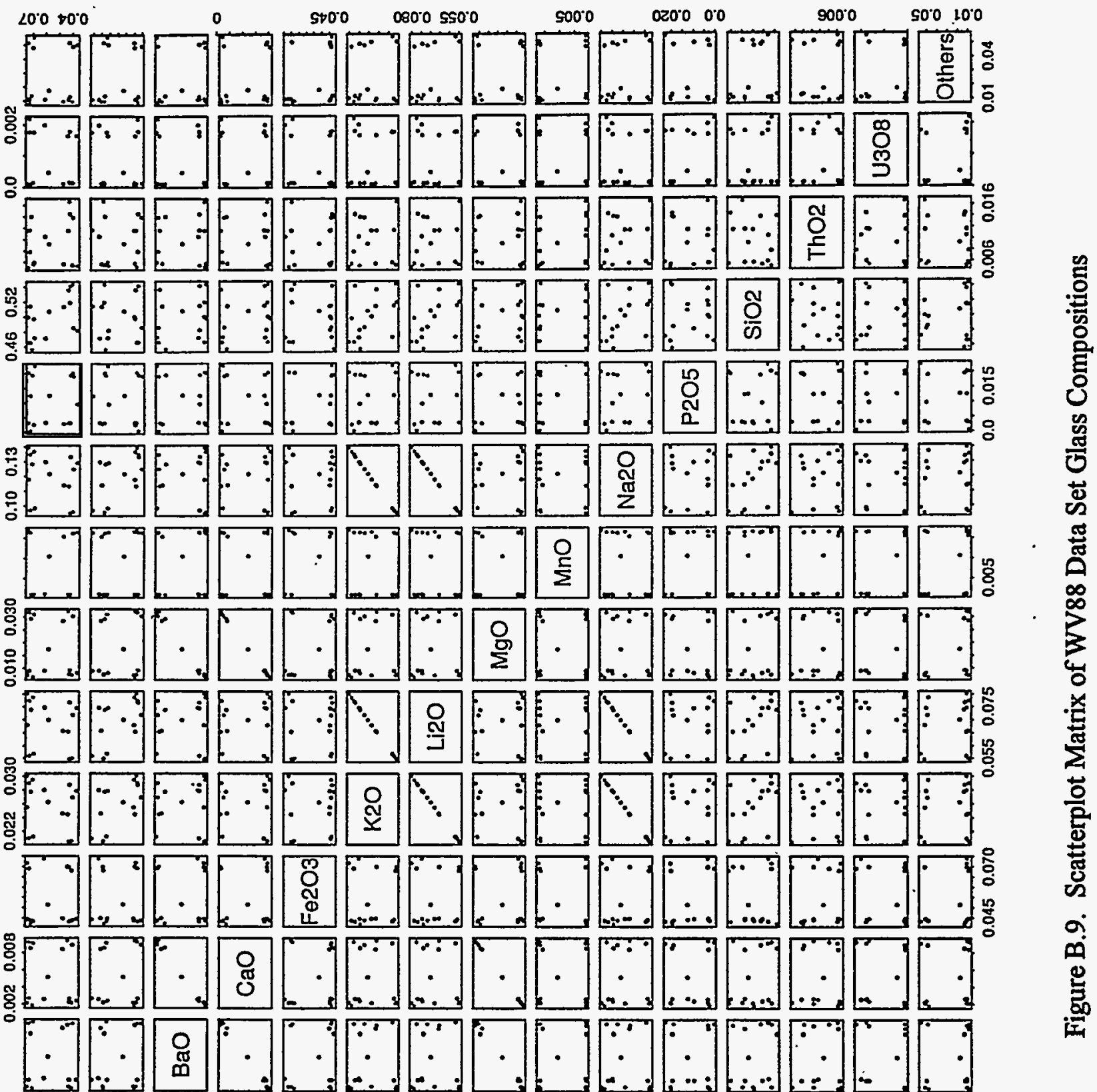

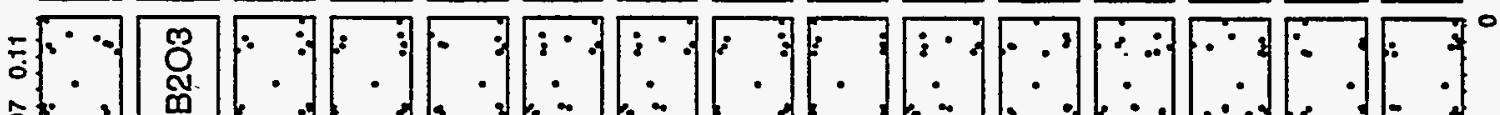

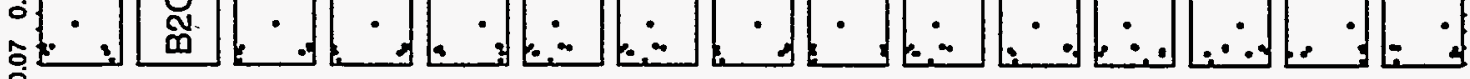

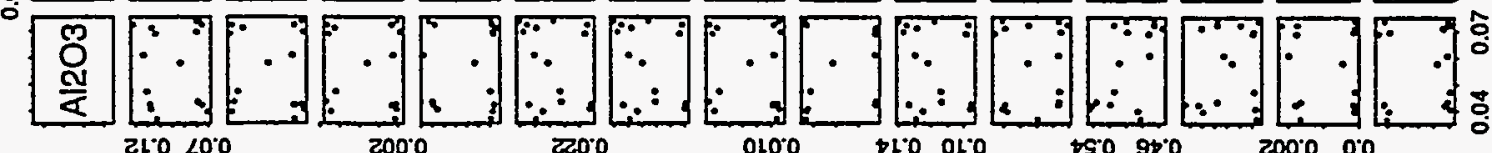



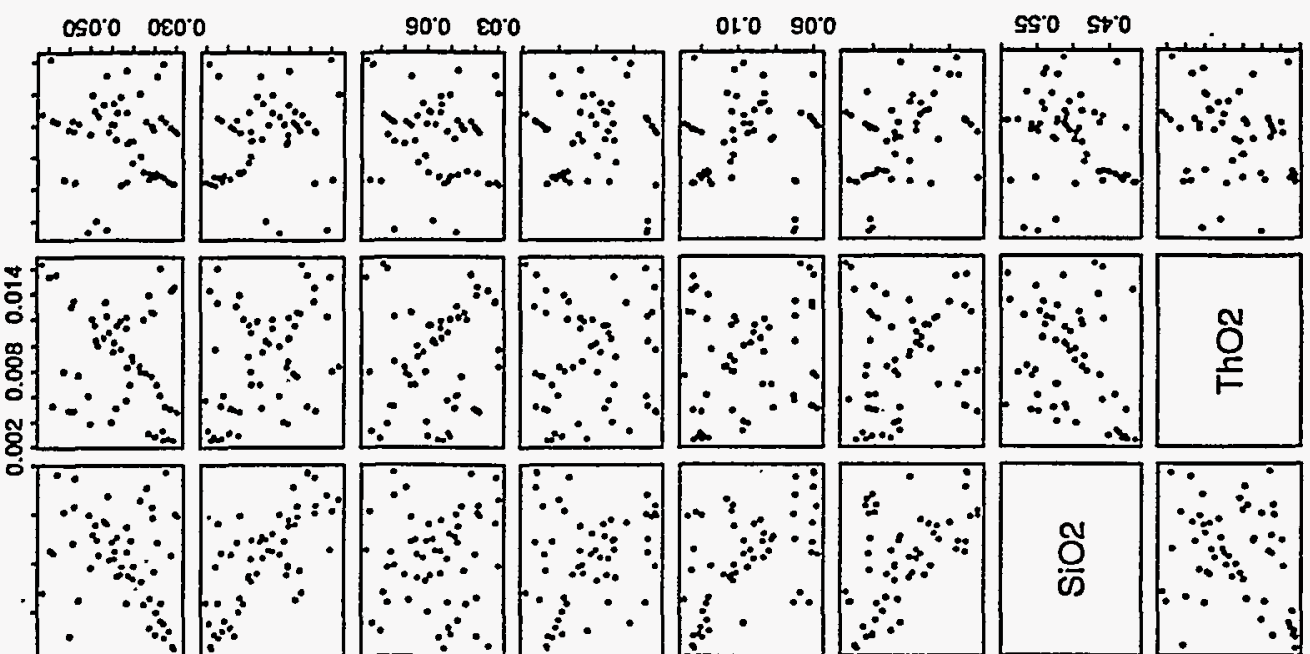

$800^{\circ} 0200^{\circ} 0$
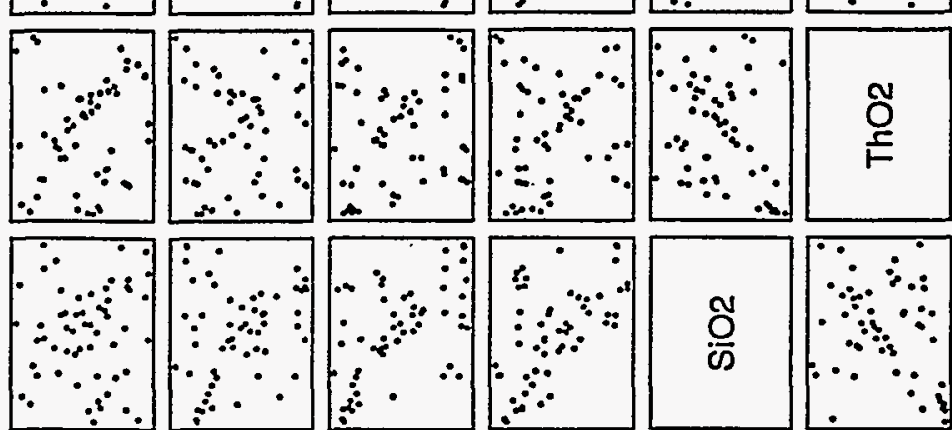

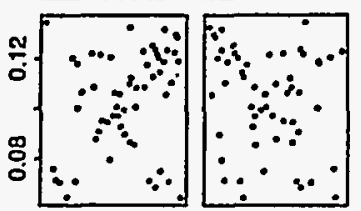
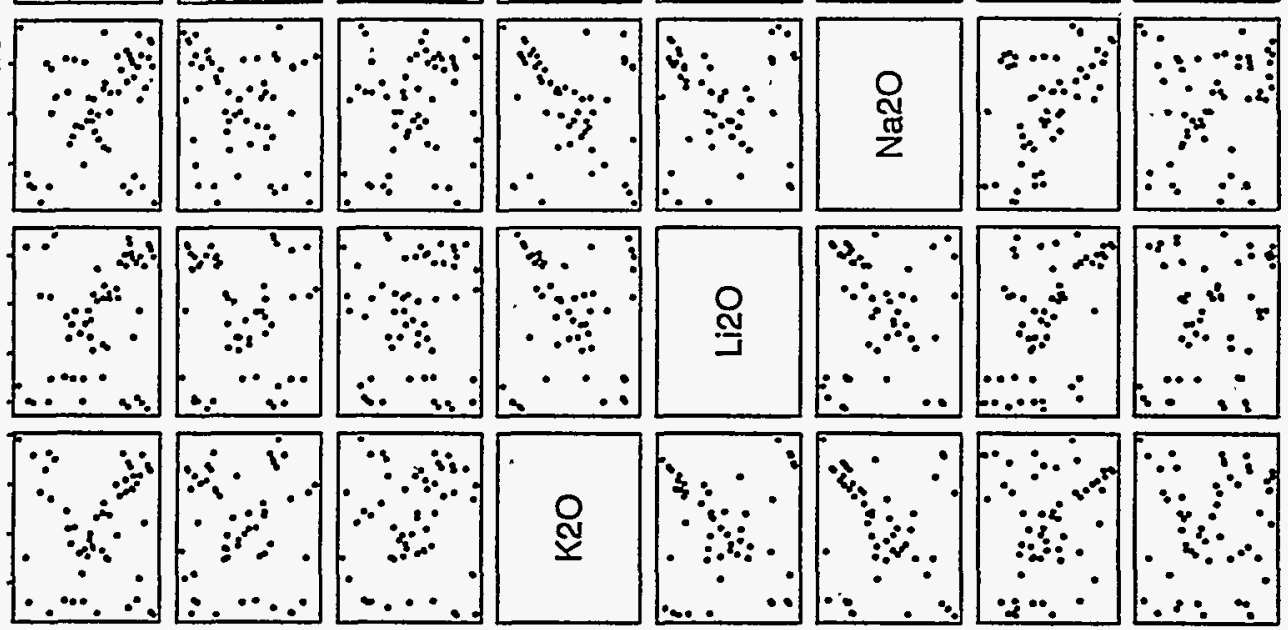

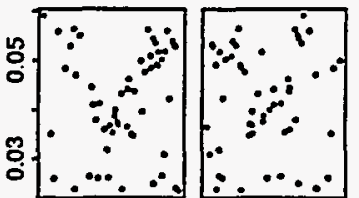
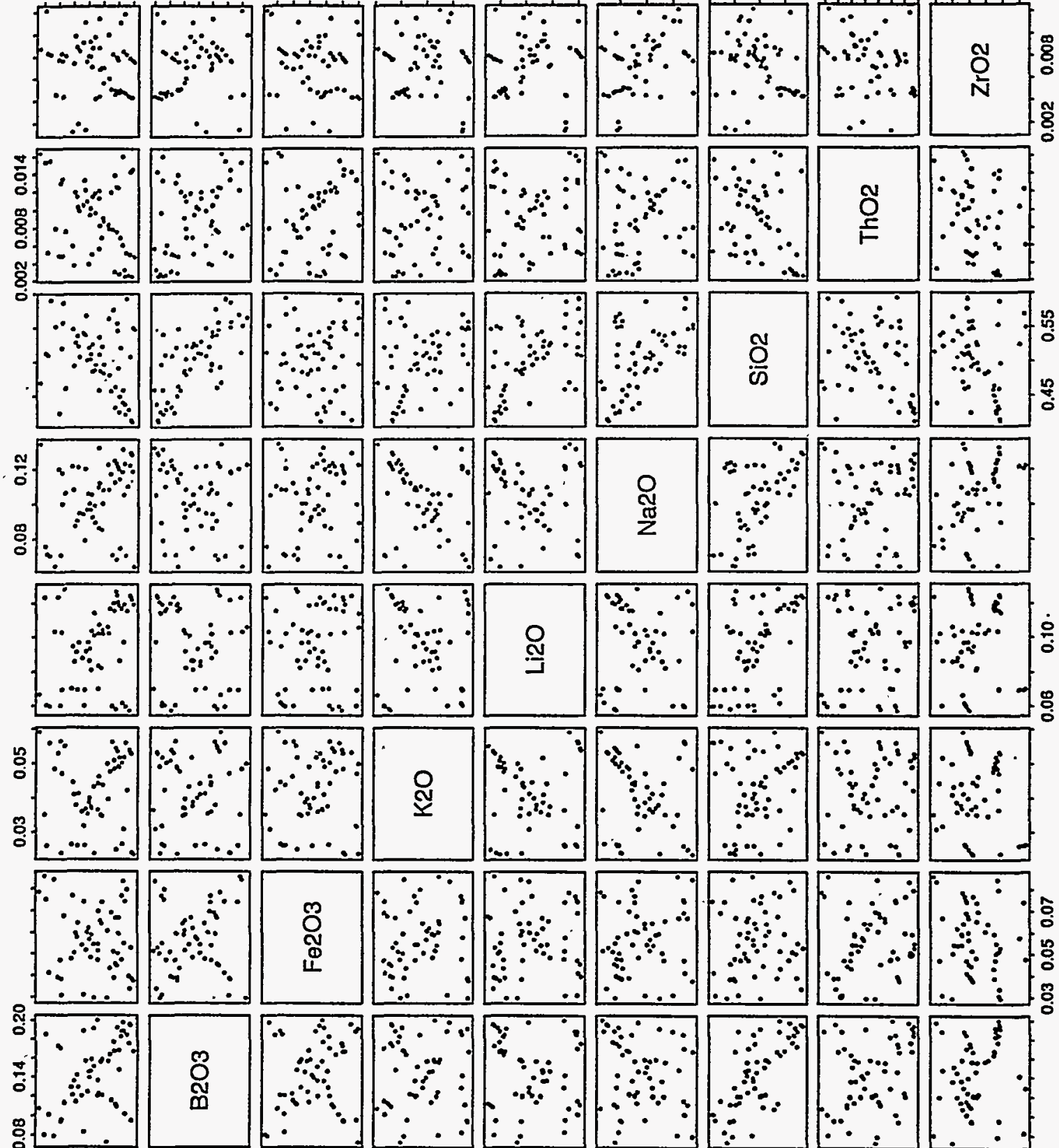

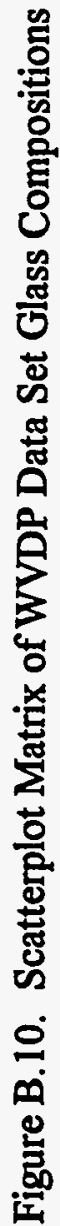
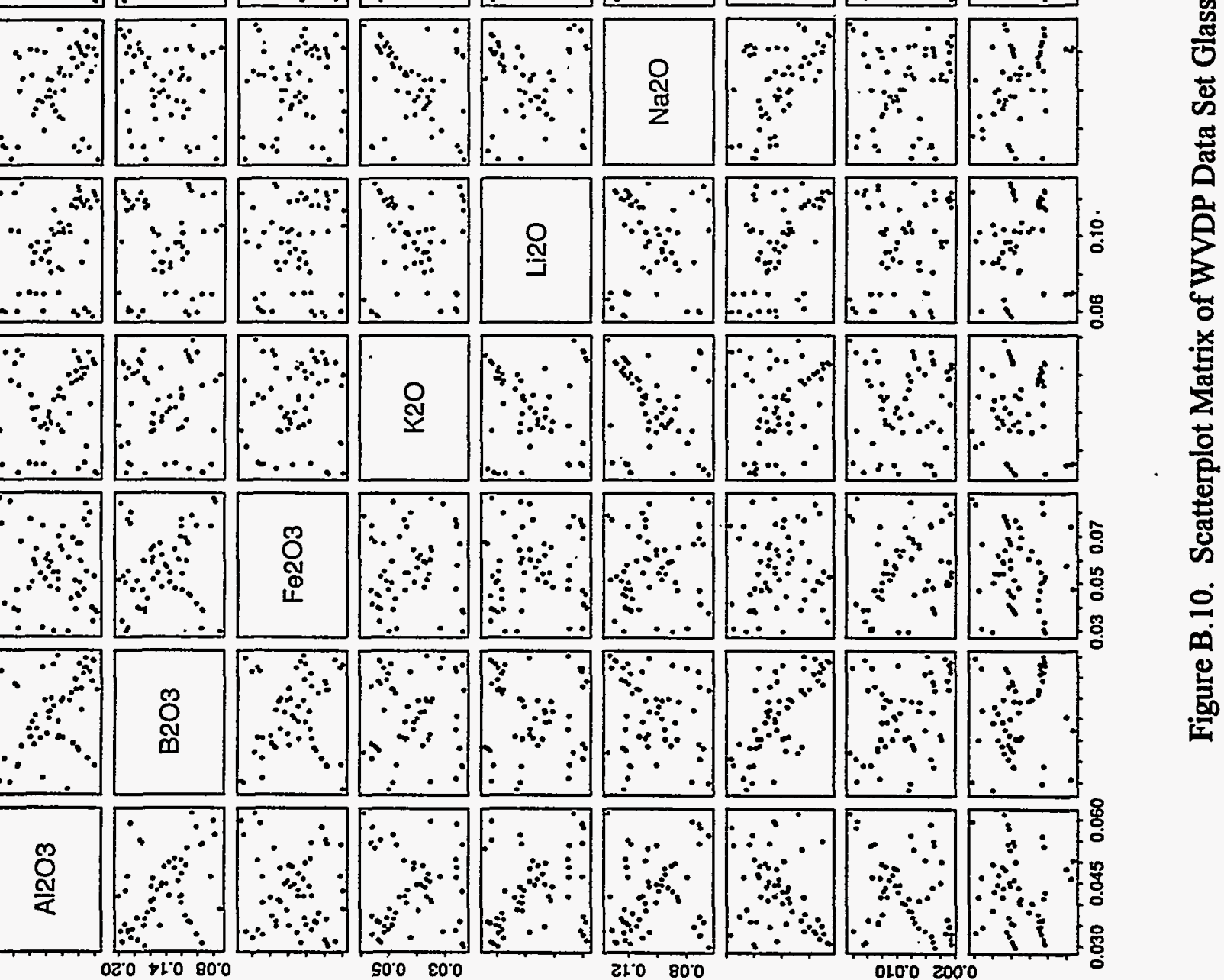


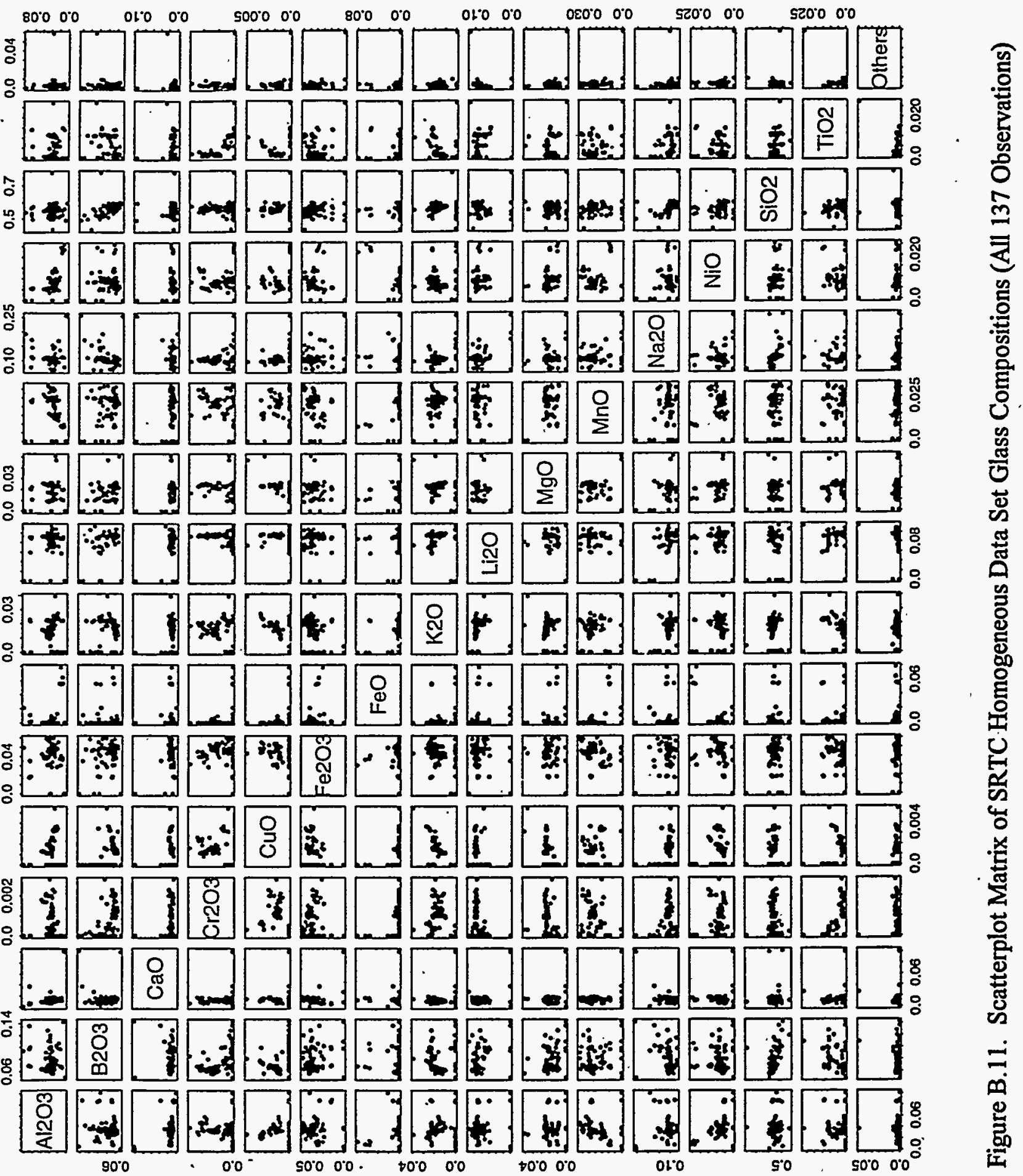




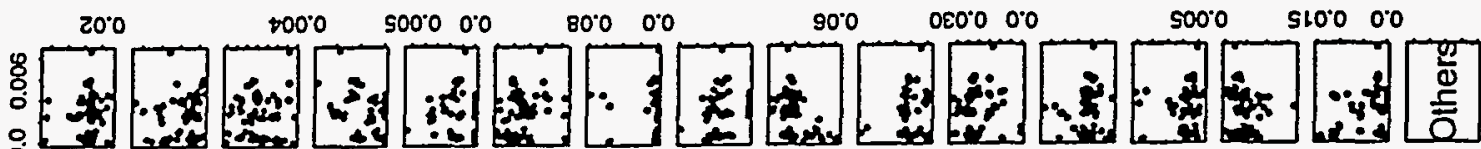

5.

- a c C

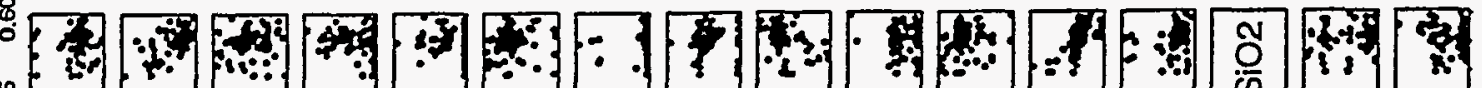

$\stackrel{?}{\circ}$

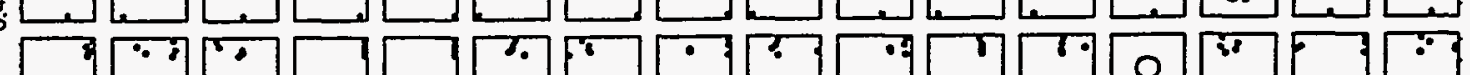

[

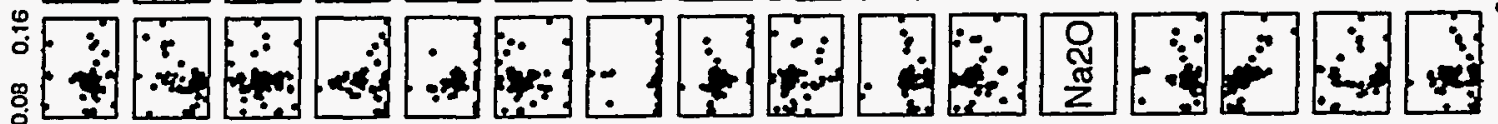

[4]

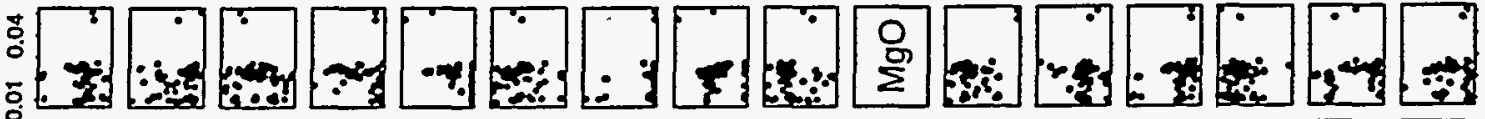

$\circ$

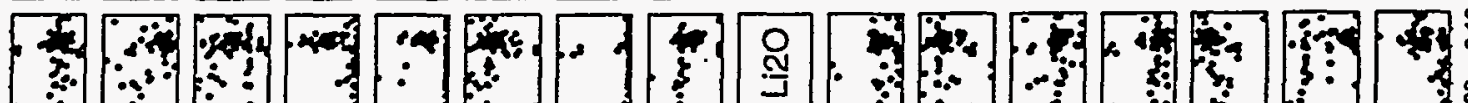

(1)

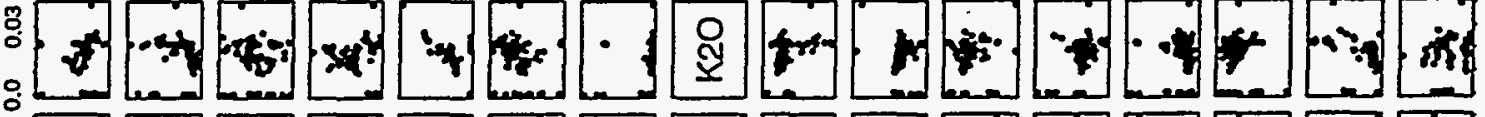

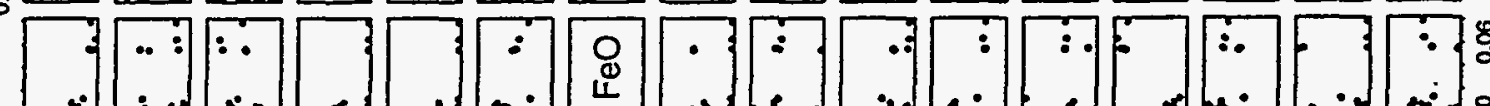

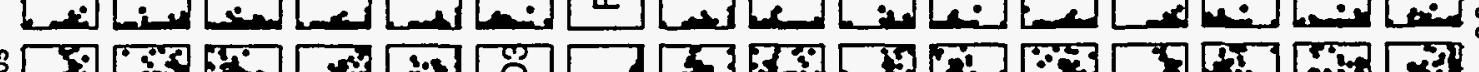

总

. . .

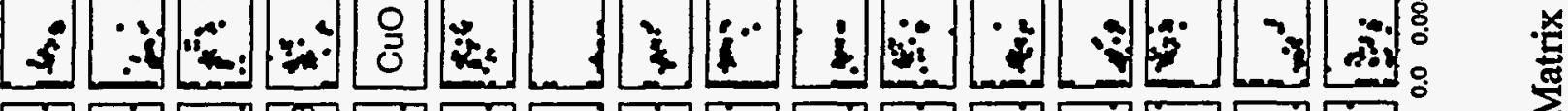

䓪

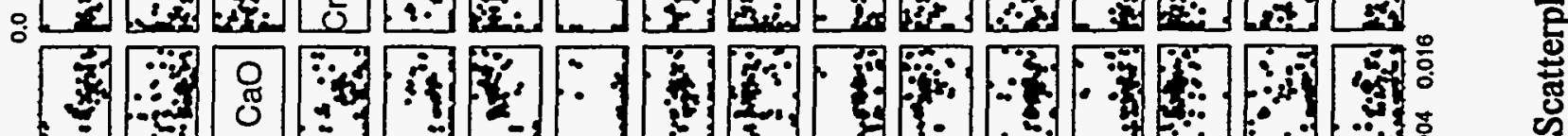

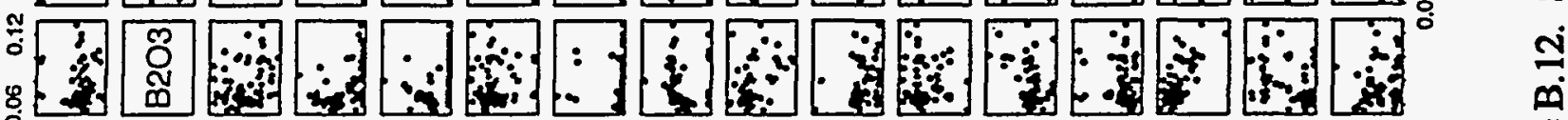

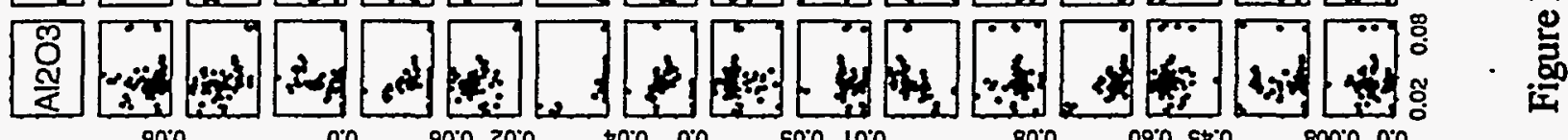




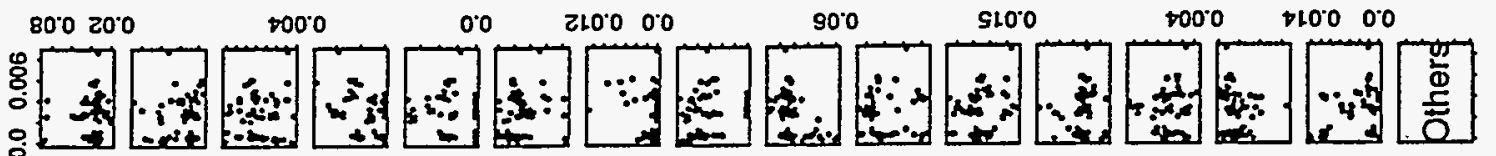

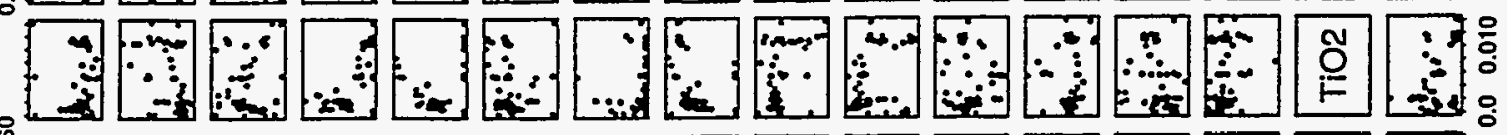

造

s.

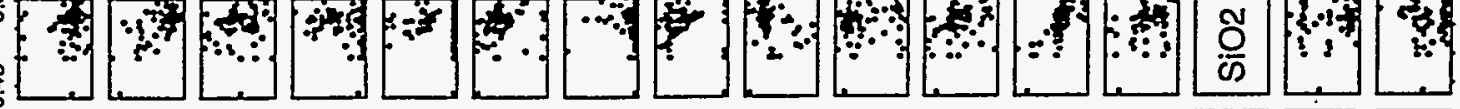

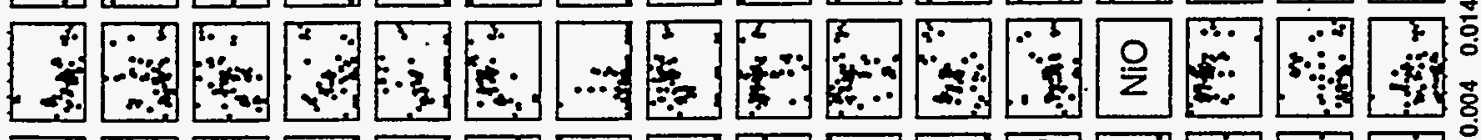

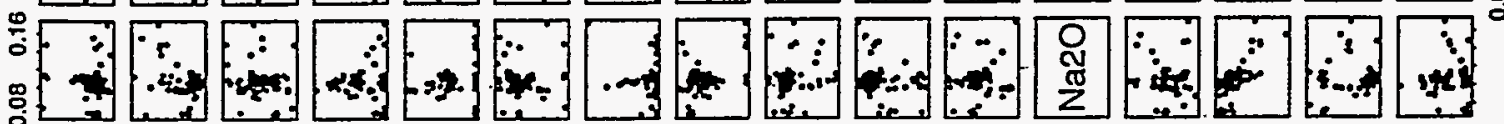

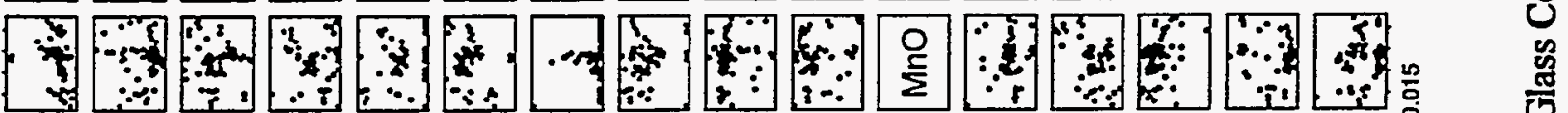

of

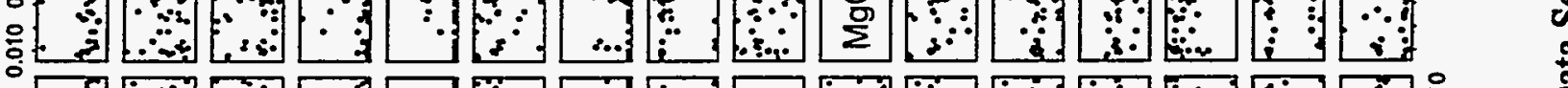

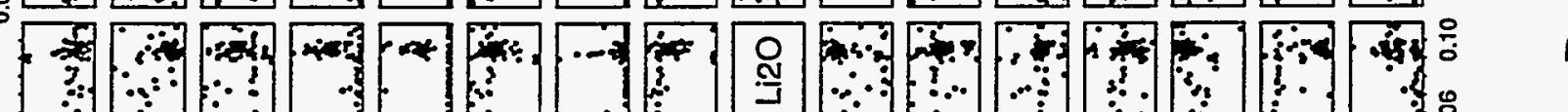

急

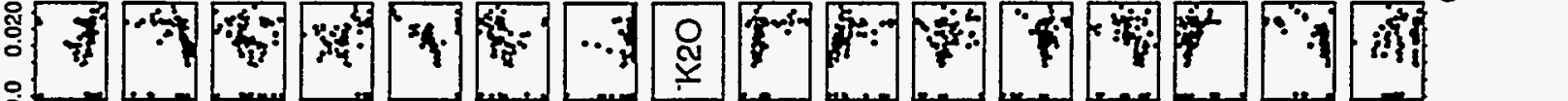

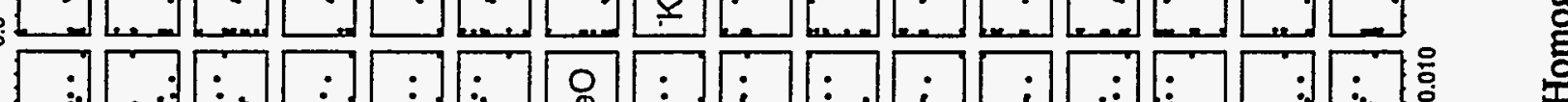

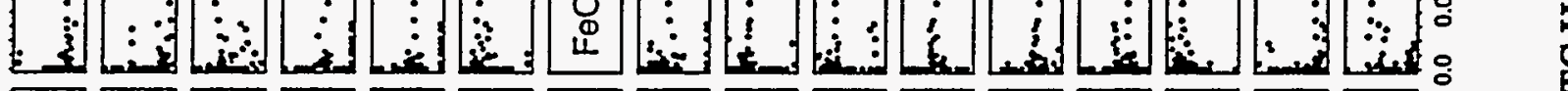

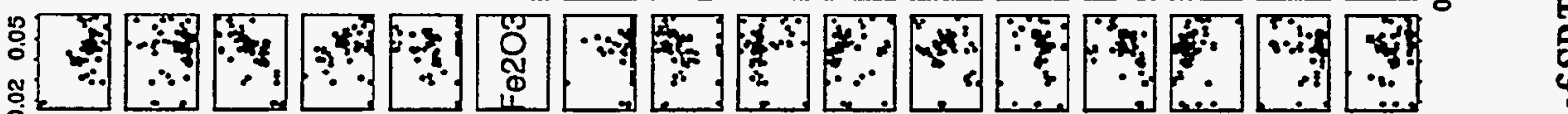

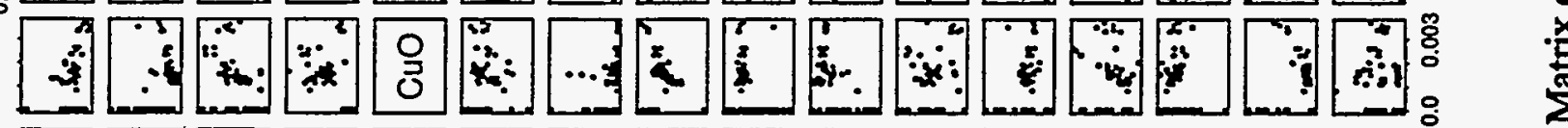

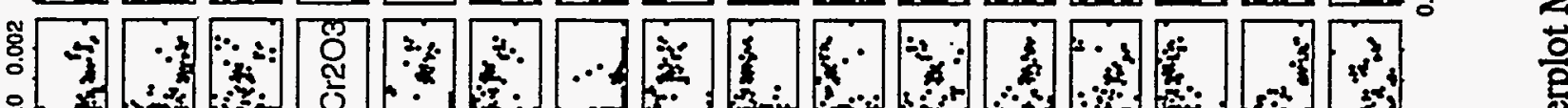

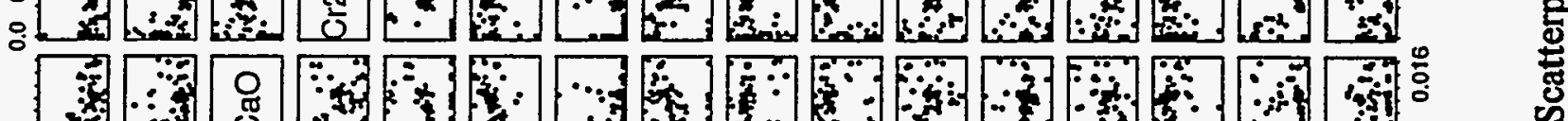

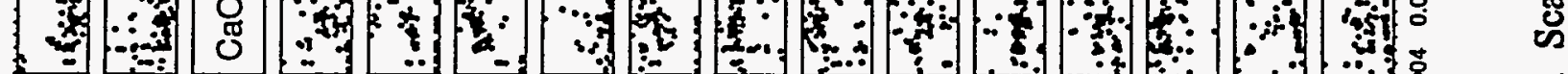

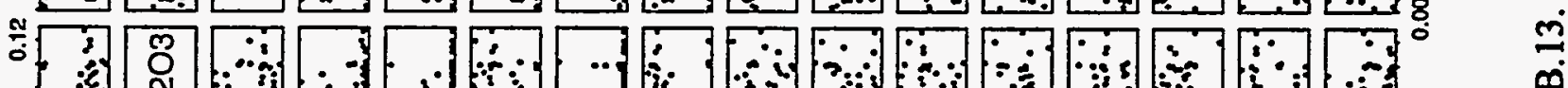

$\infty$ b 造

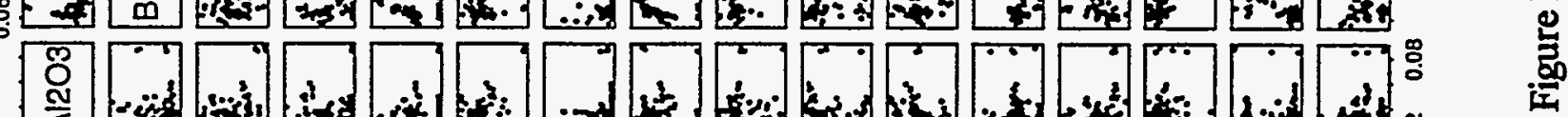

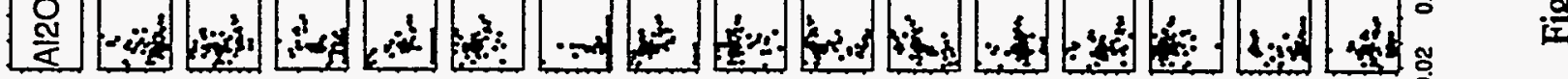

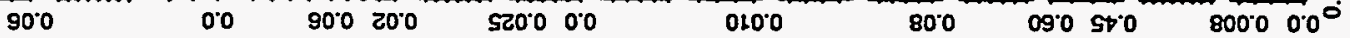




\section{Appendix' C}

\section{Predicted Versus Measured Plots}

This appendix contains the predicted versus measured $\ln (\mathrm{NR})$ plots for the free energy of hydration and first-order mixture models presented in Section 6. A predicted versus measured plot for a given model consists of a scatterplot of the $\ln (\mathrm{NR})$ values predicted by the model for each data point (on the $y$-axis) versus the measured $\ln (\mathrm{NR})$ values for each data point (on the $\mathrm{x}$ axis). Included in each predicted versus measured plot is a $45^{\circ}$ line that represents perfect prediction by the model. That is, if the model predicted the data perfectly, the predicted value would equal the measured value for each data point, and the plotted points would all fall on the $45^{\circ}$ line.

Any tendency for points to fall more above the $45^{\circ}$ line than below for a particular range of $\ln (\mathrm{NR})$ values indicates the model tends to overpredict for that range of values. Similarly, any tendency for points to fall more below the $45^{\circ}$ line than above for a particular range of $\ln (\mathrm{NR})$ values indicates the model tends to underpredict for that range of values. Ideally, a model should yield unbiased predictions (i.e., not tend to overpredict or underpredict) over its whole range of applicability.

The imprecision of a model is indicated by the scatter of predicted versus measured points about the $45^{\circ}$ line. A very tight scatter about the $45^{\circ}$ line indicates the model's predictions are very precise (i.e., have low uncertainty). A very wide scatter about the $45^{\circ}$ line indicates the model's predictions are not very precise (i.e., have high uncertainty). Ideally a model should yield very precise predictions. 
Measured PCT Boron Release $\left(\mathrm{g} / \mathrm{m}^{2}\right)$

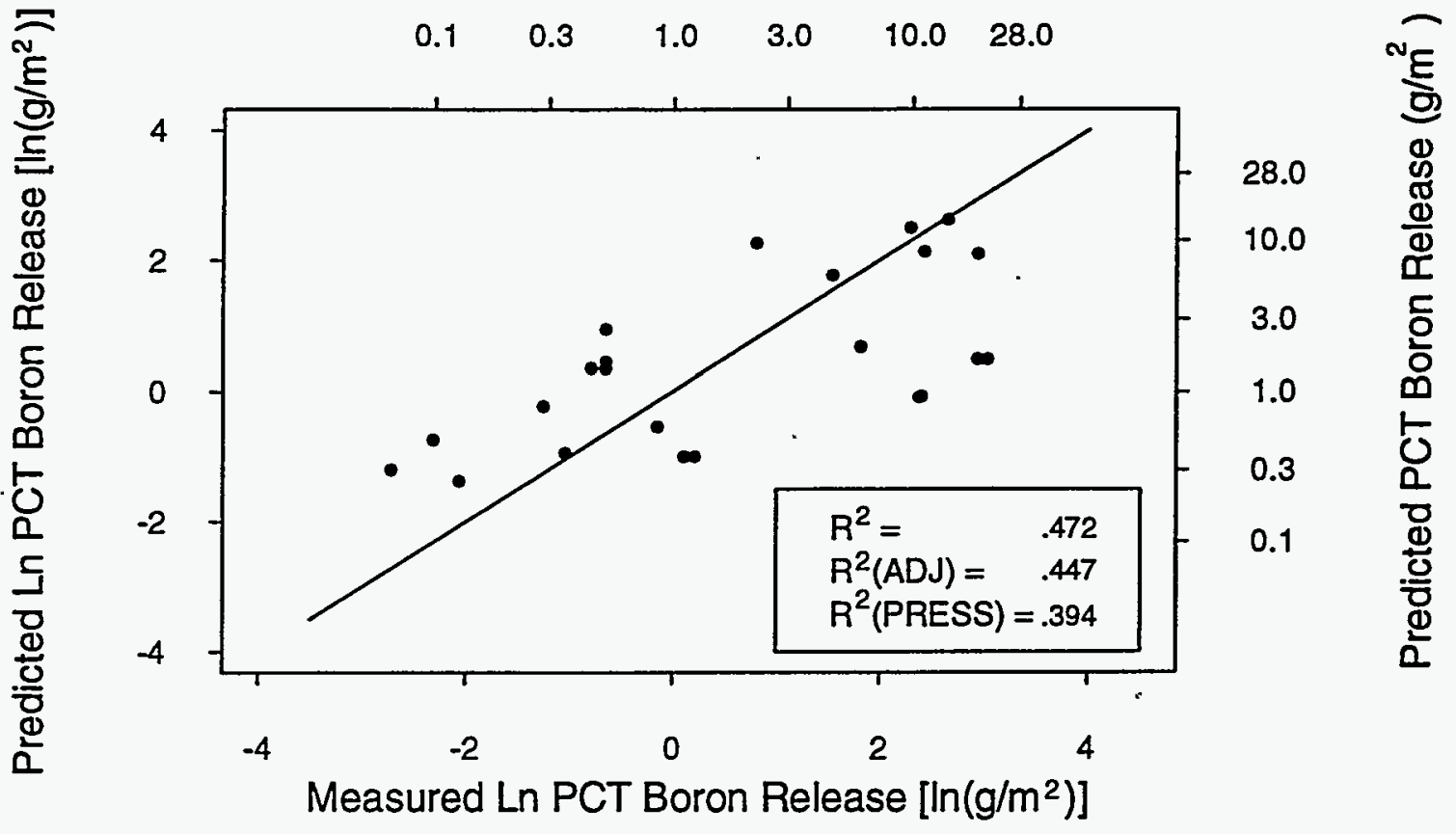

Figure C.1. Predicted Versus Measured Plot for the Free Energy of Hydration Model Fitted to the CVS-I Data

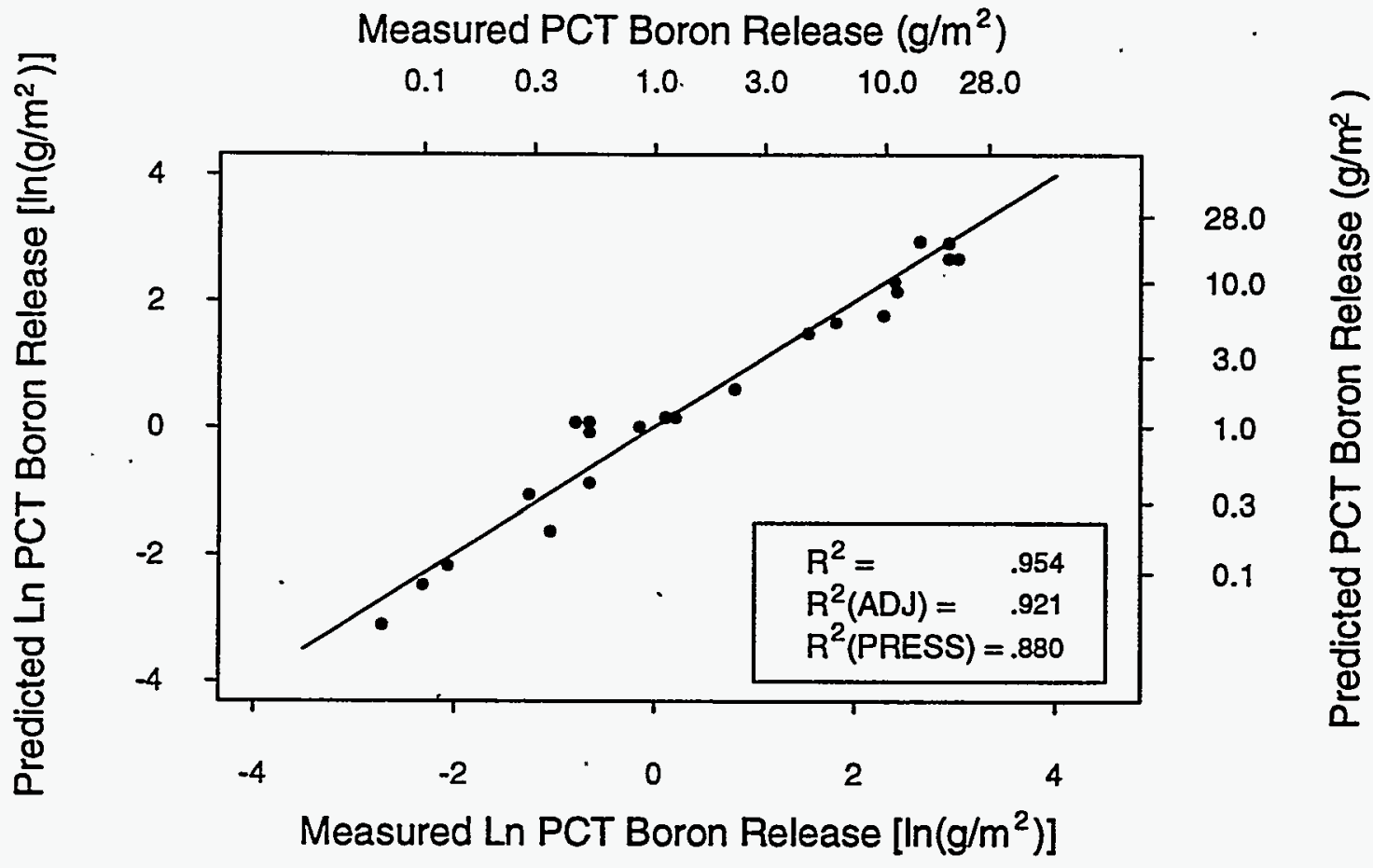

Figure C.2. Predicted Versus Measured Plot for the First-Order Mixture Model Fitted to the CVS-I Data 
Measured PCT Boron Release $\left(\mathrm{g} / \mathrm{m}^{2}\right)$

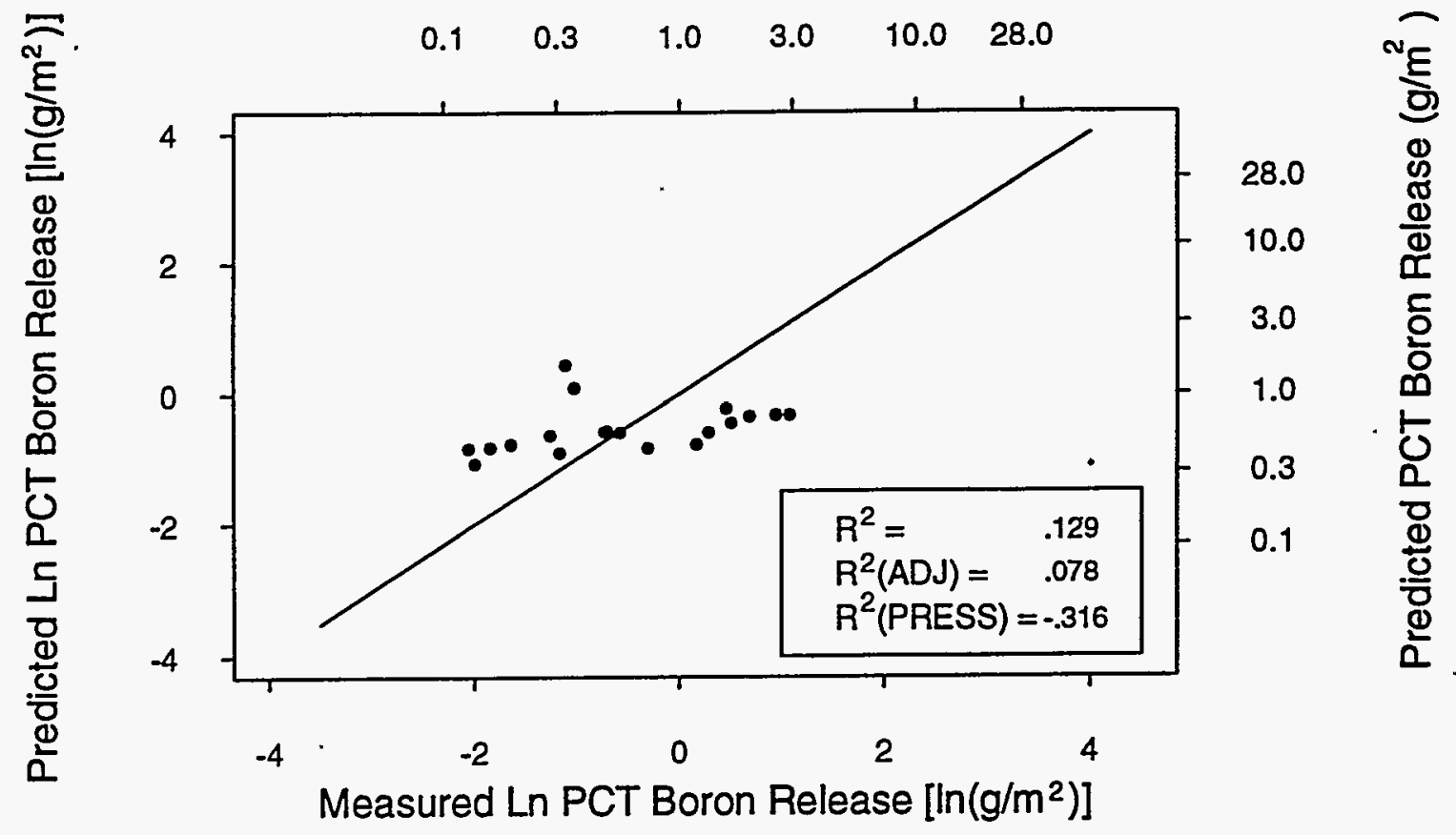

Figure C.3. Predicted Versus Measured Plot for the Free Energy of Hydration Model Fitted to the.CVS-II Phase 1 Data

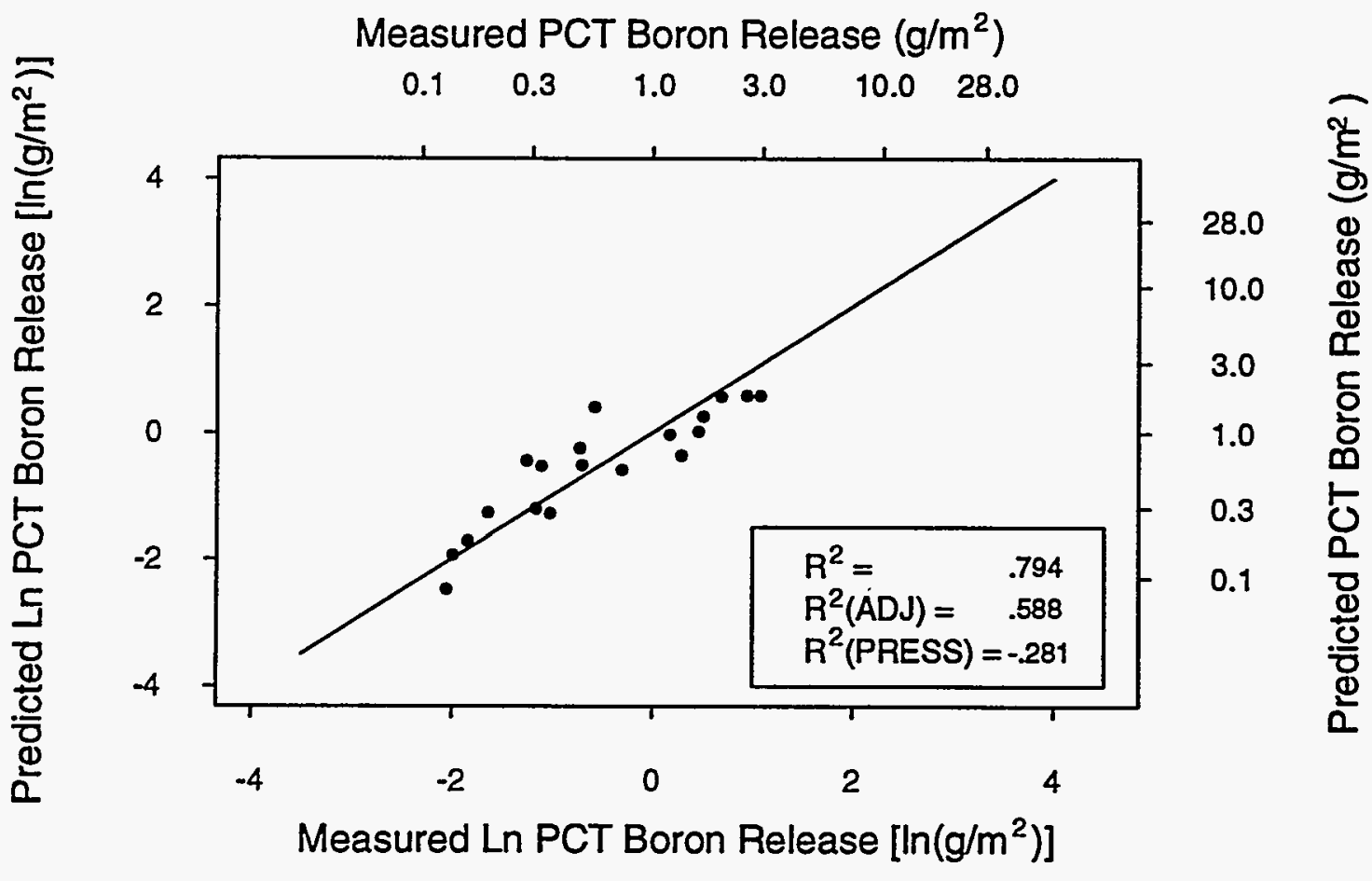

Figure C.4. Predicted Versus Measured Plot for the First-Order Mixture Model Fitted to the CVS-II Phase 1 Data 
Measured PCT Boron Release $\left(\mathrm{g} / \mathrm{m}^{2}\right)$

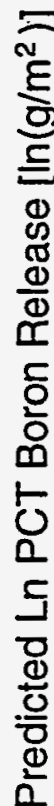

$\begin{array}{llllll}0.1 & 0.3 & 1.0 & 3.0 & 10.0 & 28.0\end{array}$

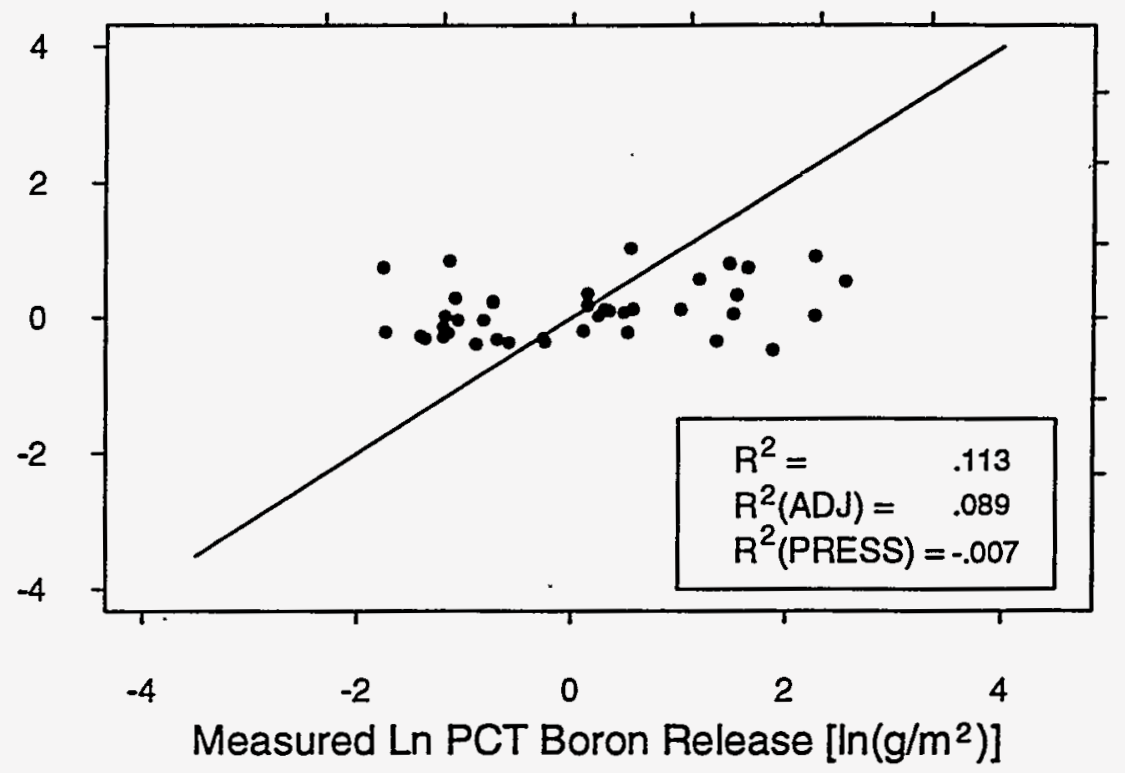

28.0

10.0

3.0

1.0

0.3

0.1

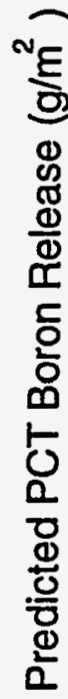

Figure C.5. Predicted Versus Measured Plot for the Free Energy of Hydration Model Fitted to the CVS-II Phase 2 Data

Measured PCT Boron Release $\left(\mathrm{g} / \mathrm{m}^{2}\right)$

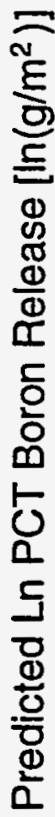

$\begin{array}{llllll}0.1 & 0.3 & 1.0 & 3.0 & 10.0 & 28.0\end{array}$

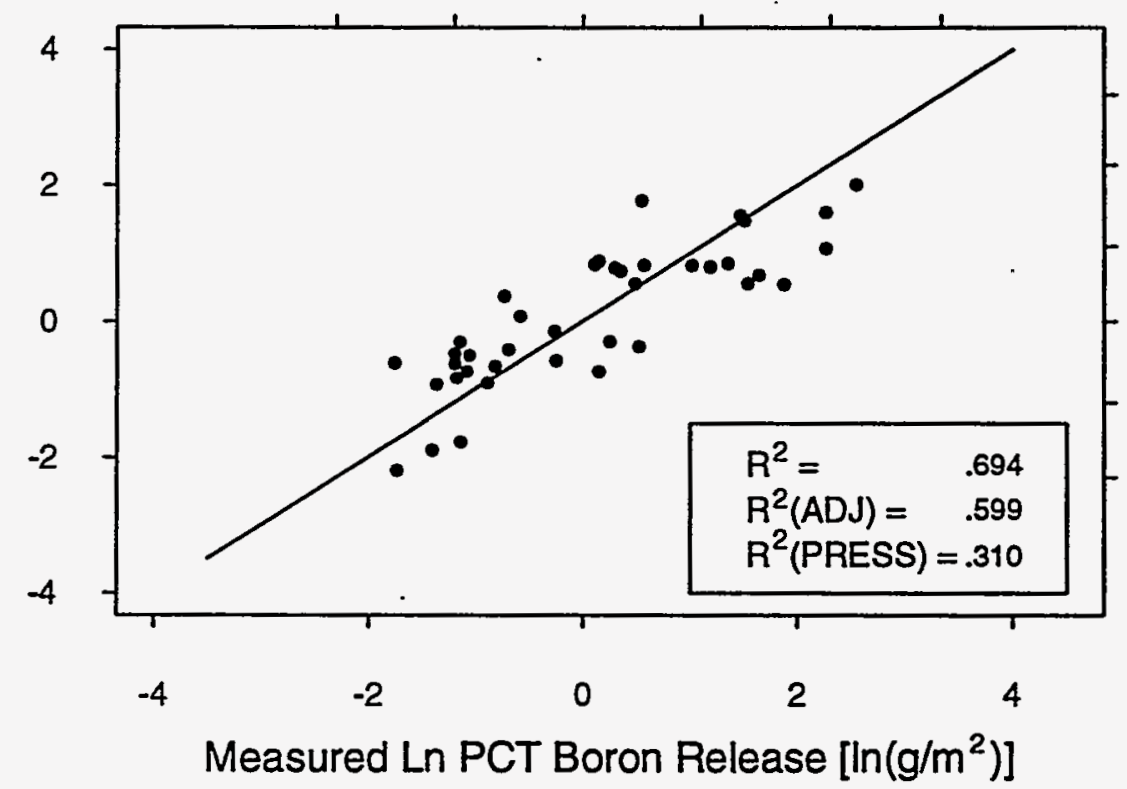

28.0

10.0

3.0

1.0

0.3

0.1

है

ฆ

它

음

Figure C.6. Predicted Versus Measured Plot for the First-Order Mixture Model Fitted to the CVS-II Phase 2 Data 
Measured PCT Boron Release $\left(\mathrm{g} / \mathrm{m}^{2}\right)$

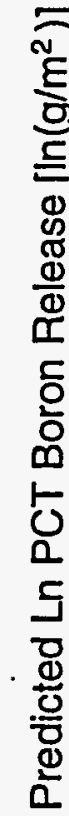

$\begin{array}{llllll}0.1 & 0.3 & 1.0 & 3.0 & 10.0 & 28.0\end{array}$

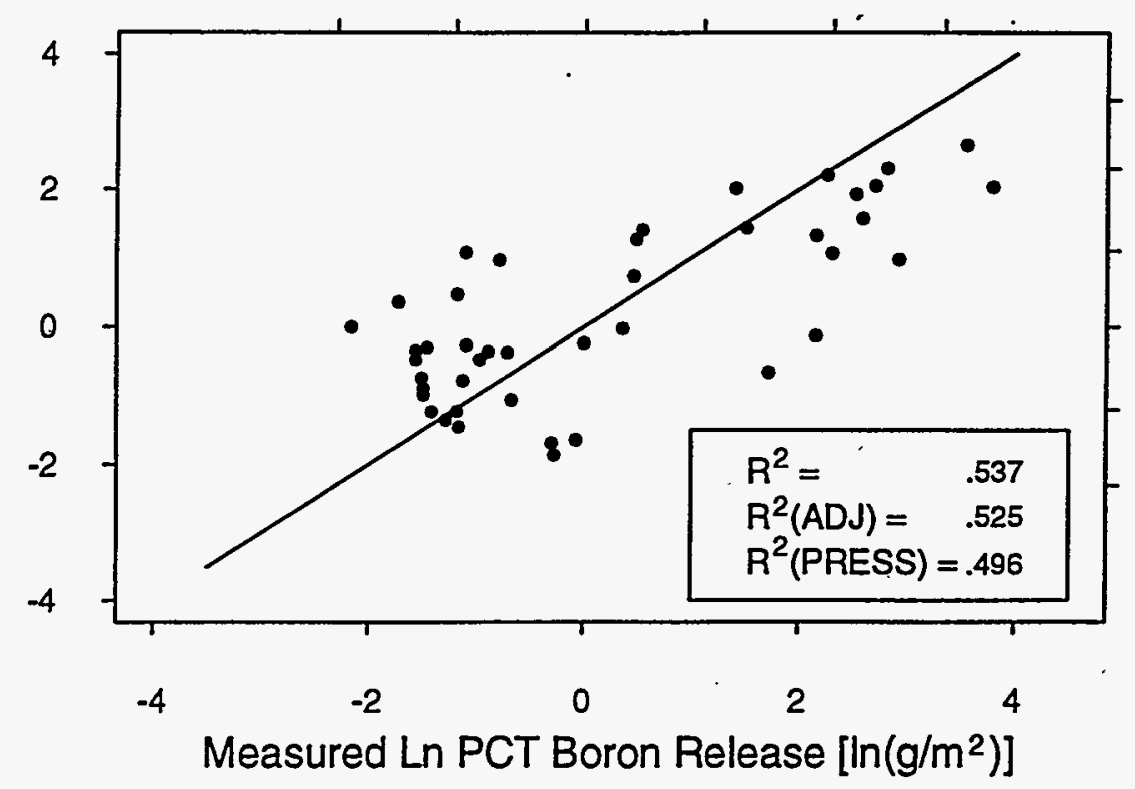

28.0

10.0

3.0

1.0

0.3

0.1

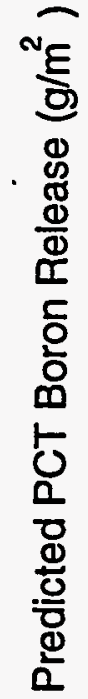

Figure C.7. Predicted Versus Measured Plot for the Free Energy of Hydration Model Fitted to the CVS-II Phase 3 Data

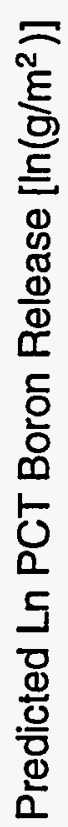

Measured PCT Boron Release $\left(\mathrm{g} / \mathrm{m}^{2}\right)$.

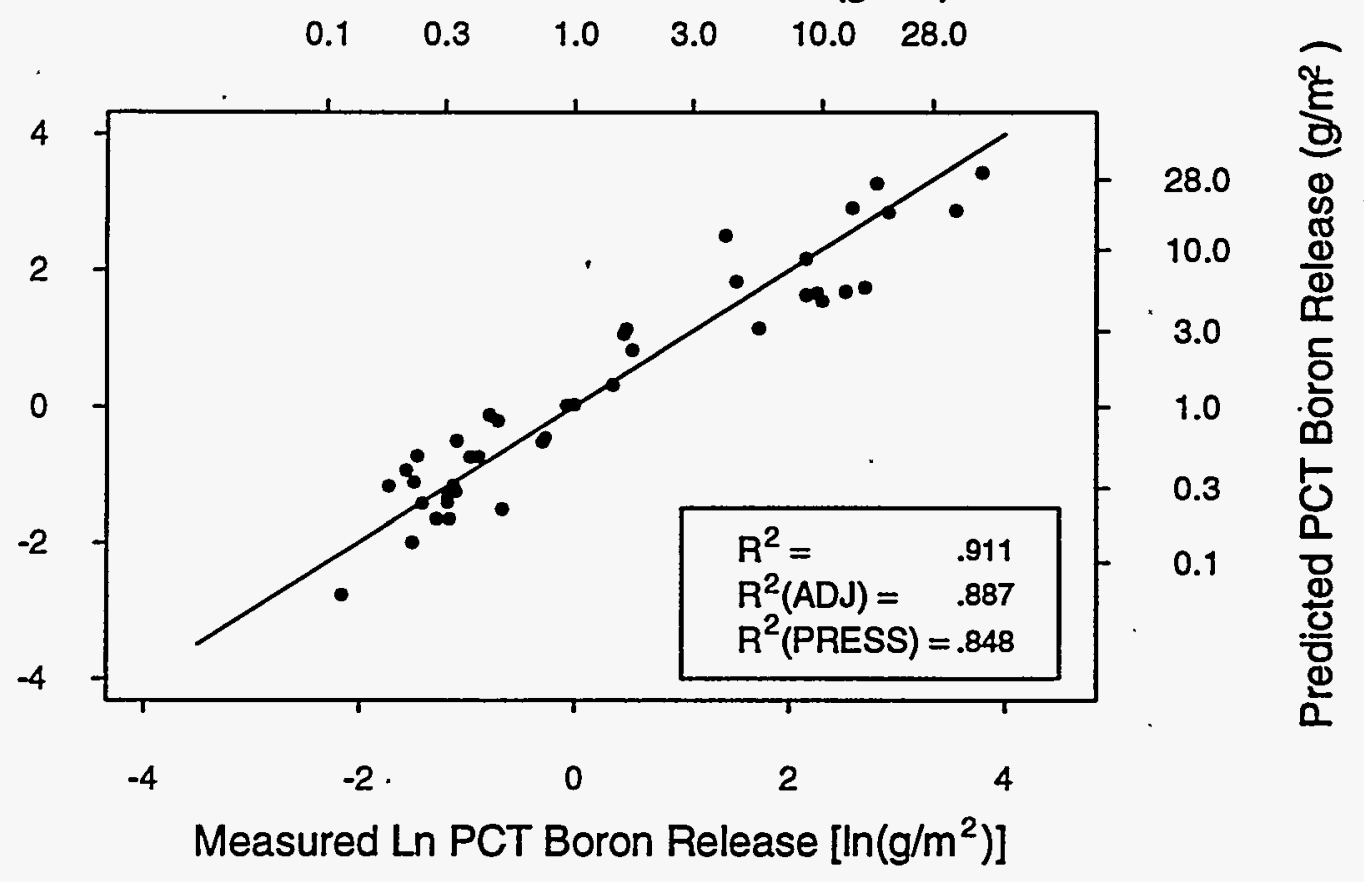

Figure C.8. Predicted Versus Measured Plot for the First-Order Mixture Model Fitted to the CVS-II Phase 3 Data 
Measured PCT Boron Release $\left(\mathrm{g} / \mathrm{m}^{2}\right)$
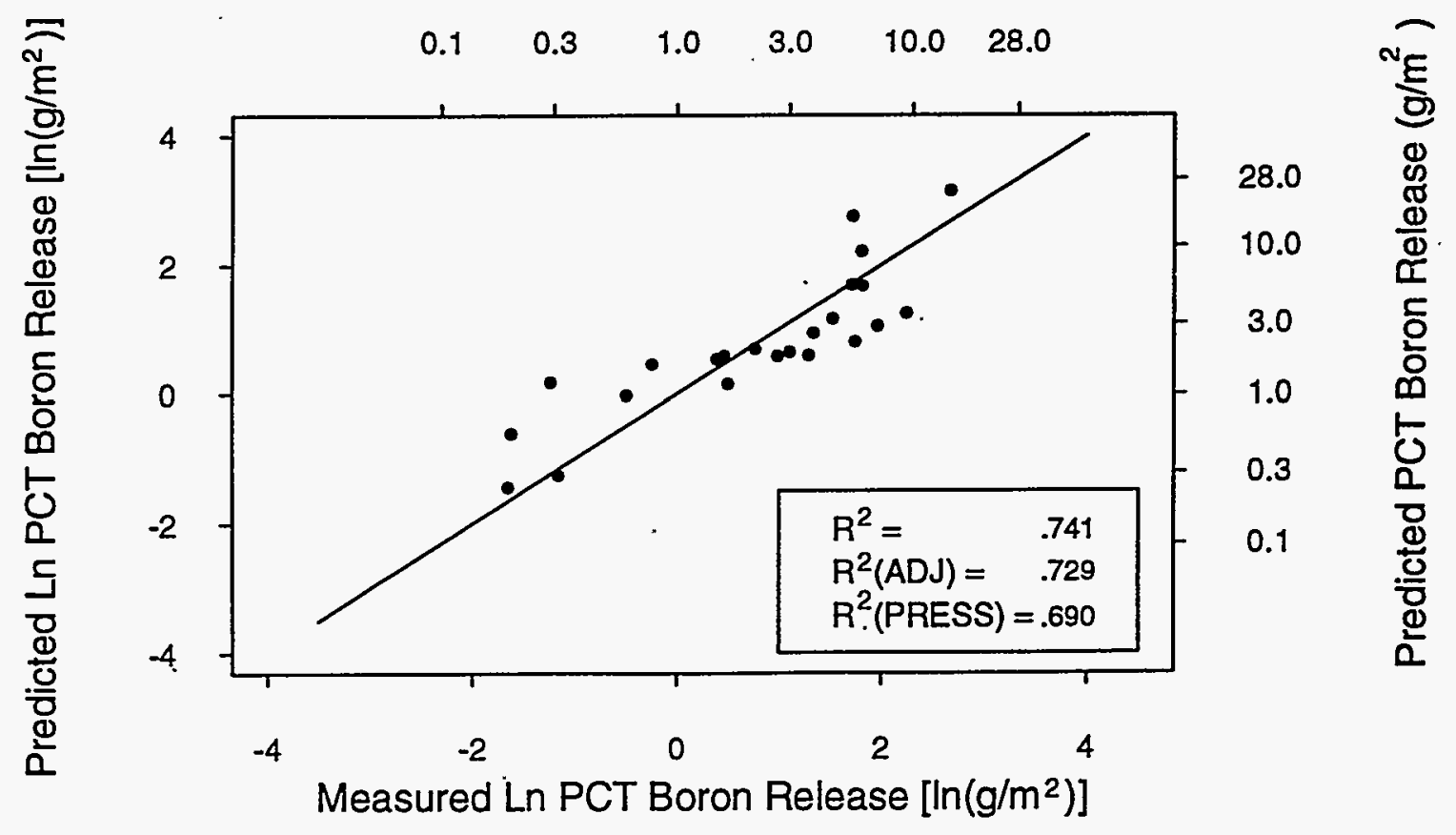

Figure C.9. Predicted Versus Measured Plot for the Free Energy of Hydration Model Fitted to the CVS-II Phase 4 Data

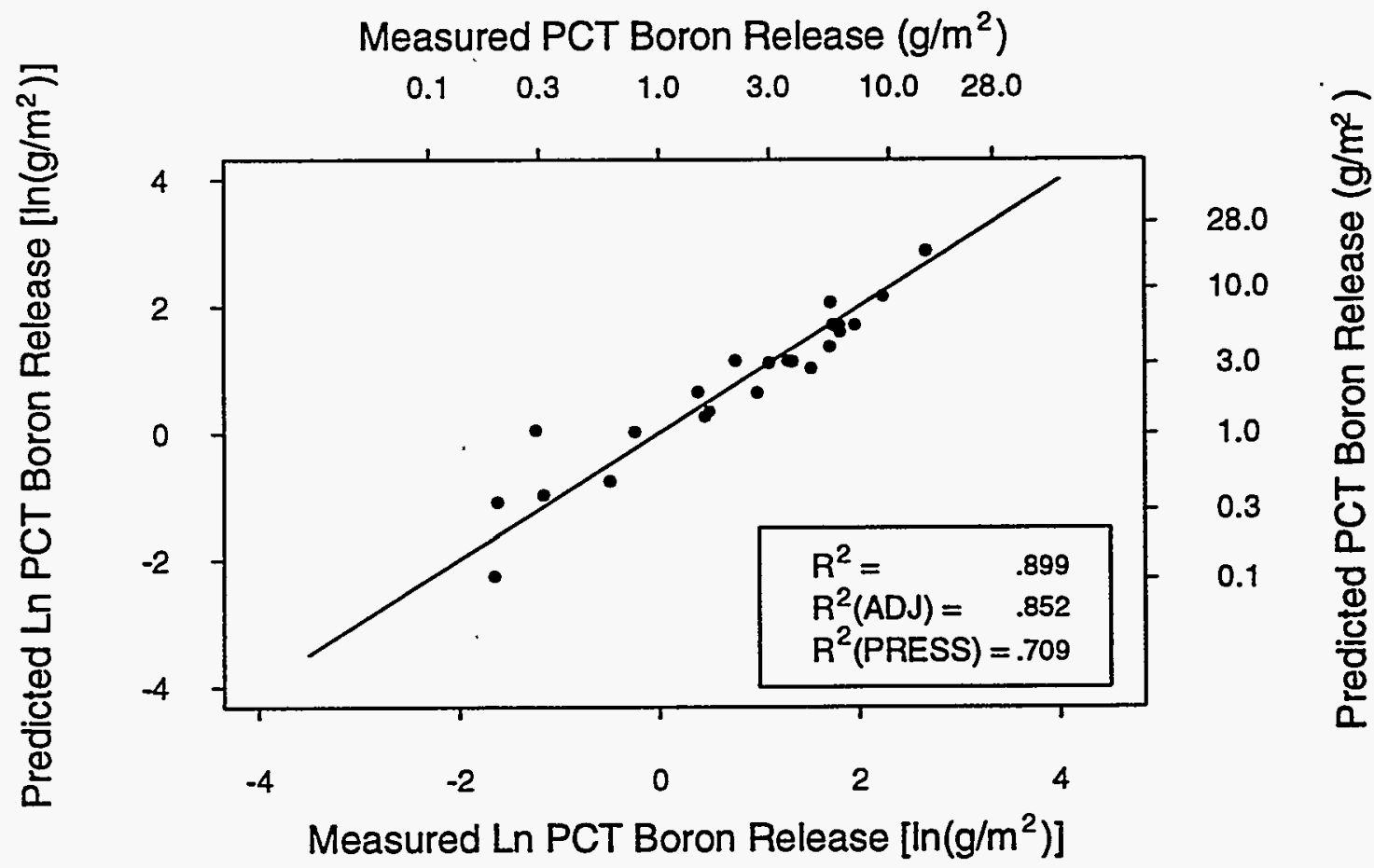

Figure C.10. Predicted Versus Measured Plot for the First-Order Mixture Model Fitted to the CVS-II Phase 4 Data 
Measured PCT Boron Release $\left(\mathrm{g} / \mathrm{m}^{2}\right)$

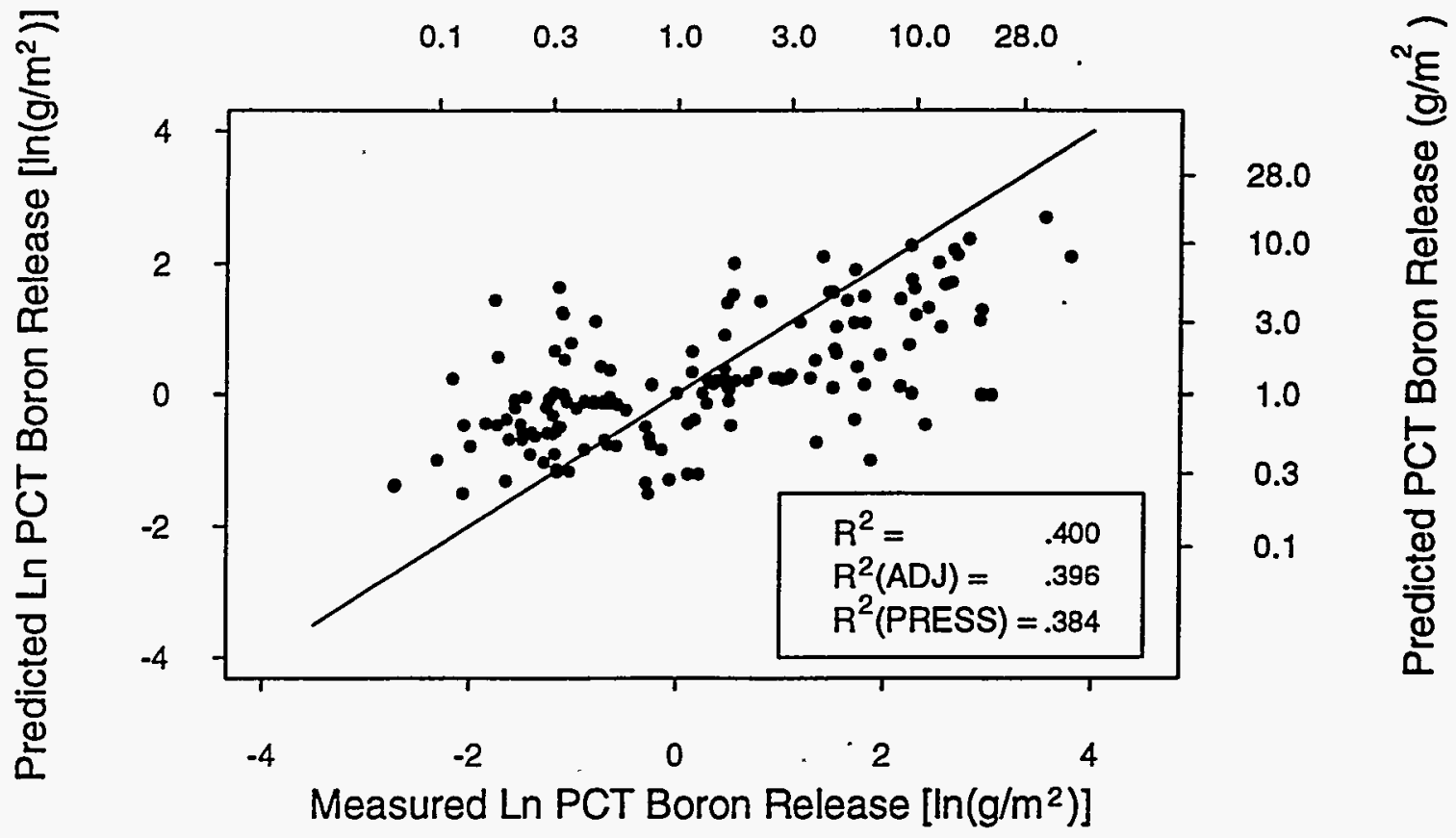

Figure C.11. Predicted Versus Measured Plot for the Free Energy of Hydration Model Fitted to All of the CVS Data

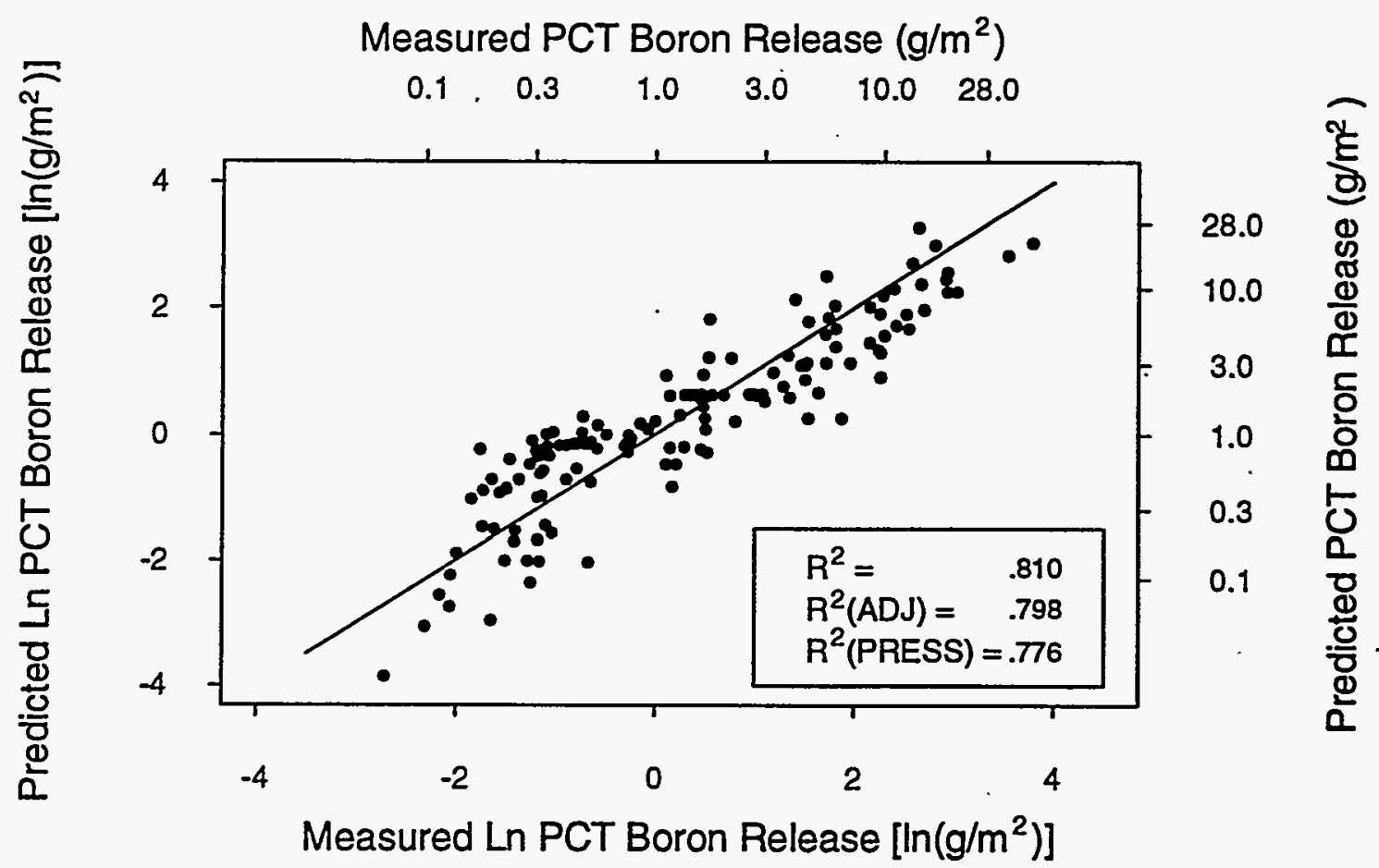

Figure C.12. Predicted Versus Measured Plot for the First-Order Mixture Model Fitted to All of the CVS Data 
Measured PCT Boron Release $\left(\mathrm{g} / \mathrm{m}^{2}\right)$
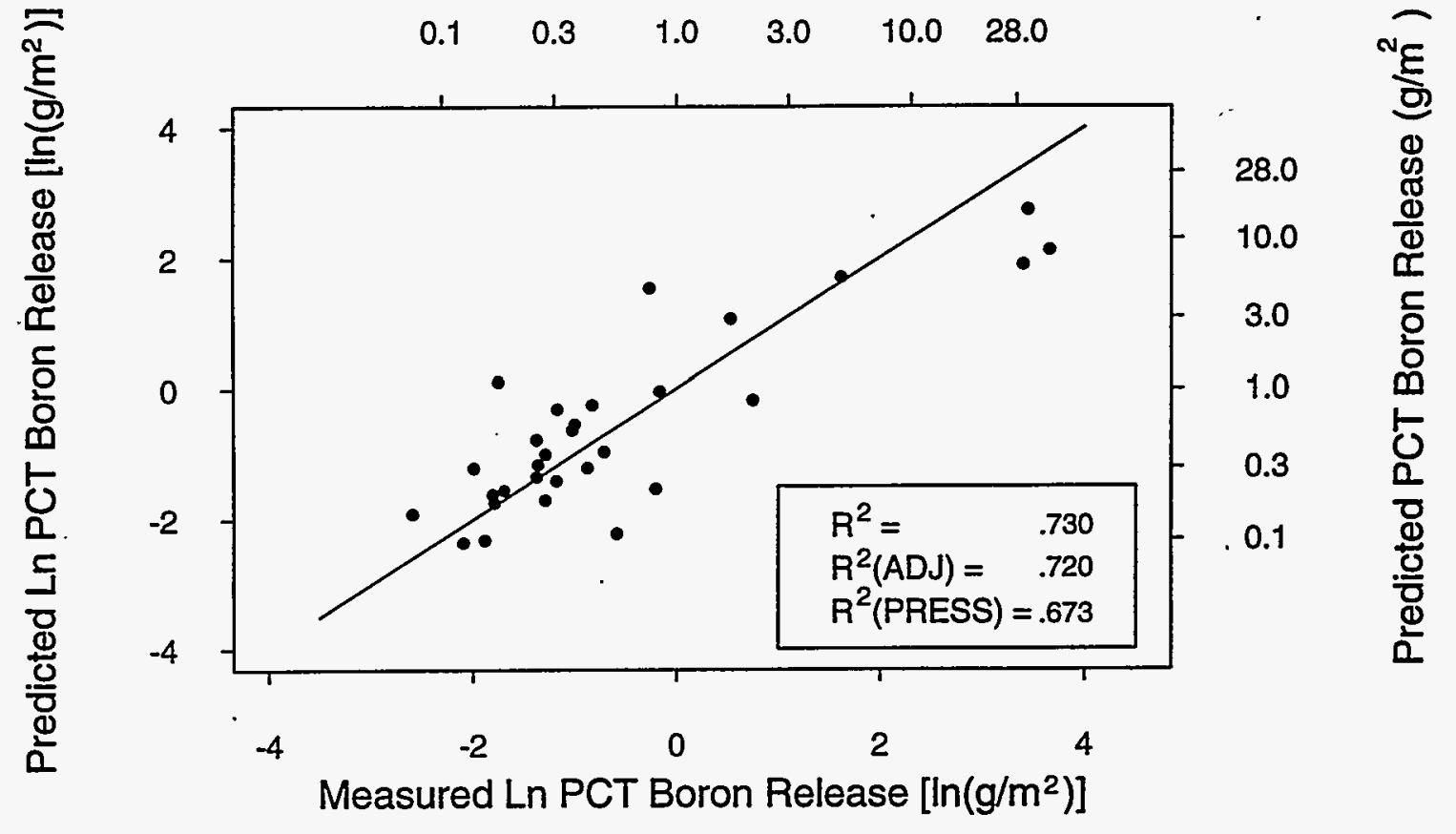

Figure C.13. Predicted Versus Measured Plot for the Free Energy of Hydration Model Fitted to the Ramsey Data

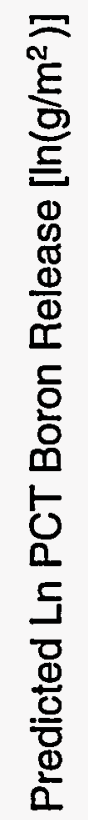

Measured PCT Boron Release $\left(\mathrm{g} / \mathrm{m}^{2}\right)$

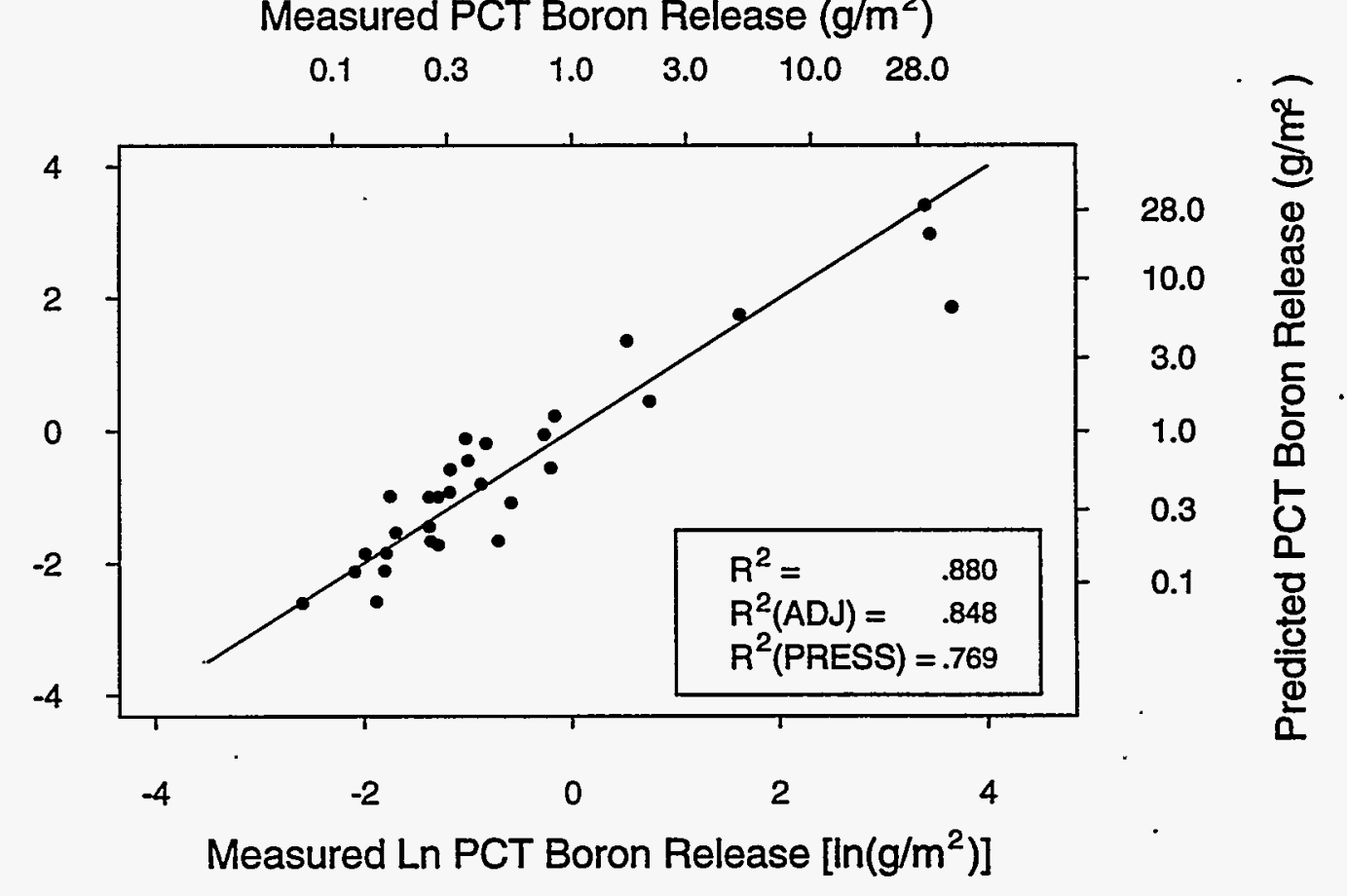

Figure C.14. Predicted Versus Measured Plot for the First-Order Mixture Model Fitted to the Ramsey Data 


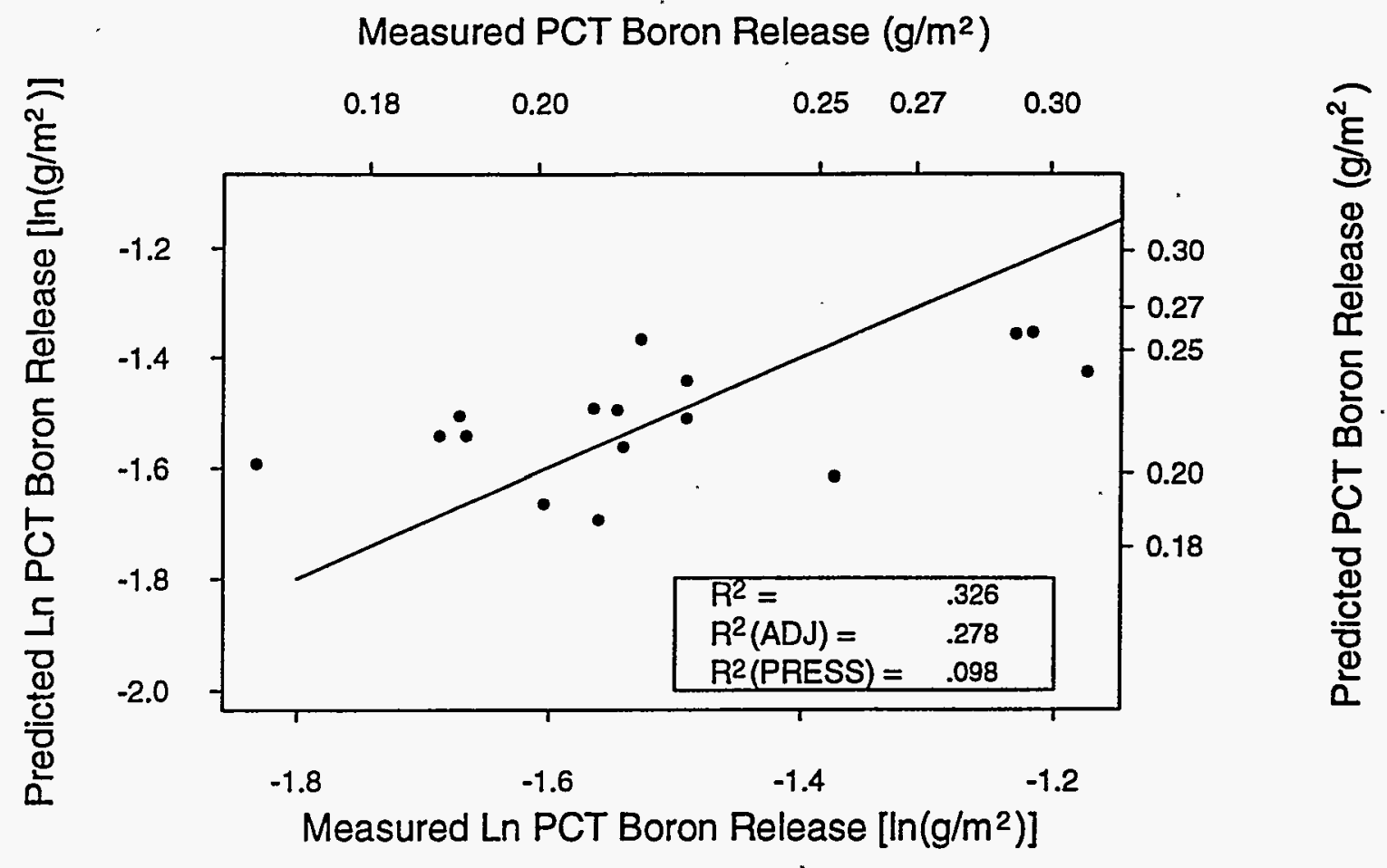

Figure C.15. Predicted Versus Measured Plot for the Free Energy of Hydration Model Fitted to the WV87 Data

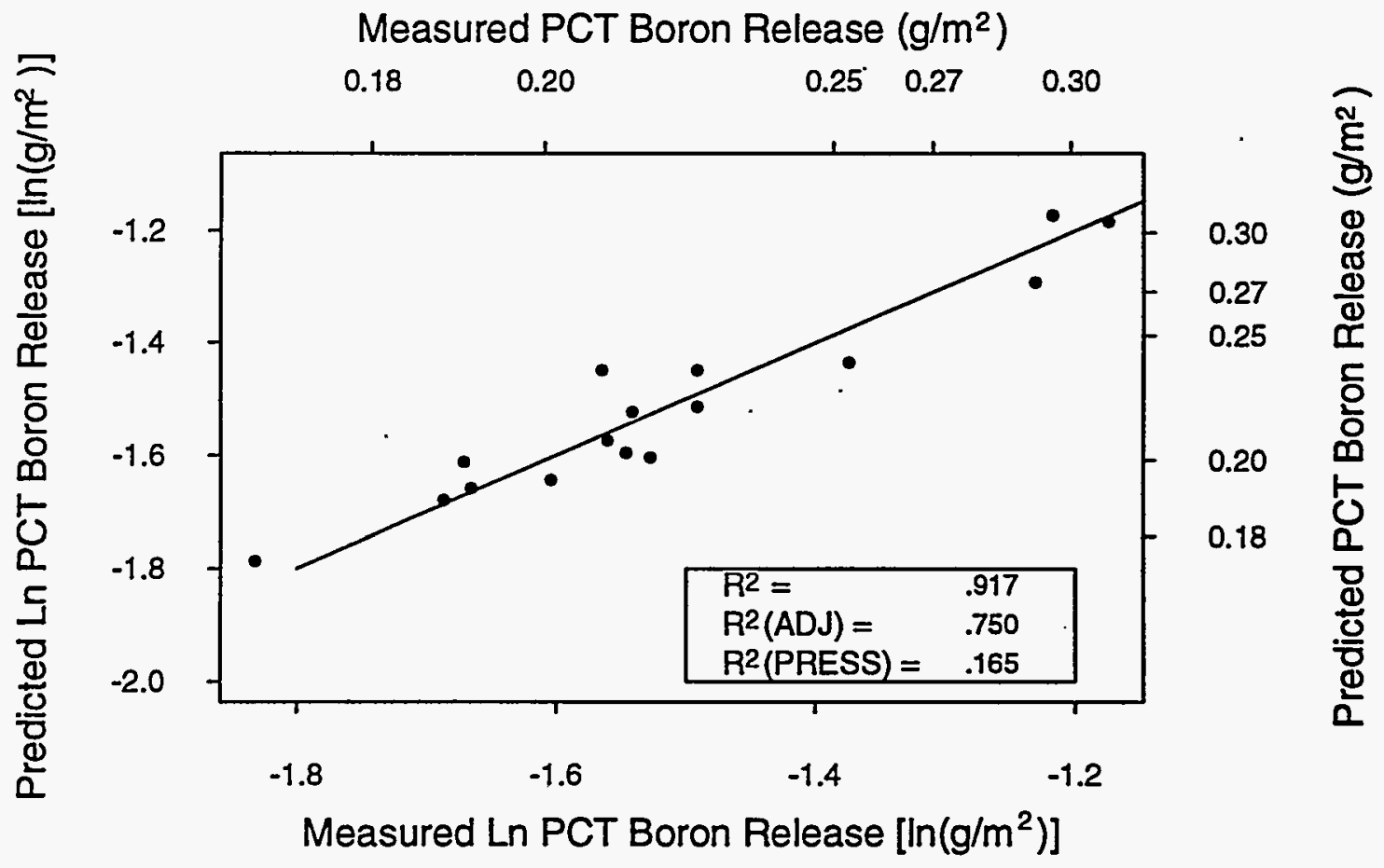

Figure C.16. Predicted Versus Measured Plot for the First-Order Mixture Model Fitted to the WV87 Data 
Measured PCT Boron Release $\left(\mathrm{g} / \mathrm{m}^{2}\right)$

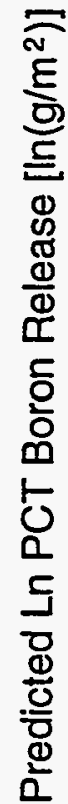

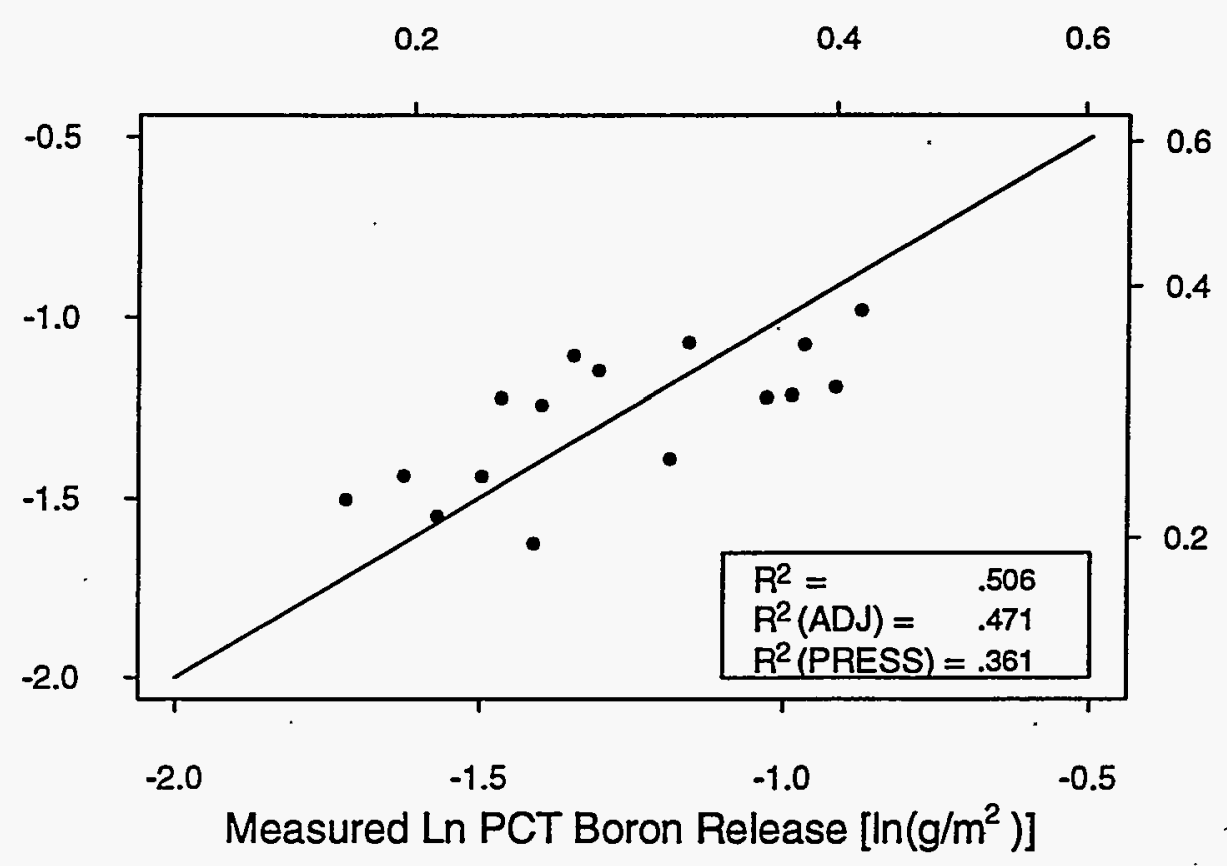

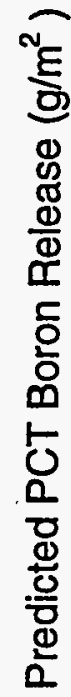

Figure C.17. Predicted Versus Measured Plot for the Free Energy of Hydration Model Fitted to the WV88 Data

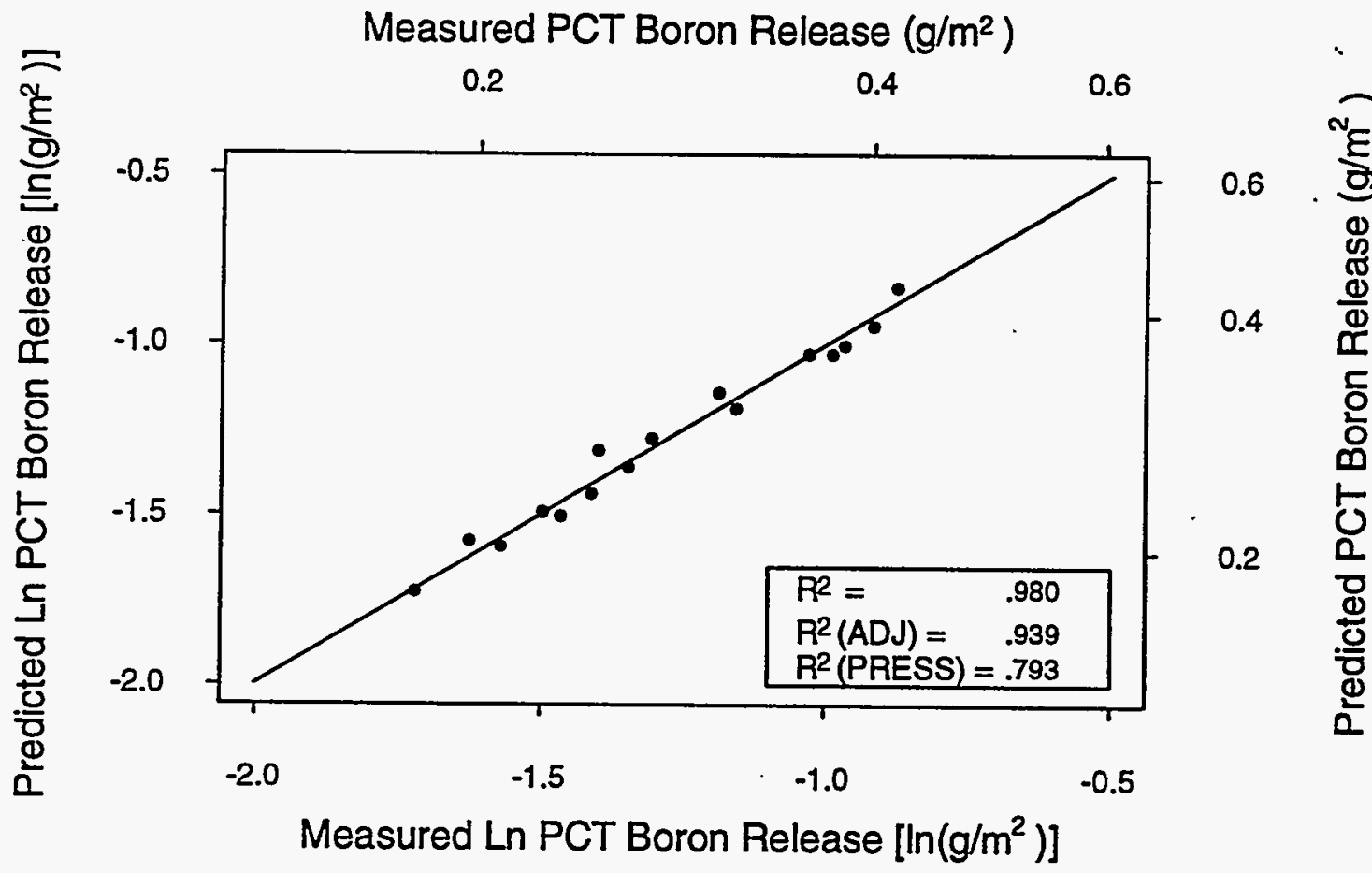

Figure C.18. Predicted Versus Measured Plot for the First-Order Mixture Model Fitted to the WV88 Data 


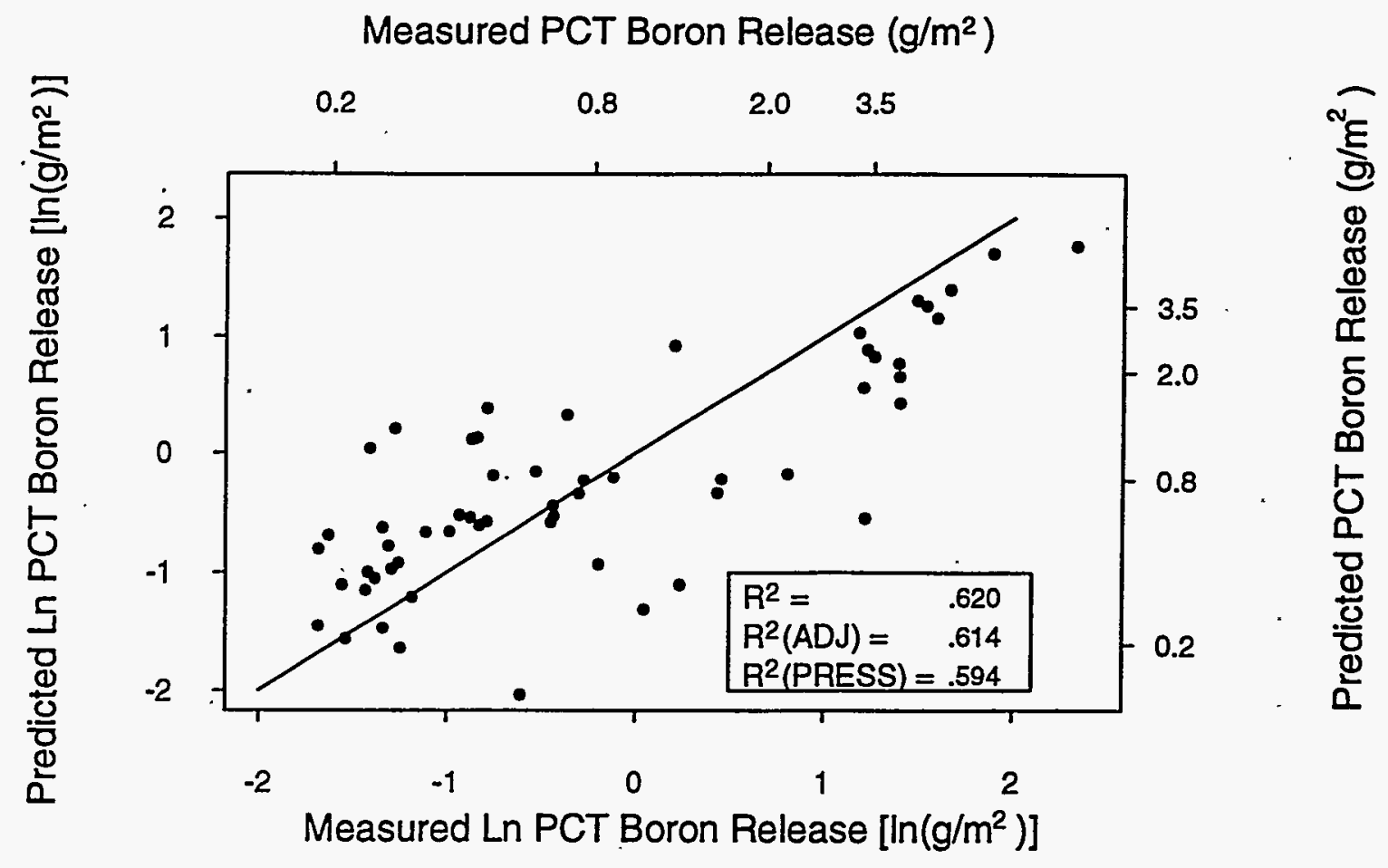

Figure C.19. Predicted Versus Measured Plot for the Free Energy of Hydration Model Fitted to the WVDP Data

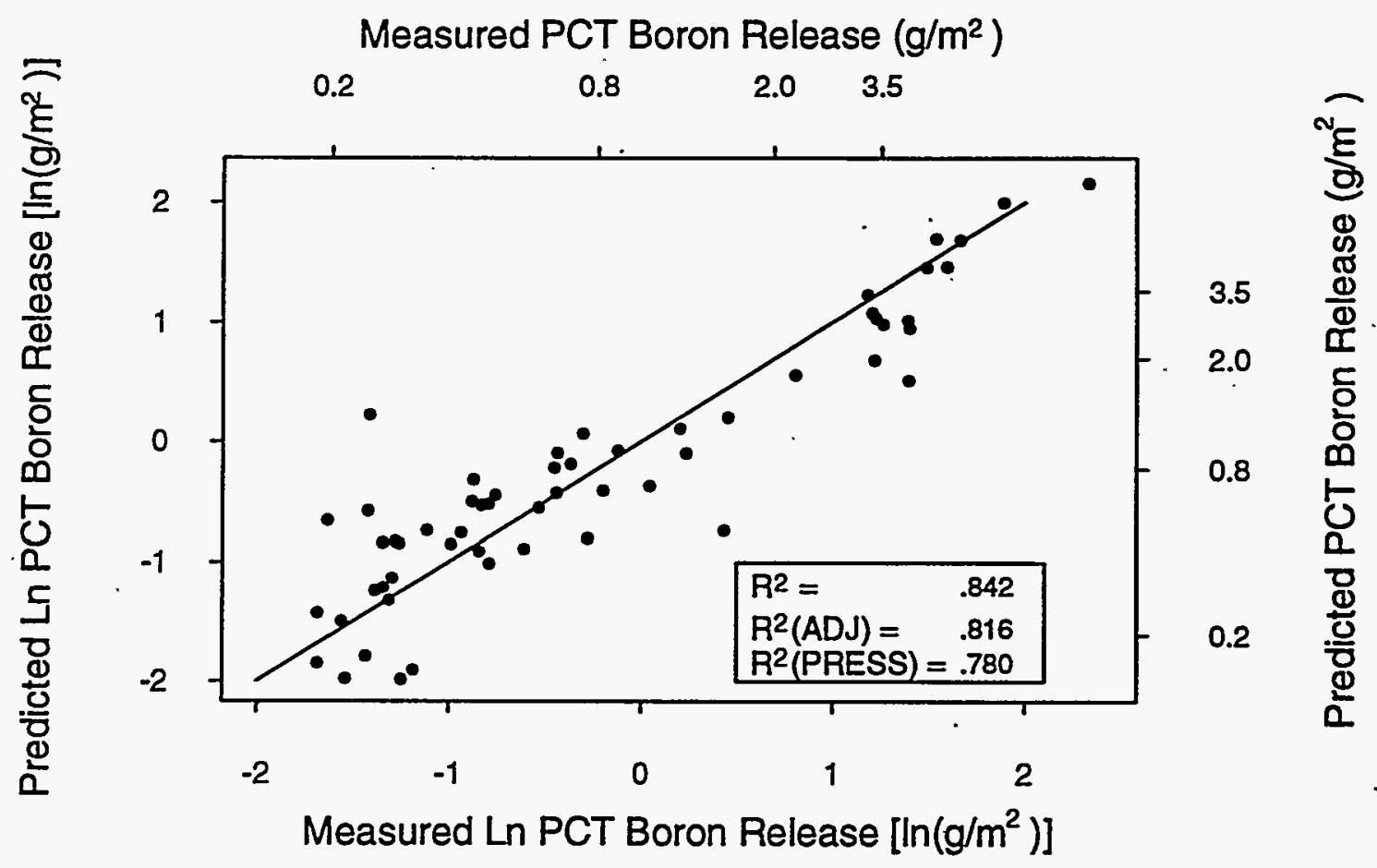

Figure C.20. Predicted Versus Measured Plot for the First-Order Mixture Model Fitted to the WVDP Data 
Measured PCT Boron Release $\left(\mathrm{g} / \mathrm{m}^{2}\right)$

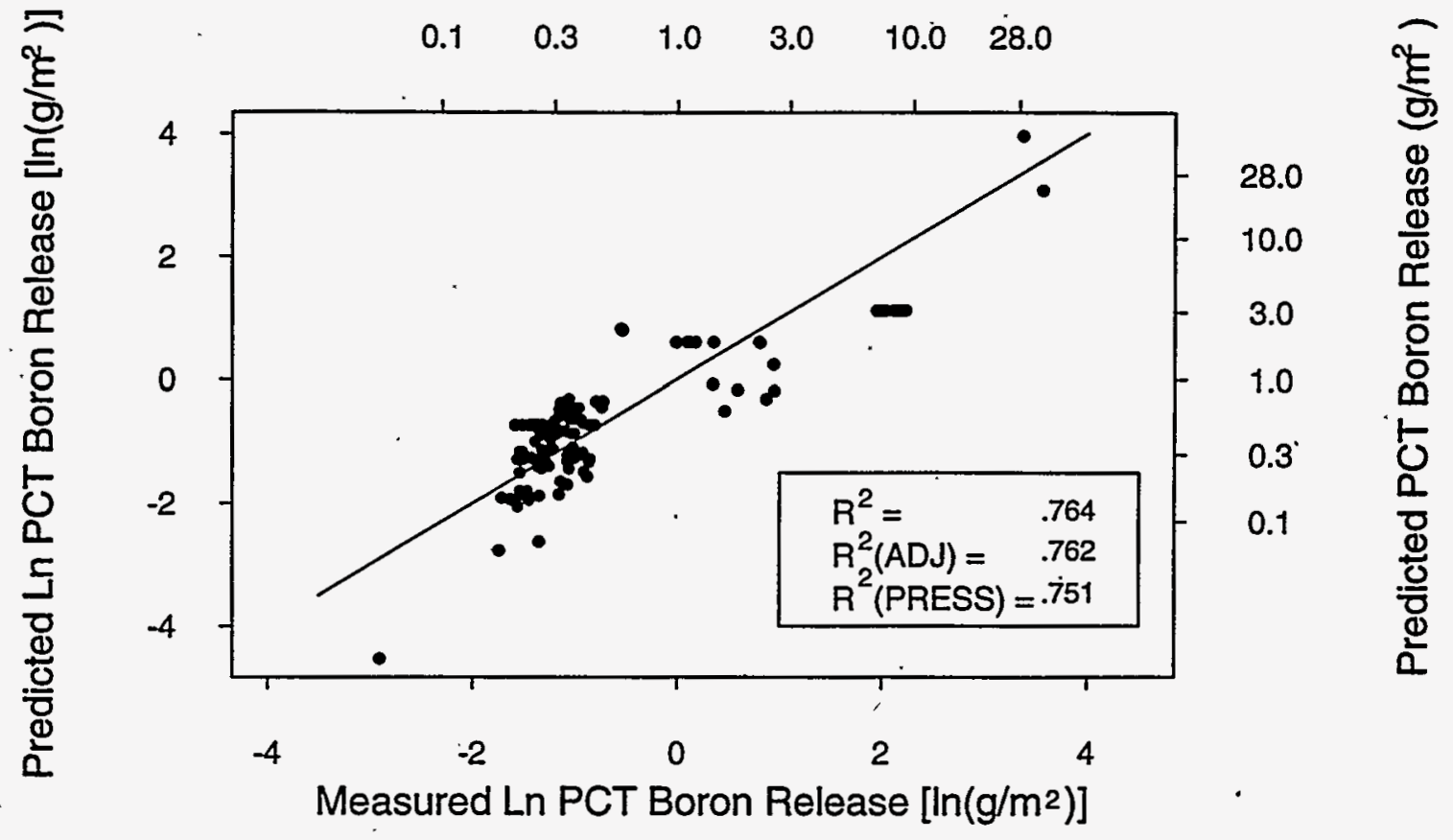

Figure C.21. Predicted Versus Measured Plot for the Free Energy of Hydration Model Fitted to the SRTC Homogeneous Data (All 137 Observations)

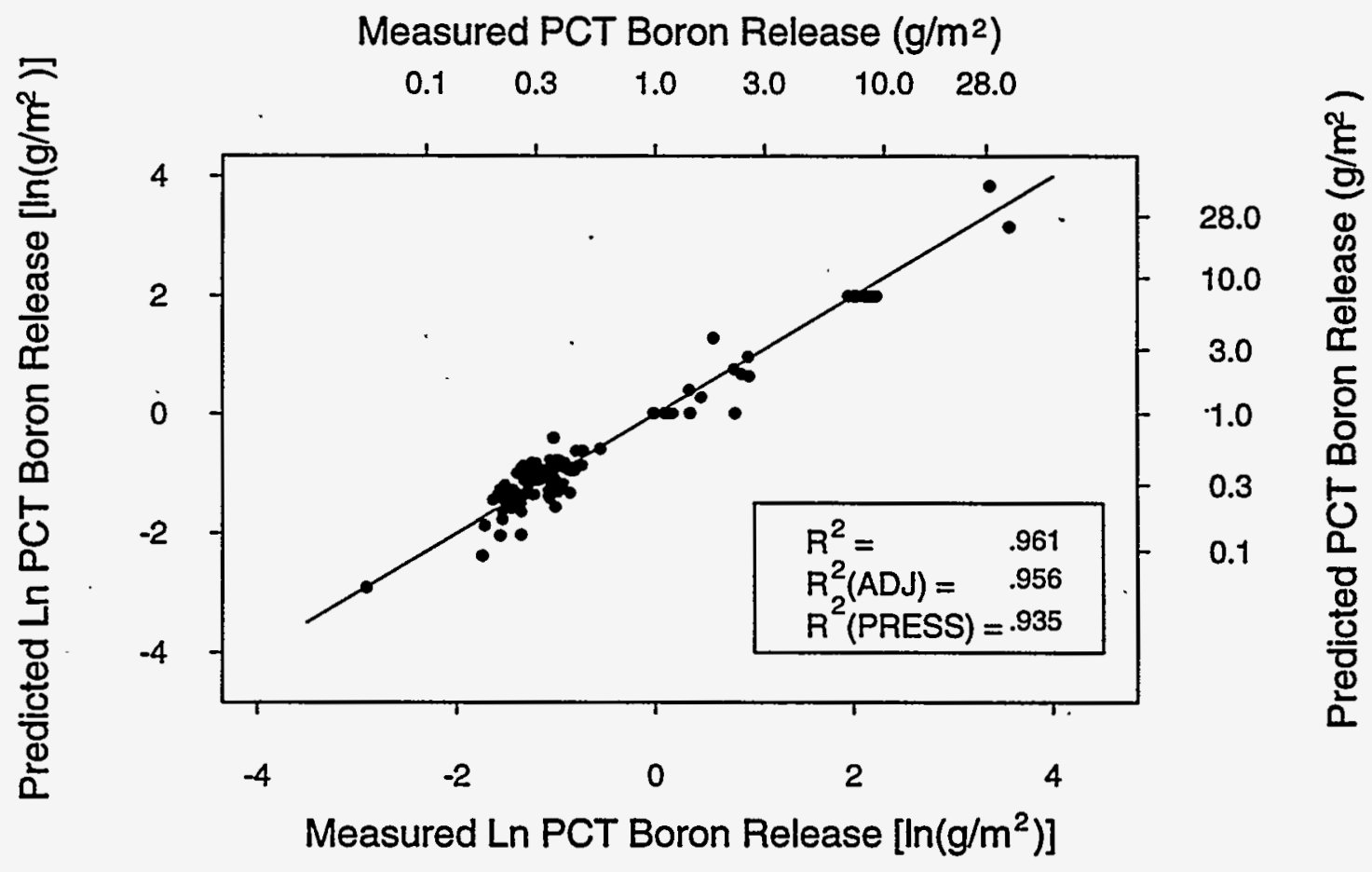

Figure C.22. Predicted Versus Measured Plot for the First-Order Mixture Model Fitted to the SRTC Homogeneous Data (All 137 Observations) 
Measured PCT Boron Release ( $\left.\mathrm{g} / \mathrm{m}^{2}\right)$

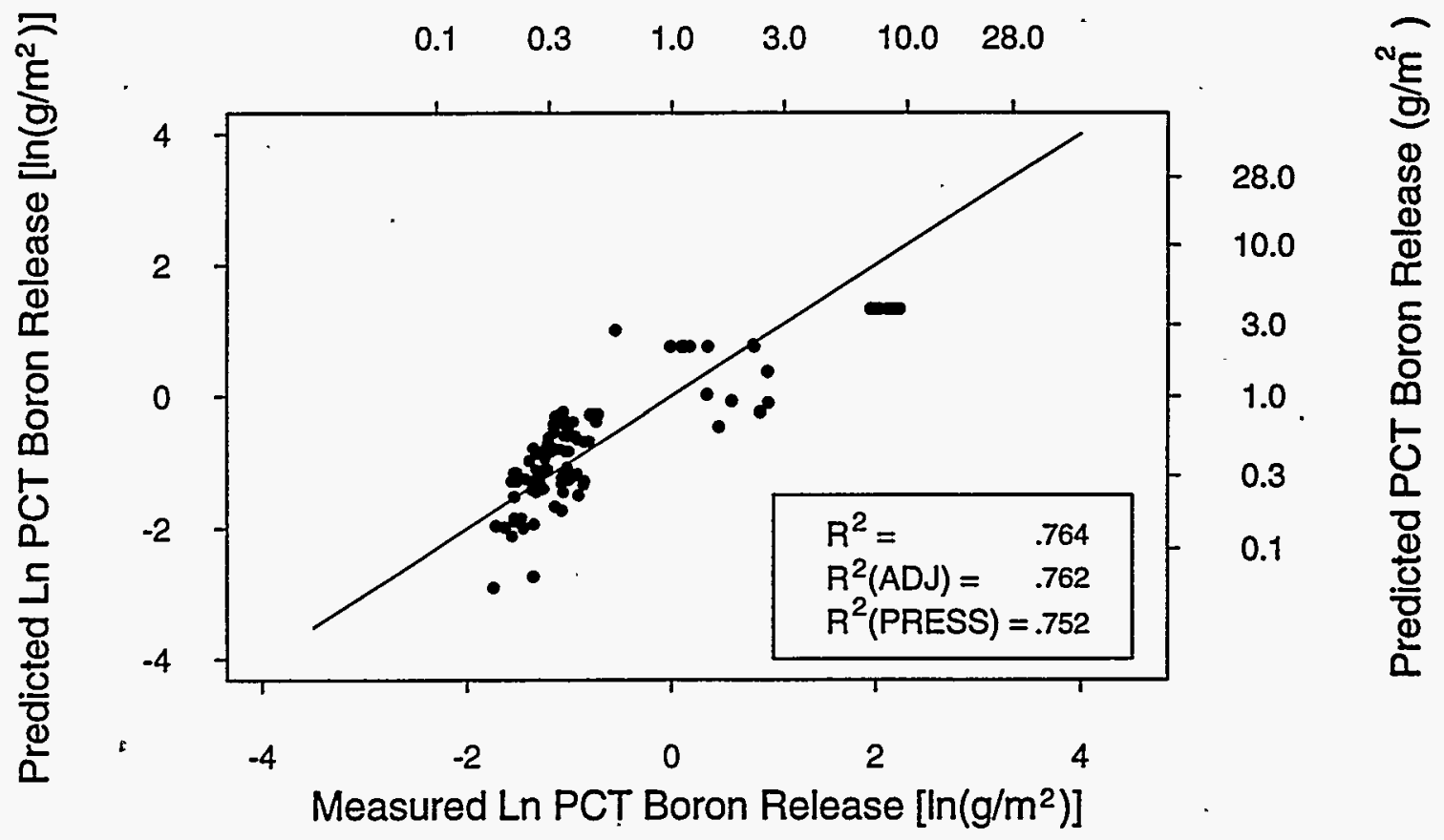

Figure C.23. Predicted Versus Measured Plot for the Free Energy of Hydration Model Fitted to the SRTC Homogeneous Data (118 Observations)

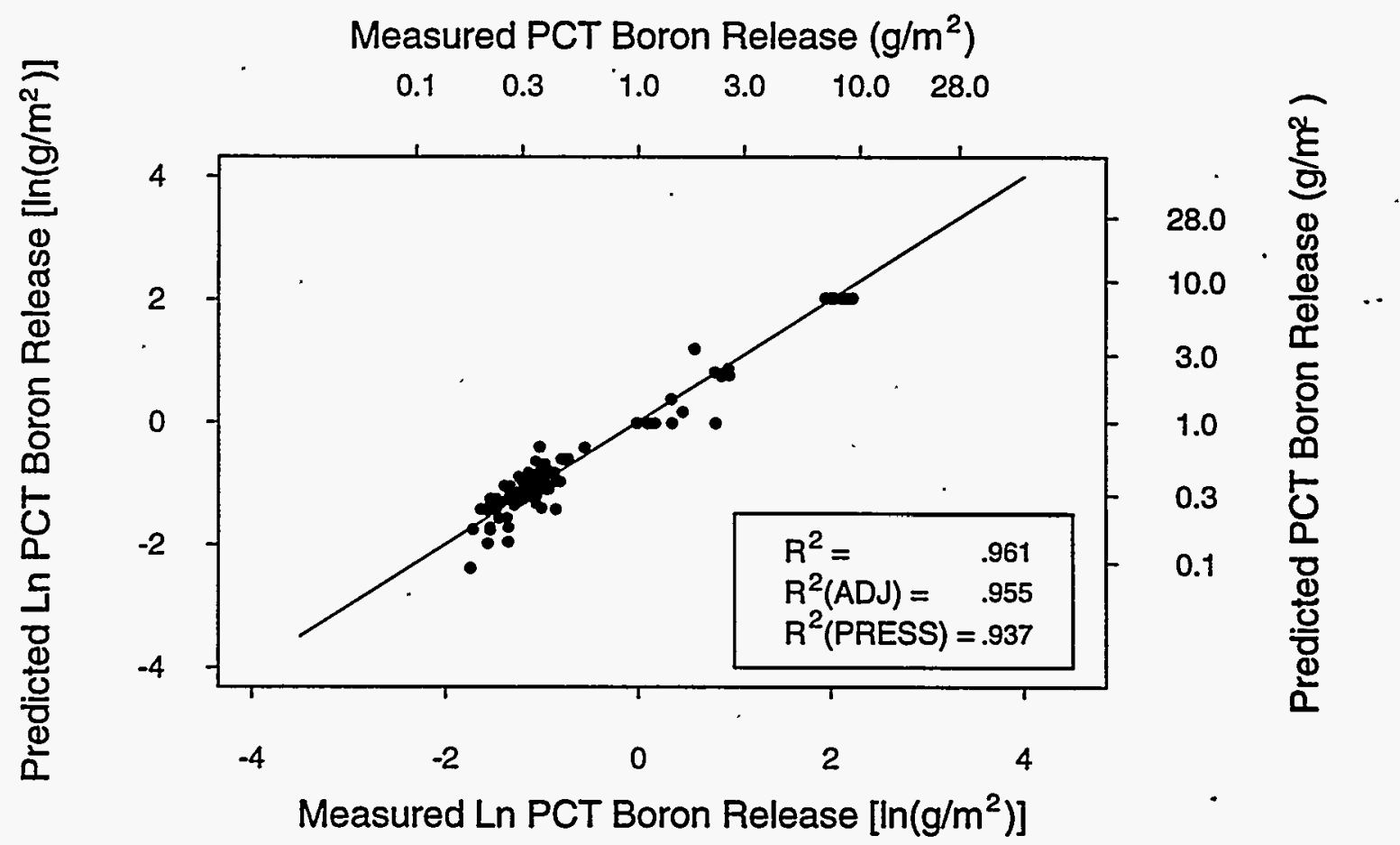

Figure C.24. Predicted Versus Measured Plot for the First-Order Mixture Model Fitted to the SRTC Homogeneous Data (118 Observations) 
Measured PCT Boron Release $\left(\mathrm{g} / \mathrm{m}^{2}\right)$

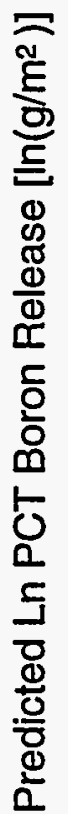

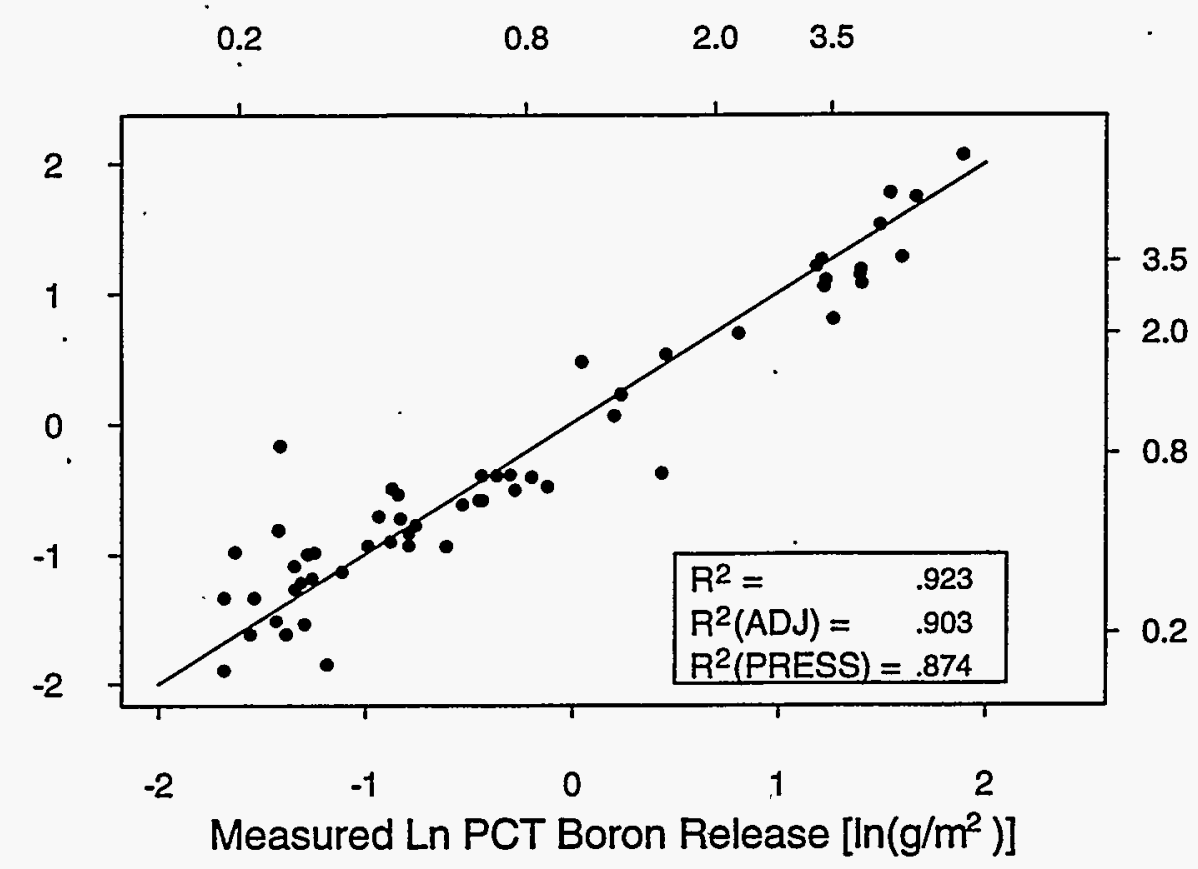

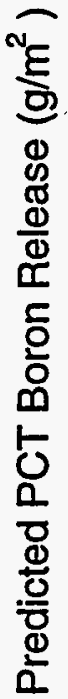

Figure C.25. Predicted Versus Measured Plot for the Reduced Second-Order Mixture Model Fitted to the WVDP Data

Measured PCT Boron Release $\left(\mathrm{g} / \mathrm{m}^{2}\right)$

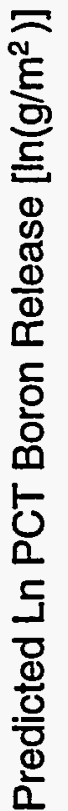

$$
\begin{array}{llllll}
0.1 & 0.3 & 1.0 & 3.0 & 10.0 & 28.0
\end{array}
$$

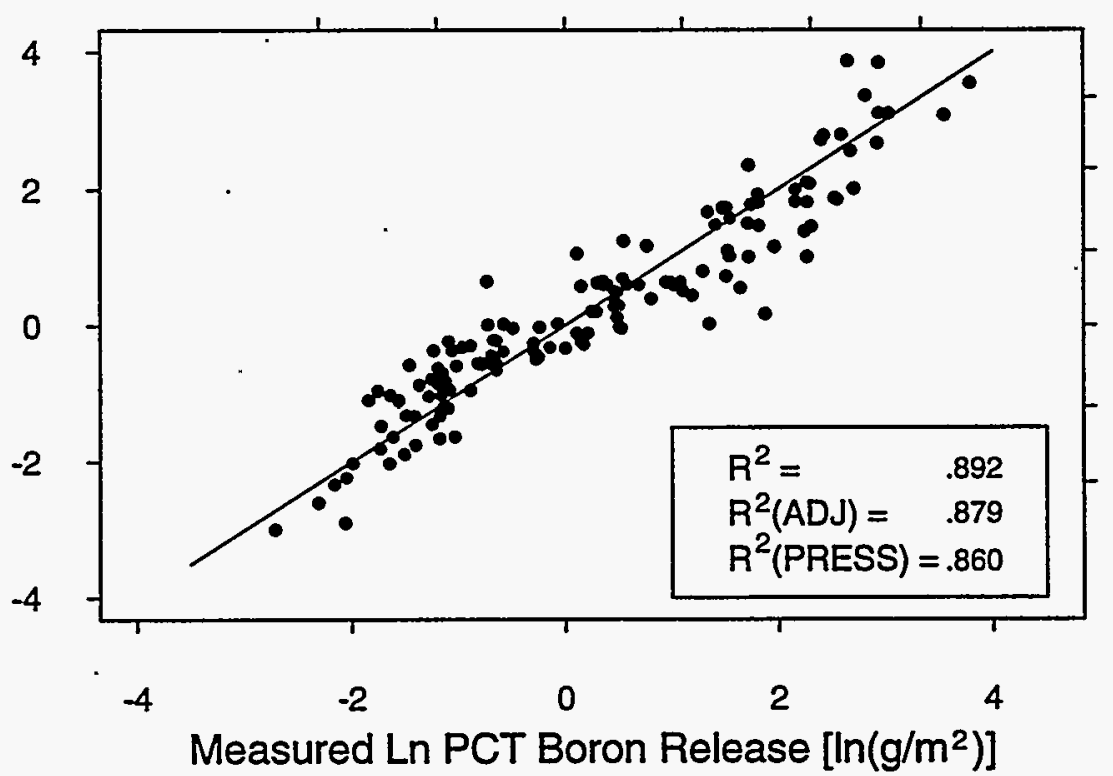

28.0

10.0

3.0

1.0

0.3

"ह

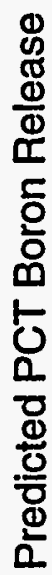

Figure C.26. Predicted Versus Measured Plot for the Reduced Second-Order Mixture Model Fitted to the CVS(all) Data 
Measured PCT Boron Release $\left(\mathrm{g} / \mathrm{m}^{2}\right)$

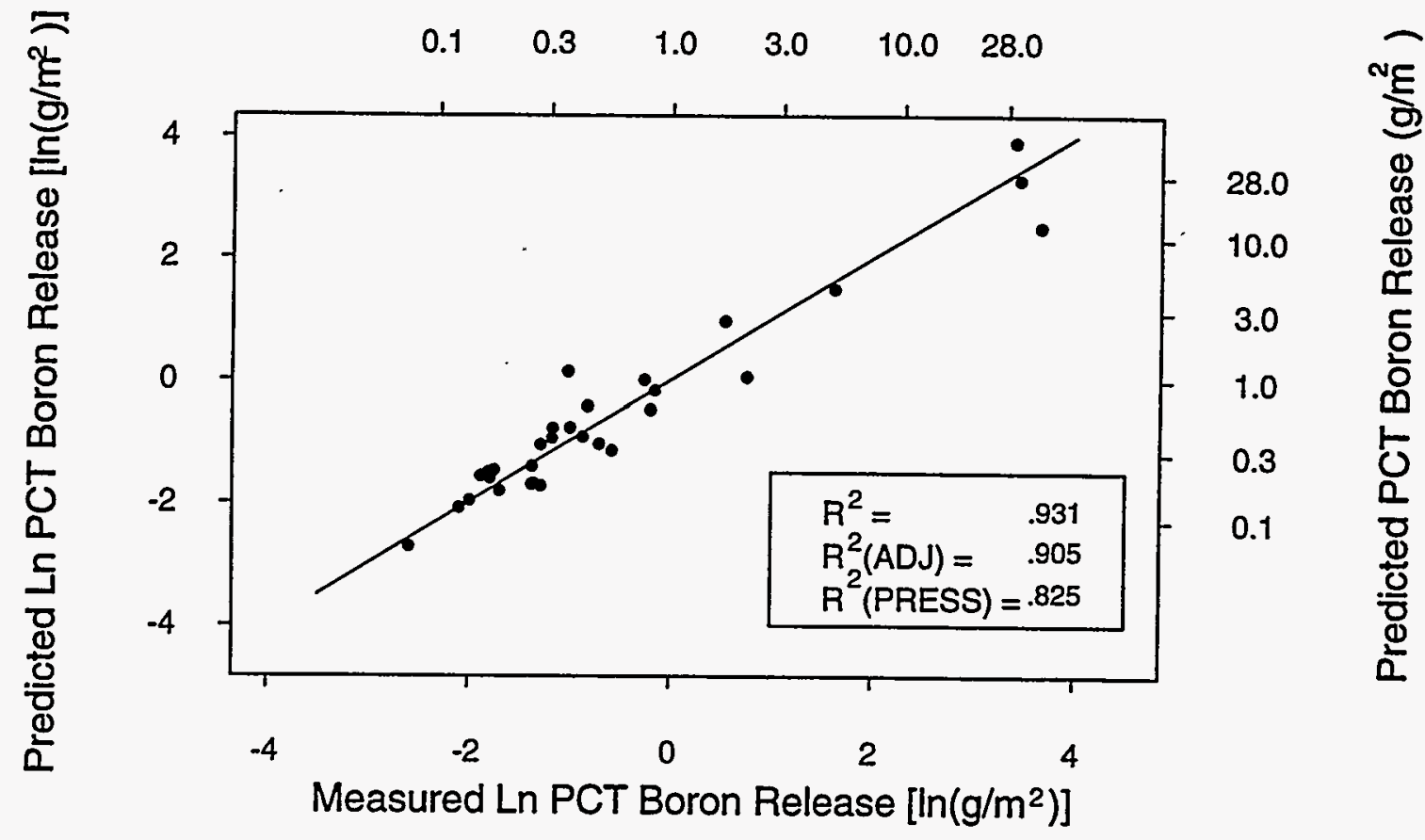

Figure C.27. Predicted Versus Measured Plot for the Reduced Second-Order Mixture Model Fitted to the Ramsey Data 



\section{DISTRIBUTION}

No. of

No. of

Copies

Copies

\section{OFFSITE}

1 W. L. Bourcier

Lawrence Livermore National Laboratory

1 T. Advocat

CEA-Cevalrito Marcovle

B.P. 171 .

30207 Bagnols-sur-Cèze Cedex

FRANCE

1 . S. M. Barnes

West Valley Nuclear Services

P. O. Box 191

West Valley, NY 14171

1 J. K. Bates

Argonne National Laboratory

$9700 \mathrm{~S}$. Cass Avenue

Argonne, IL 60439-4837

1 S. O. Bates

Lockheed Idaho Technology Co.

812 Dickson

Idaho Falls, ID 83402

1 T. H. Bertell

Science Applications Int'l Corp.

20300 Century Blvd.

Suite 200B

Germantown, MD 20874

1 N. E. Bibler

Westinghouse Savannah River Co.

P.O. Box 616

Aiken, SC 29802

1 D. F. Bickford

Westinghouse Savannah River Co.

P.O. Box 616

Aiken, SC 29802

1 L. H. Cadoff

West Valley Nuclear Services

P. O. Box 191

West Valley, NY 14171

1 K. A. Chacey, EM-361

DOE Office of Environmental

Management

19901 Germantown Road

Germantown, MD 20585

1 D. Clark

University of Florida

Advanced Materials Rèsearch

One Progress Blvd. \#14

Alachua, FL 32615

1 I. A. Colemen, EM-35

DOE Office of Environmental

Management

19901 Germantown Road

Germantown, MD 20585

1 R. R. Borisch

West Valley Nuclear Services

P. O. Box 191

West Valley, NY 14171

C. R. Cooley, EM-55

DOE Office of Environmental

Management

19901 Germantown Road

Germantown, MD 20585

Distr.1 
No. of

Copies

A. R. Cooper

Case. Western Reserve Univ.

Dept. Materials Science \& Eng.

10900 Euclid Avenue

Cleveland, $\mathrm{OH} 44106$

1 S. Cowan, EM-30

DOE Office of Environmental

Management

19901 Germantown Road

Germantown, MD 20585

1 D. E. Day

University of Missouri-Rolla

Materials Research Center

Rolla, MO . 65401

1 I. J. Dempster

West Valley Nuclear Services

P. O. Box 191

West Valley, NY 14171

1 R. H. Doremus

Rensselaer Polytechnic Inst.

Materials Engineering Dept.

Troy, NY 12180-3950

1 D. Dreyfus; RW-1.

DOE Office of Civilian

Radioactive Waste Management

1000 Independence Ave. S.W.

Wạshington, D.C. 20585

1 A. Drobot

West Valley Nuclear Services

P. O. Box 191

West Valley, NY 14171

1 J. P. Duckworth

Operations \& Process Appraisals, Inc.

2604 Glenwood Circle

Idaho Falls, ID 83404

1 W. Ebert

Argonne National Laboratory

9700 S. Cass Avenue

Argonne, II 60439-4837
No. of

Copies

1

T. B. Edwards

Westinghouse Savannah River Co.

P.O. Box 616

Aiken, SC 29802

1 A. E. G. Ellison

Argonne National Laboratory

9700 S. Cass Avenue

Argonne, IL 60439-4837

1 R. E. Ewing

University of New Mexico

Albuquerque, NM 87131

1 J. J. Fiore, EM-423

DOE Office of Environmental

Management

19901 Germantown Road

Germantown, MD 20585

1 C. W. Forsberg

Oak Ridge National Laboratory

P.O. Box 2008

105 Mitchell Rd.

Oak Ridge, TN 37831-6495

1 C. Frank, EM-50

DOE Office of Environmental

Management

19901 Germantown Road

Germantown, MD 20585

1 M. Frei, EM-34

DOE Office of Environmental

Management

19901 Germantown Road

Germantown, MD 20585

1 L. B. Gannon

Science Applications Int'l Corp. 20201 Century Blvd., 3rd Floor Germantown, MD 20874

1 T. M. Gilliam

Oak Ridge National Laboratory

P.O. Box 2008

M.S. 6202

Oak Ridge, TN 37831-6202 
No. of

Copies

1 T.C. Gunter

U.S. Department of Energy

Savannah River Operations Office

P.O. Box A

Aiken, S.C. 29802

1 L. L. Hench

University of Florida

Advanced Materials Research

One Progress Blvd. \#14

Alachua, FL 32615

1 J. Hennessey, EM-361

DOE Office of Environmental

Management

19901 Germantown Road

Germantown, MD 20585

1 E. W. Holtzscheiter

Westinghouse Savannah River Co.

P.O. Box 616

Aiken, SC 29802

1 V. J. Jain

West Valley Nuclear Services

P. O. Box 191

West Valley, NY 14171

1 R. F. Jakubik

BDM International, Inc. 20300 Century Blvd.

Germantown, MD 20874

1

C. M. Jantzen

Westinghouse Savannah River Co.

P.O. Box 616

Aiken, SC 29802

1 D. E. Karl

West Valley Nuclear Services

P. O. Box 191

West Valley, NY 14171

1 W. S. Ketola

DOE West Valley Project

P.O. Box 191

West Valley, NY 14171
No. of

Copies

1 R. B. King

Regents Professor of Chemistry

University of Georgia

1001 Cedar Street

Athens, GA 30602

1 J. R. Knight

Westinghouse Savannah River Co.

P.O. Box 616

Aiken, SC 29802

1 W. LaCourse

Alfred University

NYS College of Ceramics

Alfred, NY 14802

1 A. Loida

Institut fur Nukleare Entsorgungstechnik

Forschungszentrum Karlsruhe GmbH

Postfach 3640

D-76021 Karlsruhe 1

GERMANY

$1 \quad$ R. E. Lowder

Mac Tec

1309 E. St. Louis Ave., \#3

Las Vegas, NV 89104

1 W. Lutze

Center for Radioactive Waste Management

209 Farris Engineering Center

U. of New Mexico

Albuquerque, NM 87131

1 J. E. Lytle, EM-30

DOE Office of Environmental

Management

19901 Germantown Road

Germantown, MD 20585

1 P. Macedo

Vitreous State Laboratory

Catholic University of America .

Washington, D.C. 20064

1 J. L. Mahoney

West Valley Nuclear Services

P. O. Box 191

West Valley, NY 14171 
No. of

Copies

$54 \quad$ Pacific Northwest National Laboratory

G. H. Beeman

P7-75

W. F. Bonner

K9-14

T. M. Brouns

K9-08

B. C. Bunker

$\mathrm{K} 2-45$

J. M. Creer

K9-80

M. L. Elliott

P7-41

X. D. Feng

P8-44

J. H. Holbrook

K9-73

L. K. Holton

P7-43

P. R. Hrma

P8-37

P. K. Kearns

K6-51

K. D. Keefer

$\mathrm{K} 2-25$

H. $\mathrm{Li}$

P8-44

N. J. Lombardo

K9-69

B. P. McGrail

$\mathrm{K} 2-44$

G. B. Mellinger

K6-51

E. V. Morrey

P7-19

J. M. Perez, Jr.

P7-41

G. F. Piepel (10)

K5-12

R. K. Quinn

K9-69

P. E. Redgate (5)

K5-12

W. G. Richmond

K6-51

R. J. Robertus

K3-75

F. M. Ryan

K5-12

M. J. Schweiger

P8-37

S. C. Slate

K9-14

T. L. Stewart

K9-91

J. D. Vienna (5)

P8-44

J. H. Westsik, Jr.

K9-80

K. D. Wiemers

$\mathrm{K} 6-51$

Information Release

Office (7)

K1-11 